University of Tennessee Health Science Center

UTHSC Digital Commons

$12-2014$

\title{
Computer-Aided Drug Design and Discovery, Screening and Synthesis of Small Molecule Inhibitors of Nucleoside Transporters
}

Hilaire Colleen Playa

University of Tennessee Health Science Center

Follow this and additional works at: https://dc.uthsc.edu/dissertations

Part of the Medicinal and Pharmaceutical Chemistry Commons, and the Pharmaceutics and Drug Design Commons

\section{Recommended Citation}

Playa, Hilaire Colleen , "Computer-Aided Drug Design and Discovery, Screening and Synthesis of Small Molecule Inhibitors of Nucleoside Transporters" (2014). Theses and Dissertations (ETD). Paper 398. http://dx.doi.org/10.21007/etd.cghs.2014.0247. 


\title{
Computer-Aided Drug Design and Discovery, Screening and Synthesis of Small Molecule Inhibitors of Nucleoside Transporters
}

\author{
Abstract \\ Using prior biological data, pharmacophore models were made for hCNT1, hCNT3, hENT1, and hENT4. \\ The hCNT3 and hCNT1 pharmacophore were used to select compounds for biological testing. The \\ NBMPR analogue and dipyridamole analogue hENT1 pharmacophores were compared to each other and \\ to a combined pharmacophore for hENT1. The dipyridamole analogue pharmacophore better predicted \\ non-nucleoside small molecule inhibitors, and as such appears to be the better tool for aiding in the \\ design of new small molecule inhibitors. The hCNT3 pharmacophore failed to select active compounds \\ and as such must be redesigned. The hCNT1 pharmacophore succeeded in identifying two moderately \\ active compounds and when the hits were added into the data set to make a new pharmacophore, a more \\ statistically valid model was achieved. The hCNT1 model is a statistically valid model for in silico \\ screening of compounds. \\ Document Type \\ Dissertation \\ Degree Name \\ Doctor of Philosophy (PhD) \\ Program \\ Pharmaceutical Sciences \\ Research Advisor \\ John K. Buolamwini, Ph.D. \\ Keywords \\ SLC29, nucleoside transporters, nucleosides, PMAT, QSAR, SLC28 \\ Subject Categories \\ Medicinal and Pharmaceutical Chemistry | Medicine and Health Sciences | Pharmaceutics and Drug \\ Design | Pharmacy and Pharmaceutical Sciences
}




\title{
Computer-Aided Drug Design and Discovery, Screening and Synthesis of Small Molecule Inhibitors of Nucleoside Transporters
}

\author{
A Dissertation \\ Presented for \\ The Graduate Studies Council \\ The University of Tennessee \\ Health Science Center
}

\author{
In Partial Fulfillment \\ Of the Requirements for the Degree \\ Doctor of Philosophy \\ From The University of Tennessee
}

By

Hilaire Colleen Playa

December 2014 
Copyright (C) 2014 by Hilaire Colleen Playa. All rights reserved. 


\section{DEDICATION}

I dedicate this dissertation to every teacher who inspired, encouraged, and pushed me to do my best and to my friends and family, especially my grandparents who provided crucial support on my journey to this degree. 


\section{ACKNOWLEDGEMENTS}

As is usual for research, none of this could have been accomplished without the mentorship, guidance, and help of many people. I thank my advisor, Dr. John Buolamwini for the opportunity to work in his lab and for his support. I thank my committee members, Dr. Peter Bridson, Dr. Isaac Donkor, Dr. Duane Miller, and Dr. Bob Moore for their advice, support, and also for their criticism, as that is what helps us learn. In addition, I'd like to acknowledge my gratitude to the individuals I have worked with the past five years: Dr. Chunmei Wang, Shan Sun, Dr. Hemantkumar Deokar, Mrunalini Narkar-Deokar, and Horrick Sharma. I'd also like to express my appreciation to allthe faculty, staff, and students not named herein that have helped me through my research endeavors. Lastly, I'd like to thank the University of Tennessee for the academic opportunity and its generous financial support. 


\begin{abstract}
Using prior biological data, pharmacophore models were made for hCNT1, hCNT3, hENT1, and hENT4. The hCNT3 and hCNT1 pharmacophore were used to select compounds for biological testing. The NBMPR analogue and dipyridamole analogue hENT1 pharmacophores were compared to each other and to a combined pharmacophore for hENT1. The dipyridamole analogue pharmacophore better predicted non-nucleoside small molecule inhibitors, and as such appears to be the better tool for aiding in the design of new small molecule inhibitors. The hCNT3 pharmacophore failed to select active compounds and as such must be redesigned. The hCNT1 pharmacophore succeeded in identifying two moderately active compounds and when the hits were added into the data set to make a new pharmacophore, a more statistically valid model was achieved. The hCNT1 model is a statistically valid model for in silico screening of compounds.
\end{abstract}




\section{TABLE OF CONTENTS}

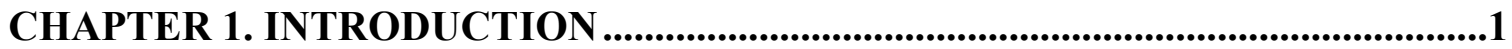

Classification of Nucleoside Transporters .............................................................. 1

Nucleoside Transporter Substrate Specificity.......................................................... 1

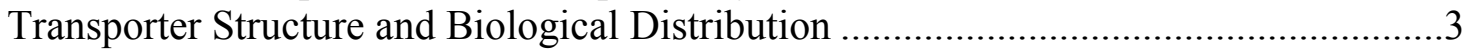

Nucleoside Transporters and Species Variations...................................................

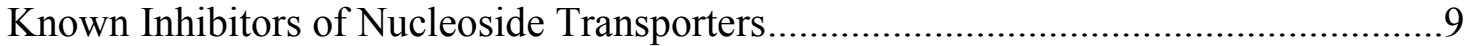

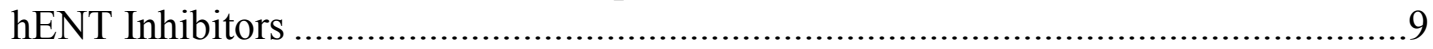

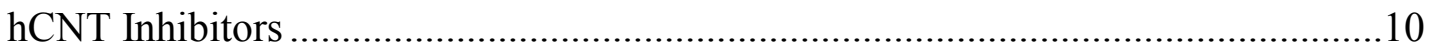

Nucleoside Transporter Inhibitor Applications and Purpose of Study ........................17

CHAPTER 2. COMPUTER MODELING STUDIES ............................................18

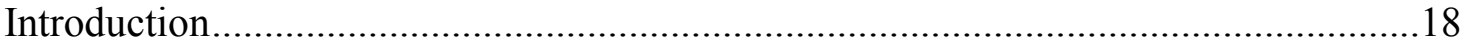

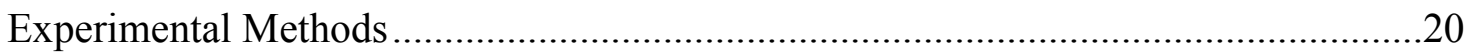

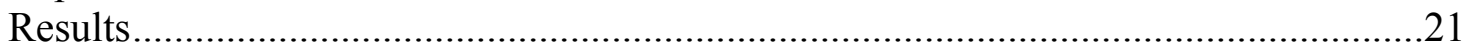

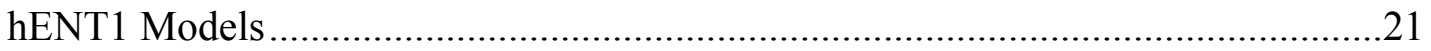

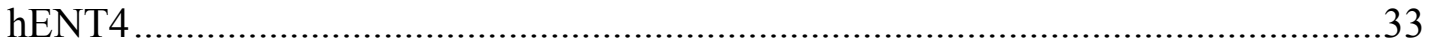

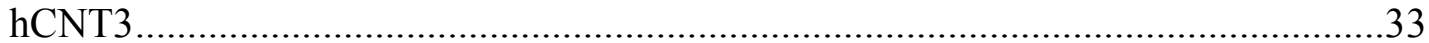

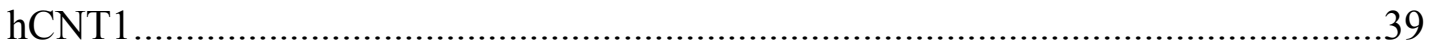

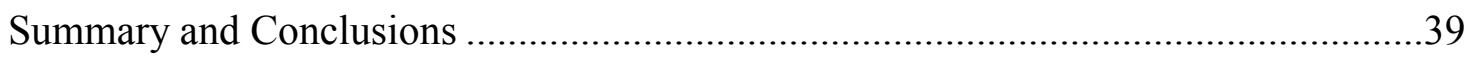

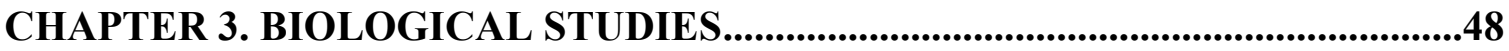

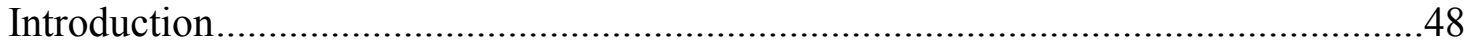

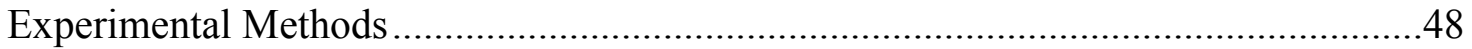

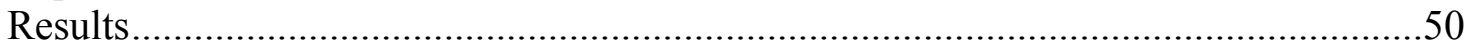

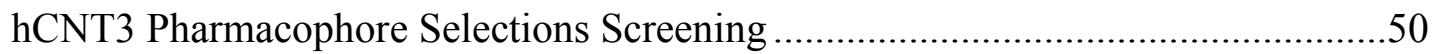

hCNT1 Pharmacophore Selections Screening ........................................................69

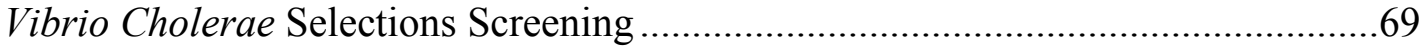

hCNT1 Homology Model Selections Screening.....................................................69

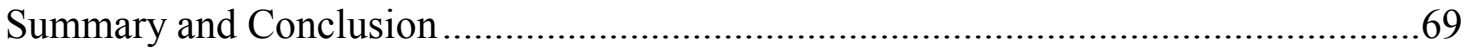

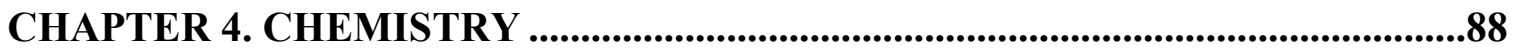

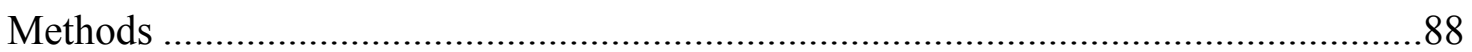

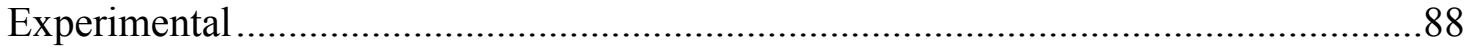

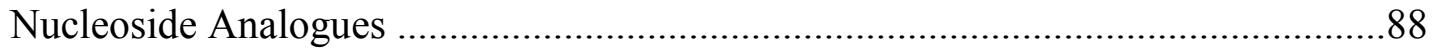

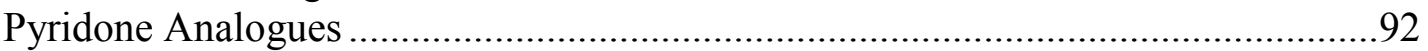

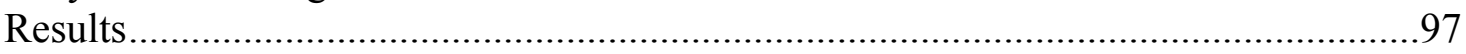

CHAPTER 5. BROAD INSTITUTE COLLABRATIVE STUDY ..........................101

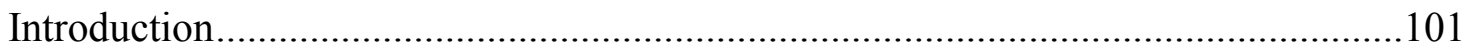

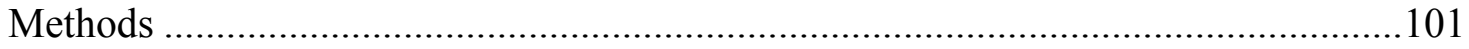

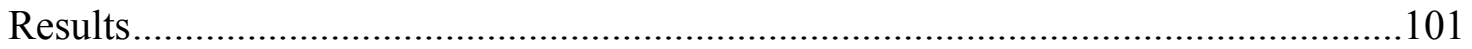


CHAPTER 6. CONCLUSION AND FUTURE STUDIES.............................................108

LIST OF REFERENCES ................................................................................................111

APPENDIX. SUPPLEMENTAL DATA.........................................................................115

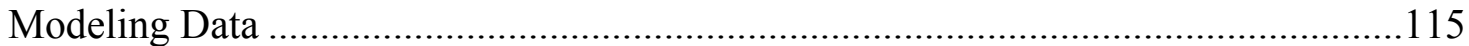

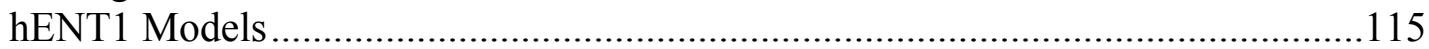

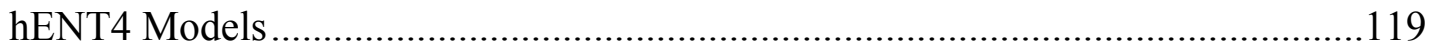

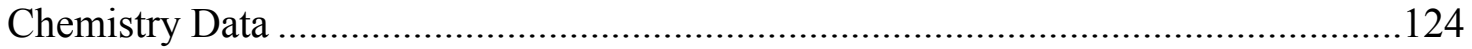

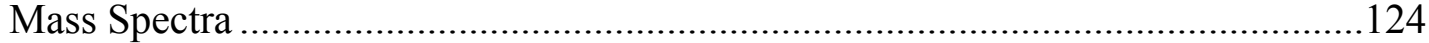

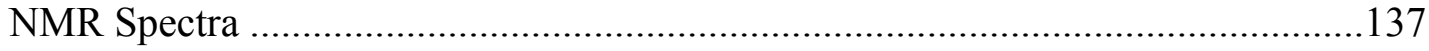

VITA 


\section{LIST OF TABLES}

Table 1-1. Nucleoside transporter tissue distribution in humans ...............................6

Table 1-2. Current known nucleoside transporter mutations ........................................8

Table 1-3. Survey of non-nucleoside inhibitors of ENT1 ......................................11

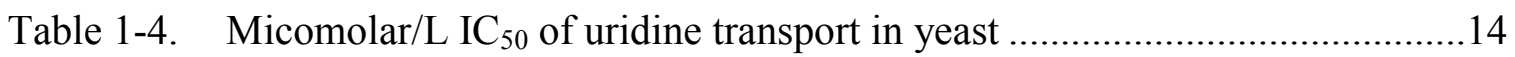

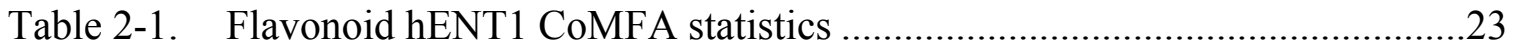

Table 2-2. Dipyridamole and NBMPR analogue pharmacophore aligned models ........28

Table 2-3. “Combined” pharmacophore CoMFA and CoMSIA PLS statistics .............32

Table 2-4. PLS statistics for hENT4 flavonoid pharmacophore-aligned models...........34

Table 2-5. PLS statistics for hENT4 dipyridamole pharmacophore-aligned models.....36

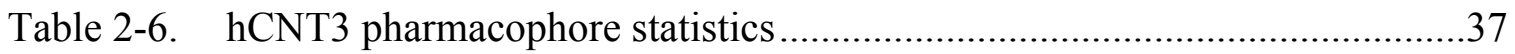

Table 2-7. hCNT1 flavone pharmacophore statistics for original and hit-enriched

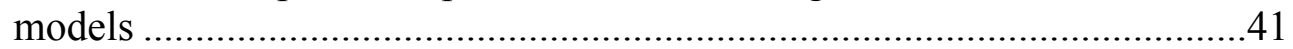

Table 2-8. Dipyridamole pharmacophore search predictions ...................................42

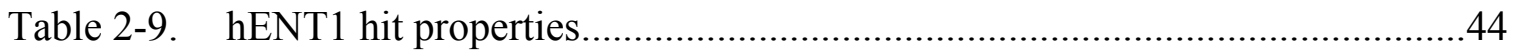

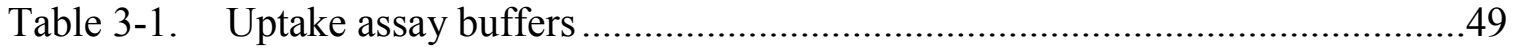

Table 3-2. Inhibitor controls used for uptake assays ...........................................51

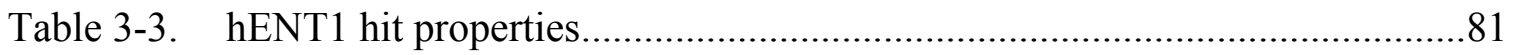

Table 4-1. Synthesized nucleoside derivative screening results .................................98

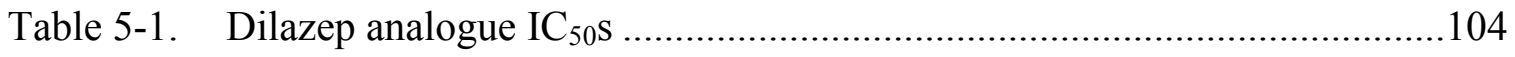




\section{LIST OF FIGURES}

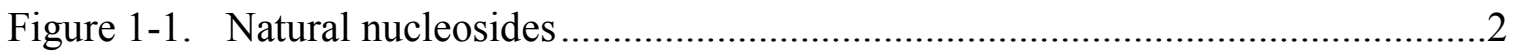

Figure 1-2. Classic nucleoside inhibitors..................................................................

Figure 1-3. Graphical depiction of human nucleoside transporter distributions ...............7

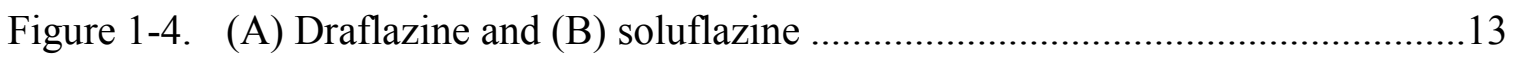

Figure 1-5. WL30 a potent and selective hENT4 inhibitor ............................................14

Figure 1-6. 8-position modified purine analogue ………………………………….......14

Figure 1-7. Chemotherapy agents with nucleoside transporter inhibitory activity .........15

Figure 1-8. (A) Phlorizin (B) phlorizin analogue …………………………………....... 16

Figure 2-1. Nucleoside numbering on (A) purine and (B) pyrimidine............................19

Figure 2-2. Flavonoid active and inactive sets used for the creation of ENT1

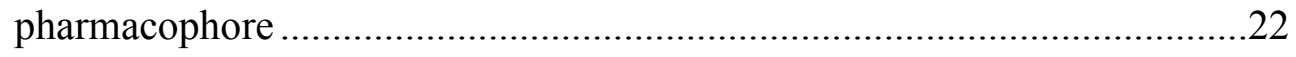

Figure 2-3. Flavonoid ENT1 5-point pharmacophore hypothesis ...................................22

Figure 2-4. Predicted versus actual - $\log \mathrm{IC}_{50}$ of flavonoid analogues ...........................23

Figure 2-5. Dipyridamole analogues used for the creation of hENT1 pharmacophore...25

Figure 2-6. Active nucleoside analogues used for hENT1 pharmacophore ….................25

Figure 2-7. Synthesized nucleoside analogues …………………................................26

Figure 2-8. Prediction plot of hENT1 NBMPR CoMFA model ......................................26

Figure 2-9. Prediction plot of hENT1 NBMPR CoMSIA model .....................................27

Figure 2-10. NBMPR (A) pharmacophore and dipyridamole (B) pharmacophore...........27

Figure 2-11. Active set for ENT1 "combined" pharmacophore..........................................29

Figure 2-12. Inactive set for hENT1 "combined" pharmacophore......................................30

Figure 2-13. "Combined" pharmacophore hypothesis with active compound 13A

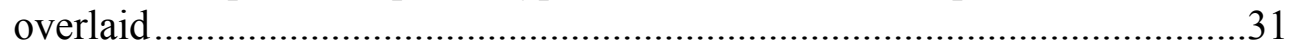

Figure 2-14. hENT4 flavonoid compounds used for pharmacophore design......................35 
Figure 2-15. Residuals plot of CoMFA dipyridamole analogues..................................37

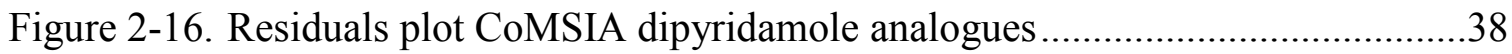

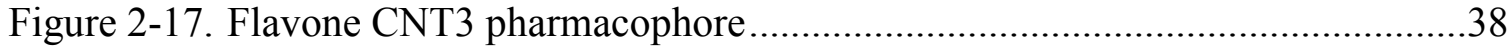

Figure 2-18. Flavones used for hCNT3 pharmacophore design..................................40

Figure 2-19. hCNT1 flavone pharmacophore ........................................................41

Figure 2-20. Hit enriched hCNT1 pharmacophore...................................................42

Figure $3-1$. Uptake assay procedure workflow ......................................................52

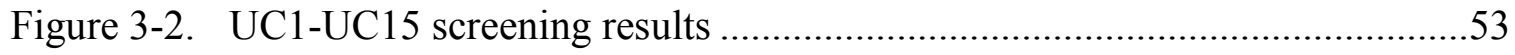

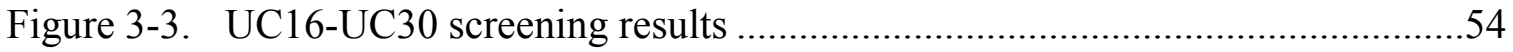

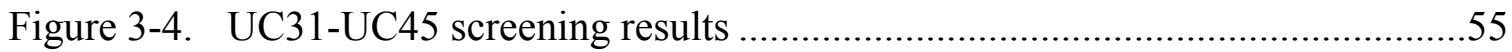

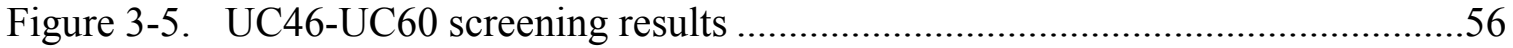

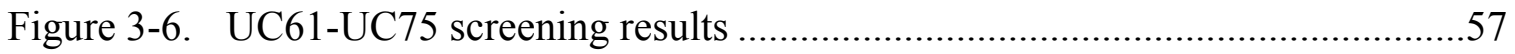

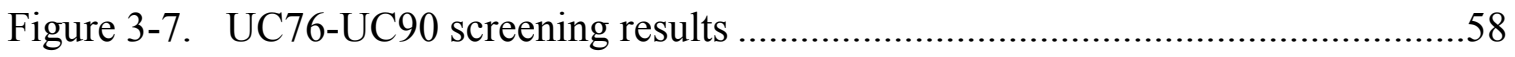

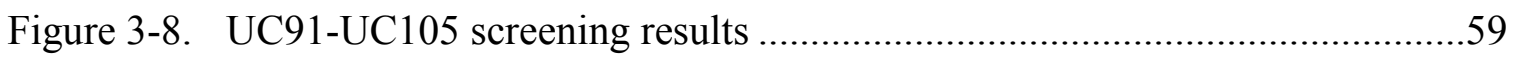

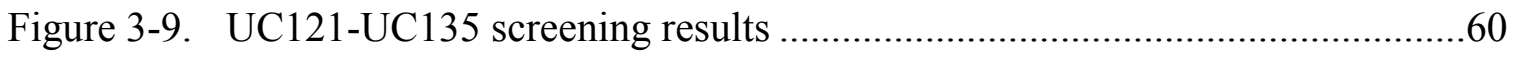

Figure $3-10$. UC136-UC150 screening results .....................................................61

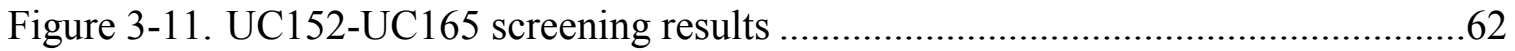

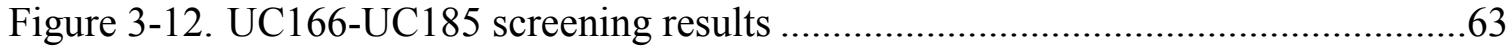

Figure $3-13$. UC186-UC192 screening results ......................................................64

Figure $3-14$. Cross screen of rENT2 hits against hENT2 …....................................65

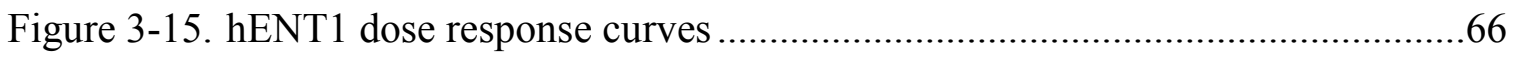

Figure $3-16$. hENT4 dose response curves ...........................................................68

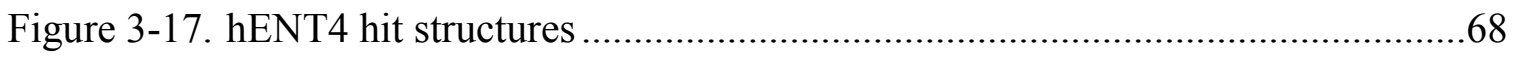

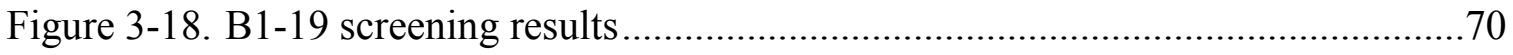

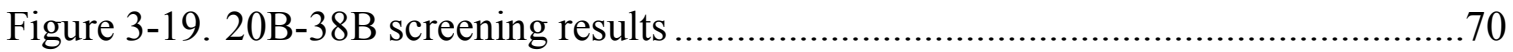




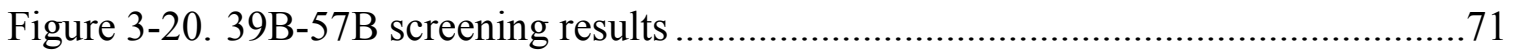

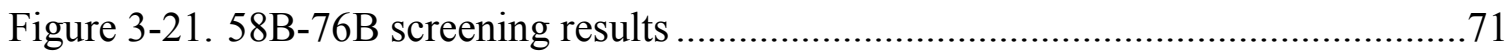

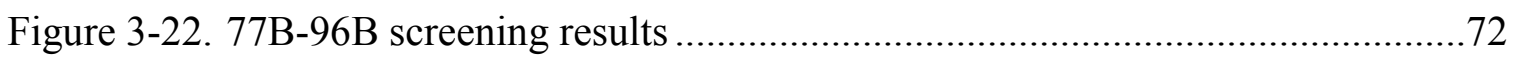

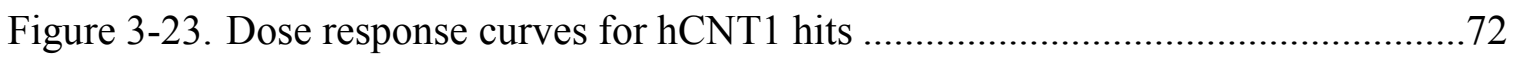

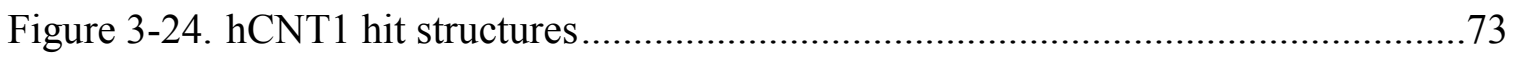

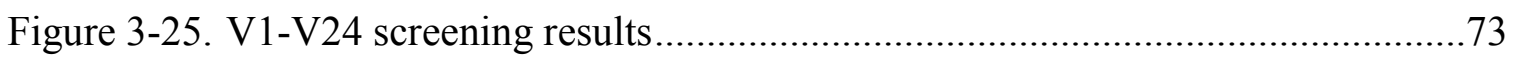

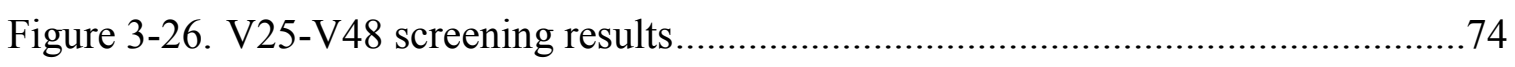

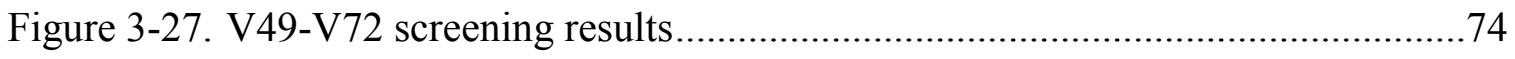

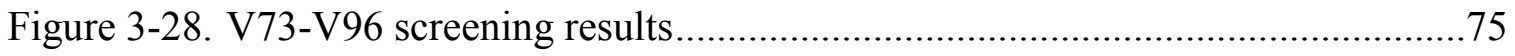

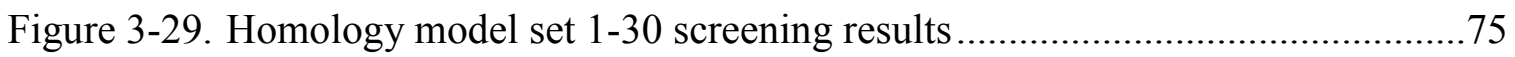

Figure 3-30. Homology model set 31-60 screening results ....................................... 76

Figure 3-31. Homology model set $61-90$ screening results ........................................ 76

Figure 3-32. Homology model set 91-120 screening results ......................................77

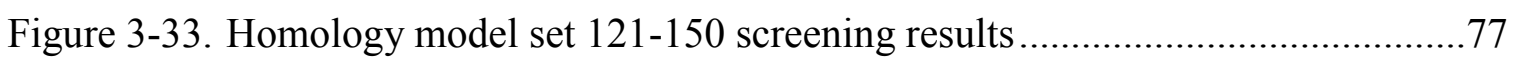

Figure 3-34. Homology model set 151-177 screening results ....................................78

Figure $3-35$. hCNT1 pharmacophore model screening results ....................................85

Figure $3-36$. Homology model compounds screening results ....................................86

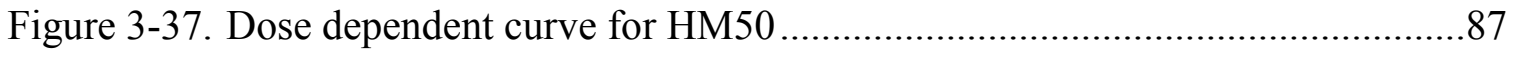

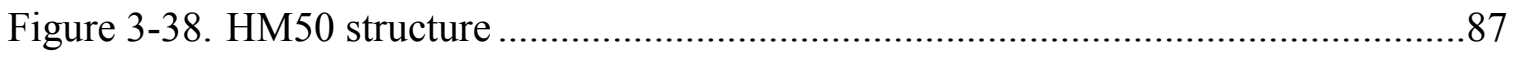

Figure 4-1. Synthesized and tested nucleoside analogues .......................................93

Figure 4-2. Synthesized and untested nucleoside analogues ....................................94

Figure 4-3. Synthesized and tested pyridones ......................................................94

Figure 4-4. Compound-4 structure …........................................................... 98

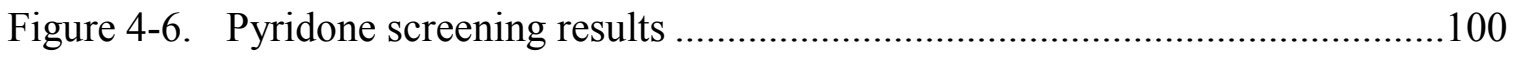

Figure 5-1. $10 \mu \mathrm{M}$ screening of A1-E11 against hENT1 and rENT2 .........................102 
Figure 5-2. $10 \mu \mathrm{M}$ screening of E13-I23 against hENT1 and rENT2 …......................102

Figure 5-3. $10 \mu \mathrm{M}$ screening of K1-O7 against hENT1 and rENT2 …........................103

Figure 5-4. Uptake inhibition comparison of rat and human ENT2 …………….........105

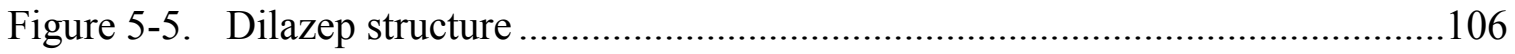

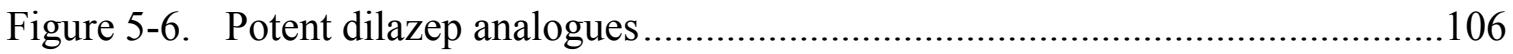

Figure 6-1. Dilazep and UC21 comparison ...........................................................110

Figure A-1. Flavonoid CoMFA contours of hENT1 ……….....................................115

Figure A-2. hENT1 dipyridamole CoMFA contours .................................................115

Figure A-3. hENT1 dipyridamole CoMSIA electrostatic and steric contours ................116

Figure A-4. hENT1 dipyridamole CoMSIA hydrophobic contours ................................116

Figure A-5. hENT1 dipyridamole CoMSIA hydrogen bond donor and acceptor contours

Figure A-6. NBMPR CoMFA hENT1 contours..........................................................117

Figure A-7. NBMPR hENT1 CoMSIA steric and electrostatic contours........................118

Figure A-8. NBMPR hENT1 CoMSIA hydrophobic contours .......................................118

Figure A-9. NBMPR hENT1 CoMSIA hydrogen bond donor and acceptor contours...119

Figure A-10.Dipyridamole hENT4 CoMFA contours ..................................................119

Figure A-11.Dipyridamole hENT4 CoMSIA steric and electrostatic contours ..............120

Figure A-12.Dipyridamole hENT4 CoMSIA hydrophobic contours ...............................120

Figure A-13. Dipyridamole hENT4 CoMSIA hydrogen bond donor and acceptor

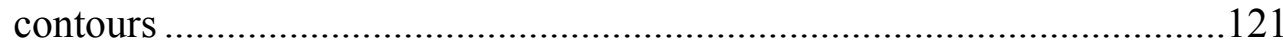

Figure A-14.Flavone hENT4 CoMFA contours ...........................................................121

Figure A-15. Flavone hENT4 CoMSIA steric and electrostatic contours........................122

Figure A-16. Flavone hENT4 CoMSIA hydrophobic contours .......................................122

Figure A-17.Flavone hENT4 CoMSIA hydrogen bond donor and acceptor contours....123 


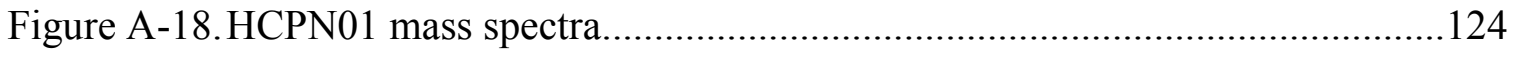

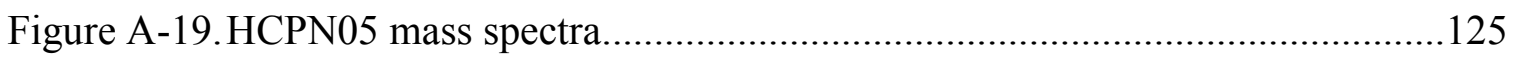

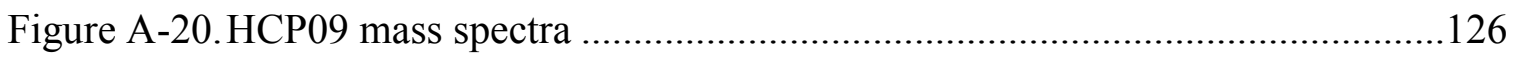

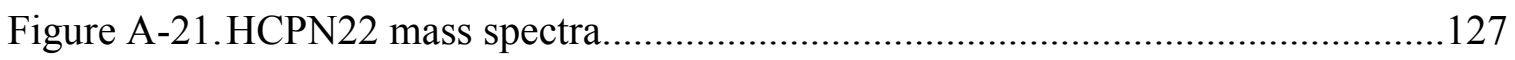

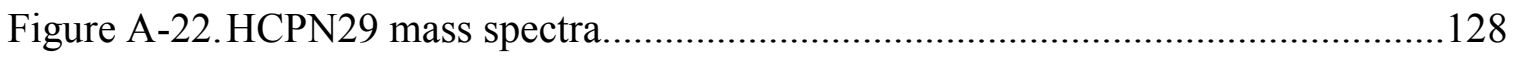

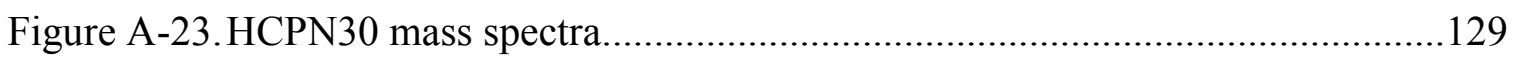

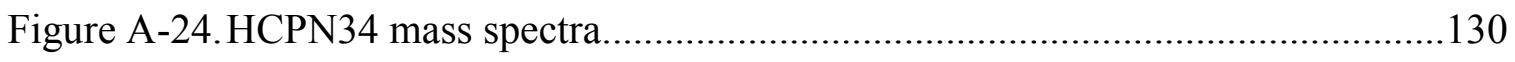

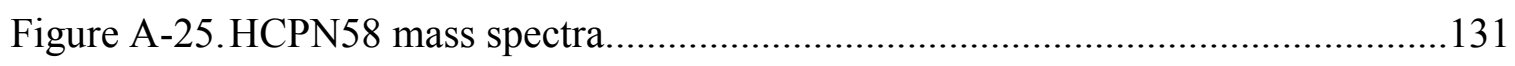

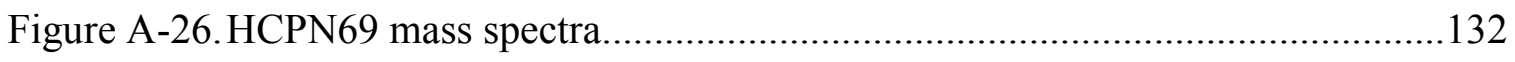

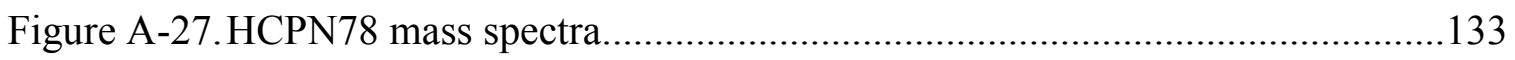

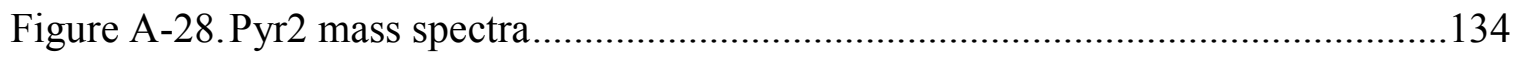

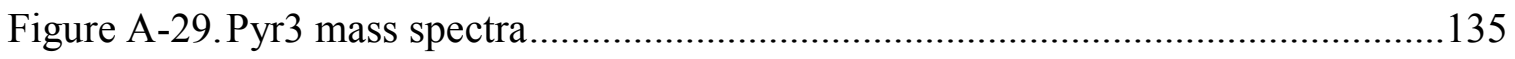

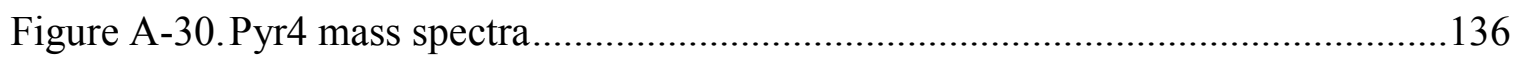

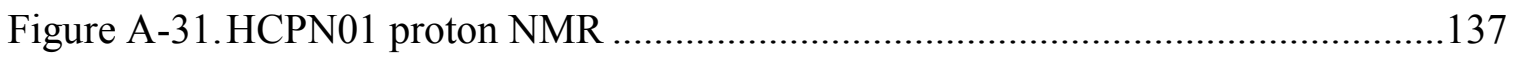

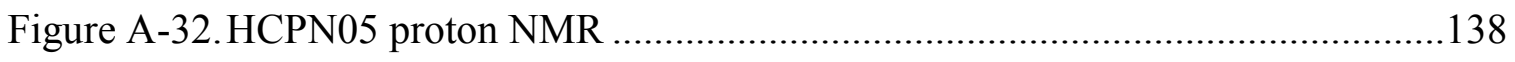

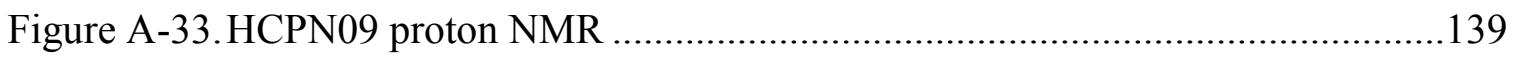

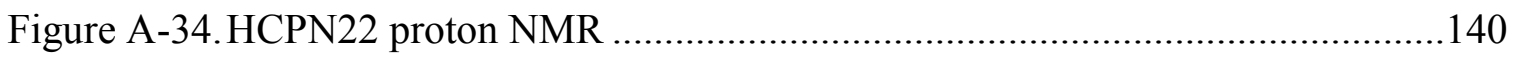

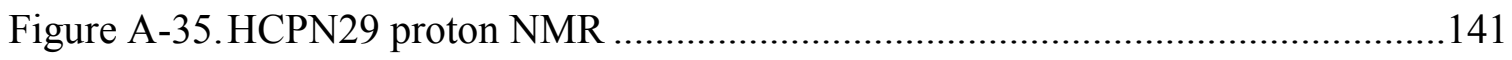

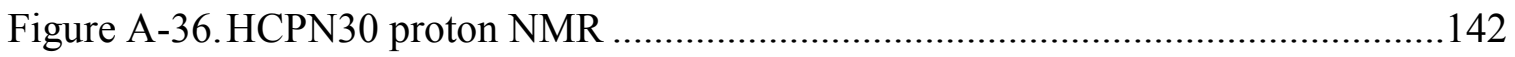

Figure A-37.Expanded upfield section HCPN30 NMR .......................................... 143

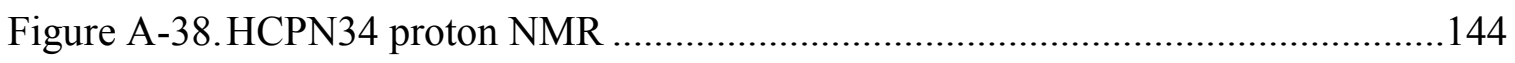

Figure A-39.HCPN58 expanded proton NMR ..................................................... 145

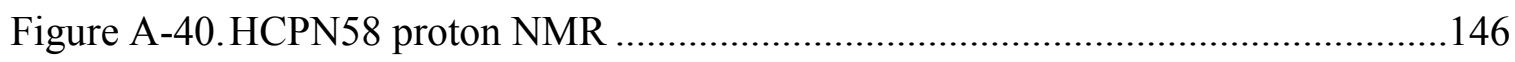

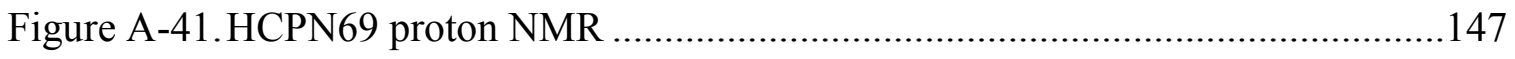

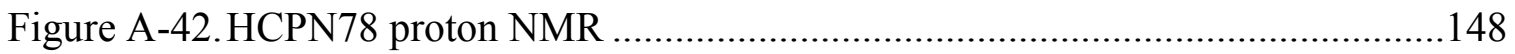


Figure A-43.Expanded HCPN78 proton NMR ....................................................149

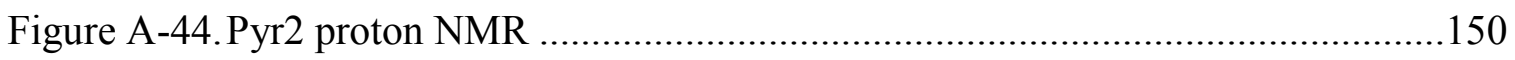

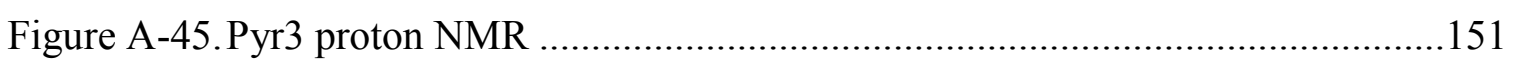

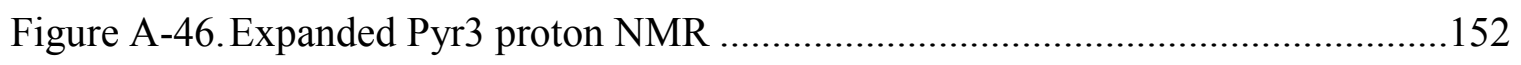

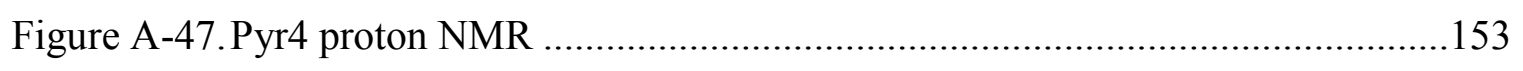

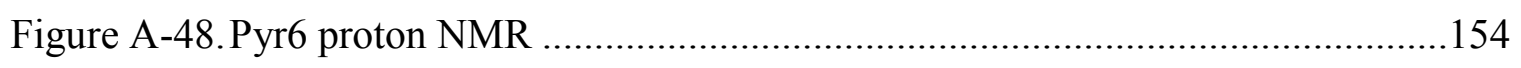




\section{LIST OF ABBREVIATIONS}

$\begin{array}{ll}\text { CNS } & \text { Central nervous system } \\ \text { CNT(s) } & \text { Concentrative nucleoside transporter(s) } \\ \text { DNA } & \text { Deoxyribose nucleic acid } \\ \text { ENT(s) } & \text { Equilibrative nucleoside transporter(s) } \\ \text { F } & \text { F test, statistical measure of significance } \\ \text { LOO } & \text { Leave one out } \\ \text { NBMPR } & \text { S-(4-Nitrobenzyl)-6-thioinosine } \\ \text { NT(s) } & \text { Nucleoside transporter(s) } \\ \text { PMAT } & \text { Poly monoamine transporter } \\ \text { Q }^{2} & \text { Cross-validated (LOO) }{ }^{2} \\ \text { R }^{2} & \text { Correlation coefficient } \\ \text { RNA } & \text { Ribose nucleic acid } \\ \text { S } & \text { Standard deviation of points about the mean of the regression line } \\ \text { SLC28 } & \text { Protein family solute carrier twenty-eight, referring to CNTs } \\ \text { SLC29 } & \text { Protein family solute carrier twenty-nine, referring to ENTs } \\ \text { SLGT1/2 } & \text { Sodium/glucose co-transporter } 1 / 2\end{array}$




\section{CHAPTER 1. INTRODUCTION}

\section{Classification of Nucleoside Transporters}

Nucleosides (Figure 1-1) act as building blocks for DNA, RNA, energetic precursors, regulate energy metabolism, and specifically in the case of adenosine, act as second messengers, regulating a variety of cellular functions. ${ }^{2,3}$ Before the human genome project, our understanding of cells and how they take up nucleosides was based on kinetic experiments in the 1980 's. ${ }^{4}$ The literature still sometimes reflects the nomenclature adopted for the nucleoside transporters at that time. Long before we knew the gene sequences, scientists showed two classes of membrane proteins, one which acted in an equilibrative manner and another which transported purine and pyrimidine nucleosides against the concentration gradient. In addition, further classification divided the equilibrative based on the sensitivity to inhibition by S-(4-nitrobenzyl)-6mercaptopurine riboside (NBMPR), designating the two equilibrative transporters as equilibrative sensitive (es) and equilibrative insensitive (ei). ${ }^{5}$ Similarly, the concentrative transporters were designated as concentrative inhibitor insensitive and utilizing formycin $\mathrm{B}$ as a substrate ( $c i f$ ) or concentrative inhibitor insensitive and utilizing thymidine as a substrate $($ cit $){ }^{5}$ Literature from that era differs in the consensus on the exact number of transporters in each family. Today, thanks to genetic sequencing, seven nucleoside transporters have been identified, four equilibrative and three concentrative, with differing substrate specificities and transport efficiencies. ${ }^{6}$ We now refer to the concentrative family as solute carrier 28 (SLC28) and the equilibrative family as solute carrier 29 (SLC29), with the isoforms designated as ENT1/2/3/4 and CNT1/2/3, respectively. ${ }^{6}$

\section{Nucleoside Transporter Substrate Specificity}

While both families of transporters move nucleosides and synthetic analogues across cellular membranes, they are not evolutionarily related. ${ }^{7}$ Both families evolved early with CNTs being found in eukaryotes and eubacteria. ${ }^{3}$ That evidence, combined with the widespread ENT orthologs in eukaryotes, suggests their vital roles in cellular functions. Numerous studies over the past decades have elucidated their actions in the uptake of endogenous purines, pyrimidines, nucleobases, and chemotherapeutic drugs. ${ }^{2}$

The CNT family operates in a sodium dependent manner against the concentration gradient, with $\mathrm{CNT}^{8}$ and $\mathrm{CNT} 2^{9}$ requiring one to one sodium to transporter stoichiometry for every nucleoside transported, whereas CNT3 uses either two molecules of sodium for every nucleoside or a one each sodium and proton for transfer of one nucleoside molecule across the cell membrane. ${ }^{2}$ The recently published crystal structure of the Vibrio cholerae CNT, which is $36 \%$ and $37 \%$ percent homologous to hCNT1 and hCNT2, respectively and 39\% identical to hCNT3, suggests that the vcCNT functions as a trimer. ${ }^{10}$ It remains to be seen whether that is true and if their hypothesis extends to the human orthologs. hCNT3 is an efficient transporter with broad permeant selectivity. ${ }^{11}$ In contrast hCNT1 transports primarily pyrimidines and their 


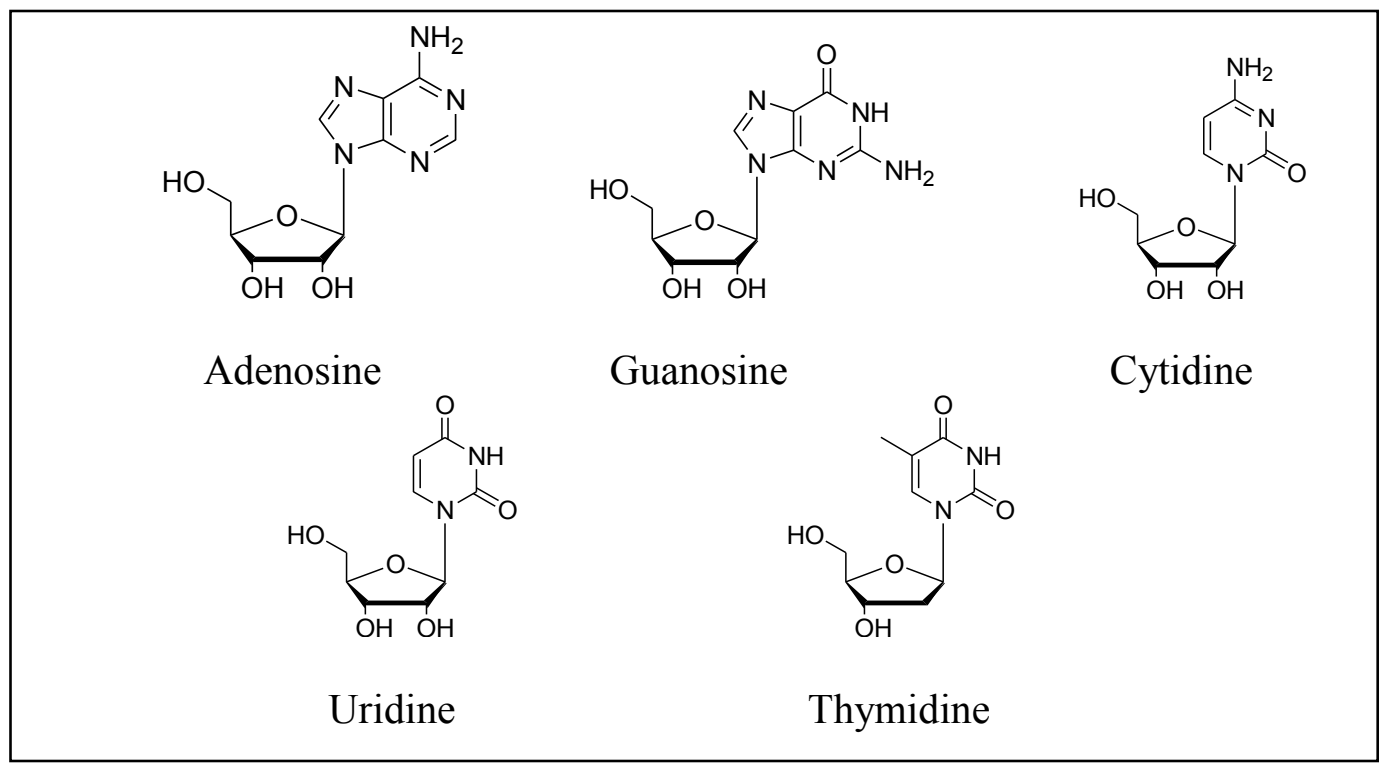

Figure 1-1. Natural nucleosides 
synthetic analogues while hCNT2 transports purines and synthetic purine analogues; however, both transport adenosine (purine) and uridine (pyrimidine), albeit at lower efficiency. ${ }^{9}$ The different concentrative isoforms are primarily located on the plasma membrane, but some cells have been found to have internal hCNT3 and a functional splice variant that is retained within the endoplasmic reticulum has been identified. ${ }^{12}$

In contrast, the equilibrative family moves their substrates across the membrane via facilitated diffusion, either with (ENT3/ENT4) or without (ENT1/ENT2) the aid of a proton. ${ }^{6}$ Historically, hENT2 has proven more resistant to classical inhibitors (Figure 1-2). Both ENT1 and ENT2 have broad permeant selectivity, transporting both purines and pyrimidines, with ENT2 to a lesser extent also transporting nucleobases. ${ }^{6}$ However, recent evidence indicates that hENT1 is also capable of transporting nucleobases, but at a much lower efficiency. ${ }^{13}$ While kinetic experiments in mammalian cells suggest a nucleobase specific sodium-dependent mechanism for cellular uptake, to date no human nucleobase transporter proteins have been isolated. ${ }^{2}$ As such, this highlights the importance of the nucleoside transporters for both nucleoside and nucleobase salvage and in the uptake of drugs targeting biological pathways utilizing them.

Both hENT1 and hENT2 are primarily found on the plasma membrane, but are also found on the nuclear membrane. In addition, ENT1 has been found on the mitochondria. ${ }^{6}$ The presence in mitochondrial membranes does not appear to be general across mammalian species. For example, rats do not express ENT1 on their mitochondrial membrane. ${ }^{6}$ ENT3, which is found primarily on the lysosome, endosome, and mitochondria, has not to date been reported as expressed on the plasma membrane and appears to function primarily as an intracellular transporter. ${ }^{6}$ It transports purines, pyrimidines, and nucleobases, but not hypoxanthine, which ENT2 transports. ${ }^{6}$ In addition, the reported $K_{m}$ s for endogenous substrates are higher in comparison to ENT1 and ENT2. ${ }^{6}$ This difference in $K m$ could potentially exist as a regulation mechanism so that as ENT1 and ENT2 take up nucleosides, they are not disproportionately taken up by the lysosomes before they can be utilized.

Lastly, differing significantly in sequence similarity and substrates, ENT4 is primarily located on the plasma membrane and transports monoamines at physiological $\mathrm{pH}$. Wang et $_{\mathrm{al}} \mathrm{l}^{14}$ postulates that ENT4 is more closely related in function under normal physiological conditions to the PMATs (poly monoamine transporters), transporting serotonin and dopamine in the brain, with adenosine and other nucleoside transport being incidental. However, sufficient data exists that under ischemic acidic conditions, the local $\mathrm{pH}$ can drop into a range in which hENT4's transport of adenosine and other nucleosides would be significant. ${ }^{15,16}$

\section{Transporter Structure and Biological Distribution}

Both transporter families are transmembrane proteins, but they differ in length. While we do not yet have crystal structures to definitively elucidate their biological 


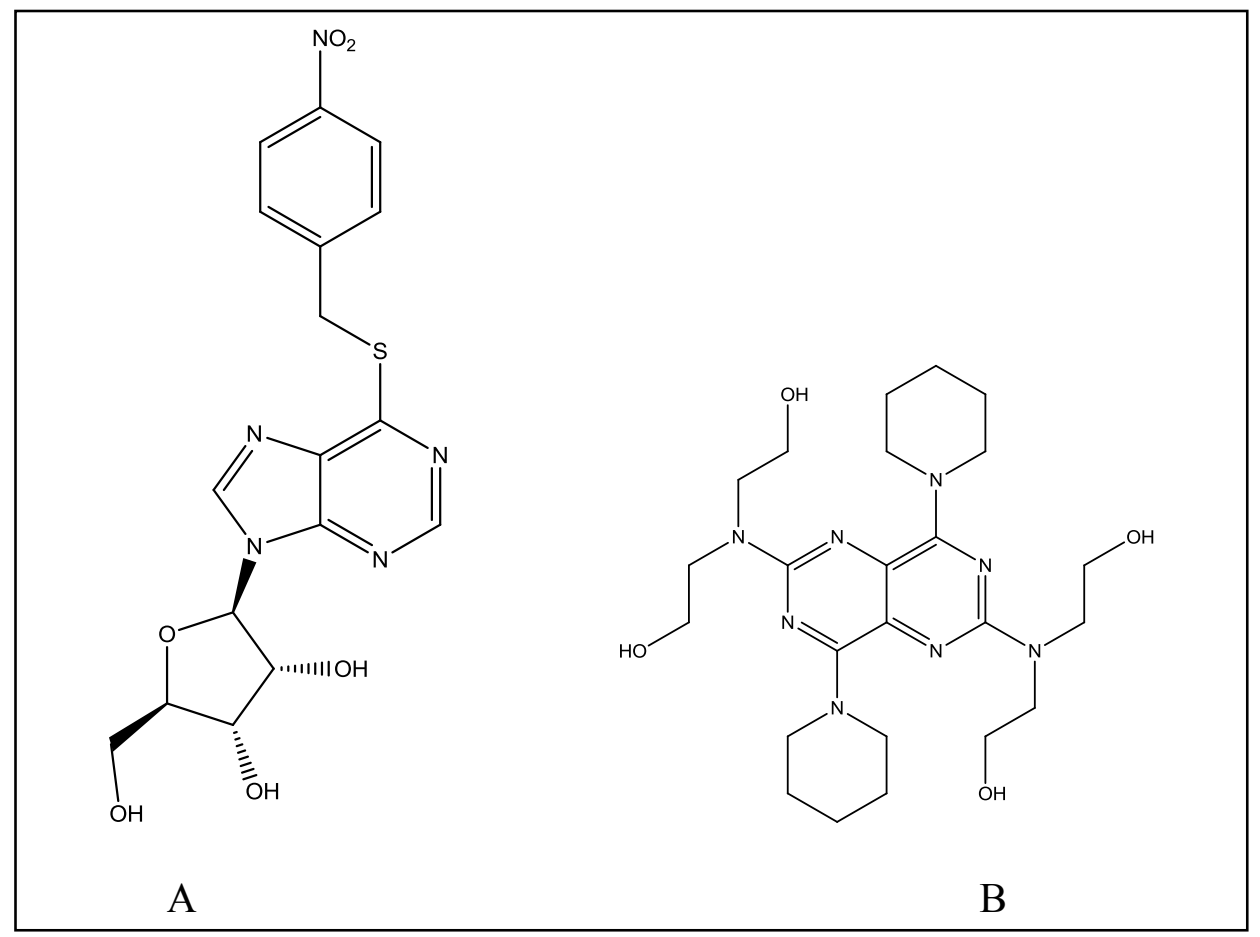

Figure 1-2. Classic nucleoside inhibitors

Note: (A) S-(4-Nitrobenzyl)-6-thioinosine (NBMPR), (B) dipyridamole 
conformations, similarity to other protein families and a number of molecular biological experiments have outlined their theoretical structures.

In 1997, Young et al. cloned the human gene for CNT1 $1{ }^{17}$ Located on chromosome 15 q25-26, it is $83 \%$ homologous to its rat ortholog, and codes for a 690 amino acid protein. ${ }^{17}$ Originally, scientists proposed a fourteen transmembrane model, ${ }^{18}$ but later revised the model to thirteen transmembrane regions. ${ }^{19}$ However, in 2009 , another group put forth a fifteen transmembrane model based on a newer molecular modeling method..$^{20}$ In short, the exact topologies of the CNT transporters remain unverified.

Not long after, the 659 amino acid protein for hCNT2 was cloned and localized to chromosome 15q15, followed the 691 amino acid protein for hCNT3, on chromosome $9 \mathrm{q} 22.2^{3}$ The human isoforms share less similarity with each other than they do with other species orthologs. For example, hCNT1 is $71 \%$ homologous to hCNT2, but as mentioned, $83 \%$ rCNT1. hCNT3 shares even less homology at $48 \%$ and $47 \%$ sequence similarity to hCNT1 and hCNT2, respectively. ${ }^{3}$ This, in addition to their presence in bacteria, suggests highly conserved function, vital to cellular processes.

The sequences of hENT1 and hENT2 were determined in 1996, but hENT3 and hENT4 were not sequenced for close to a decade after that. ${ }^{7}$ Both hENT1 and hENT2 are 456-amino acid proteins sharing 46\% homology. ${ }^{7}$ The intracellular hENT3 is coded by a 475 amino acid sequence and shares only $29 \%$ homology with hENT1. ${ }^{7}$ The last protein in the ENT family, and arguably the most evolutionarily distant, is coded by 530 amino acid protein and is only $18 \%$ homologous to hENT1. Experiments have confirmed a proposed 11 transmembrane topology, ${ }^{7}$ highlighting another difference between the CNT and ENT families.

Together these two families function to maintain homeostasis. As such, they have different biological distributions. Table 1-1 outlines the predominant tissues in which each of the transporters is found.

It is not uncommon to find ENTs on one side of a cell and CNTs on another side, especially in organs such as the intestines, liver, kidneys, and brain. Figure 1-3, which was published in a recent review illustrates some of the arrangements of nucleoside transporters found to date. ${ }^{2}$

While altered expression of nucleoside levels have been shown in a number of disease states, with only one exception, no specific pathology is directly linked to nucleoside transporter mutations. Hystiocytosis syndrome and Rosai-Dorfman disease are two lysosomal storage diseases with overlapping phenotypes which are directly linked to germ line mutations of hENT3. ${ }^{21}$ The mutations alter the function of hENT3 or result in a nonfunctional protein. In vitro studies suggest hENT3 plays a role in regulating cell growth and proliferation and that in its absence cells experience aberrant growth and proliferation. ${ }^{21}$ Still, the exact method of pathogenesis requires further elucidation.

Table 1-2 lists known mutations, many of which alter permeant kinetics. Their effects on disease and drug metabolism are still being explored, but preliminary studies indicate that 
Table 1-1. Nucleoside transporter tissue distribution in humans

\begin{tabular}{ll}
\hline Transporter & \multicolumn{1}{c}{ Tissue } \\
\hline ENT1 & $\begin{array}{l}\text { Widely expressed. Found on the plasma membrane } \\
\text { of most tissues, also on nuclear membranes and } \\
\text { mitochondrial membranes. } \\
\text { WNT2 } \\
\text { Widely expressed, with more abundance in skeletal } \\
\text { tissues. Found primarily on plasma membranes, but } \\
\text { also detected on some nuclear membranes. }\end{array}$ \\
ENT3 & $\begin{array}{l}\text { Widely expressed, intracellular. } \\
\text { ENT4 }\end{array}$ \\
CNT1 & $\begin{array}{l}\text { Specialized epithelial cells: kidney, liver, intestines, } \\
\text { plasma membrane. }\end{array}$ \\
CNT2 & $\begin{array}{l}\text { Plasma membrane, heart, skeletal muscle, liver, } \\
\text { kidney, intestine, pancreas, placenta, and brain. }\end{array}$ \\
CNT3 & $\begin{array}{l}\text { Mostly plasma membrane, but intracellular in some } \\
\text { cells. Located in most abundance in mammary } \\
\text { glands, pancreas, bone marrow, trachea, and } \\
\text { intestines, but found throughout body. }\end{array}$ \\
\hline
\end{tabular}

Reprinted with permission from Elsevier. Young, J. D.; Yao, S. Y. M.; Baldwin, J. M.; Cass, C. E.; Baldwin, S. A. Molecular Aspects of Medicine 34 (2013) 529-547. 
(A) Intestine

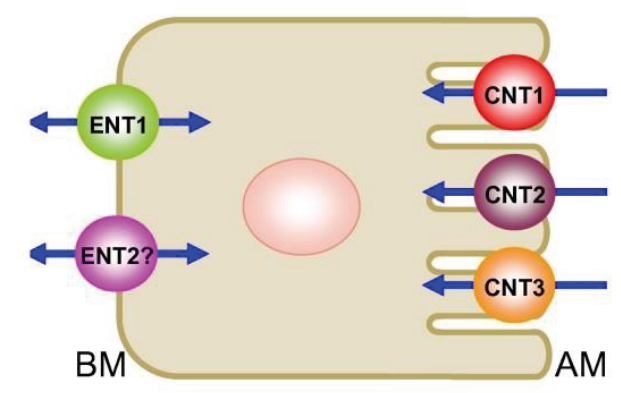

(B) Kidney

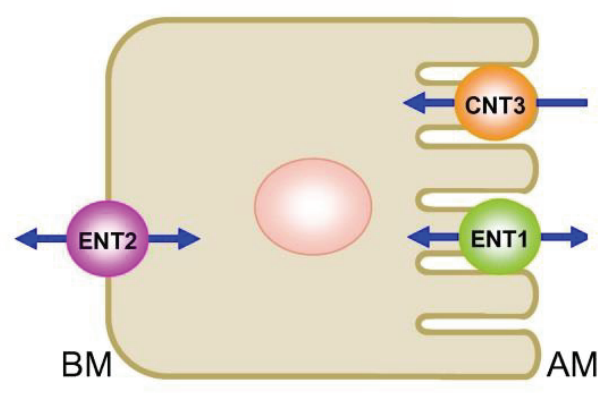

(C) Liver

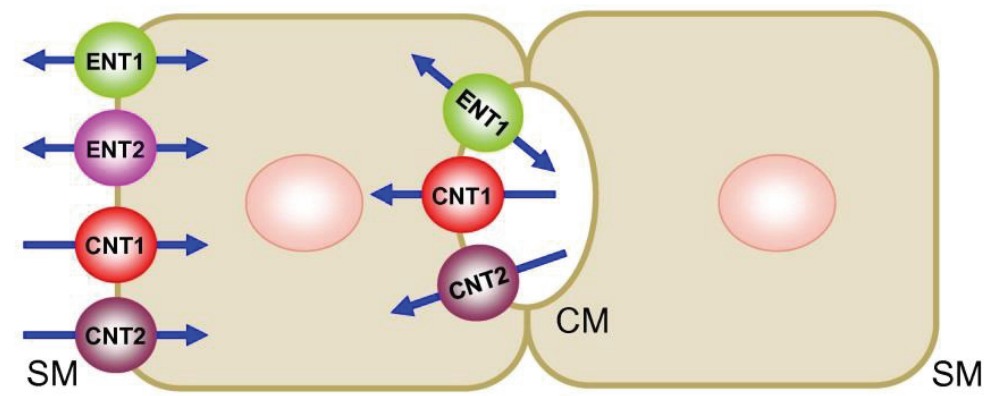

(D) Brain
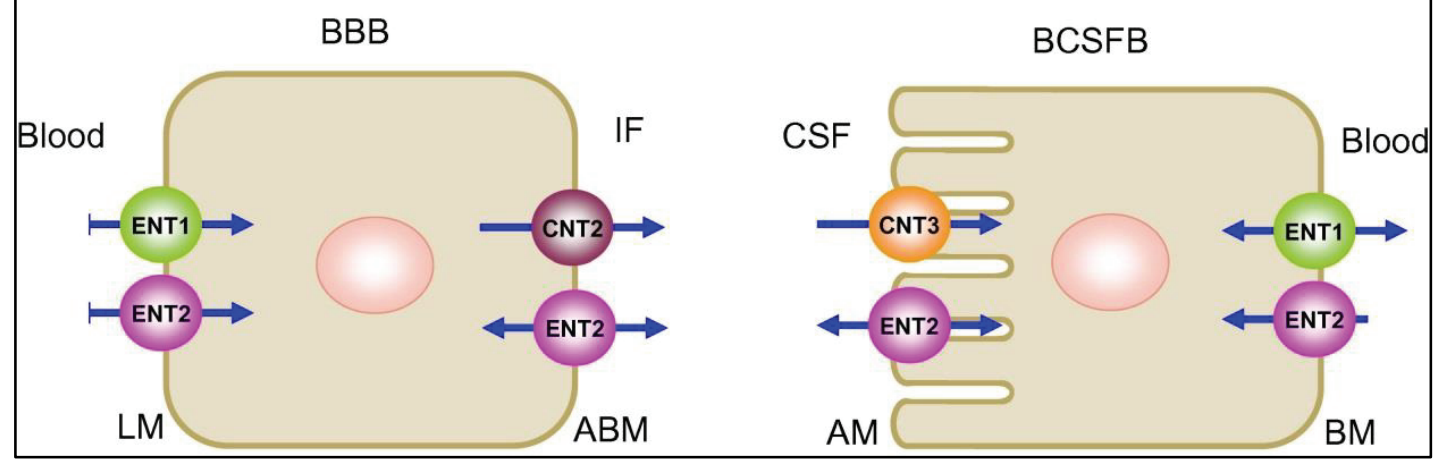

Figure 1-3. Graphical depiction of human nucleoside transporter distributions

Note: Abbreviations used in this Figure are: (A, B) apical membrane (AM), basolateral membrane (BM); (C) canalicular membrane (CM), sinusoidal membrane (SM); (D) blood-brain barrier endothelial cells (BBB), blood-cerebrospinal fluid barrier epithelial cells of the choroid plexus (BCSFB), interstitial fluid (IF), cerebrospinal fluid (CSF), abluminal (IF-facing) membrane of the BBB (ABM), luminal (blood-facing) membrane of the BBB (LM), apical (CSF-facing) membrane of the BCSFB (AM), basolateral (blood-facing) membrane of the BCSFB (BM).

Reprinted with permission from Elsevier. Young, J. D.; Yao, S. Y. M.; Baldwin, J. M.; Cass, C. E.; Baldwin, S. A. Molecular Aspects of Medicine 34 (2013) 529-547. 
Table 1-2. Current known nucleoside transporter mutations

\begin{tabular}{|c|c|}
\hline Transporter & Mutations \\
\hline ENT1 & $\begin{array}{l}\text { - Single missense point mutation at glycine } 24 \text { (cancer cell } \\
\text { line })^{37}\end{array}$ \\
\hline ENT2 & $\begin{array}{l}\text { - Exon } 4 \text { splice variant leading to N-terminally truncated, } \\
\text { inactive protein }(\mathrm{HNP} 36)^{2} \\
\text { - Exon } 9 \text { splice variant leading to C-terminally truncated, } \\
\text { inactive protein }(\mathrm{ENT} 2 \mathrm{~A})^{2}\end{array}$ \\
\hline ENT3 & $\begin{array}{l}\text { - } \mathrm{M} 116 \mathrm{R} \\
\text { - } \mathrm{G} 427 \mathrm{~S} \\
\text { - } \mathrm{G} 437 \mathrm{R} \\
\text { - } \mathrm{T} 449 \mathrm{R} \\
\text { - } \text { various frameshift mutations } \\
\text { All abolish or reduce transport activity, cause mistrafficking, } \\
\text { or protein instability } \\
\text { Associated with H, FHC and PHID syndromes and Rosai- } \\
\text { Dorfman disease }{ }^{2,21}\end{array}$ \\
\hline ENT4 & - None reported to date \\
\hline CNT1 & $\begin{array}{ll}- & \mathrm{S} 546 \mathrm{P} \\
\text { - } & \mathrm{V} 189 \mathrm{I} \\
\text { - } & 1153 \mathrm{del}^{38}\end{array}$ \\
\hline CNT2 & $\begin{array}{ll}\text { - } & \mathrm{F} 355 \mathrm{~S} \\
\text { - } & \mathrm{E} 385 \mathrm{~K}^{39}\end{array}$ \\
\hline CNT3 & $\begin{array}{l}\text { - Splice variant CNT3ins lacks the N-terminal } 69 \text { residues and } \\
\text { is retained in the endoplasmic reticulum (still functional) } \\
\text { - Gly367Arg }\end{array}$ \\
\hline
\end{tabular}


variants can contribute to altered uptake of chemotherapeutics as well as endogenous substrates. Likewise, it is not fully understood why so many cells express various transporters, resulting in overlapping permeant selectivity. Perhaps, as they tie in directly to the scavenging of molecules required for DNA replication, evolution resulted in redundancies, which aid in cellular survival, but a great deal of further research is needed to confirm or refute that speculation.

\section{Nucleoside Transporters and Species Variations}

Animal models have elucidated a number of potential roles that nucleoside transporters play. In Drosophila fruit flies, DeENT2 was shown to regulate cognitive learning and that the null mutant was lethal. ${ }^{22}$ In contrast a null ENT1 mouse model demonstrated a link between alcohol sensitivity and ENT1, suggesting that it played a role in intoxication. ${ }^{23}$ Otherwise, ENT1 null mice appeared healthy. While these and other findings might hold true in humans, one cannot assume that every animal model directly correlates to human biology.

Despite the high degree of similarity and function of the nucleoside transporters, species differences do exist. For example, rat ENT1 and ENT2 are more resistant to inhibition by the same molecules that inhibit the human orthologs at nanomolar concentrations. ${ }^{7}$ When utilizing cells for assays, rat or mouse results may not translate to human activity and vice versa. Even tissue distributions vary from species to species. For example, mice and rats, both rodents, express different amounts of CNT2 in their livers, implying potentially vastly different pharmacokinetics of nucleoside chemotherapeutic drugs. ${ }^{24}$ Rats expressed high amounts of hepatic rCNT2, but mice had no measurable hepatic mCNT2. ${ }^{24}$ When designing animal in vivo studies of nucleoside transporters, one must keep species variance in mind as results may not always translate to human systems.

\section{Known Inhibitors of Nucleoside Transporters}

Broadly speaking, known nucleoside transporter inhibitors can be divided into two categories: nucleoside analogue inhibitors and non-nucleoside analogues. Of all the transporters, hENT1 appears to be most easily inhibited by a variety of molecules. It should also be noted that the literature identification of inhibitors and reported potencies can vary depending on the species of cell used and even on cell type.

\section{hENT Inhibitors}

Belonging to the 9-ß-D-ribofuranosyl purine class (Figure 1-2) NBMPR was identified in 1974 as a potent inhibitor of hENT1, inhibiting at nanomolar concentrations and is often used as the reference standard today. ${ }^{25}$ Due to mutagenicity, immunosuppressive properties, ${ }^{26}$ and poor pharmacokinetics it has limited potential as a clinical agent. ${ }^{27}$ In the nearly four decades since, a wide variety of nucleoside analogues and small molecules have been found to inhibit hENT1, although potency varies. 
NBMPR belongs within the category of nucleoside analogues. Within that category, one also finds cyclic adenosine mono-phosphate (cAMP) derivatives. ${ }^{28}$

A number of the small molecule inhibitors fit into either purine or pyrimidine analogues, which is logical as hENT1 transports these and the functionality would be the source of affinity for the molecules. Table 1-3 lists some examples of the variety of molecules that have been found to inhibit hENT1.

Table 1-3 is by no means an exhaustive list, but rather represents examples of the most potent chemical classes identified to date. Some of the compounds listed, such as dipyridamole and dilazep also inhibit hENT2, albeit with Kis several fold higher. ${ }^{29}$ Hammond et al. identified draflazine analogues (Figure 1-4) which showed hENT2 selectivity. ${ }^{30}$ In addition, a number of dipyridamole analogues were synthesized which demonstrated hENT2 selectivity. ${ }^{31}$ While these compounds are promising, the identification of hENT2 potent and selective inhibitors remains a challenging obstacle to fully exploring the cell signaling role of this transporter.

Far fewer studies have been conducted on hENT3 due to its intracellular location and the difficulty of isolating functional proteins. The studies that have been conducted demonstrate that it is resistant to traditional hENT inhibitors and its functionality is $\mathrm{pH}$ dependent. $^{32}$

Like hENT3, hENT4 nucleoside transport is $\mathrm{pH}$ dependent and weakly inhibited by traditional inhibitors like NBMPR and dipyridamole. ${ }^{16}$ However, recently published work showed a series of dipyridamole analogues which demonstrated hENT4 selectivity and potency. ${ }^{1}$ Figure 1-5 illustrates the structure of the most potent analogue WL30. ${ }^{1}$

\section{hCNT Inhibitors}

In contrast to the equilibrative transporters, there have been far fewer inhibitors identified for the concentrative transporters. One group identified a class of purine nucleoside derivatives with 8-position modifications as selective hCNT2 inhibitors (Figure 1-6). ${ }^{33}$

In 2014 another group reported that several chemotherapeutic agents inhibit nucleoside transporters, including concentrative transporters, at varying micromolar concentrations (Table 1-4).

As Figure 1-7 shows, these are non-nucleoside structures, but their toxicity and non-specific action make them poor tools for studying nucleoside transporters. Another compound, traditionally used as a pan-inhibitor of concentrative nucleoside transporters is phlordizin (Figure 1-8). ${ }^{34}$ A natural product isolated from apple wood and other plants, it also inhibits sodium/glucose co-transporters one and two (SGLT1 and SGLT2). ${ }^{35}$ Its inhibitory Kis of the CNTs measures at or greater than $17 \mu \mathrm{M}$ for all of the isoforms, highlighting its lack of potency and selectivity. ${ }^{34,36}$ Of the three CNT transporters, it demonstrates the highest potency against hCNT3. With that in mind, Gupte et al. 
Table 1-3. Survey of non-nucleoside inhibitors of ENT1

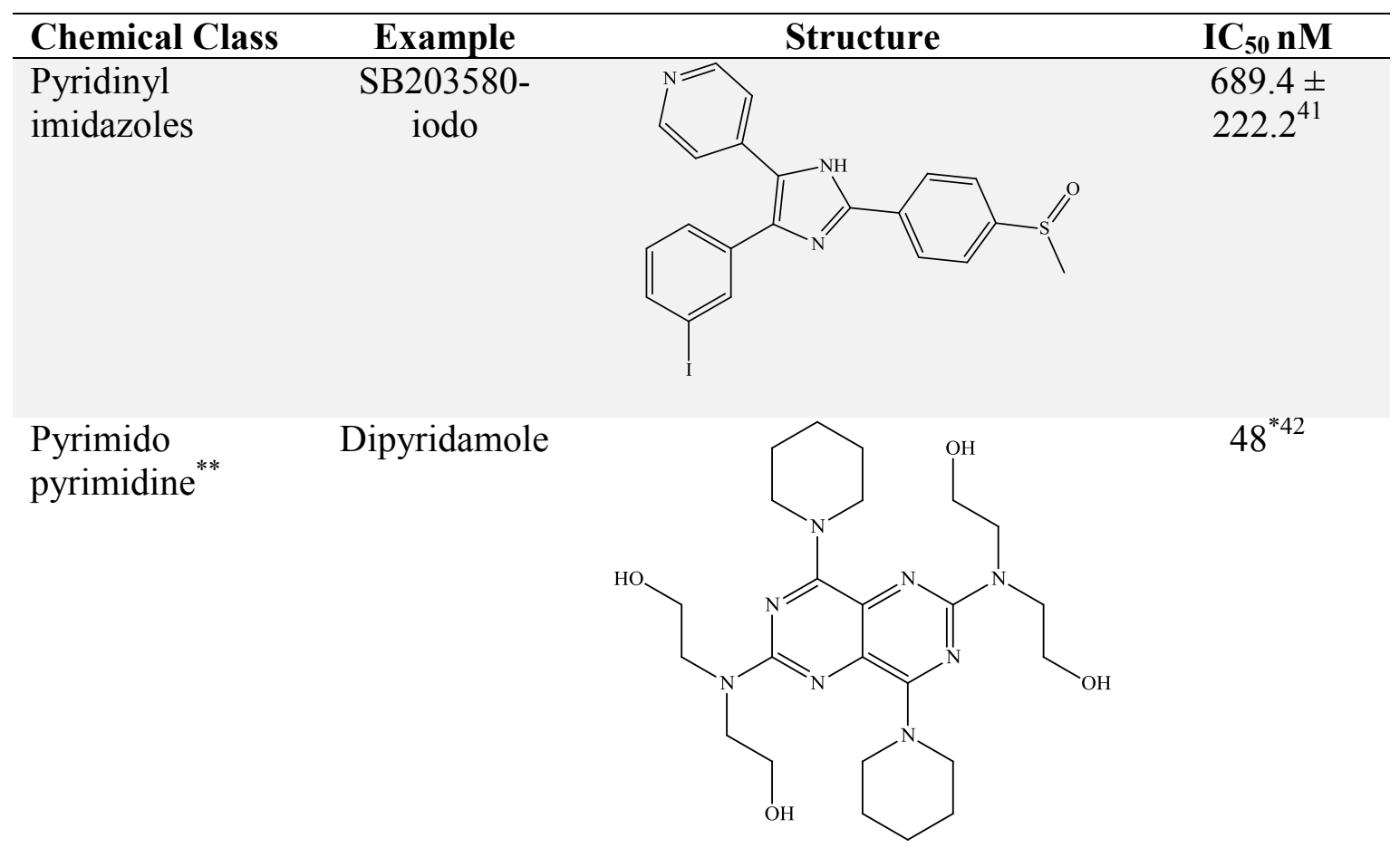

Thiazolindedion Troglitazone es

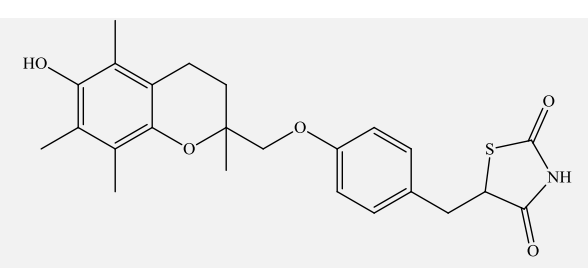

$2350 \pm 350^{43}$

Xanthine

Febuxostat

Oxidase<smiles>Cc1nc(-c2ccc(OCC(C)C)c(C#N)c2)sc1C(=O)O</smiles>

$4100^{* 44}$

Inhibitors

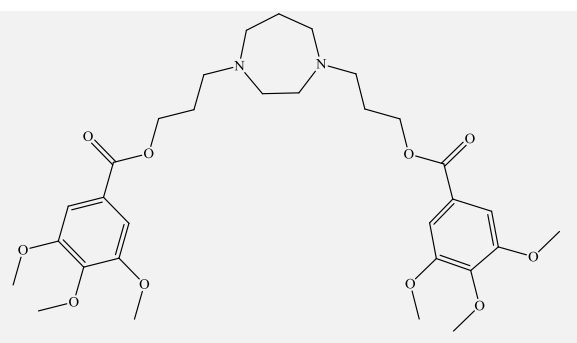

$18.7 \pm 2^{42}$

Diazepane
Piperazines

Dilazep 
Table 1-3. Continued

\begin{tabular}{llll}
\hline Chemical Class & Example & Structure & $\mathbf{I C}_{\mathbf{5 0}} \mathbf{n M}$ \\
\hline Diazepane & Dilazep & $18.7 \pm 2^{42}$
\end{tabular}

Dihydropyridines Nimodipine

(Calcium channel

blockers)

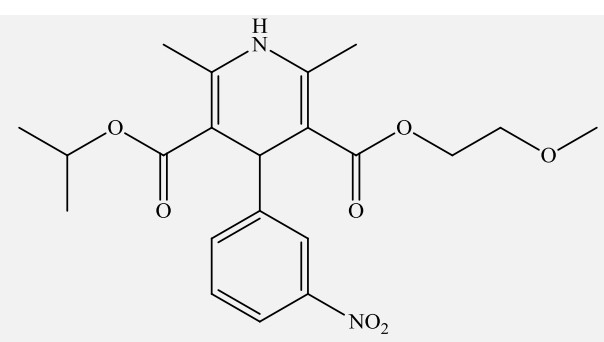

$200^{45}$

Note: *reported $K i$ value. $* *$ Includes pteridine derivatives ${ }^{46}$ 


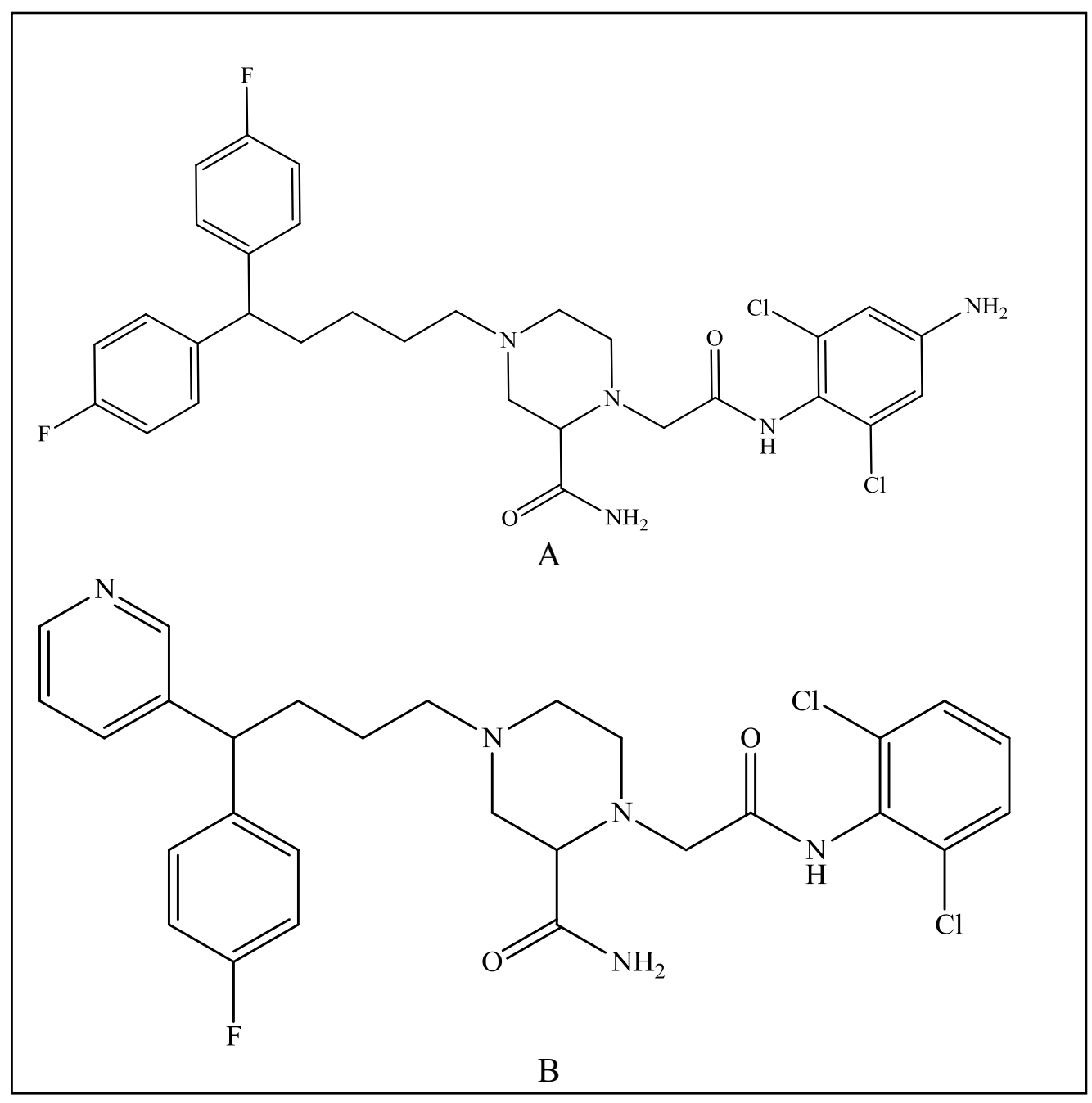

Figure 1-4. (A) Draflazine and (B) soluflazine

Note: Draflazine is hENT1 selective while soluflazine shows greater hENT2 affinity. ${ }^{30}$ 


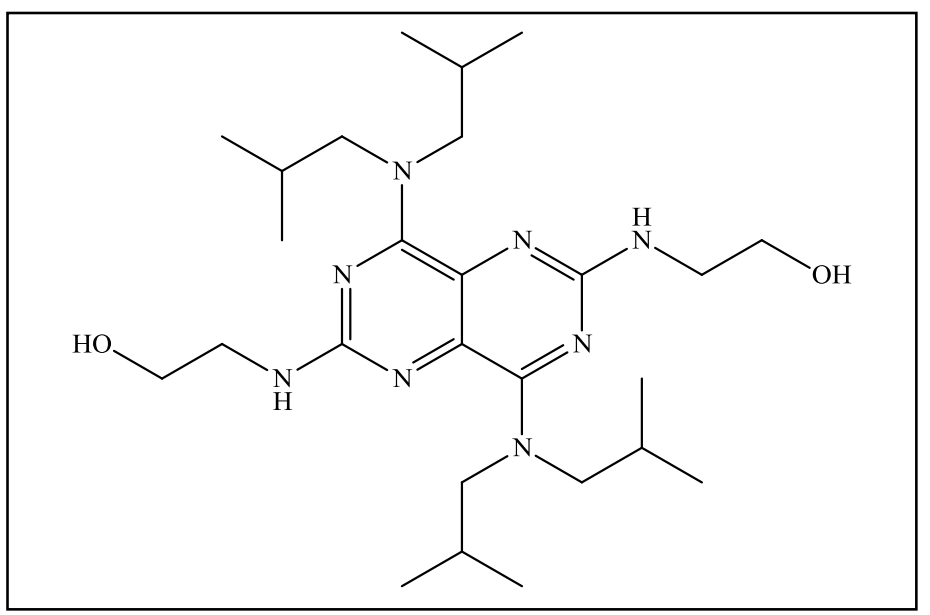

Figure 1-5. WL30 a potent and selective hENT4 inhibitor

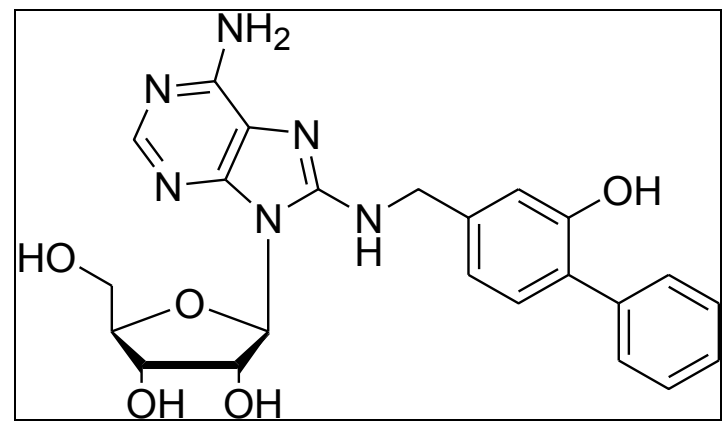

Figure 1-6. 8-position modified purine analogue

Table 1-4. Micomolar/ $\mathrm{L} \mathrm{IC}_{50}$ of uridine transport in yeast

\begin{tabular}{lccc}
\hline Transporter & Erlotinib & Gefitinib & Vandetanib \\
\hline hENT1 & $34 \pm 6$ & $14 \pm 2$ & $11 \pm 1$ \\
hENT2 & $>300$ & $>300$ & $89 \pm 17$ \\
hCNT1 & $160 \pm 20$ & $37 \pm 11$ & $64 \pm 17$ \\
hCNT2 & $>300$ & $>300$ & $82 \pm 4$ \\
hCNT3 & $11 \pm 1$ & $>300$ & $28 \pm 9$ \\
\hline
\end{tabular}

Reprinted with permission from Elsevier. Damaraju, V. L.; Scriver, T.; Mowles, D.;

Kuzma, M.; Ryan, A. J.; Cass, C. E.; Sawyer, M. B. Clinical Cancer Research 2014, 20, 176. 


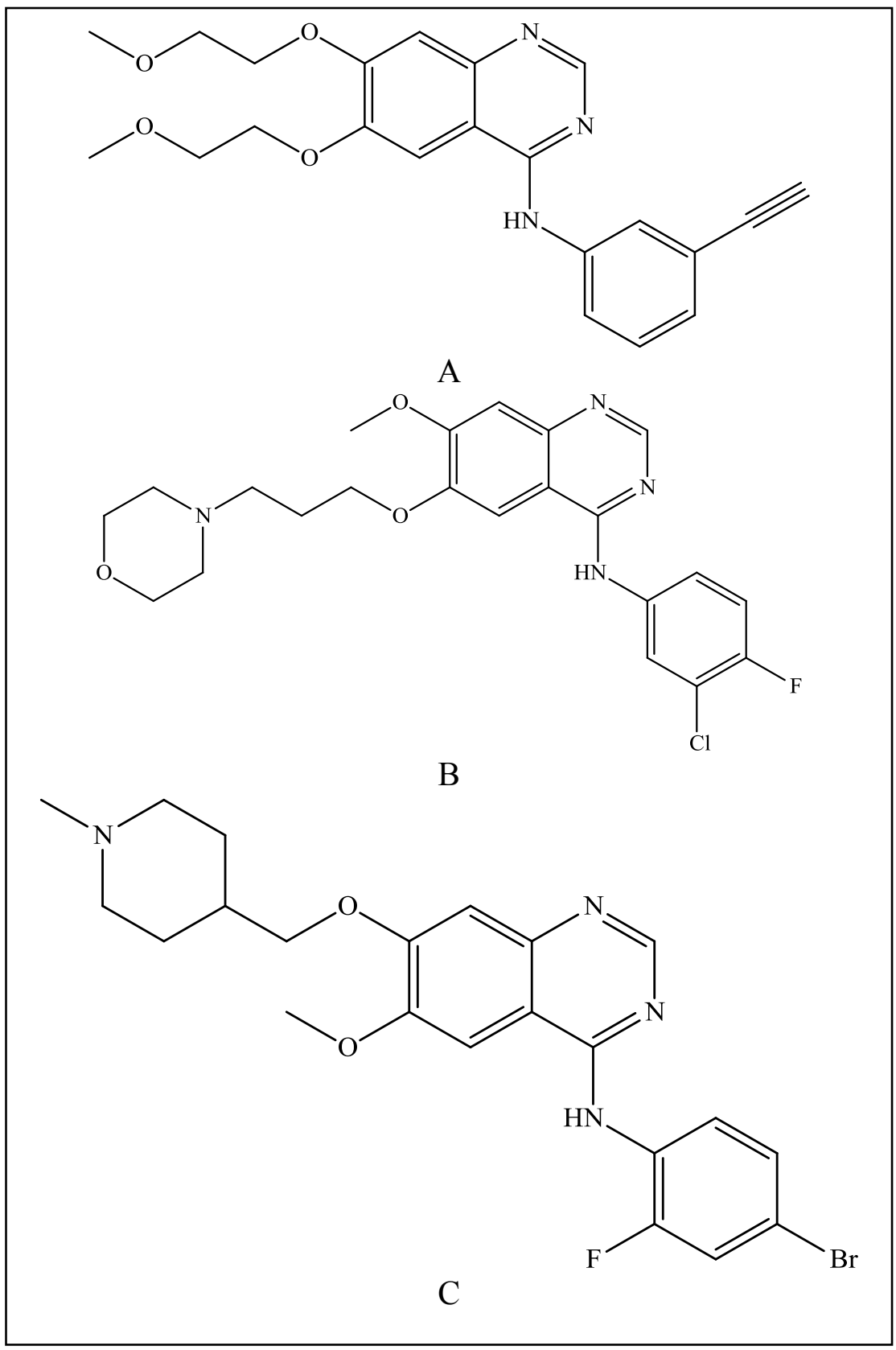

Figure 1-7. Chemotherapy agents with nucleoside transporter inhibitory activity Note: (A) Erlotinib (B) Gefitinib (C) Vandetanib 


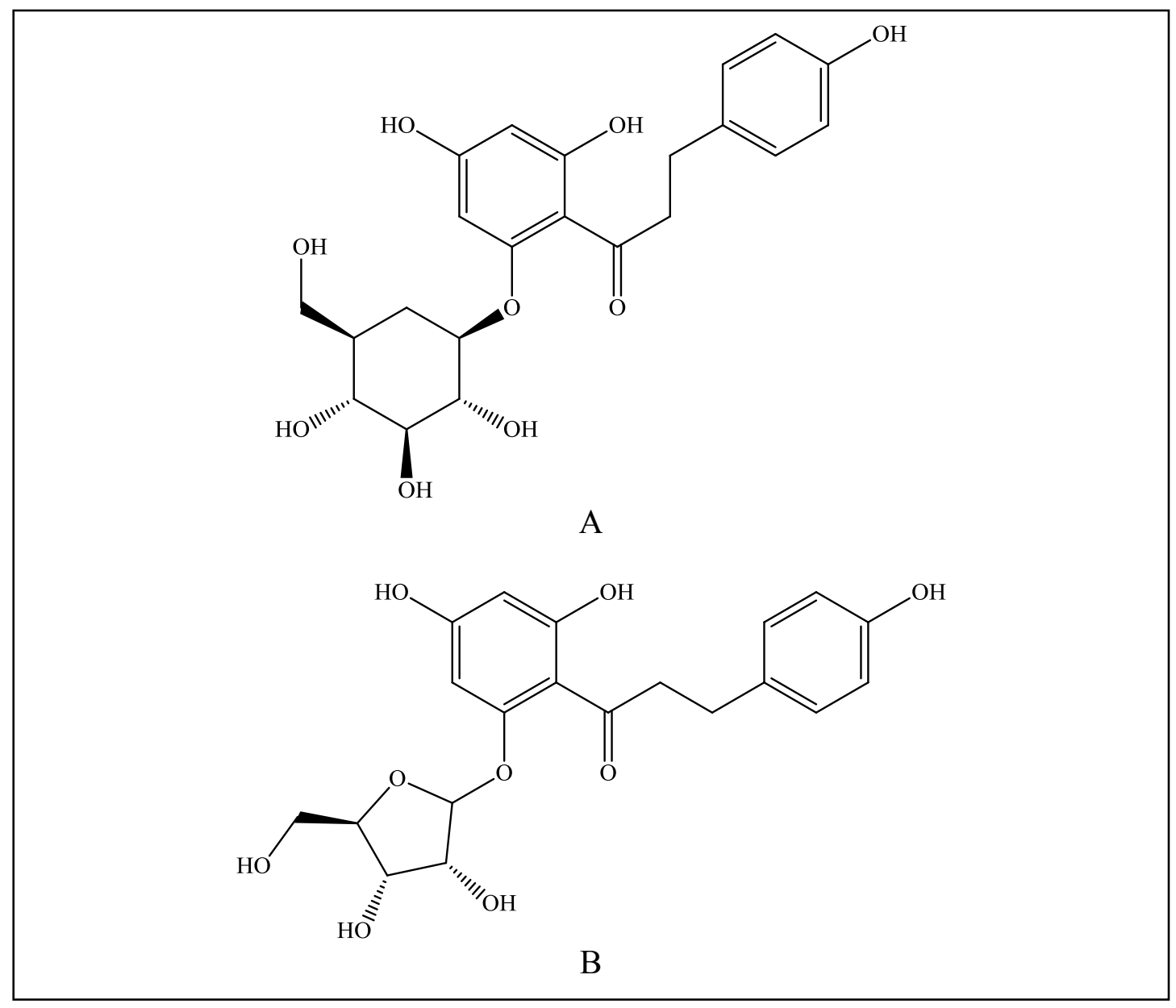

Figure 1-8. (A) Phlorizin (B) phlorizin analogue 
synthesized a series of analogues, successfully making a derivative with higher potency. ${ }^{34}$ With a $K i$ of $2.88 \mu \mathrm{M},{ }^{34}$ it shows a marked increase in potency, but the furanose ring is less than optimal for drug design.

\section{Nucleoside Transporter Inhibitor Applications and Purpose of Study}

Small molecule, selective inhibitors can assist in our examinations of cell signaling and the roles nucleoside transporters play on homeostasis as well as pathogenic states. Many of the drugs currently used as cancer chemotherapy agents, ${ }^{48}$ in addition antiretrovirals ${ }^{49}$ are transported by nucleoside transporters. In addition, it has been well established that nucleoside transporters modulate extracellular adenosine and during ischemic events extracellular adenosine can effect cell survival during reperfusion. ${ }^{50,51}$ While the exact molecular mechanisms of this are still being elucidated, it suggests that nucleoside transporters can be utilized for uses such as cardio-, neuro-, or renal protection during ischemic events.

Cancer chemotherapy, in which nucleoside transporters play a role in both uptake and efflux of drugs and endogenous nucleosides, is hampered by changes in cellular expression and toxicity to healthy cells. The use of viral vectors, while effective to selectively target cancer cells, has limited in vivo efficacy due to poor spread and replication, however a recent study showed that use of nucleoside transporter inhibitors could increase their potency. ${ }^{52}$ The oHSV1 vector is transfected to express an oncolytic gene. The nucleoside inhibitors likely increase their efficacy by preventing the salvage of nucleosides and nucleobases. The viral vector uses up the available nucleosides within the cell for replication, leaving the cancer cell short on the critical components necessary for DNA replication.

Furthermore, adenosine is implicated in modulating immune function and inflammation. ${ }^{53,54}$ As such, further exploration of the role of nucleoside transporters in modulating inflammation could bring new understanding to the field.

Humans and other mammals can synthesize nucleosides in most of our tissues, whereas many bacteria rely on salvage. Species differences also allow for selective targeting of non-mammalian transporters. Nucleoside transporters are a novel target for those focusing on pathogenic agents, such as malaria or bacteria. ${ }^{55,56}$

Without selective, potent small molecule inhibitors, we cannot further our knowledge of vital cell signaling pathways. Likewise, the potential applications cannot be explored. While there are a number of small molecule hENT1 inhibitors, ones that enter the CNS are still needed, and the other transporters lack diverse, potent, and selective molecular inhibitors.

It was the goal of this study to utilize computer-aided drug design, synthesis, and cell-based screening to identify novel scaffolds for each of the nucleoside transporters, with the exception of hENT3, for further study. 


\section{CHAPTER 2. COMPUTER MODELING STUDIES}

\section{Introduction}

There are two primary approaches to computer-aided drug design and discovery: structure based and ligand based. Structure based methods require a crystal structure or a homology model based on a similar protein, or a structure determined by other methods such as NMR. In ligand based methods, the researcher aims to correlate structural features of active and inactive molecules with biological activity. While there are a number of methods, this study focused on three quantitative structure-activity relationship (QSAR) methods: comparative field analysis (CoMFA), ${ }^{57}$ comparative similarity indices analysis (CoMSIA), ${ }^{58}$ and pharmacophore (3D QSAR) ${ }^{59}$ The concept of a pharmacophore, or structure features of a molecule required for bioactivity, was developed by Lemont Kier, and has greatly advanced in the four decades since he first described it. Pharmacophore 3D QSAR can be used alone to digitally screen databases for potential bioactive molecules or in concert with CoMSIA and CoMFA as an alignment method. ${ }^{60}$ While, these methods were primarily used for the selection of compounds for screening against the concentrative transporter, in 2012 a crystal structure of a Vibrio cholera transporter was published. ${ }^{10}$ The $V$. cholera and hCNT1 shared enough similarity that Dr. Hemantkumar Deokar of our laboratory created a hCNT1 homology model (unpublished). Both the V. cholera crystal structure and homology model were used by him to select two sets of the compounds presented in this study.

A number of key assumptions underlie 3D QSAR methods. ${ }^{61}$

1. The ligands all bind at the same location and in the same mode.

2. The conformation(s) of the molecules in the database are the bioactive conformations.

3. There is negligible difference in entropic factors.

4. Biological activity results primarily from enthalpic contributions.

5. That the biological measurements are made without error.

6. The system is at equilibrium.

7. Solvent effects, transport and diffusion are not important to biological activity.

Understanding the underlying assumptions can help to interpret why a model performs poorly and perhaps why specific compounds are "outliers" (i.e. predicted far outside the expected data range). Also 3D QSAR does not account for conformational changes which occur as the ligand binds to the transporter, which current biological studies suggest likely occur and that it is not the binding of the inhibitor, but rather the increased $K m$ of the translocation step which is key to inhibition. ${ }^{62}$ This implies that there will be limitations to 3D modeling, which is where the homology model can potentially improve success of novel inhibitor discovery and design.

Past molecular modeling studies have primarily looked at nucleoside analogues. (See Figure 2-1 for nucleoside position numbering.) They found that the ribose ring was favored over other sugars, the 3' position of the ribose ring or an isostere in which a 


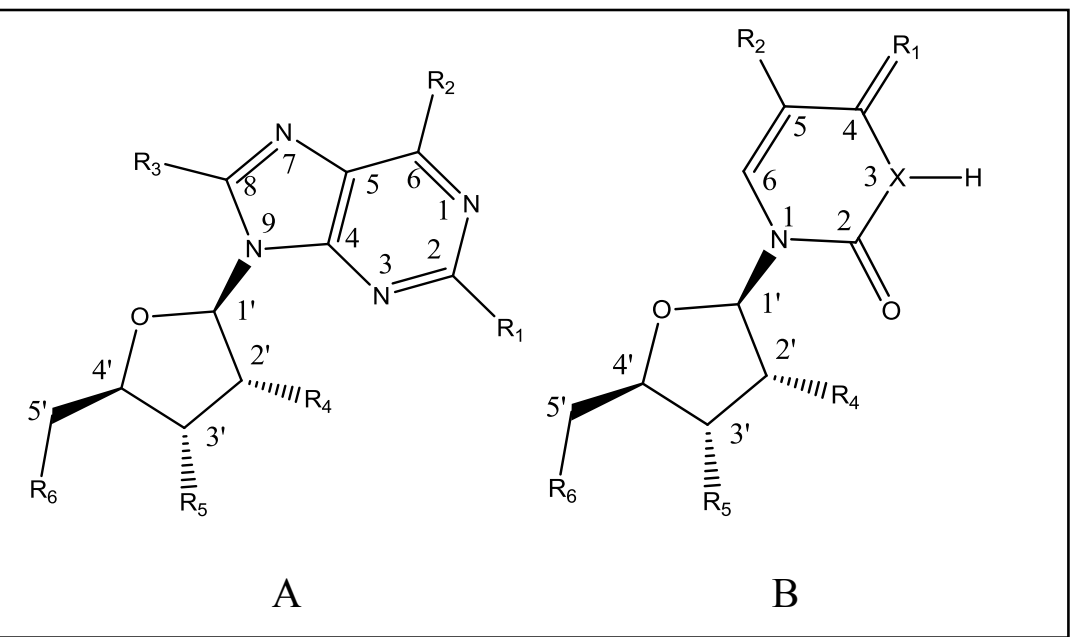

Figure 2-1. Nucleoside numbering on (A) purine and (B) pyrimidine 
hydrogen bond donor and accepting group occupied the same space was required for hENT1 activity. Similarly both hCNT1 and hCNT2 required the H-bond acceptor at 3'C and at the 2 position of the pyrimidine ring, but only hCNT2 seemed to require a H-bond acceptor at 5'. hCNT2 seemed to involve primarily H-bond interactions while hCNT1 utilized electrostatic and steric interactions as well. ${ }^{63}$ Two studies ${ }^{64,65}$ described 3D QSAR results of NBMPR analogues and their interaction with hENT1 which not only agreed with prior research, but correlated with an NMR study ${ }^{66}$ which demonstrated an anti-configuration rather than the syn configuration seen in X-ray diffraction and $\mathrm{X}$-ray crystals of NBMPR. ${ }^{67,68}$

Ho et al did a study on hENT4 in which they looked at substrate and inhibitor structure-activity relationships at physiological $\mathrm{pH} .{ }^{69} \mathrm{~A}$ hydrophobic aryl group spaced 0.5 to $7.7 \AA$ appeared to be key, with $\mathrm{IC}_{50}$ s decreasing with increasing hydrophobicity. ${ }^{69}$ It is important to note that the $\mathrm{IC}_{50}$ range of the substrates and inhibitors tested ranged from roughly $9 \mu \mathrm{M}$ to upwards of $1000 \mu \mathrm{M}$ or more. In contrast, Wang et al., which looked at hENT4 activity under the acidic conditions one might find in ischemic conditions identified potent nanomolar dipyridamole derivatives. ${ }^{1}$ The QSAR studies for that biological set is presented in this work.

Another modeling study looked at benzopyranone derivatives and related compounds and their interaction with the concentrative nucleoside transporters. ${ }^{70}$ It identified the molecules used in the creation of the pharmacophore models that will be presented in this work. While the CoMFA and CoMSIA models presented highlighted the interactions necessary for potency within that scaffold, and can predict potency for similar molecules, it is less adept at identifying new scaffolds, which was the goal of the pharmacophore models.

Finally, Hu et al. examined hCNT3 with CoMFA and CoMSIA and determined that electrostatic interactions played the role in hCNT3 substrate affinity, with the 3 'hydroxyl being a key structural feature. ${ }^{71}$ In addition, they determined that steric hindrance at the 3-position and a positive charge at the 5-positition were favorable for hCNT3 transport. ${ }^{71}$ When they looked at pharmacophore features, the minimum required for substrate transport by hCNT3 was a hydrogen bond acceptor at 3'-OH and 5'-O in addition to a hydrophobic center in the position of the pyrimidine base ring. ${ }^{71}$

It is important to note that a number of prior studies focus on substrates or do not differentiate between substrates and inhibitors. Ideally, when designing an inhibitor it will retain affinity, binding tightly, but prove to be a poor substrate, thus inhibiting the transporter. This suggests that the pharmacophore for competitive inhibitors would encompass that of substrates, but contain another element(s). Once enough diverse, potent, and selective inhibitors are identified, that is an area that requires exploration.

\section{Experimental Methods}

Schrodinger's Maestro modeling suite was used to build a database of molecules. ${ }^{72}$ After which, the PHASE module was used to minimize, generate 
conformers, identify pharmacophore elements, and generate hypotheses using default settings. ${ }^{73}$ Hypotheses were scored by fit and selected based on the pharmacophore's $\mathrm{q}^{2}$ statistics. For data sets in which CoMFA and/or CoMSIA models were generated, the resulting hypothesis and molecular alignments were exported into Sybyl $\mathrm{X}^{74}$ where CoMFA and CoMSIA descriptors were generated and PLS models were established. CoMFA and CoMSIA models were validated by leave-one-out cross-validation, and external test sets. External test sets were chosen by ranking the data set according to activity. Then every eleventh compound, which equated to approximately ten percent of the database, was selected to comprise a representative selection of molecules of varying activity. For in silico screening, the pharmacophore hypothesis in PHASE was used to screen the University of Cincinnati's proprietary database of about 360,000 small molecule compounds. Compounds were initially cherry picked for novel structure (CNT3 model). One hundred and ninety-two compounds were selected for biological testing.

For the CNT1 model, a 500 molecular weight cut off was applied and hits were ranked according to predicted $-\log \mathrm{IC}_{50}$. Excluding compounds of flavonoid like structures or nucleoside structures, or molecules containing functional groups such as carboxylates which have never demonstrated high nucleoside transporter affinity, the top ninety-six compounds were selected for biological testing.

\section{Results}

\section{hENT1 Models}

In an attempt to explore the structure-activity relationship of benzopyranone (flavonoid) analogues with nucleoside transporters, biological data from the Wang et al. study was used to develop a pharmacophore hypothesis. ${ }^{70}$ The three most active compounds were used define "active" and three compounds with much higher $\mathrm{IC}_{50} \mathrm{~s}$ were used to define "inactive" (Figure 2-2).

The hypothesis (Figure 2-3) was used to align the database. By itself, it had poor predicative capability. Table 2-1 shows the CoMFA statistics. The flavonoid compounds were not particularly potent, and the compounds were of high similarity, neither of which is ideal for producing a robust model.

The CoMFA model indicates that both steric and electrostatic interactions play a role in the binding of the flavonoid ligands to the hENT1 transporter. Figure 2-4 shows the residuals plot, with the actual $-\log \mathrm{IC}_{50} \mathrm{~S}$ on the $\mathrm{x}$-axis and the predicted $-\log \mathrm{IC}_{50} \mathrm{~S}$ on the y-axis. The spread of the test set is a little wide, which suggests that the model is perhaps slightly over fit. Given the poor predictive capability of the pharmacophore and the CoMFA model and the inability to identify a statistically significant CoMSIA model, the flavonoids proved of little use in terms of identifying a useful pharmacophore. 


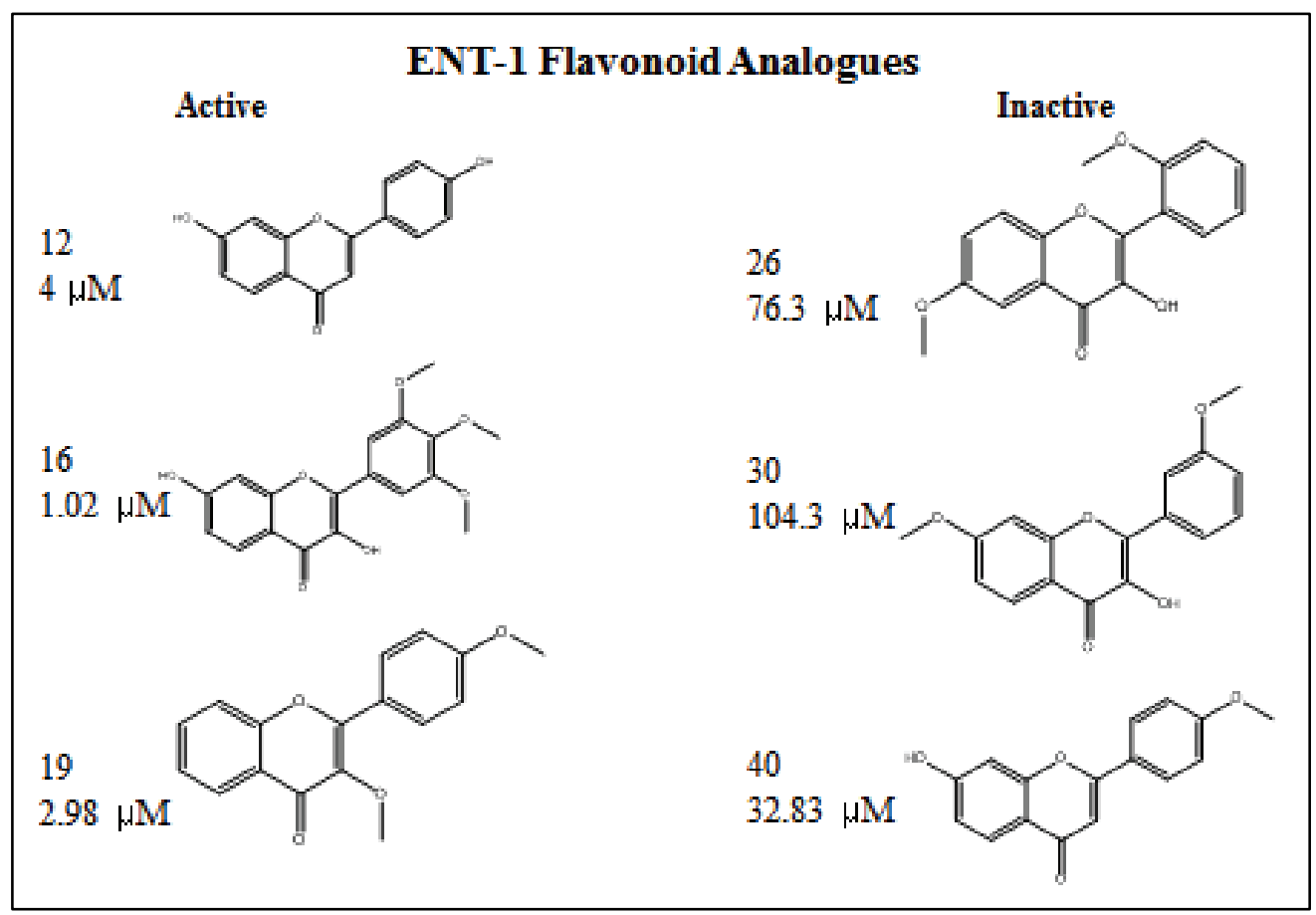

Figure 2-2. Flavonoid active and inactive sets used for the creation of ENT1 pharmacophore

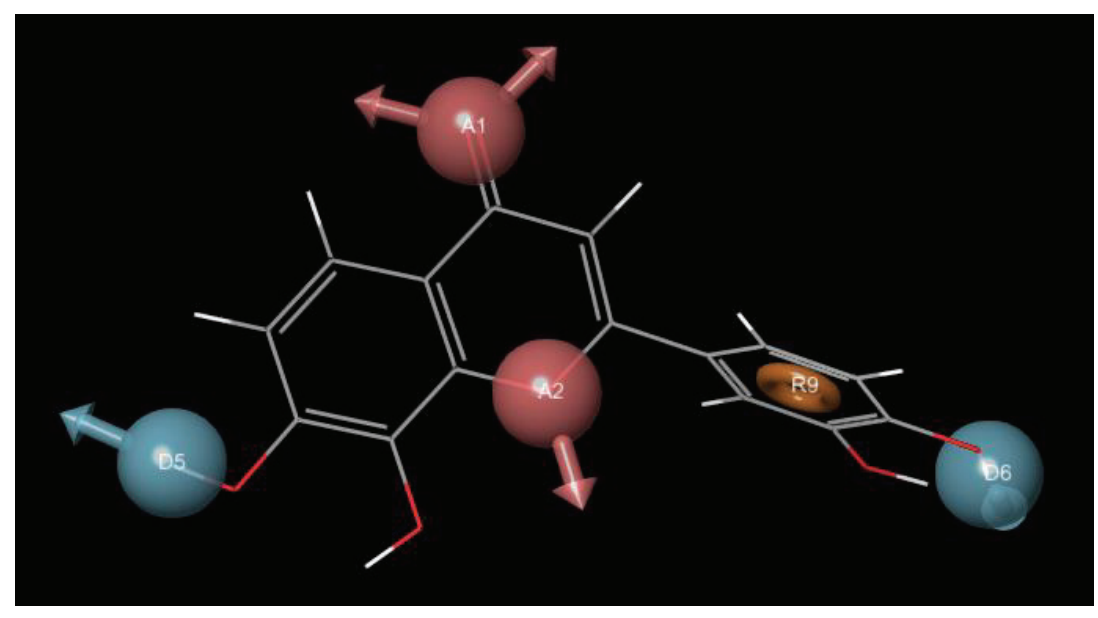

Figure 2-3. Flavonoid ENT1 5-point pharmacophore hypothesis

Note: Light blue spheres indicate hydrogen bond donor, red spheres are hydrogen bond acceptors, and orange rings are aromatic rings. 
Table 2-1. Flavonoid hENT1 CoMFA statistics

\begin{tabular}{lc} 
PLS Statistics & hENT1 CoMFA \\
\hline $\mathrm{q}^{2}\left(\mathrm{LOO}^{*}\right)$ & 0.460 \\
$\mathrm{r}^{2}$ & 0.888 \\
$\mathrm{~S}$ & 0.130 \\
$\mathrm{~F}$ & 61.544 \\
PLS components & 4 \\
No. of compounds used for model & 36 \\
No. of outliers & 6 \\
No. of compounds in external test set & 4 \\
Steric descriptor contribution & 0.519 \\
Electrostatic descriptor contribution & 0.481
\end{tabular}

Note: Leave one out (LOO)*

\section{ENT-1 PHASE Aligned CoMFA Analysis of Flavonoid Analogues}

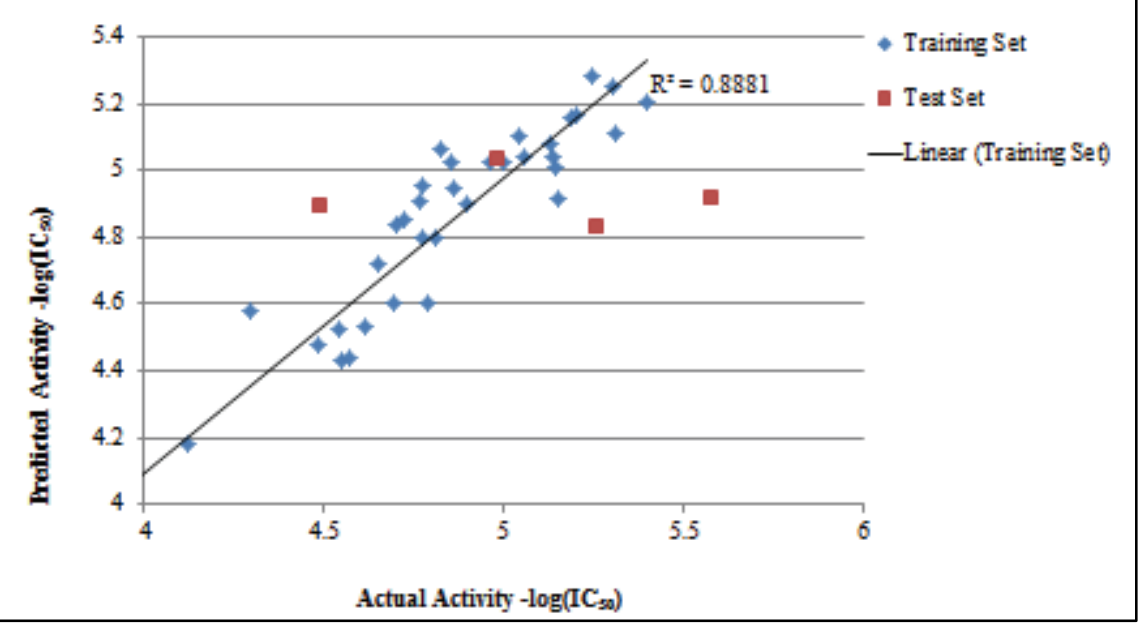

Figure 2-4. Predicted versus actual $-\log \mathrm{IC}_{50}$ of flavonoid analogues 
Similarly, models were created for both dipyridamole analogues and NBMPR analogues. Figure 2-5 and Figure 2-6 show the structures of the compounds used to generate the pharmacophore models.

In addition, a small series of new nucleoside analogues were synthesized (Chapter 3) and tested. Of the four active molecules the NBMPR model predicted two within one $\log$ unit, and three within $1.3 \log$ units. HCPN05 possessed activity over two $\log$ units lower than predicted (Figure 2-7), potentially due to lack of representation in the training set. Residual plots for the NBMPR models can be seen in Figure 2-8 and Figure 2-9.

Figure 2-10 illustrates the pharmacophores which were used to align the models. Both the nucleoside and dipyridamole models were statistically significant. While the NBMPR models resulted in higher $\mathrm{q}^{2} \mathrm{~s}$, they also had higher deviation about the regression line when compared to the dipyridamole models, suggesting that the NBMPR model is slightly over-fit. Comparing the PLS statistics in Table 2-2 it is of particular interest that both CoMSIA models arrive at nearly the same hydrophobic descriptor contribution, and similar hydrogen bond acceptor contributions, but differ greatly on the contribution of hydrogen bond donor interactions. Figure 2-11 and Figure 2-12 illustrate the compounds used to design the pharmacophore and Figure 2-13 shows the resulting pharmacophore. Given the results, the question then, was this a real difference, or an artifact of an over fit model?

Molecular studies in the literature indicate that while NBMPR and dipyridamole demonstrate competitive inhibition of each other, they do not likely bind to the exact same site on hENT1 ${ }^{75}$ Perhaps the difference in the hydrogen bond donor contributions accounted for this difference in binding. With this in mind, a pharmacophore model was produced using all of the compounds in the prior three studies, and that model was used to create CoMFA and CoMSIA models. As expected, the resulting model performed poorly in comparison to either of the NBMPR or dipyridamole models, reflecting that each of these models displayed statistically significant interactions that did not correlate with each other (Table 2-3). This also suggested that more than one binding location did in fact exist. The next step involved identifying which model had better predictive capability in identifying small molecule biologically active molecules.

While not quite the same as the dipyridamole model, it is interesting to note that that the hydrogen bond donor and acceptor contributions of the combined model compared more closely to that model than the NBMPR model.

To test the individual models, the structures of diverse compounds that had shown up as "hits" in a cellular screening assay, and for which $\mathrm{IC}_{50}$ data had been calculated, were put into a database. Since, flavonoids have a number of drawbacks, namely poor potency and poor biological selectivity. They hit a wide variety of biologically relative targets. In addition flavonoids have been widely examined and shown to have little room for chemical alterations. Thus, it was decided not to pursue them as leads. Given that the model did not perform as well, it was not included in the model comparison. 


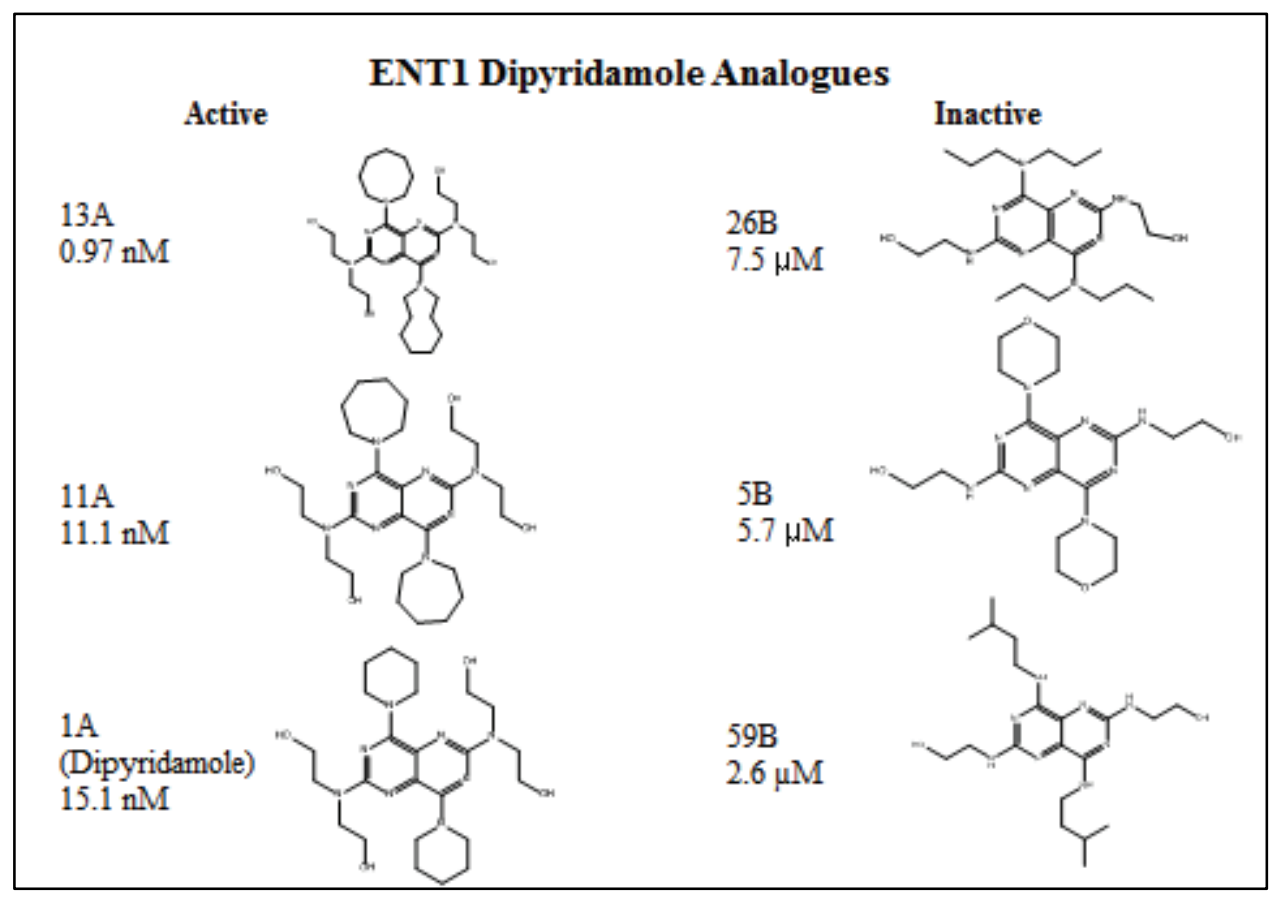

Figure 2-5. Dipyridamole analogues used for the creation of hENT1 pharmacophore

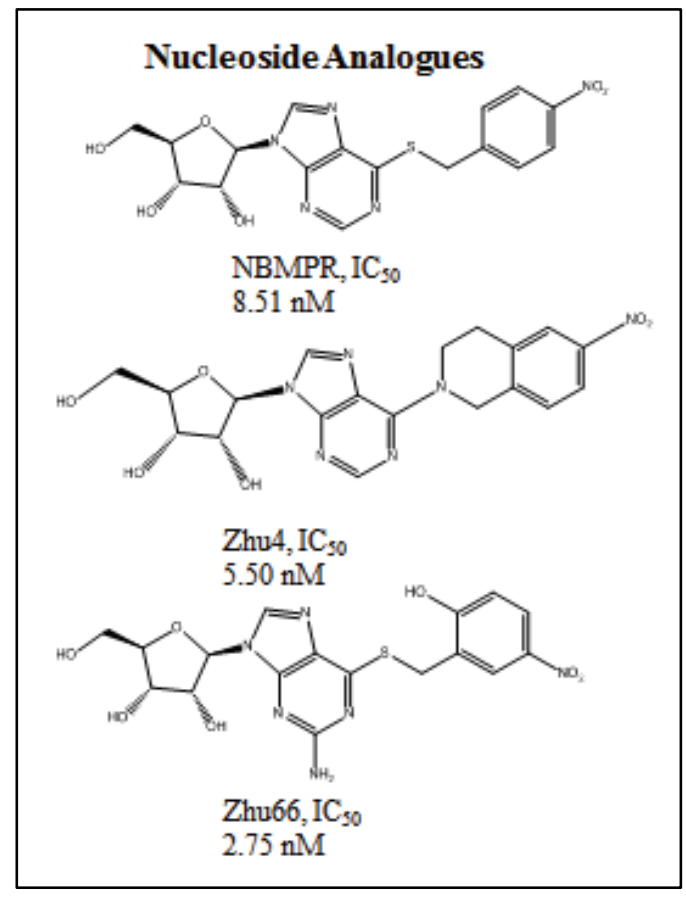

Figure 2-6. Active nucleoside analogues used for hENT1 pharmacophore 


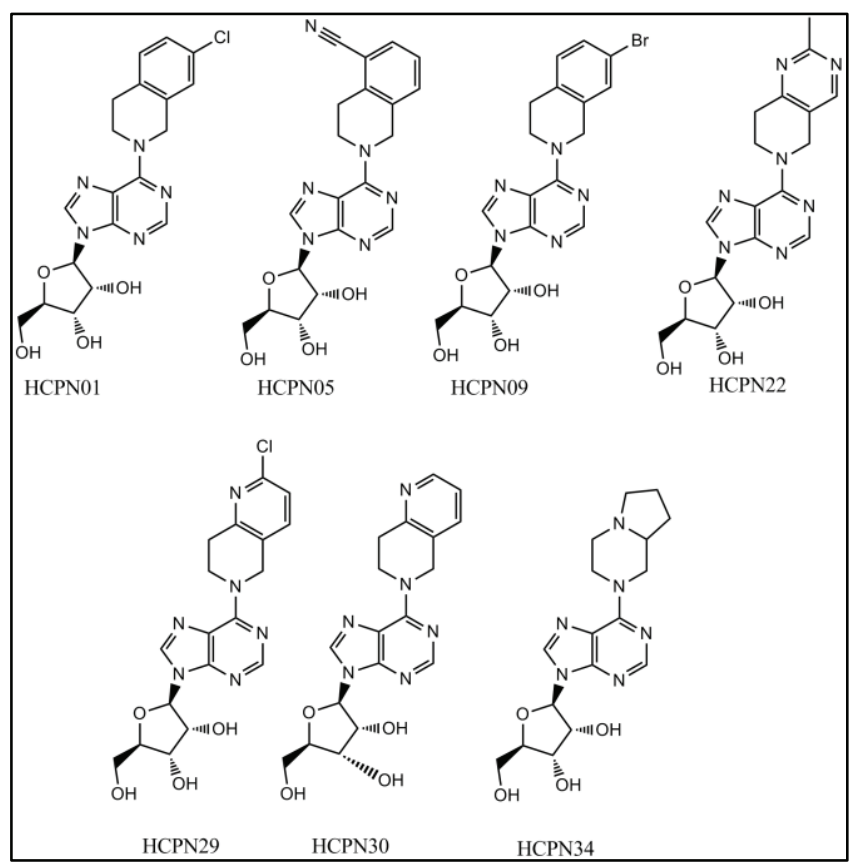

Figure 2-7. Synthesized nucleoside analogues

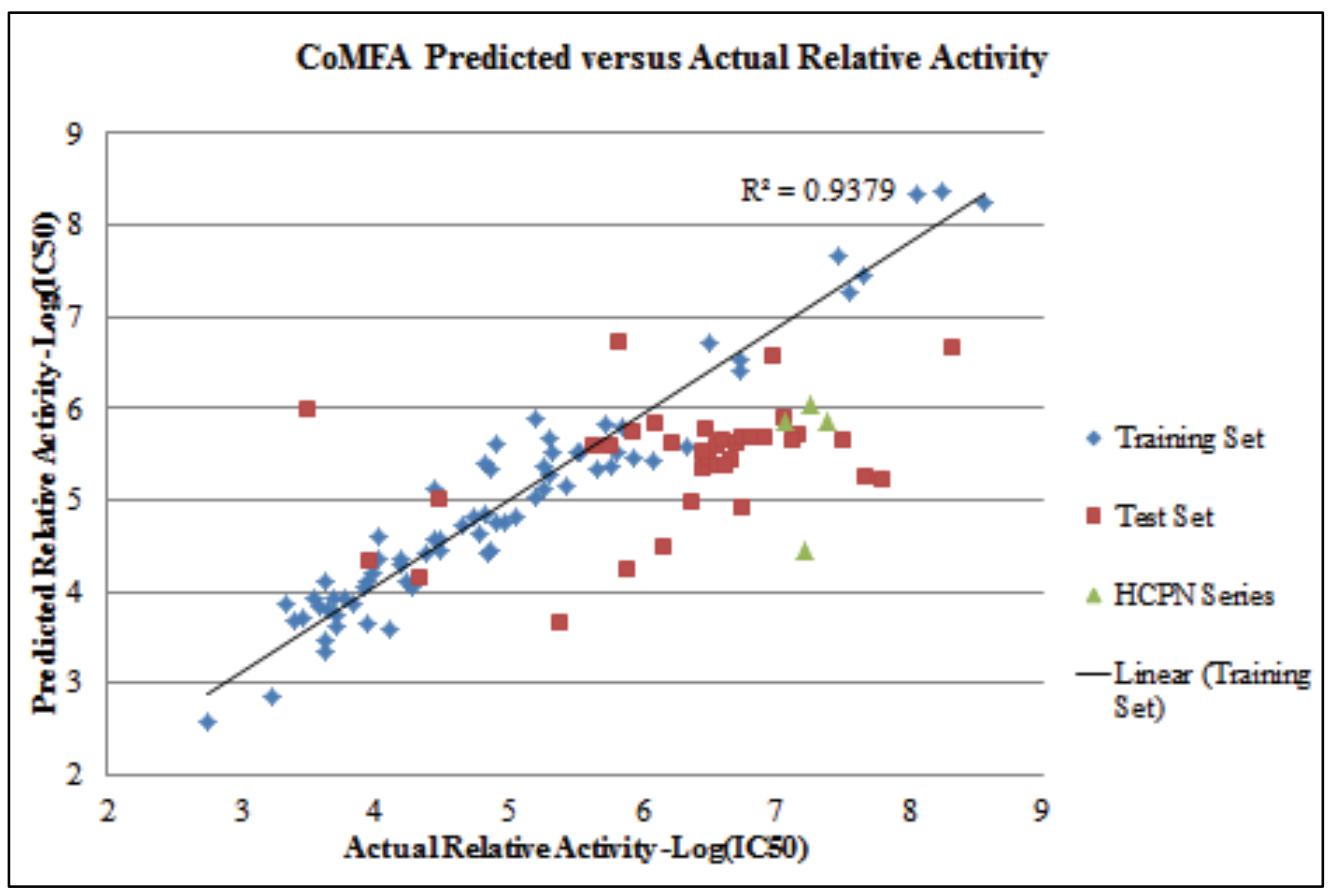

Figure 2-8. Prediction plot of hENT1 NBMPR CoMFA model 


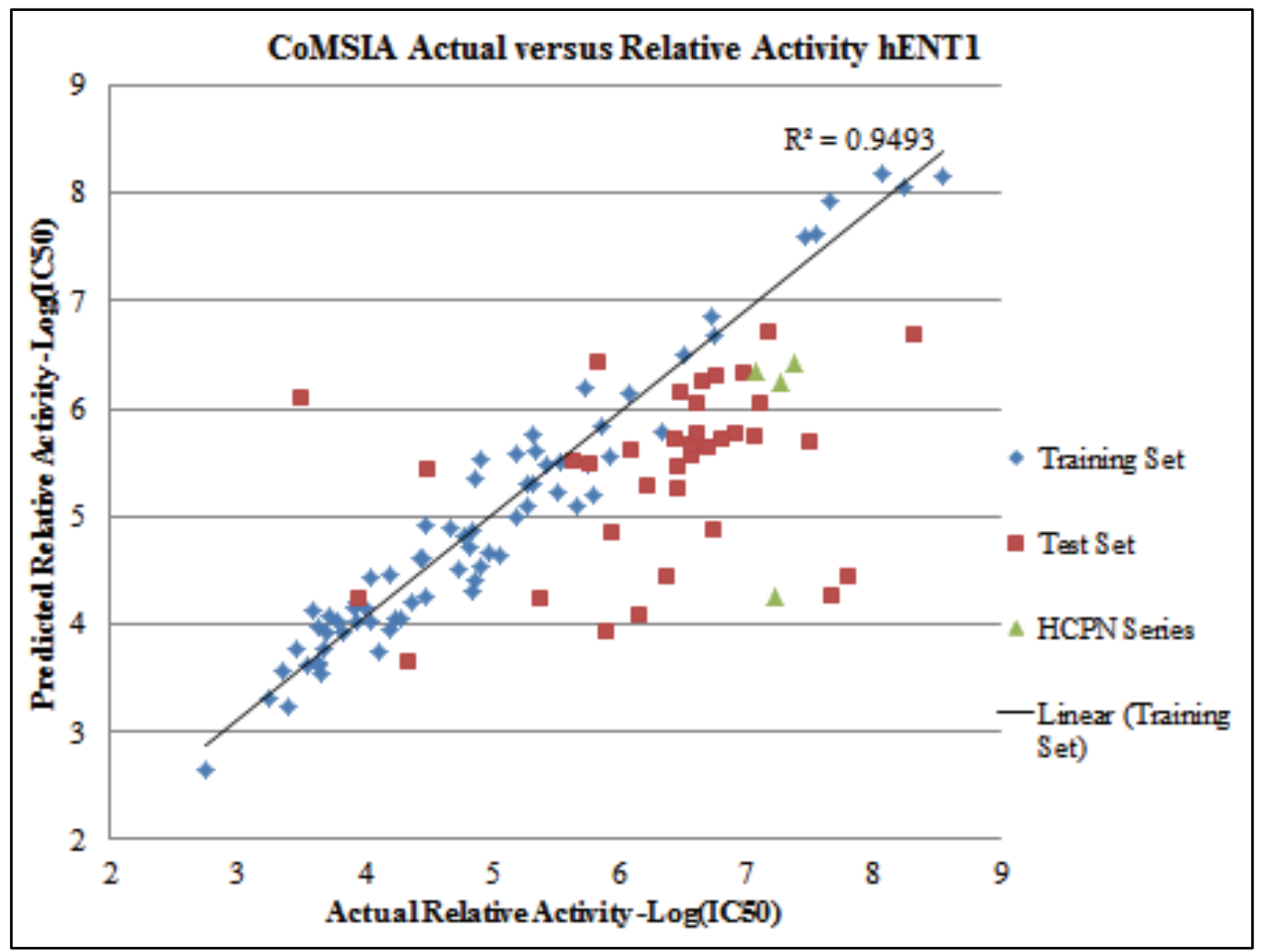

Figure 2-9. $\quad$ Prediction plot of hENT1 NBMPR CoMSIA model

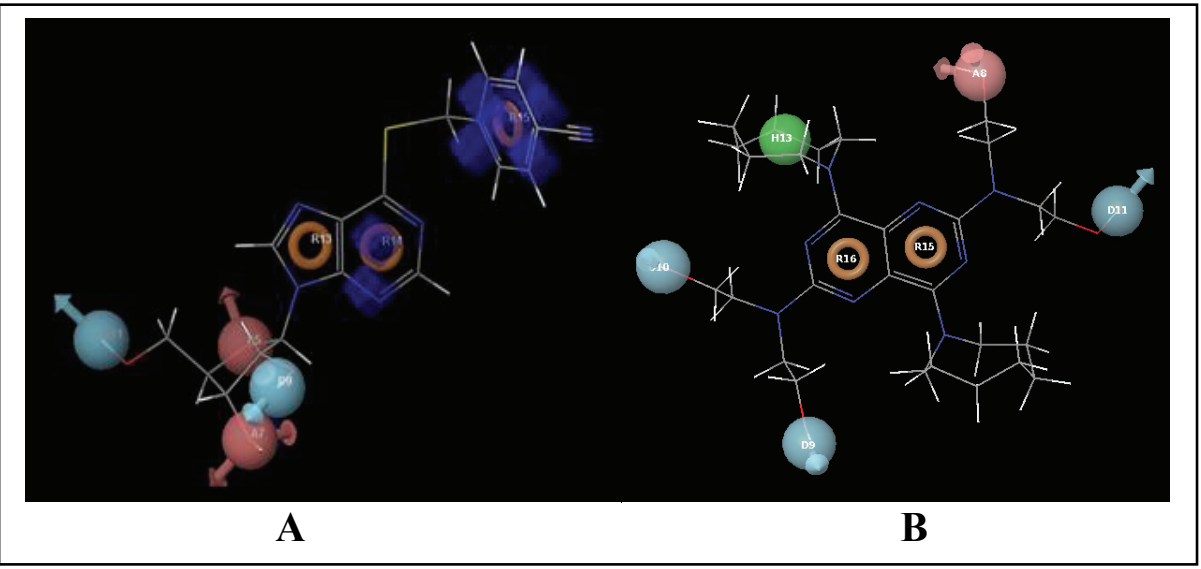

Figure 2-10. NBMPR (A) pharmacophore and dipyridamole (B) pharmacophore

Note: Light blue spheres indicate hydrogen bond donor, red spheres are hydrogen bond acceptors, orange rings are aromatic rings, and blue squares indicate favorable interactions for high potency in regards to the ligand's fit and the hypothesis. Blue boxes were emitted in (B) as their quantity obscured the pharmacophore points. 
Table 2-2. Dipyridamole and NBMPR analogue pharmacophore aligned models

\begin{tabular}{lcccc}
\hline PLS Statistics & $\begin{array}{c}\text { Dipyridamole } \\
\text { CoMFA }\end{array}$ & $\begin{array}{c}\text { Dipyridamole } \\
\text { CoMSIA }\end{array}$ & $\begin{array}{c}\text { NBMPR } \\
\text { CoMFA }\end{array}$ & $\begin{array}{c}\text { NBMPR } \\
\text { CoMSIA }\end{array}$ \\
\hline $\mathrm{q}^{2}$ (LOO) & 0.479 & 0.442 & 0.525 & 0.573 \\
$\mathrm{r}^{2}$ & 0.975 & 0.949 & 0.938 & 0.949 \\
$\mathrm{~S}$ & 0.107 & 0.152 & 0.334 & 0.302 \\
$\mathrm{~F}$ & 249.576 & 120.637 & 161.053 & 199.655 \\
$\begin{array}{l}\text { PLS components } \\
\text { Number of compounds used } \\
\text { for model }\end{array}$ & 6 & 6 & 6 & 6 \\
$\begin{array}{l}\text { Number of outliers } \\
\begin{array}{l}\text { Number of compounds in } \\
\text { external test set }\end{array}\end{array}$ & 46 & 46 & 70 & 70 \\
$\begin{array}{l}\text { Steric descriptor contribution } \\
\begin{array}{l}\text { Electrostatic descriptor } \\
\text { contribution }\end{array}\end{array}$ & 0.398 & 0.077 & 0.510 & 0.132 \\
$\begin{array}{l}\text { Hydrophobic descriptor } \\
\text { contribution }\end{array}$ & 0.602 & 0.213 & 0.490 & 0.360 \\
$\begin{array}{l}\text { H-bond donor descriptor } \\
\text { contribution }\end{array}$ & N/A & 0.287 & N/A & 0.286 \\
$\begin{array}{l}\text { H-bond acceptor descriptor } \\
\text { contribution }\end{array}$ & N/A & 0.289 & N/A & 0.098 \\
\hline & N/A & 0.134 & N/A & 0.124 \\
\hline
\end{tabular}


<smiles>OCCN(CCO)c1nc(N2CCCCC2)c2nc(N(CCO)CCO)nc(N3CCCCC3)c2n1</smiles>

$1 \mathrm{~A}-\mathrm{IC}_{50} 15.1 \mathrm{nM}$

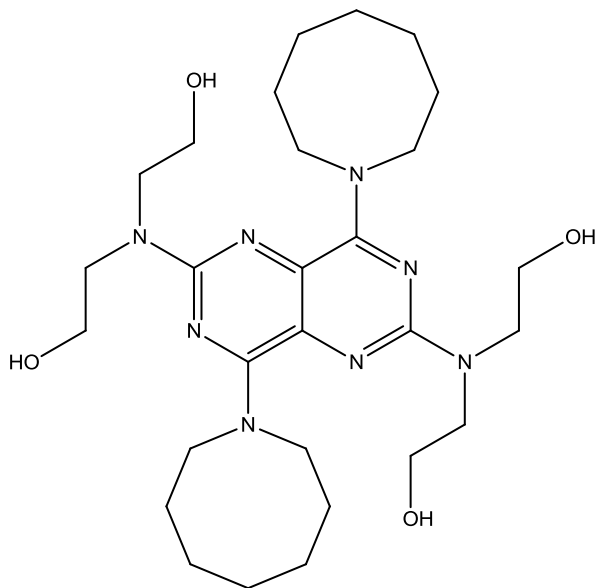

$13 \mathrm{~A}-\mathrm{IC}_{50} 0.97 \mathrm{nM}$<smiles>CC(=O)OCCN(CCOC(C)=O)c1nc(N2CCCCC2)c2nc(N3CCCCC3)nc(N(CCOC(C)=O)CCOC(C)=O)c2n1</smiles>

74- $\mathrm{IC}_{50} 69.2 \mathrm{nM}$

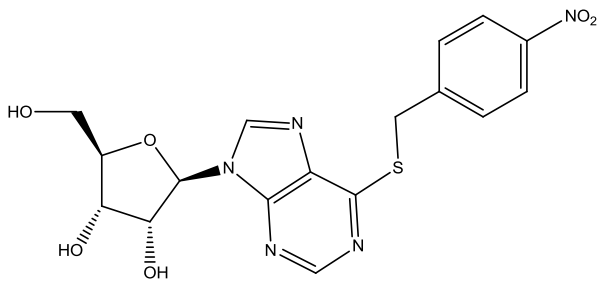

NBMPR- $\mathrm{IC}_{50} 8.51 \mathrm{nM}$

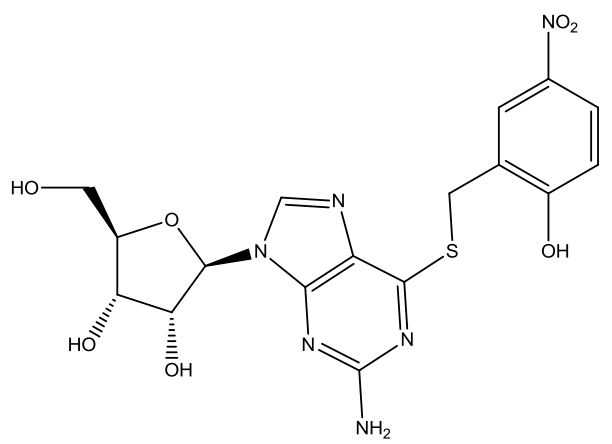

NBMPR66- $\mathrm{IC}_{50} 2.75 \mathrm{nM}$

Figure 2-11. Active set for ENT1 “combined" pharmacophore 
<smiles>COc1ccc(C(=O)/C=C/c2ccc(OC)cc2O)cc1</smiles>

Flavonoid45- $\mathrm{IC}_{50} 101.9 \mu \mathrm{M}$<smiles>COc1cc2ncc(-c3ccccc3)nc2cc1OC</smiles>

Flavonoid100- $\mathrm{IC}_{50} 0.13 \mu \mathrm{M}$<smiles>Cc1c(CNc2nc(N)nc3c2ncn3[C@@H]2O[C@H](CONc3cccc4c3CCN(c3ncnc5c3nc3n5[C@@H]5C[C@H](CCO5)[C@@H](O)[C@H]3O)C4)[C@@H](O)[C@H]2O)cccc1[N+](=O)[O-]</smiles>

NBMPR6- $\mathrm{IC}_{50} 7,413 \mu \mathrm{M}$<smiles>N#CC1=C(N)OC2c3ccccc3OC(=O)C2C1C1CC=CCC1</smiles>

Compound292- $\mathrm{IC}_{50} 30.0 \mu \mathrm{M}$

Figure 2-12. Inactive set for hENT1 "combined" pharmacophore 


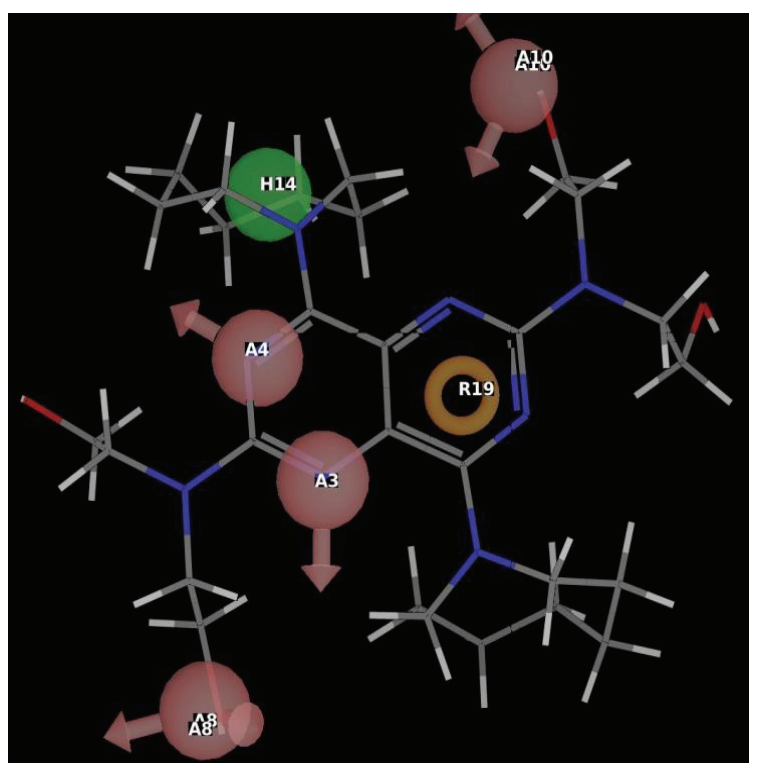

Figure 2-13. "Combined" pharmacophore hypothesis with active compound 13A overlaid

Note: Light blue spheres indicate hydrogen bond donor, red spheres are hydrogen bond acceptors, orange rings are aromatic rings, and the green sphere represents a hydrophobic interaction. 
Table 2-3. "Combined" pharmacophore CoMFA and CoMSIA PLS statistics

\begin{tabular}{lcc}
\hline PLS Statistics & CoMFA & CoMSIA \\
\hline $\mathrm{q}^{2}$ (LOO) & 0.474 & 0.482 \\
$\mathrm{r}^{2}$ & 0.687 & 0.852 \\
$\mathrm{~S}$ & 0.654 & 0.456 \\
$\mathrm{~F}$ & 160.67 & 207.81 \\
PLS components & 3 & 6 \\
Number of compounds used for model & 224 & 223 \\
Number of outliers & 4 & 5 \\
Number of compounds in external test set & 24 & 24 \\
Steric descriptor contribution & 0.533 & 0.140 \\
Electrostatic descriptor contribution & 0.467 & 0.303 \\
Hydrophobic descriptor contribution & $\mathrm{n} / \mathrm{a}$ & 0.186 \\
H-bond donor descriptor contribution & $\mathrm{n} / \mathrm{a}$ & 0.150 \\
H-bond acceptor descriptor contribution & $\mathrm{n} / \mathrm{a}$ & 0.220 \\
\hline
\end{tabular}


As such the NBMPR and dipyridamole models were tested in their ability to pick active compounds from the database of known active molecules. The PHASE application was used to calculate conformations of the compounds in the database and tested them for fit in the pharmacophore. Neither pharmacophore could do so utilizing all seven points. However, when relaxed to four points, the dipyridamole model could identify all but one of the compounds and at three points, identified all of them. Even at three points, the NBMPR model could not identify the molecules. Table 2-4 shows the predicted and actual $-\log \mathrm{IC}_{50} \mathrm{~s}$ of the compounds using the dipyridamole model.

So while the NBMPR model identifies interactions important for binding of nucleosides and their analogues, it does not accurately reflect the interactions of small molecules. The bare minimum of three points required for the dipyridamole pharmacophore to identify hits, likely points to the key interactions required for affinity, while the other points potentially add to potency. Since, none of the small molecule hits were as potent as either dipyridamole or NBMPR, logic suggests that utilizing the pharmacophore to design analogues with functional groups that can occupy the three dimensional space indicated in the pharmacophore could lead to more potent compounds.

\section{hENT4}

Similar to hENT1, the flavonoid data were used to build pharmacophore, CoMFA and CoMSIA models (Figure 2-14 and Table 2-5). In addition the same model types were generated from dipyridmole analogue previously published biological data, ${ }^{1}$ of which the CoMFA and CoMSIA statistics are presented in Table 2-6. In addition, the residual plots (Figure 2-15 and Figure 2-16) depict the predictive ability of the models. The CoMFA model clearly demonstrated better predictive capability in regards to the test set than the CoMSIA model.

As previously discussed, the flavonoids were not pursued for further study. Rather focus shifted to understanding the structure-activity relationship of the dipyridamole analogues via CoMFA and CoMSIA models. Even so, comparing the two models proves interesting. Both data sets produce models with very similar descriptor contributions, especially for the CoMSIA models. One could infer that the flavonoids potentially bind similarly to the dipyridamole analogues.

\section{hCNT3}

Using biological data from Wang et al. ${ }^{70}$ a pharmacophore model was generated for hCNT3. The study also published CoMFA and CoMSIA analysis, but while both methods are good at interpolating predicted activities of similar molecules, they do not do well at predicting the activity of novel molecular scaffolds. As mentioned before, it was desired to fine active compounds outside of the flavone chemical class. The pharmacophore model, which examined multiple conformations and does not rely on an alignment, served as a valuable tool for identifying new potentially active chemical classes. Figure 2-17 pictures the model chosen for the hCNT3 in silico screening. As 
Table 2-4. PLS statistics for hENT4 flavonoid pharmacophore-aligned models

\begin{tabular}{lcc}
\hline PLS Statistics & CoMFA & CoMSIA \\
\hline $\mathrm{q}^{2}$ (LOO) & 0.514 & 0.483 \\
$\mathrm{r}^{2}$ & 0.835 & 0.970 \\
$\mathrm{~S}$ & 0.214 & 0.097 \\
$\mathrm{~F}$ & 54.147 & 152.357 \\
PLS components & 3 & 6 \\
No. of compounds used for model & 36 & 35 \\
No. of Outliers & 4 & 5 \\
No. of compounds in external test set & 4 & 4 \\
Steric descriptor contribution & 0.610 & 0.105 \\
Electrostatic descriptor contribution & 0.390 & 0.295 \\
Hydrophobic descriptor contribution & N/A & 0.189 \\
H-bond donor descriptor contribution & N/A & 0.287 \\
H-bond acceptor descriptor contribution & N/A & 0.133 \\
\hline
\end{tabular}




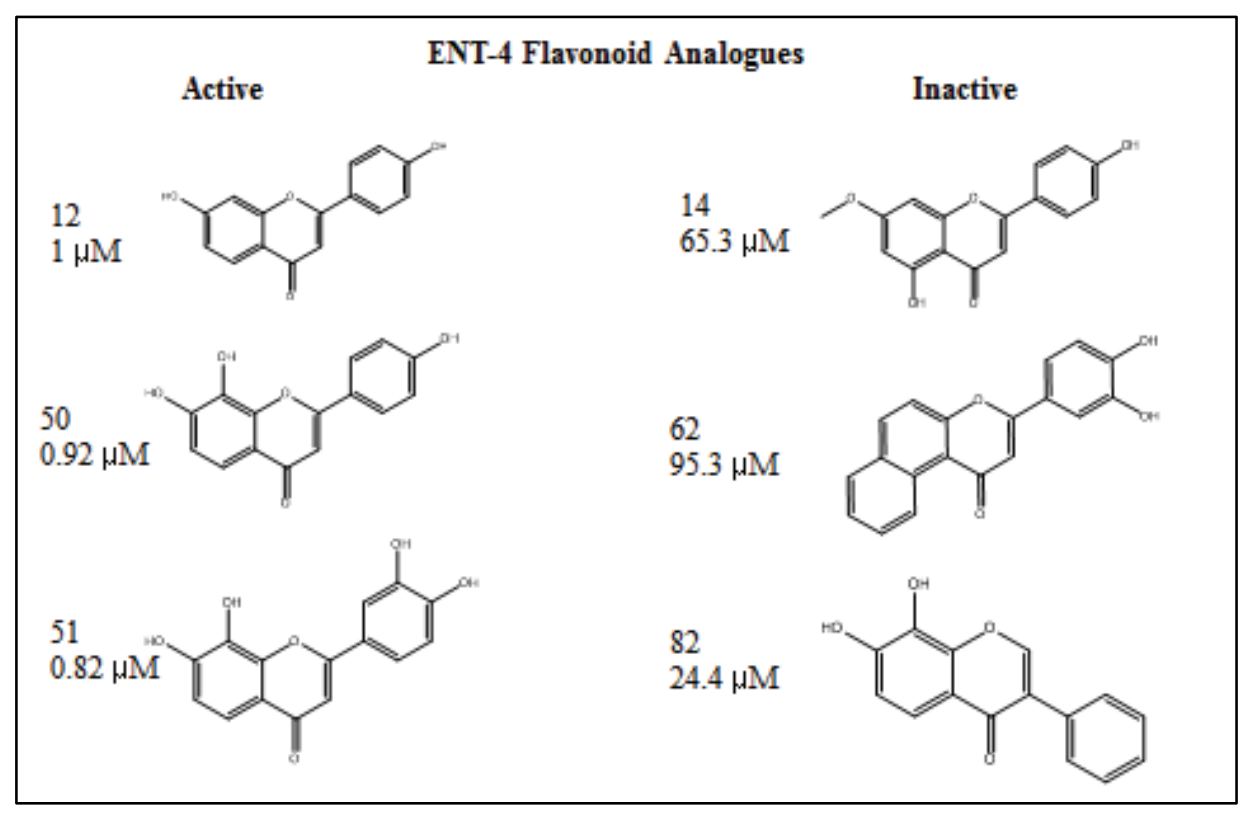

Figure 2-14. hENT4 flavonoid compounds used for pharmacophore design 
Table 2-5. PLS statistics for hENT4 dipyridamole pharmacophore-aligned models

\begin{tabular}{lcc}
\hline PLS Statistics & CoMFA & CoMSIA \\
\hline $\mathrm{q}^{2}(\mathrm{LOO})$ & 0.417 & 0.424 \\
$\mathrm{r}^{2}$ & 0.942 & 0.934 \\
$\mathrm{~S}$ & 0.216 & 0.233 \\
$\mathrm{~F}$ & 116.448 & 84.889 \\
PLS components & 5 & 3 \\
Number. of compounds used for model & 42 & 43 \\
Number of outliers & 8 & 8 \\
Number of compounds in external test set & 5 & 5 \\
Steric descriptor contribution & 0.607 & 0.117 \\
Electrostatic descriptor contribution & 0.393 & 0.191 \\
Hydrophobic descriptor contribution & N/A & 0.329 \\
H-bond donor descriptor contribution & N/A & 0.219 \\
H-bond acceptor descriptor contribution & N/A & 0.144 \\
\hline
\end{tabular}


Table 2-6. hCNT3 pharmacophore statistics

\begin{tabular}{lc}
\hline Statistic & Value \\
\hline \# Factors & 1 \\
$\mathrm{~S}$ & 0.3826 \\
$\mathrm{R}^{2}$ & 0.3316 \\
$\mathrm{~F}$ & 28.8 \\
$\mathrm{P}$ & $1.478 \times 10^{-6}$ \\
Stability & 0.4646 \\
$\mathrm{RMSE}$ & 0.2485 \\
$\mathrm{Q}^{2}$ & -1.0726 \\
Pearson-R & 0.6964 \\
\hline
\end{tabular}

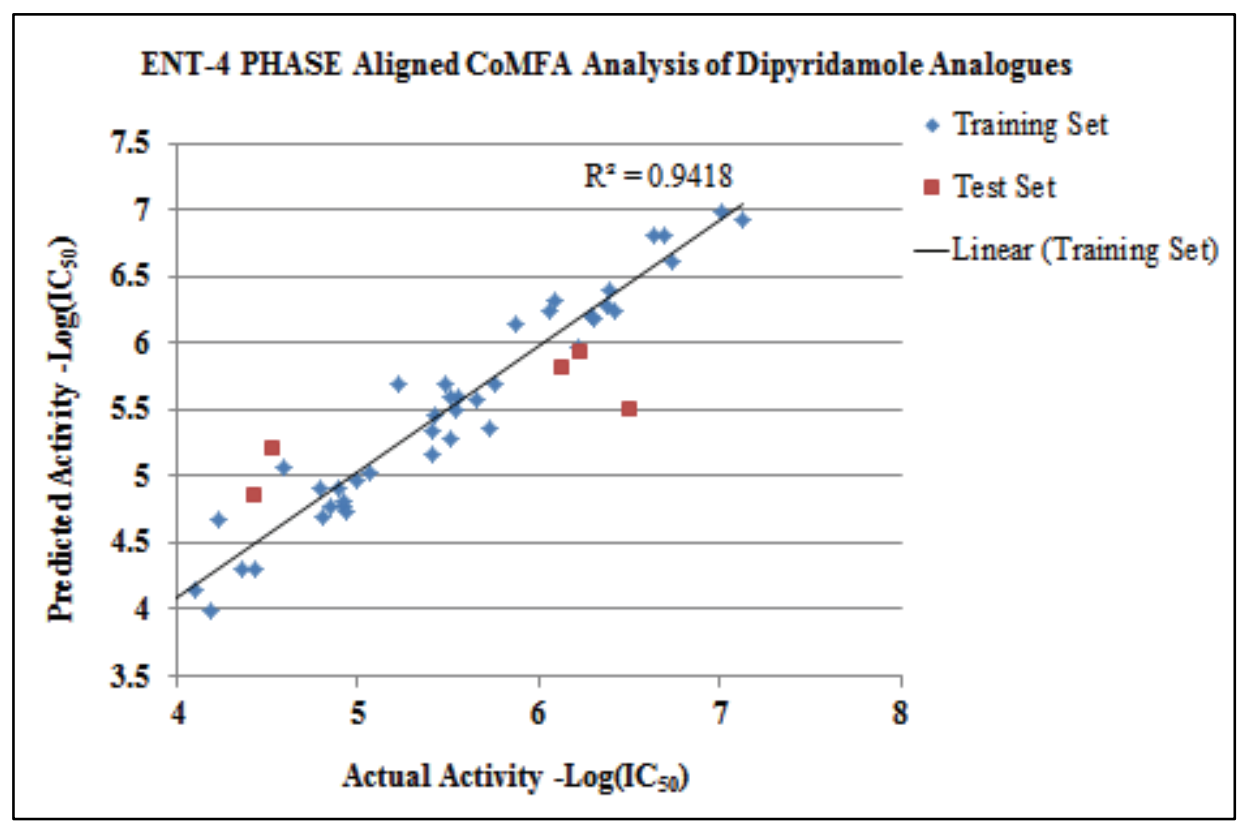

Figure 2-15. Residuals plot of CoMFA dipyridamole analogues 


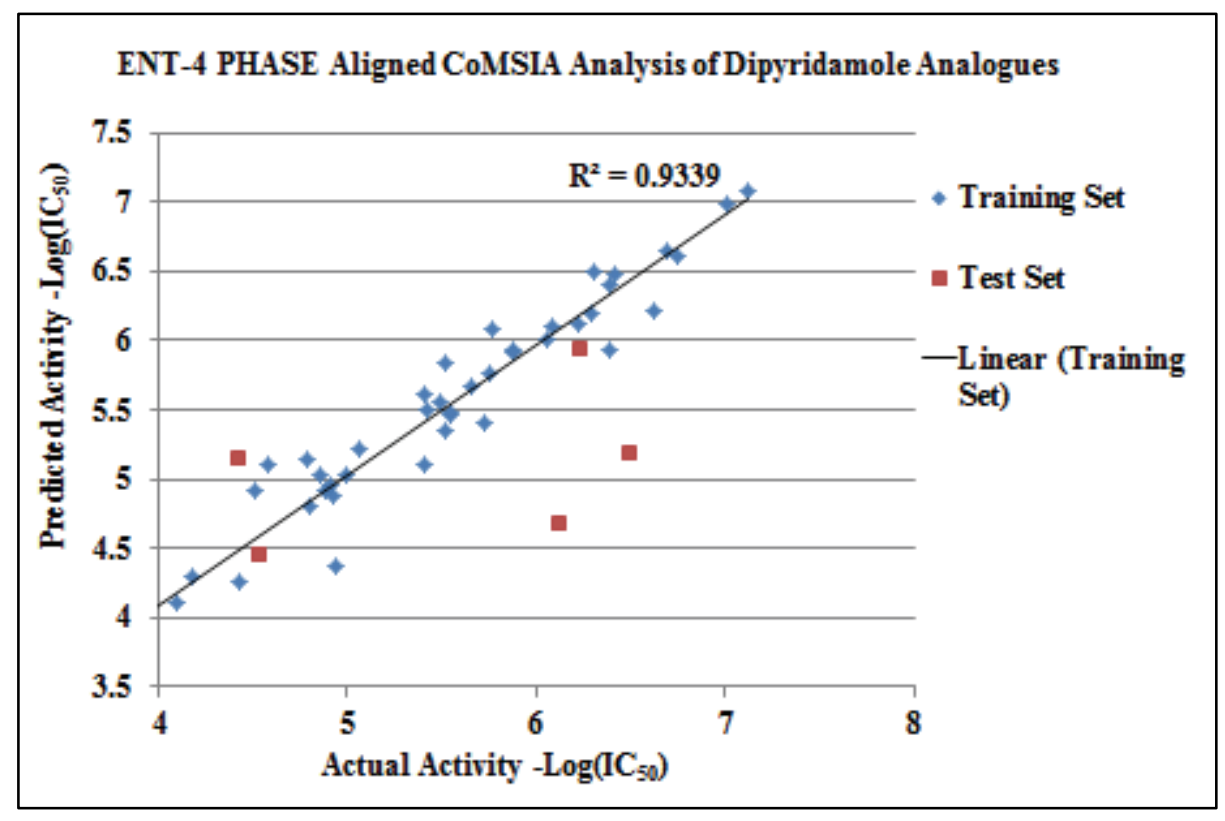

Figure 2-16. Residuals plot CoMSIA dipyridamole analogues

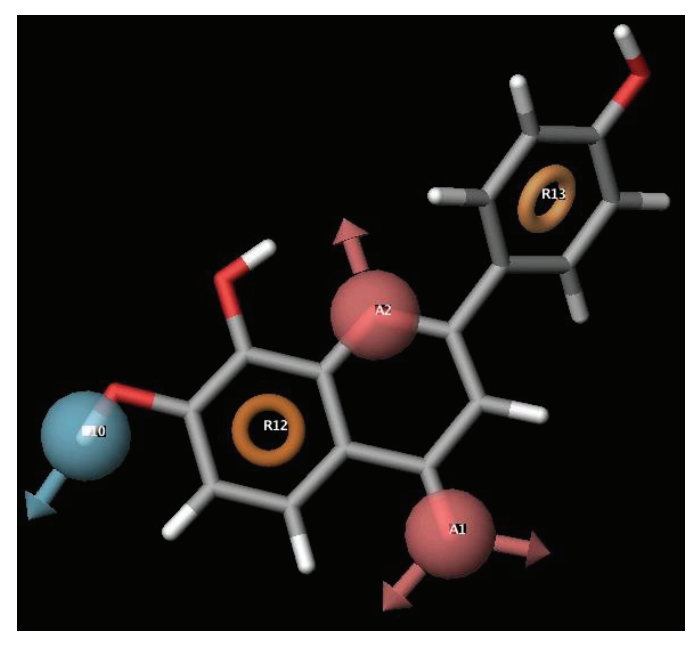

Figure 2-17. Flavone CNT3 pharmacophore

Note: Light blue spheres indicate hydrogen bond donor, red spheres are hydrogen bond acceptors, and orange rings are aromatic rings. 
biological screening was carried out at $10 \mu \mathrm{M}$, a cut off of $5.5\left[-\log \left(\mathrm{IC}_{50}\right)\right]$ was made, relegating much of the original data as "inactive". The top three most active molecules were used in design of the pharmacophore (Figure 2-18). Unfortunately, the model was not statistically significant (Table 2-7). However, it served as a starting point for screening.

The resulting pharmacophore was used to screen University of Cincinnati's proprietary database of 360,000 molecules. Of the hits, 192 compounds were cherrypicked for biological testing. Unfortunately, no significant hCNT3 hits were identified, but several hits were identified for the other transporters. (See Chapter 3 for screening results.)

\section{hCNT1}

Using the same published flavone data ${ }^{70}$, a pharmacophore was built for hCNT1 (Figure 2-19). The three most active and three least active compounds were used to generate the model. The resulting model was used to search the same database of compounds. A 500 molecular weight cutoff was applied and the hits ranked according to predicted activity. Any flavones or nucleoside derivatives were excluded and the top ninety-six available compounds were purchased for biological testing.

Two compounds demonstrated significant inhibition and their $\mathrm{IC}_{50}$ at $0.2 \mu \mathrm{M}[5-$ $\left.{ }^{3} \mathrm{H}\right]$ uridine were determined (see Chapter 3 for biological results). The original pharmacophore, due to a small initial active data set, had poor statistical significance. Using hit enrichment, i.e. adding the two hit compounds to the model, the new resulting model became significant, if still unstable. The resulting pharmacophore indicates a priority for hydrogen bond interactions rather than the aromatic rings seen in the original pharmacophore (Figure 2-20). This fits more in line with known substrates and the interactions necessary for nucleoside transport, but likely still is only a piece of the picture. Since the tested molecules showed a small degree of activity, it suggests that adding future analogue data to the model could further expand the SAR and improve the model (Table 2-8).

\section{Summary and Conclusions}

Several different computer models were created in an effort to assist in the discovery of novel scaffolds for small molecule inhibition of nucleoside transporters. Four different sets of 3D-QSAR models were developed for hENT1. Among these, the dipyridamole analogue based models appeared most relevant for identifying biologically active inhibitors of hENT1, although both the dipyidamole and NBMPR CoMFA and CoMSIA were statistically significant. While the NBMPR models illuminated binding interactions for nucleosides and nucleoside analogues, it did a poor job of identifying non-nucleoside inhibitors. Both CoMSIA models appeared consistent with hydrogen bond-acceptor and hydrophobic contributions, but the dipyridamole model suggested an 
<smiles>COc1cc2oc(-c3ccccc3)cc(=O)c2cc1O</smiles>

Flavone39

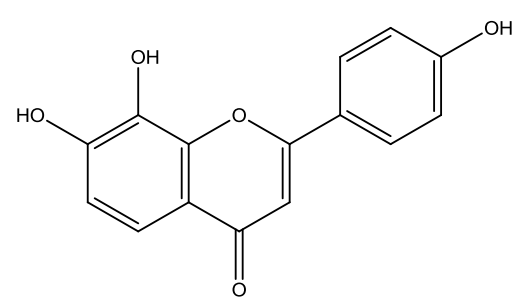

Flavone 50

$\mathrm{IC}_{50} 0.57 \pm 0.2 \mu \mathrm{M}$<smiles>O=c1c(O)c(-c2cc(O)c(O)c(O)c2)oc2cc(O)ccc12</smiles>

Flavone 84

$\mathrm{IC}_{50} 1.04 \pm 0.60 \mu \mathrm{M}$

Figure 2-18. Flavones used for hCNT3 pharmacophore design 
Table 2-7. hCNT1 flavone pharmacophore statistics for original and hit-enriched models

\begin{tabular}{lccccccccc}
\hline Model & \# Factors & SD & $\mathbf{R}^{2}$ & F & P & Stability & RMSE & $\mathbf{Q}^{\mathbf{2}}$ & $\begin{array}{c}\text { Pearson } \\
\mathbf{R}\end{array}$ \\
\hline Original & 1 & 0.2685 & 0.5958 & 22.1 & 0.000284 & 0.1235 & 0.1596 & -5.2444 & -0.6942 \\
Hit-enriched model & 1 & 0.2309 & 0.7011 & 35.2 & $2.76 \mathrm{E}-05$ & -0.3809 & 0.0402 & 0.6039 & 0.8708 \\
\hline
\end{tabular}

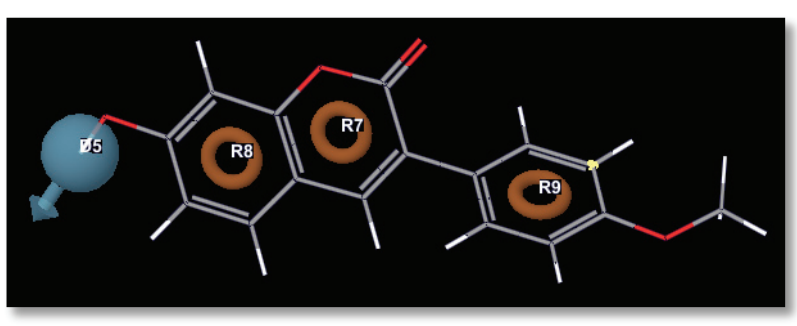

Figure 2-19. hCNT1 flavone pharmacophore 


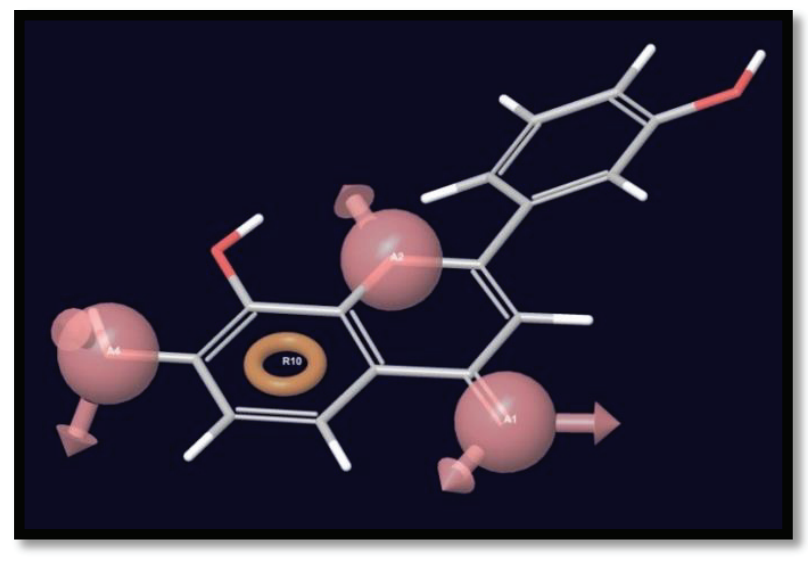

Figure 2-20. Hit enriched hCNT1 pharmacophore

Table 2-8. Dipyridamole pharmacophore search predictions

\begin{tabular}{lcc}
\hline ID & Predicted -Log Activity & Actual - Log Activity \\
\hline UC21 & 5.32 & 7.267606 \\
UC29* & 5.61 & 6.769551 \\
UC33* & 5.57 & 6.508638 \\
UC36* & 5.49 & 5.835647 \\
UC60 & 5.18 & 5.522879 \\
UC63 & $5.17-5.29 * *$ & 5.471083 \\
UC65 & 5.70 & 6.161151 \\
UC70 & 5.37 & 6.853872 \\
UC86 & $5.27-5.29 * *$ & 6.124939 \\
UC136 & $5.32-5.54 * *$ & 5.595166 \\
UC137 & $5.18-5.32^{* *}$ & 5.278189 \\
UC148 & $5.62-5.67$ & 6.107905 \\
UC173 & 5.27 & 5.730487 \\
UC175 & 5.19 & 6.124939 \\
\hline
\end{tabular}

Note: *Compound appeared to have mixed activity. **Multiple ionization states were possible and scored separately. 
increased contribution from hydrogen bond donors and a decrease in both steric and electrostatic contributions (Table 2-2). Considering NBMPR possesses a nitro group, and dipyridamole and its potent analogues lack such a highly polar and strong hydrogen bond acceptor group, this prediction correlates with the chemical functionality. As the position of the nitro substituent greatly effected potency ${ }^{64}$ this suggested that steric interaction played a more important role in NBMPR analogue potency compared to the dipyridamole analogues, suggesting that this portion of the molecules may occupy the non-overlapping regions of the binding sites for hENT1.

In addition, for identifying non-nucleoside molecules in future studies, the dipyridamole pharmacophore demonstrated the ability to identify active molecules as opposed to the NBMPR pharmacophore. Neither pharmacophore could identify known hits in a database when using all seven pharmacophore points, nor with six points matched. However, at five points the dipyridamole model identified UC29 and UC65 as hits and when search criteria were relaxed to four points, all hits were identified. However, their predictive values do not show a quantitative trend. Even at four points, the NBMPR model could not identify any of the UC compound hits. Table 2-9 contains the structures of the UC hENT1 hits.

Without a transporter structure, one can only hypothesize in regards to binding, but the evidence suggests that small molecules bind more like dipyridamole than nucleoside analogues. Given that NBMPR and dipyridamole bind at overlapping sites, it is possible that other novel binding pockets exist, and that the small molecules may not all bind in the same manner. By its ability to select a wide variety of active molecules where the NBMPR model failed, the dipyridamole model appeared to support the theory of more than one binding location. When overlaying the compounds with the pharmacophore, not all hits occupied the same four pharmacophore points, further suggesting multiple binding modes.

In order to further explore this idea, a series of compound derivatives would have to be made and tested, and common molecular scaffold pharmacophore-aligned CoMFA and CoMSIA models compared with combined models. As was done with the "combined" hENT1 models, if the compounds bind in the same binding pocket, the inclusive models would demonstrate better predictive ability and statistics than the individual models.

Similarly, the 3D-QSAR models of hENT4, all of which were non-nucleoside based, correlated. This strongly suggested that the models identified the critical interactions required for potent inhibition of hENT4. In comparing the CoMSIA descriptors of the hENT1 dipyridamole model and the hENT4 model, it was noted that the distribution was very similar. However, hENT4 demonstrated a higher contribution of electrostatic interactions and less steric interactions in comparison to hENT1. Considering that hENT4 utilizes a proton, and at physiological $\mathrm{pH}$ transports primarily mono-amines, ${ }^{76}$ the shift in descriptor distribution correlated to known functionality, further supporting the validity of the models. 
Table 2-9. hENT1 hit properties

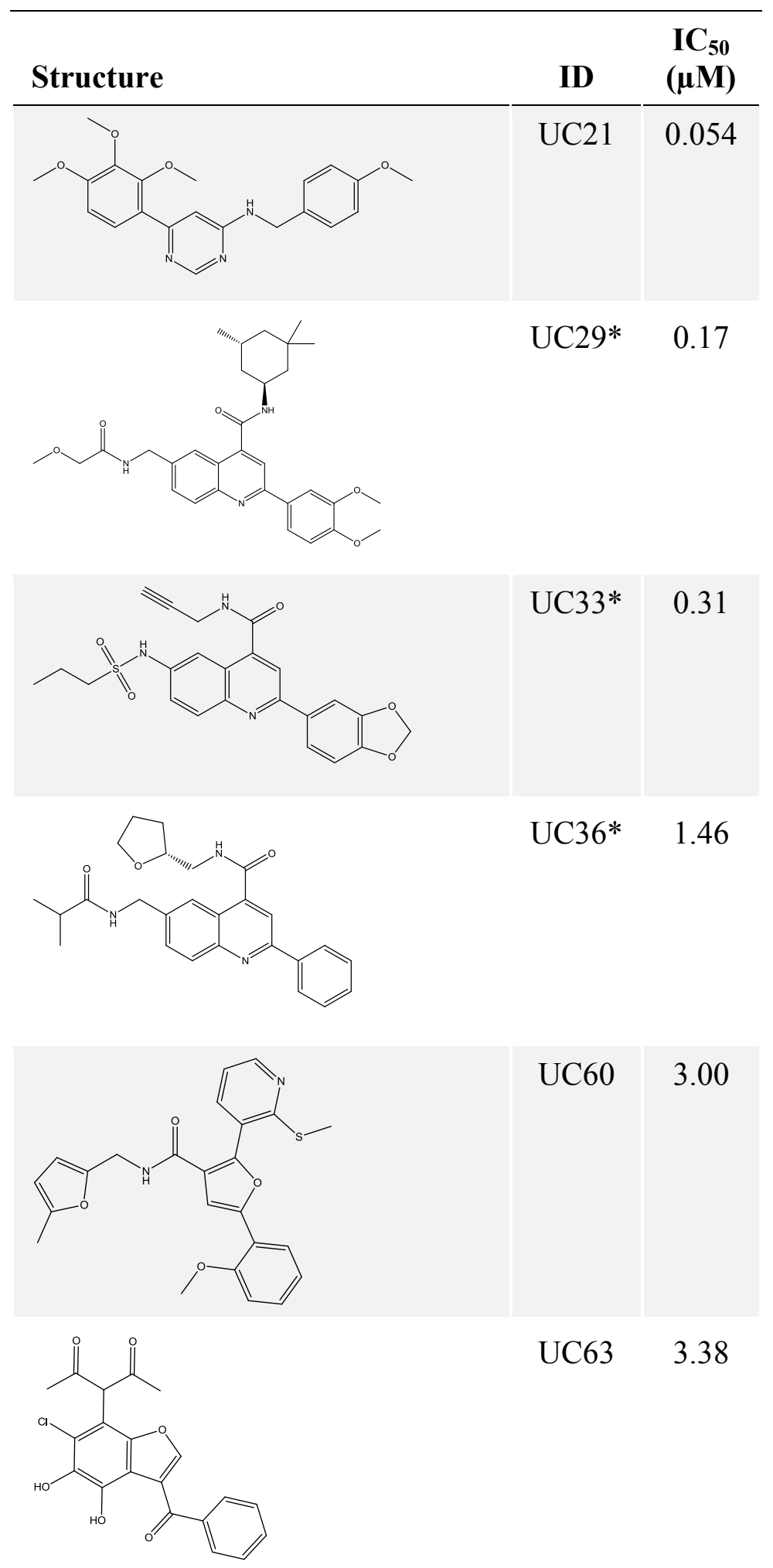


Table 2-9. Continued
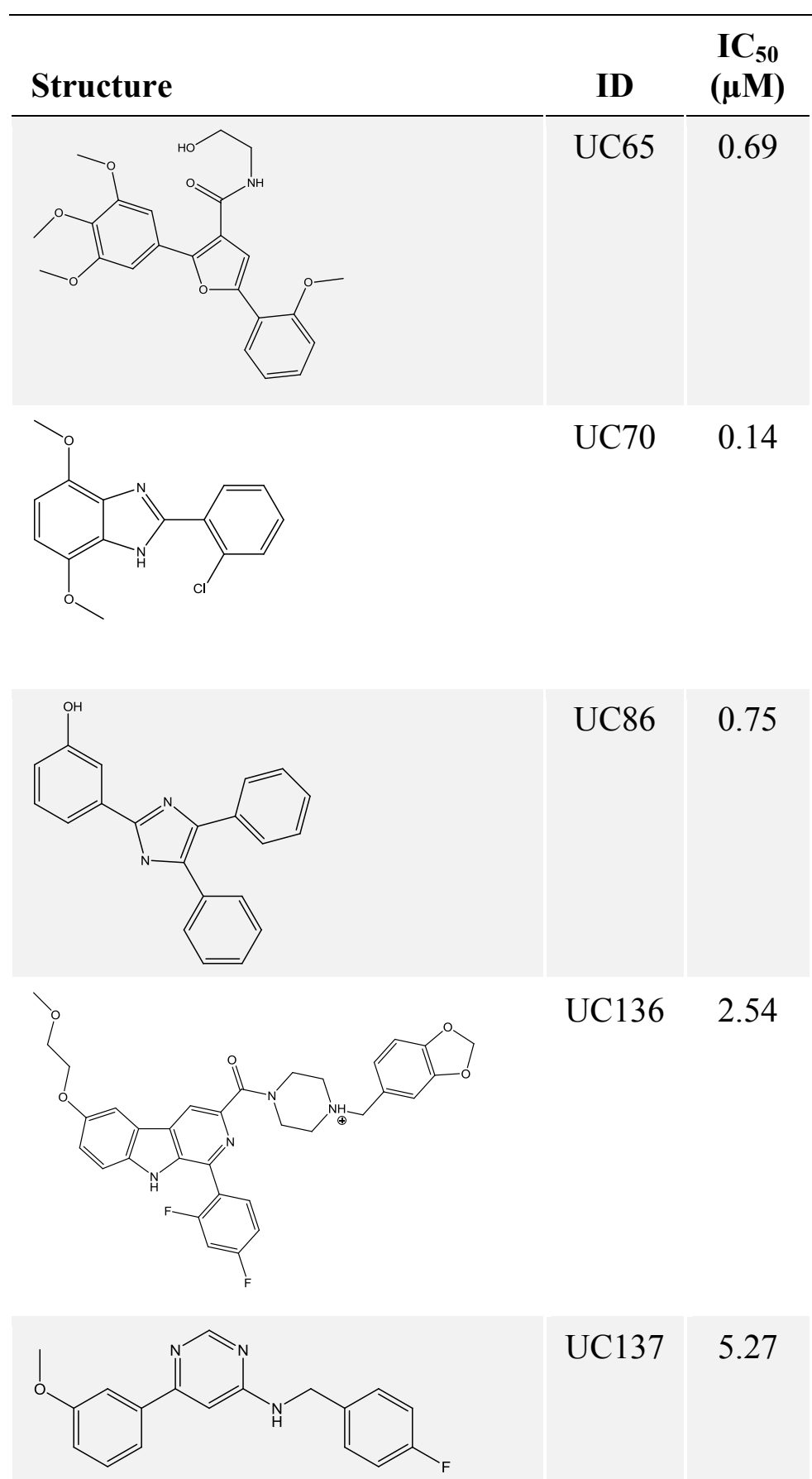

\begin{tabular}{l|l} 
UC137 & 5.27
\end{tabular} 
Table 2-9. Continued

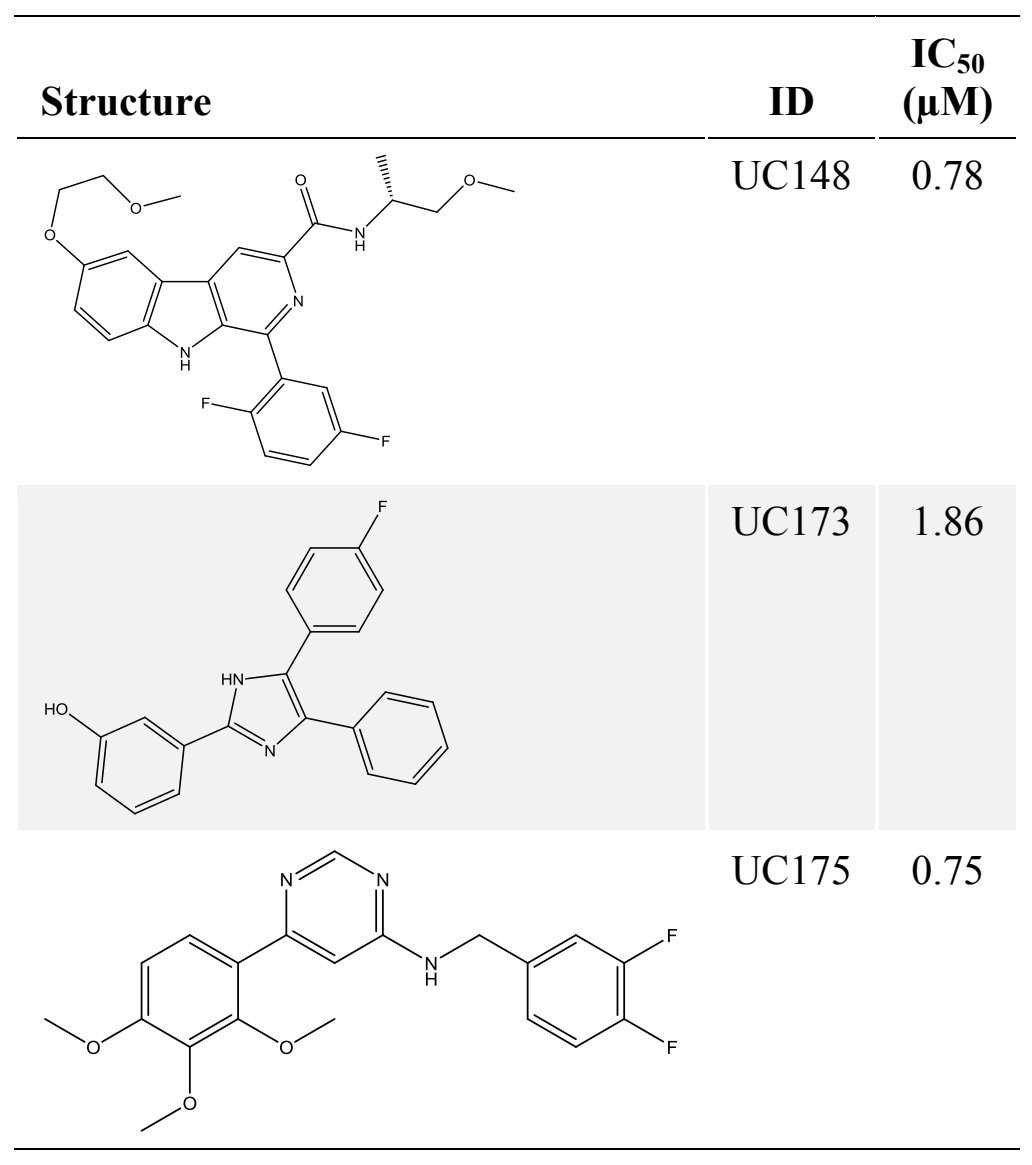

Note: *Compound demonstrated mixed activity. 
The contours for all of the presented CoMFA and CoMSIA models can be found in the Appendix (Figures A-1 through A-17).

While the hCNT3 model proved wholly unsuccessful at discovering any hCNT3 hits, it appeared to describe a general pharmacophore for equilibrative nucleoside transporter activity, resulting in thirteen hENT1 and three hENT4 hits (Chapter 3).

In comparing the hCNT3 pharmacophore (Figure 2-17) with the flavonoid hENT1 pharmacophore (Figure 2-3), one notes a great deal of similarity. This likely pointed to the nature of flavonoid compounds and their broad spectrum of activity against many biological targets. Even so, the hCNT1 pharmacophore performed well, despite poor statistics, with a hit rate of $2.08 \%$. The pyridone chemical class can serve as a starting point for further structure-activity elucidation relative to hCNT1. 


\section{CHAPTER 3. BIOLOGICAL STUDIES}

\section{Introduction}

A number of factors should be considered when selecting an assay system for screening chemical compounds for biological activity. First, one must understand that affinity does not always correlate to efficacy. For one, while cell biology has well characterized many pathways within cells, there remain mechanisms which are not understood or remain uncharacterized. In vitro chemical or enzyme assays are low in cost, but do not take into consideration the "cellular veil" i.e. the complex interactions and interdependence of inter- and intracellular signaling. Whole cell assays provide a means to test direct interaction with the cell, but do not address metabolism or biological distribution. Animal models can elucidate efficacy and proof of concept, but are expensive and many variables such as species variation, metabolism, bioavailability, and pharmacokinetics must be explored before utilizing animal models. ${ }^{77}$

As for the nucleoside transporters, a number of methods have been used in the literature over the years. Since most cell lines identified to date possess more than one type of transporter, they are not ideal for screening. Ward et al. established a transporter deficient pig kidney cell line (PK15NTD) and stably transfected it with cloned hENT1 and hENT2. ${ }^{29}$ They, along with Wang et al. proceeded to express the remaining transporters, with the exception of hENT3, in PK15NTD cells. ${ }^{70}$ This allowed for each transporter to be screened individually. In some cases rat myoblast cells $(\mathrm{H} 9 \mathrm{c} 2)^{78}$ cells were used for testing of ENT2 function, but results were later cross-validated with PK15hENT2 cells.

\section{Experimental Methods}

Cell lines were grown and maintained in Eagle's Minimum Essential Media (EMEM) with 10\% fetal bovine serum and Geneticin G418 selector antibiotic at $5 \mathrm{mg} / \mathrm{ml}$. A kill curve was run on the parental PK15NTD cell line and it necessitated up to 15 $\mathrm{mg} / \mathrm{ml}$ to achieve $100 \%$ cell mortality. Lower concentrations can be utilized with purer, more potent grades of G418. The G418 from KES Scientific, the supplier used for these studies, averaged only $70 \%$ potent.

Also, to maintain expression of the transfected cells, they were reselected every 60 days or a new vial of stock cells used for further experiments. Cells were plated at 40,000 cells per well in Corning's clear polystyrene forty-eight well plates and incubated at $37^{\circ} \mathrm{C}$ and $5 \% \mathrm{CO}_{2}$ overnight. For screening, $10 \mu \mathrm{M}$ dilutions of compounds were prepared in either a sodium containing or sodium free buffer (Table 3-1) from 1mM stocks in dimethyl sulfoxide (DMSO). For dose-response experiments, serial dilutions were made and tested.

Screening and dose-response assays, except where indicated, were carried out technical triplicate. Compounds were tested using a radio-ligand uptake assay, utilizing 
Table 3-1. Uptake assay buffers

\begin{tabular}{lccc}
\hline $\begin{array}{l}\text { Reagent for } \\
\text { Sodium } \\
\text { Buffer }\end{array}$ & $\begin{array}{c}\text { Final Concentration } \\
(\mathbf{m M})\end{array}$ & $\begin{array}{c}\text { Reagent for } \\
\text { Sodium Free } \\
\text { Buffer }\end{array}$ & $\begin{array}{c}\text { Final Concentration } \\
(\mathbf{m M})\end{array}$ \\
\hline $\mathrm{NaCl}$ & 120 & $\mathrm{Choline} \mathrm{Chloride}$ & 120 \\
$\mathrm{Tris}-\mathrm{HCl}$ & 20 & $\mathrm{Tris}-\mathrm{HCl}$ & 20 \\
$\mathrm{~K}_{2} \mathrm{PO} 4$ & 3 & $\mathrm{~K}_{2} \mathrm{PO}_{4}$ & 3 \\
$\mathrm{Glucose}$ & 10 & $\mathrm{Glucose}$ & 10 \\
$\mathrm{MgCl}_{2}$ & 1 & $\mathrm{MgCl}_{2}$ & 1 \\
$\mathrm{CaCl}_{2}$ & 1 & $\mathrm{CaCl}_{2}$ & 1 \\
\hline
\end{tabular}

Note: $\mathrm{pH}$ adjusted with hydrochloric acid $(\mathrm{HCl})$ or sodium hydroxide $(\mathrm{NaOH})$ to 7.4 or 6.0 for hENT4 
previously established transgenic PK15NTD porcine cells expressing individual recombinant human (h)ENT ${ }^{29}$ or $(\mathrm{h}) \mathrm{CNT}^{79}$ and/or H9c2 rat cells expressing native rENT2. For the rat cells, rENT1 was inhibited with $100 \mathrm{nM}$ NBMPR prior to addition of analogues to avoid participation of the low level of ENT1 expressed by these cells. Decreased uptake of the ${ }^{3} \mathrm{H}[5-]$ uridine indicated a "hit" and was confirmed by repeating the assay with serially diluted concentrations of the compounds to establish doseresponse curves. Positive controls were run with every plate. Table 3-2 lists the inhibitors and concentration used for each transporter. Figure 3-1 illustrates uptake assay procedure.

Readings were interpreted as percentage uptake relative to untreated cells. All chemicals used were of reagent grade and purchased from Sigma-Aldrich or Fisher Scientific.

\section{Results}

\section{hCNT3 Pharmacophore Selections Screening}

$10 \mathrm{mM}$ stock solutions in DMSO were purchased from the University of Cincinnati. Working stock solutions of $1 \mathrm{mM}$ were prepared for all compounds in DMSO and all subsequent dilutions were in uptake assay buffer. All wells were run in triplicate. rENT2 was used and hits tested against hENT2.

Figures 3-2 through 3-13 show the biological screening data. Some of the hits like UC9 for example, turned out to be false positives. Likely, the false positives which showed time dependent inhibition of uridine uptake were possible substrates and competed with the tritiated uridine for uptake. UC9, for example, appeared to markedly inhibit all tested ENTs. When retested using a twenty minute incubation period to confirm the hit, the apparent inhibition disappeared.

Due to lack of hENT2 cells at the time screening began H9c2 cells expressing native rENT2 were used. Hits were cross screened against hENT2, but none of the hits maintained significant potency (Figure 3-14). Dose-response assays were then conducted on confirmed hits. Figure 3-15 and Figure 3-16 show the dose-response curves for hENT1 and hENT4 hits, respectively.

Several compounds exhibited complex kinetics, suggesting perhaps that they are allosteric modulators and/or are mixed acting. Further kinetic studies would be required to define the mechanism of inhibition.

The only hits of significant potency were those which hit hENT1, some of which were of sub-micromolar potency, and hENT4. While the hENT1 hits were of diverse chemical structures (Table 2-9), the hENT4 hits were all of the same chemical class, substituted imidazo[4,5f]quinolones (Figure 3-17). 
Table 3-2. Inhibitor controls used for uptake assays

\begin{tabular}{lcc}
\hline Transporter & Inhibitor & Concentration \\
\hline hENT1 & NMBPR & $100 \mathrm{nM}$ \\
hENT2/rENT2 & Dipyridamole & $1 \mu \mathrm{M}$ \\
hENT4 & WL30 & $1 \mu \mathrm{M}$ \\
hCNT1 & Phlorizin & $250 \mu \mathrm{M}$ \\
hCNT2 & Phlorizin & $250 \mu \mathrm{M}$ \\
hCNT3 & Phlorizin & $250 \mu \mathrm{M}$ \\
\hline
\end{tabular}




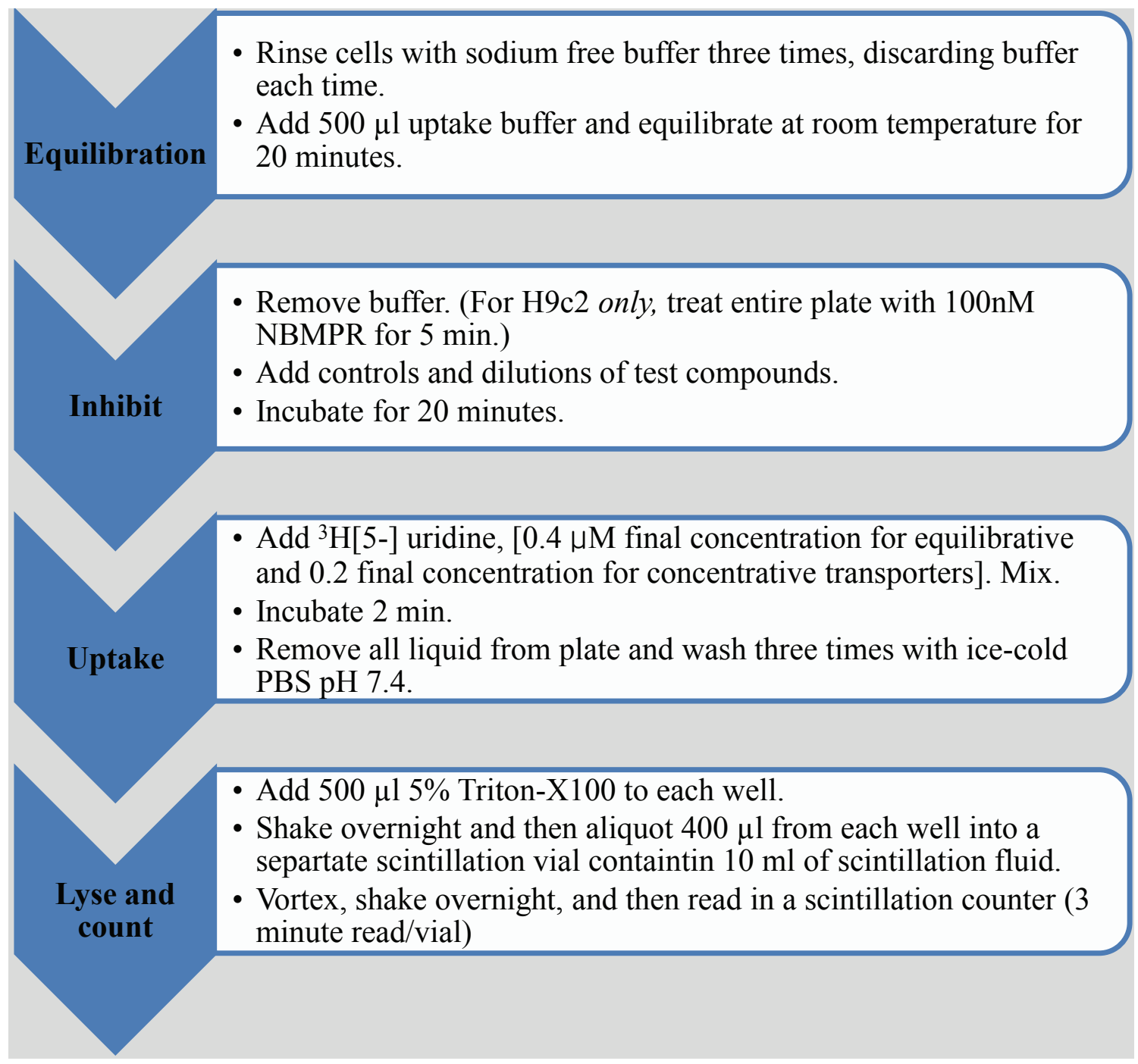

Figure 3-1. Uptake assay procedure workflow

Note: Total volume per/well was $500 \mu \mathrm{l}$. Volumes of test compounds, NBMPR, other standards, and tritiated uridine were adjusted accordingly. 


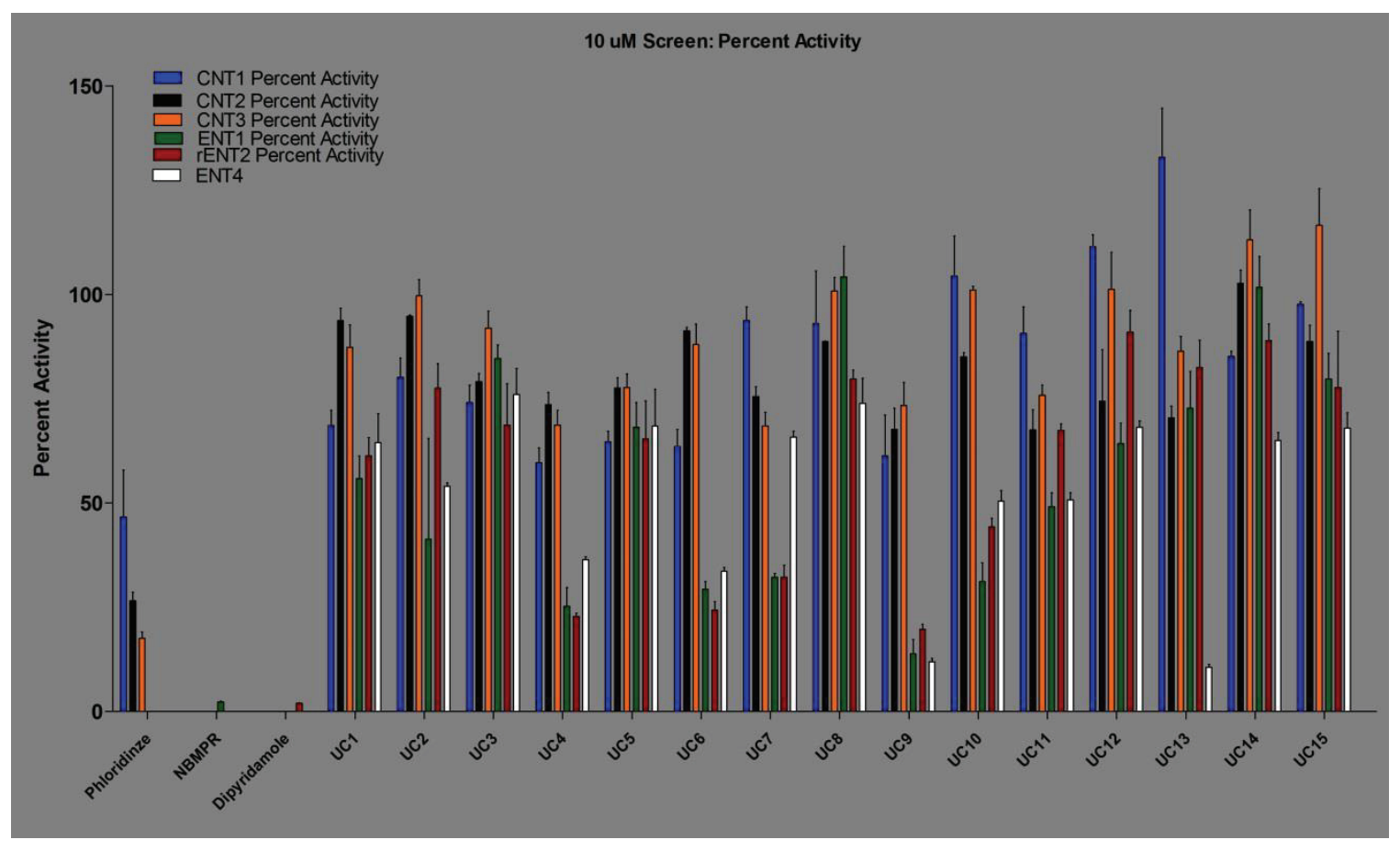

Figure 3-2. UC1-UC15 screening results 


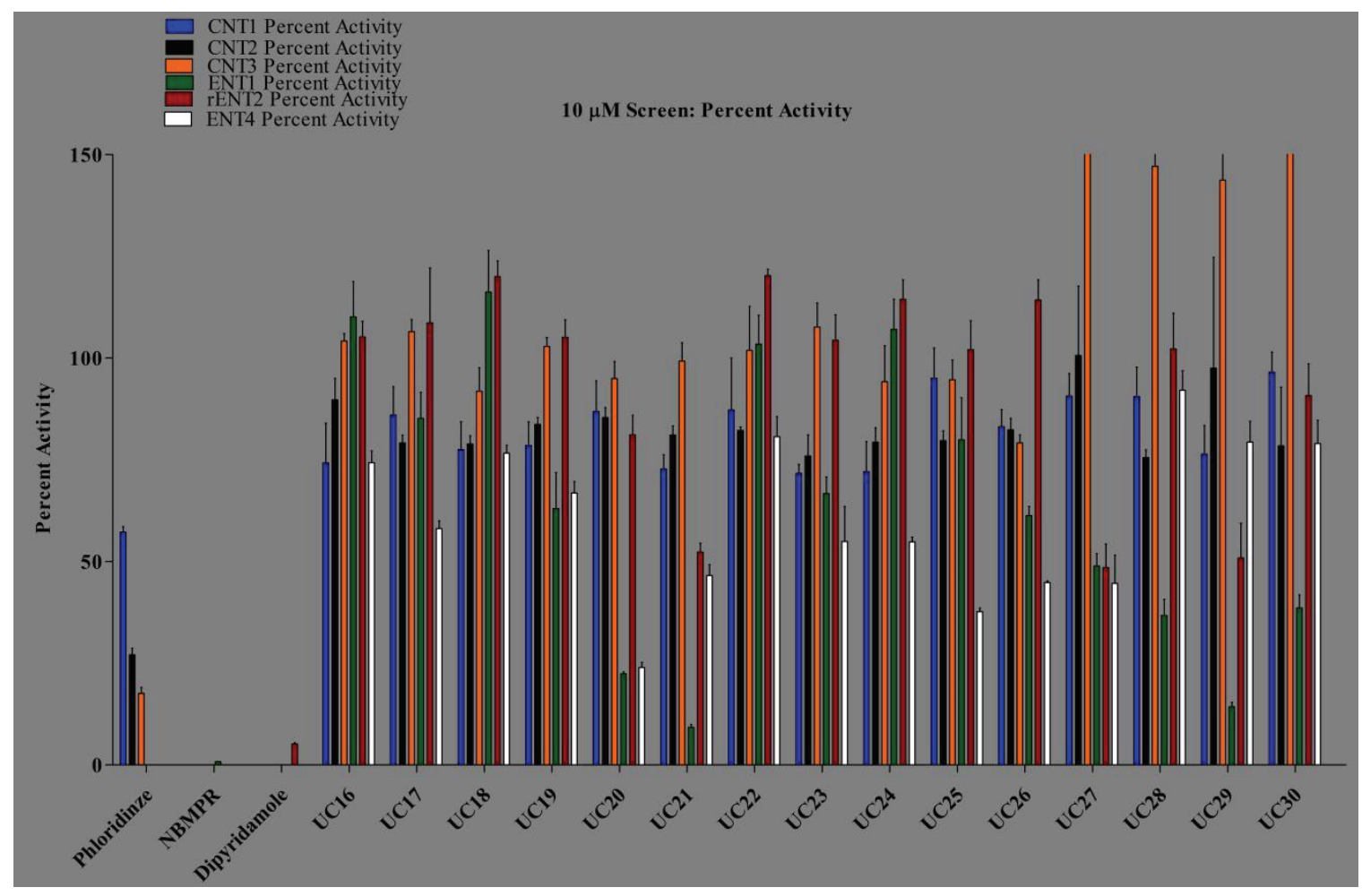

Figure 3-3. UC16-UC30 screening results 


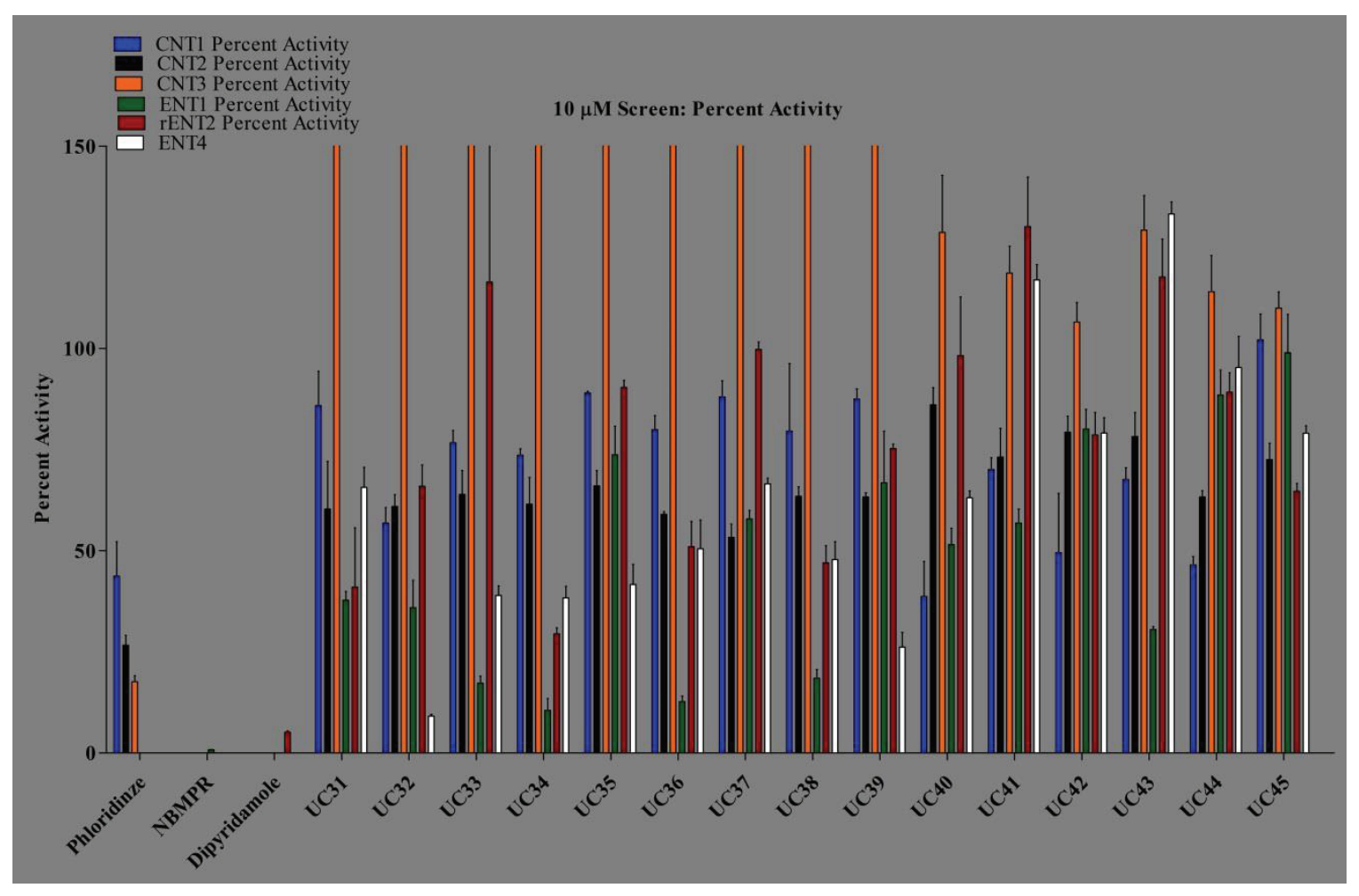

Figure 3-4. UC31-UC45 screening results 


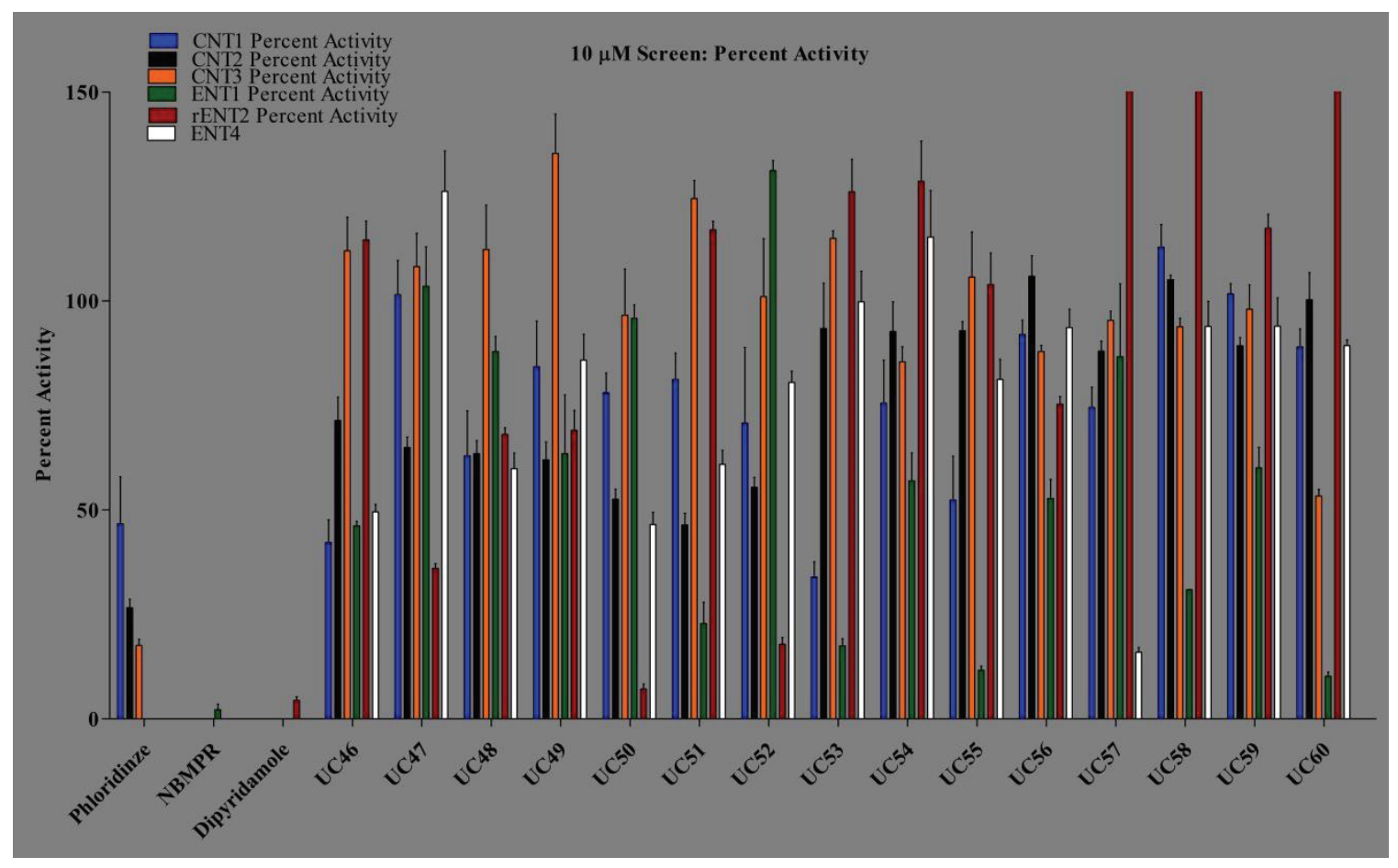

Figure 3-5. UC46-UC60 screening results 


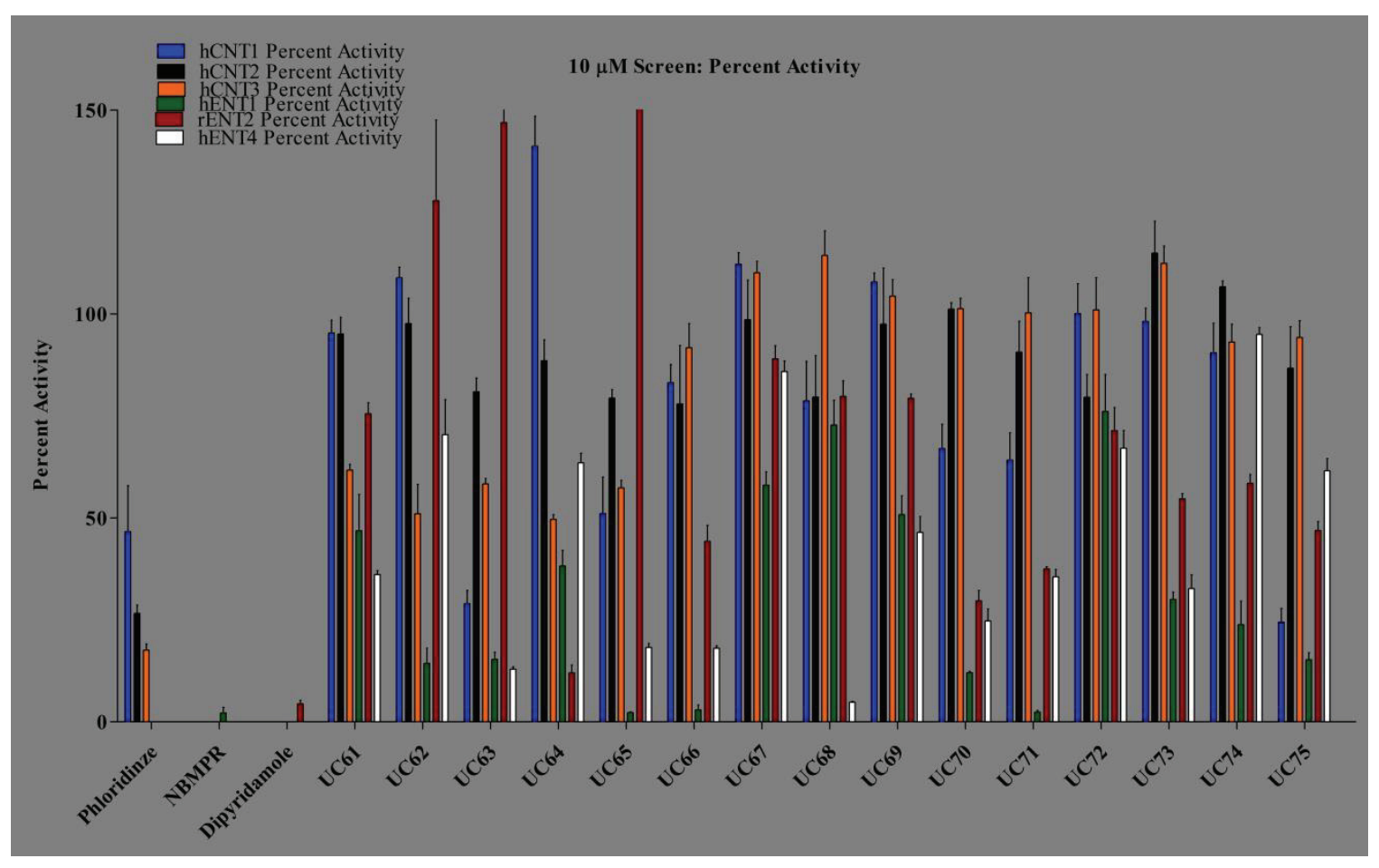

Figure 3-6. UC61-UC75 screening results 


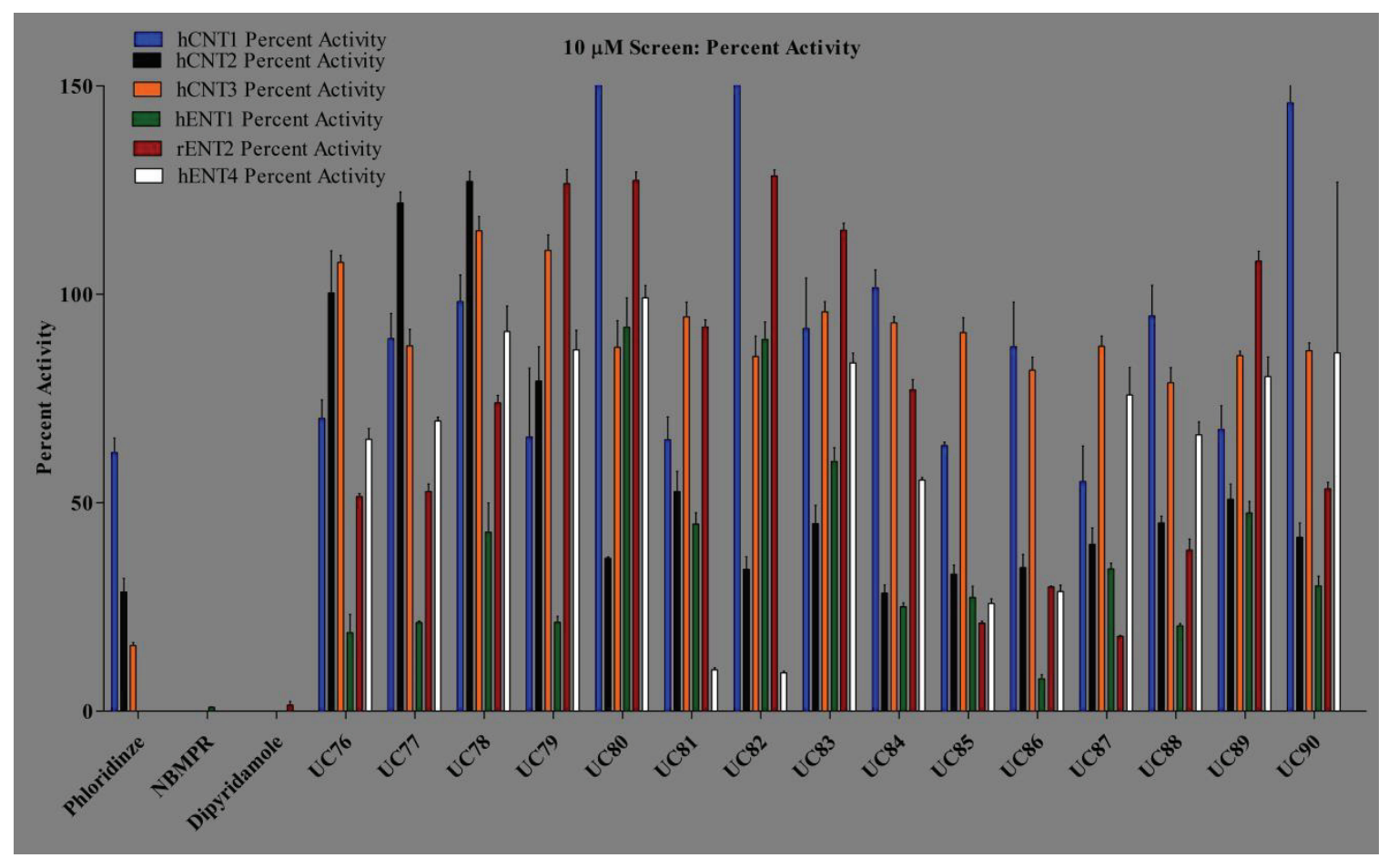

Figure 3-7. UC76-UC90 screening results 


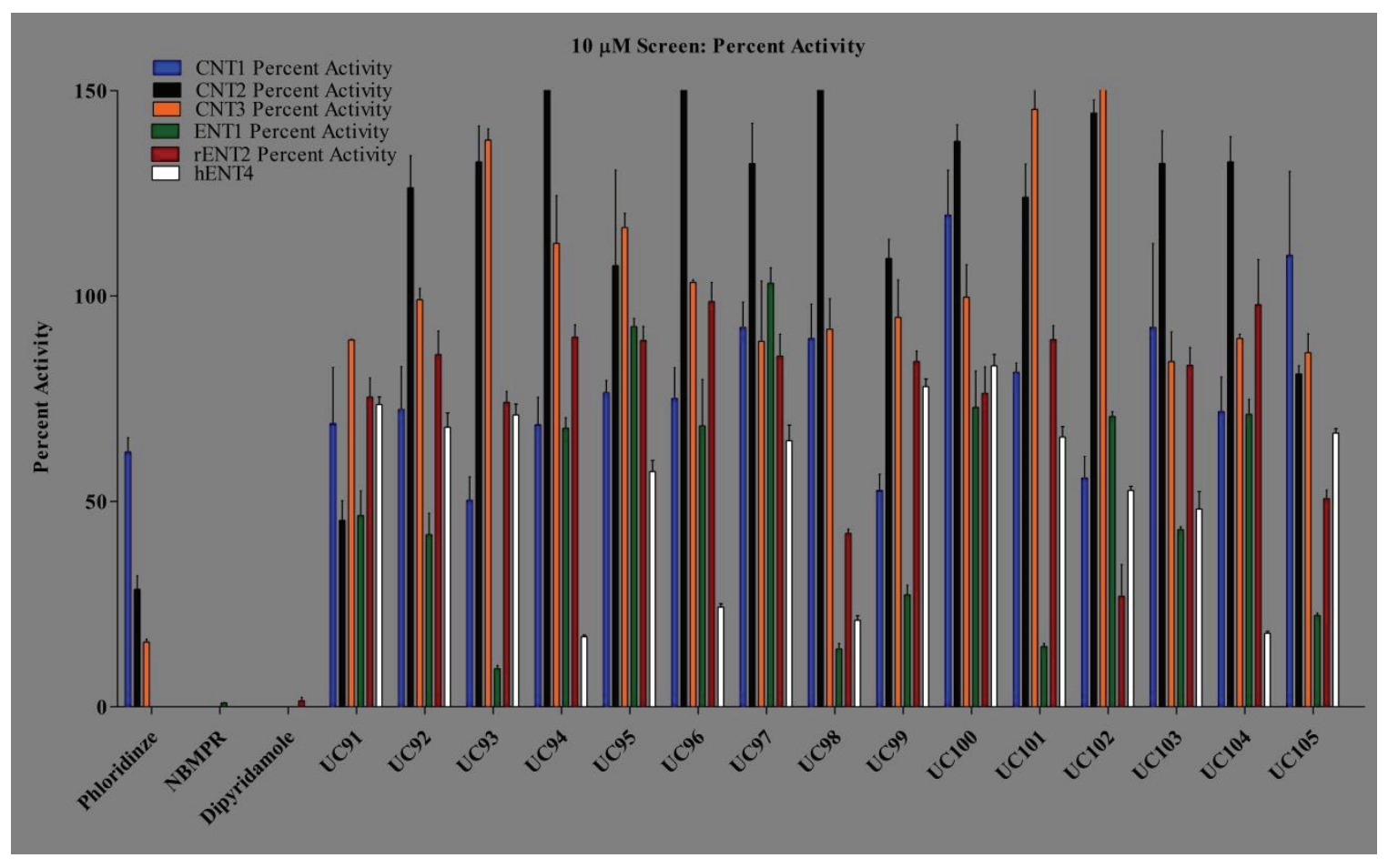

Figure 3-8. UC91-UC105 screening results 


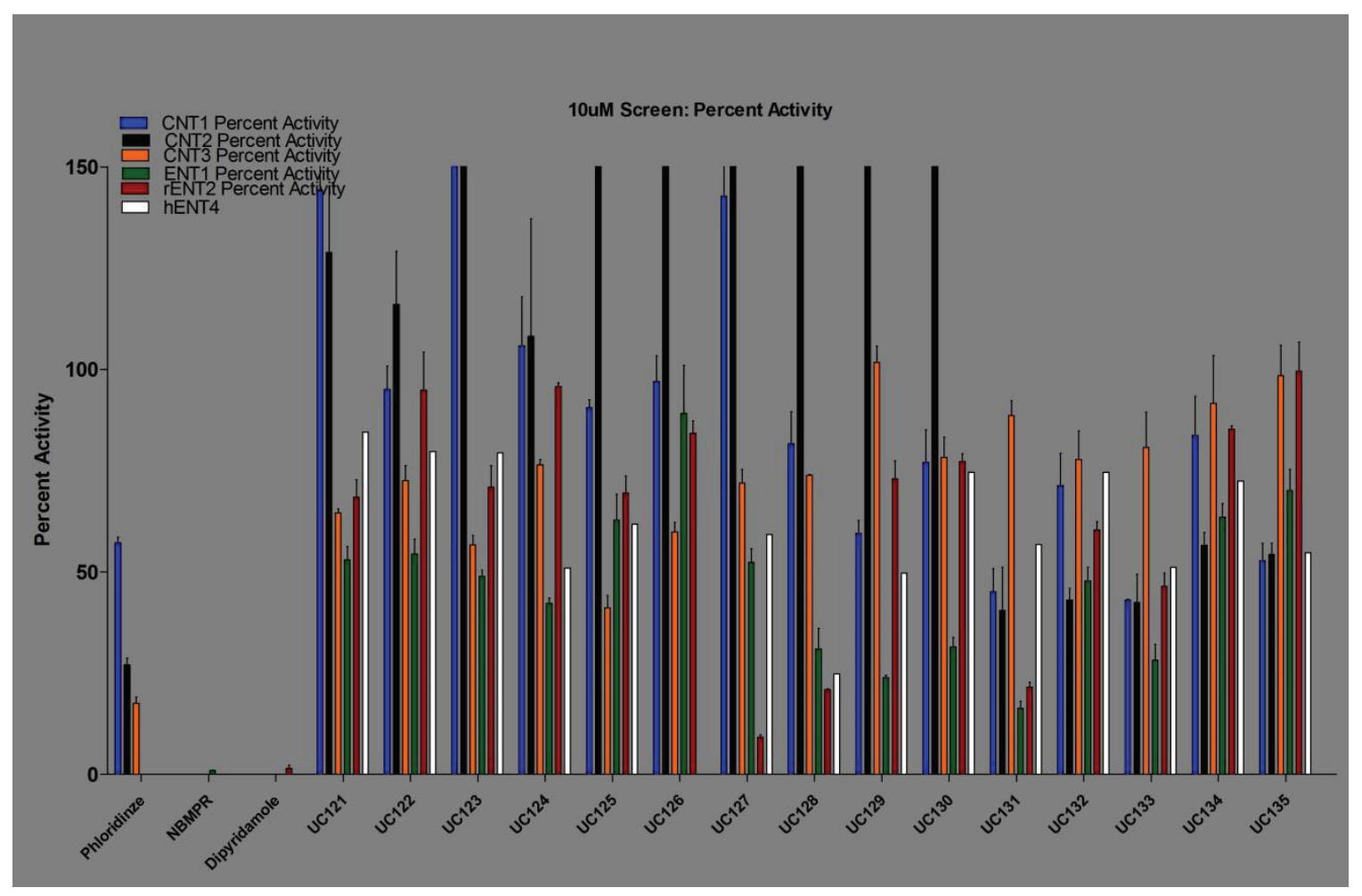

Figure 3-9. UC121-UC135 screening results 


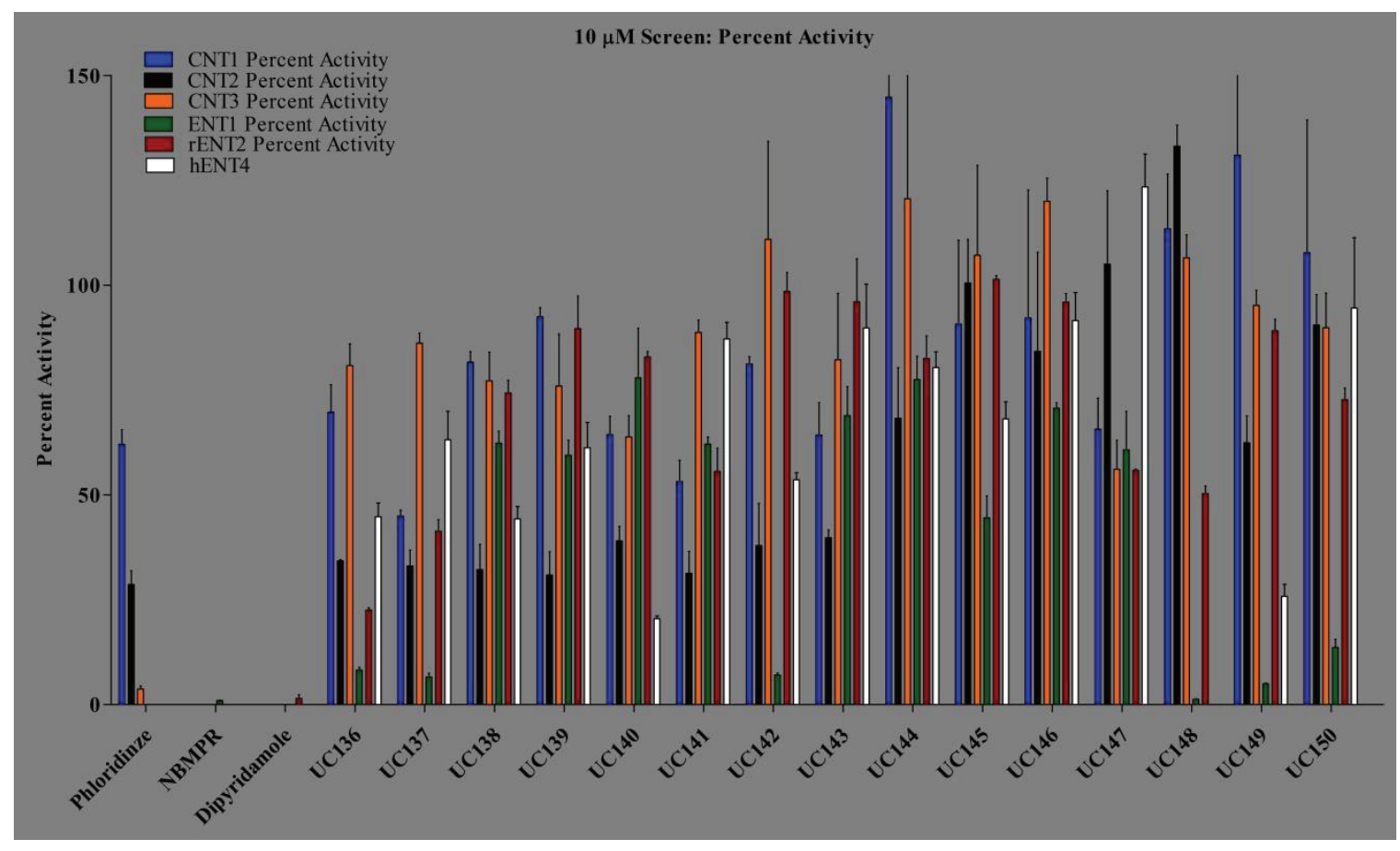

Figure 3-10. UC136-UC150 screening results 


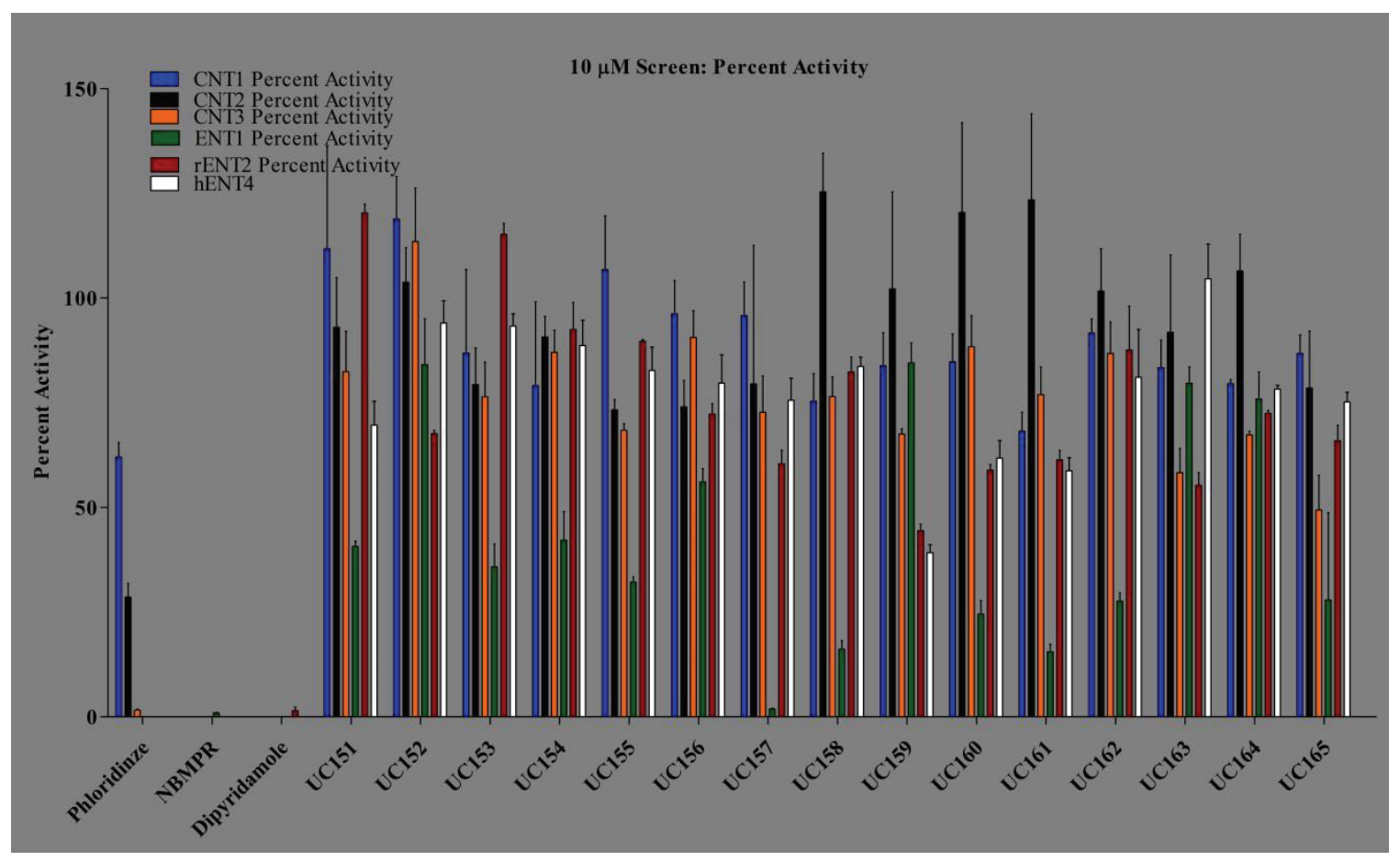

Figure 3-11. UC152-UC165 screening results 


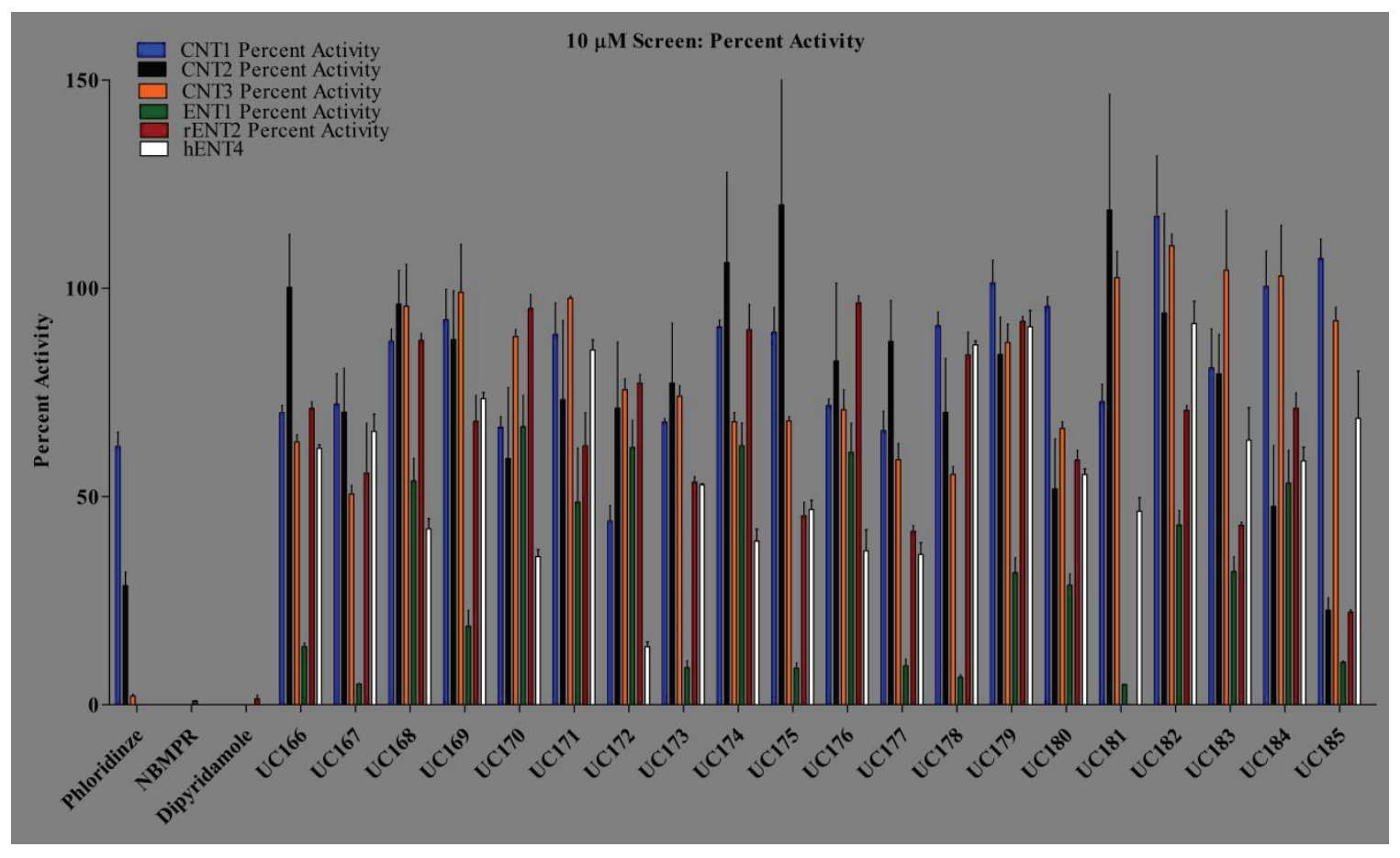

Figure 3-12. UC166-UC185 screening results 


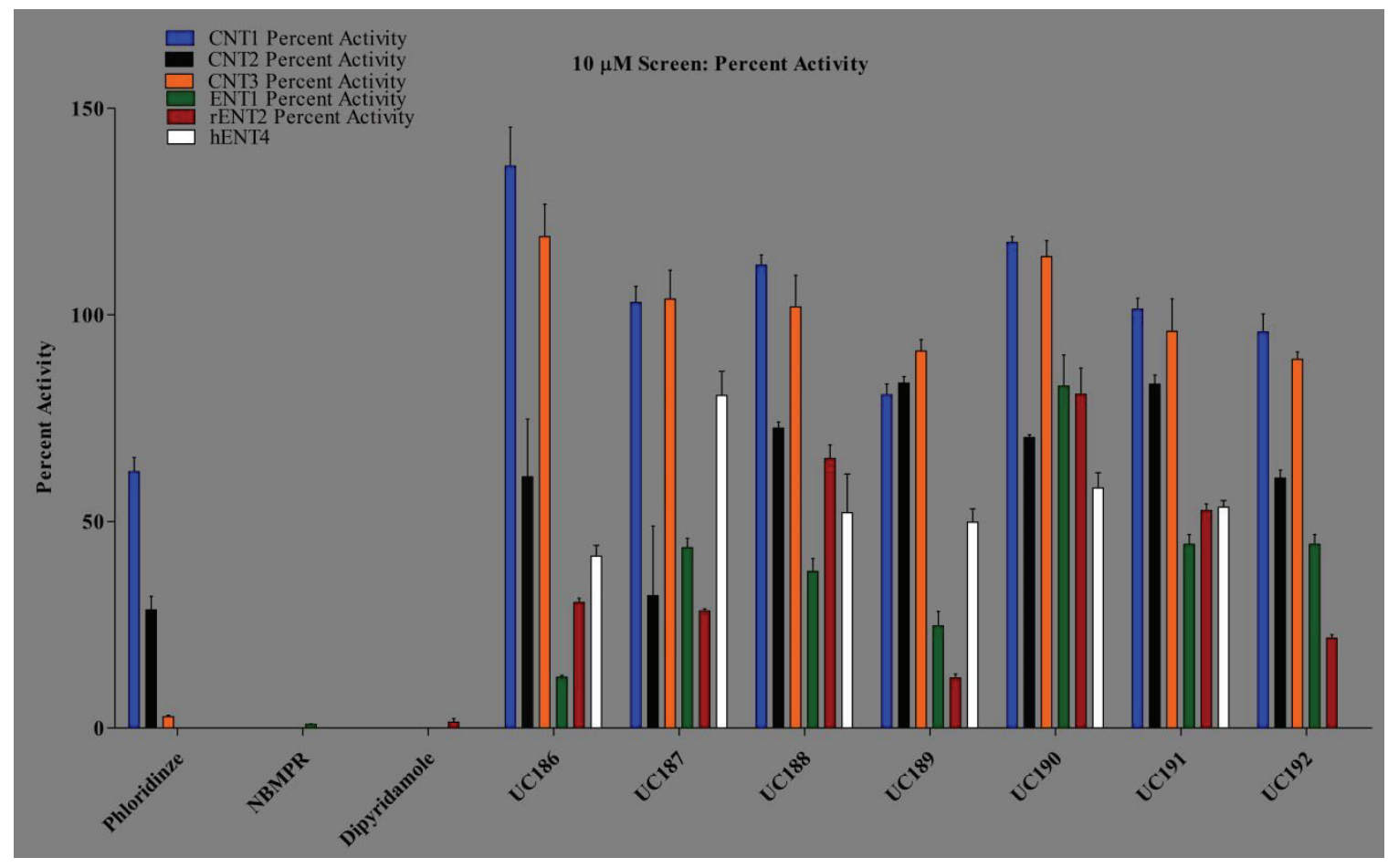

Figure 3-13. UC186-UC192 screening results 


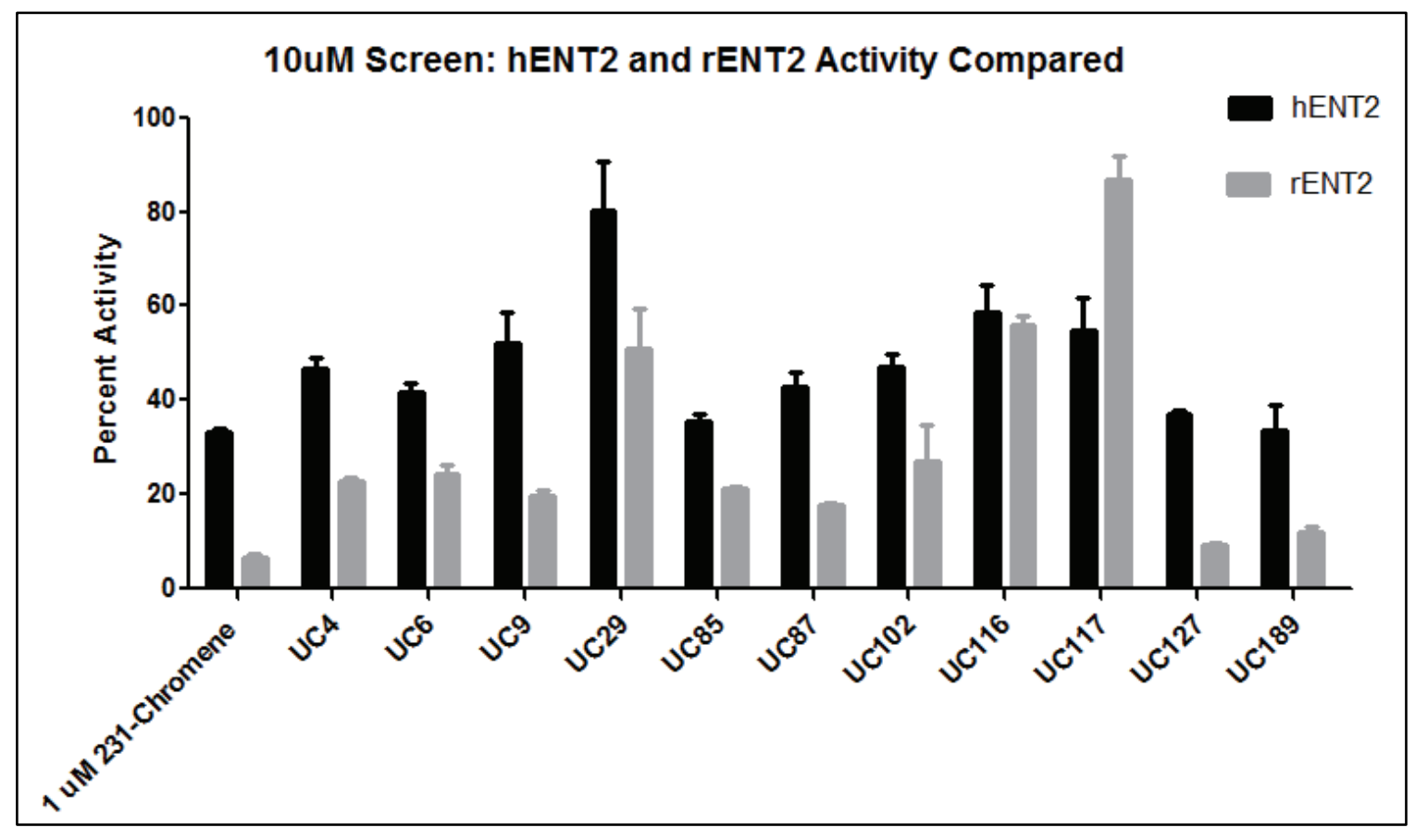

Figure 3-14. Cross screen of rENT2 hits against hENT2 
Figure 3-15. hENT1 dose response curves

Note: $\mathrm{IC}_{50} \mathrm{~s}^{*}$ : (A) $54.4 \mathrm{nM}$ (B) $173.2 \mathrm{nM}$ (C) $309.2 \mathrm{nM}$ (D) $156.4 \mathrm{nM}$ (E) $1455 \mathrm{nM}$ (F) 65.5\% Maximal Inhibition at $20 \mu \mathrm{M}(\mathrm{G}) 3001 \mathrm{nM}(\mathrm{H})$ 84.6\% Maximal Inhibition at 20 $\mu \mathrm{M}$ (I) $692.4 \mathrm{nM}(\mathrm{J}) 142.7 \mathrm{nM}(\mathrm{K})$ 95.1\% Maximal Inhibition at $10 \mu \mathrm{M}$ (L) $747.8 \mathrm{nM}$ (M) $2542 \mathrm{nM}(\mathrm{N})$ 92.6\% Maximal Inhibition at $15 \mu \mathrm{M}(\mathrm{O}) 769.0 \mathrm{nM}(\mathrm{P}) 1681 \mathrm{nM}(\mathrm{Q})$ $745.1 \mathrm{nM}$

*95\% CI [M] (A) $1.455 \mathrm{e}-008$ to $2.033 \mathrm{e}-007$ (B) $2.235 \mathrm{e}-008$ to $1.342 \mathrm{e}-006$ (C) $1.979 \mathrm{e}-$ 007 to $4.829 \mathrm{e}-007$ (D) $2.773 \mathrm{e}-008$ to $8.818 \mathrm{e}-007$ (E) $7.664 \mathrm{e}-007$ to $2.764 \mathrm{e}-006$ (F) $2.332 \mathrm{e}-007$ to $0.003096(\mathrm{G}) 1.121 \mathrm{e}-006$ to $8.036 \mathrm{e}-006(\mathrm{H}) 2.208 \mathrm{e}-006$ to $5.160 \mathrm{e}-006$ (I) $5.001 \mathrm{e}-007$ to $9.586 \mathrm{e}-007$ (J) $8.865 \mathrm{e}-008$ to $2.298 \mathrm{e}-007$ (K) $4.940 \mathrm{e}-007$ to $1.793 \mathrm{e}-006$ (L) $4.591 \mathrm{e}-007$ to $1.218 \mathrm{e}-006(\mathrm{M}) 1.290 \mathrm{e}-006$ to $5.012 \mathrm{e}-006(\mathrm{~N}) 2.209 \mathrm{e}-006$ to $1.259 \mathrm{e}-005$ (O) $5.797 \mathrm{e}-007$ to $1.020 \mathrm{e}-006(\mathrm{P}) 1.259 \mathrm{e}-006$ to $2.245 \mathrm{e}-006(\mathrm{Q}) 5.267 \mathrm{e}-007$ to $1.054 \mathrm{e}-$ 006 


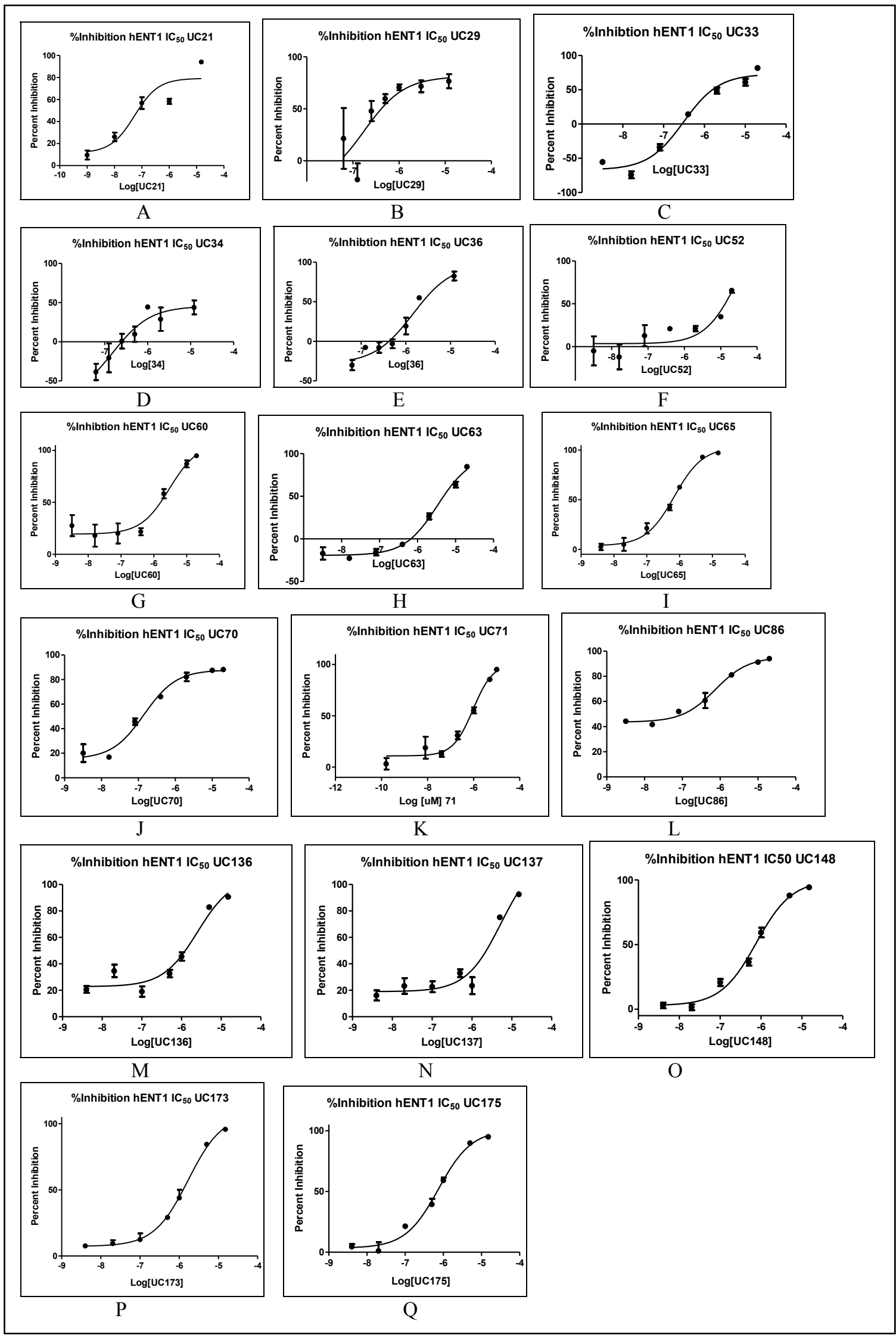




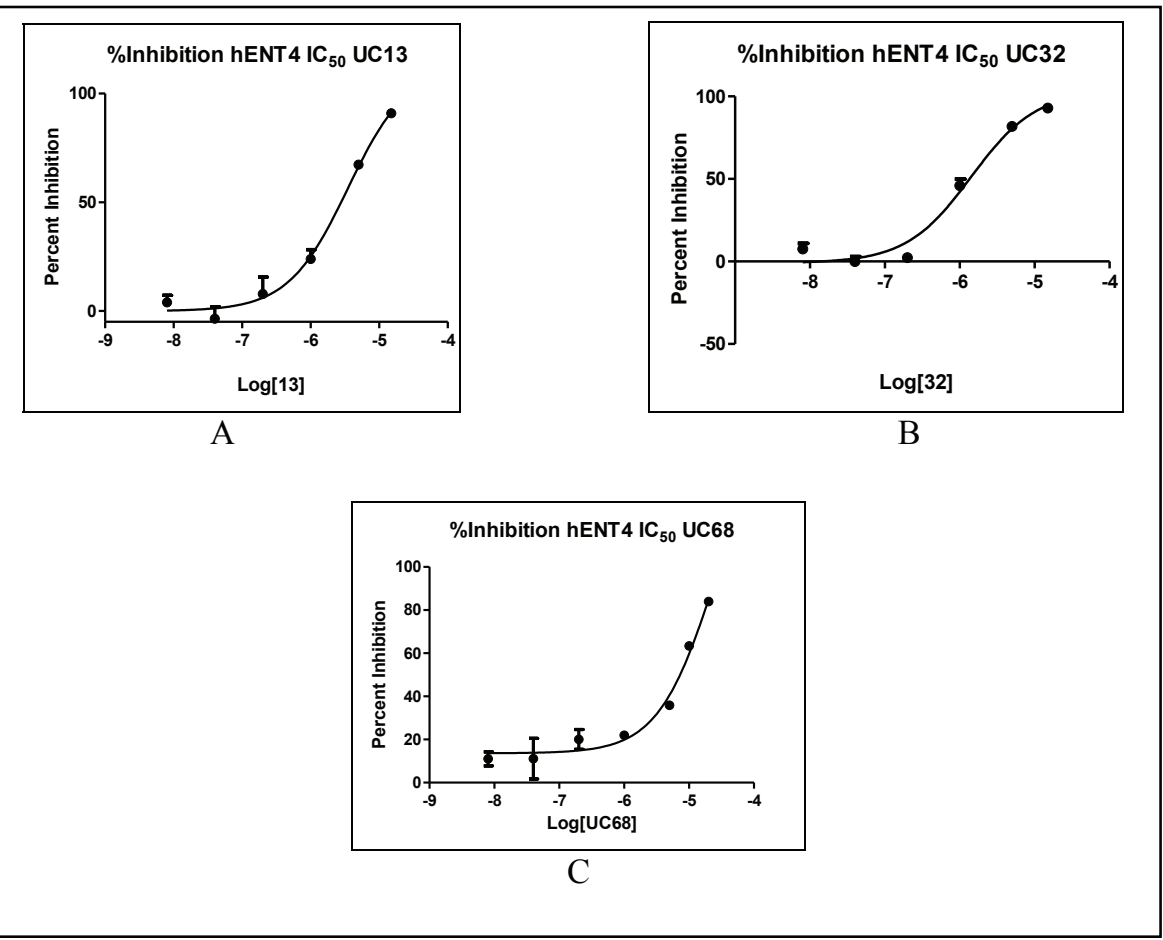

\section{Figure 3-16. hENT4 dose response curves}

Note: $\mathrm{IC}_{50} \mathrm{~S} *$ : (A) $3.76 \mu \mathrm{M}$ (B) $1.43 \mu \mathrm{M}$ (C) $24.2 \mu \mathrm{M}$

*95\% CI [M] (A) 1.893e-006 to $6.363 \mathrm{e}-006$ (B) 8.776e-007 to $2.318 \mathrm{e}-006$ (C) $7.856 \mathrm{e}-$ 006 to $7.462 \mathrm{e}-005$

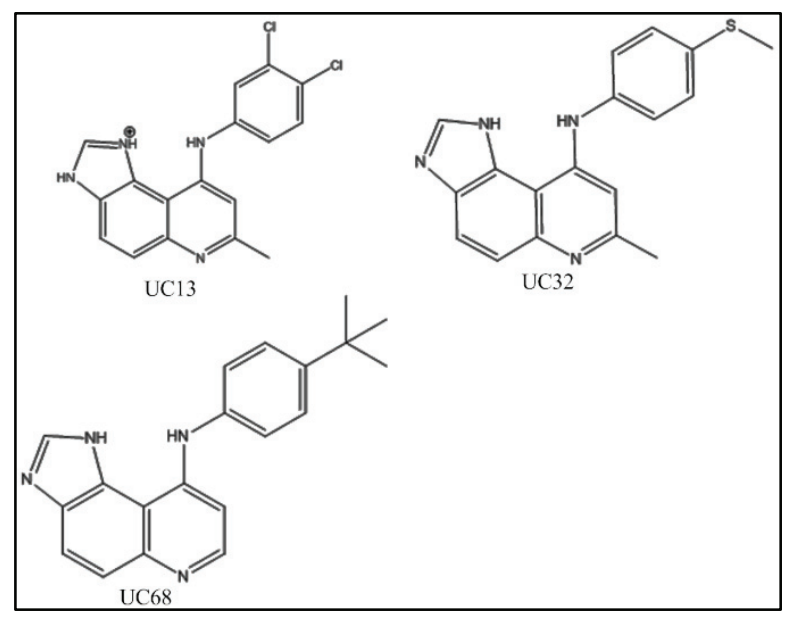

Figure 3-17. hENT4 hit structures 


\section{hCNT1 Pharmacophore Selections Screening}

From the virtual screening of the hCNT1 pharmacophore (referred to as B set), 96 compounds were tested for biological activity. All compounds were tested in triplicate and hits confirmed with dose response assays. A "hit" was defined as at least $75 \%$ inhibition at $10 \mu \mathrm{M}$. The screening results are depicted in Figures 3-18 through 3-22.

Using the $75 \%$ inhibition cut-off, only two molecules met the requirement for activity. Both $58 \mathrm{~B}$ and $82 \mathrm{~B}$ showed selectivity for hCNT1. Their $\mathrm{IC}_{50}$ s were calculated using a dose response assay Figure 3-23.

Both 58B and 82B belong to the same pyridone chemical class (Figure 3-24), supporting the supposition that derivatives can be used to explore the structure-activity relationship and build a larger database of molecules with hCNT1 activity, thus improving the ability of the pharmacophore model to predict activity.

\section{Vibrio Cholerae Selections Screening}

To compare the efficacy of the homology model to the published crystal structure, 96 compounds drug-like were selected for biological testing against the CNT transporters. Figures 3-25 through 3-28 show the biological screening data.

No compounds were selected for dose dependent assays, as none showed greater than $75 \%$ inhibition. V92 appeared to be the strongest hit, and only inhibited CNT1 by $73 \%$. As this was not a human transporter, the lack of hits served as confirmation of species selectivity and that any hits found with the homology model were not likely due to the similarity in veCNTs and hCNTs.

\section{hCNT1 Homology Model Selections Screening}

One hundred seventy-seven molecules were selected for biological testing and each was tested in triplicate wells and against all of the concentrative transporters using the same procedures previously described. Figures 3-29 through 3-34 show the biological screening data.

\section{Summary and Conclusion}

The CNT3 pharmacophore failed to identify hCNT3 potent compounds. Reexamination of some of the compounds used to build the model indicated that they were likely competitive substrates rather than inhibitors. This supposition was supported by measuring uptake inhibition of some of the hits at more than one time point. UC9, for example, showed significant inhibition of all equilibrative transporters during initial screening (Figure 3-2) Noting that the first few screens were done with the $15 \mathrm{~min}$. preincubation of the test compounds and then hits were repeated at $20 \mathrm{~min}$ time points, all 


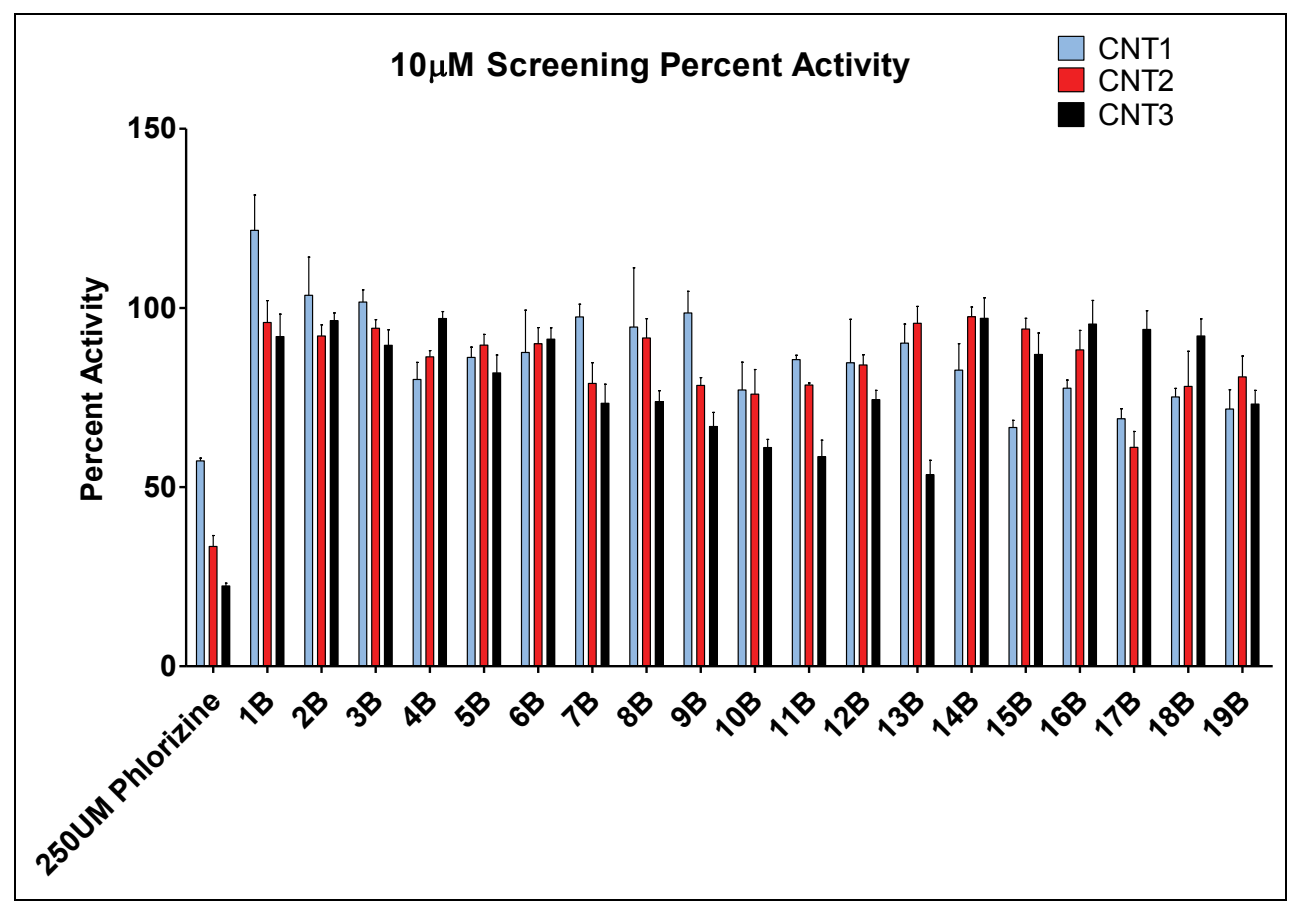

Figure 3-18. B1-19 screening results

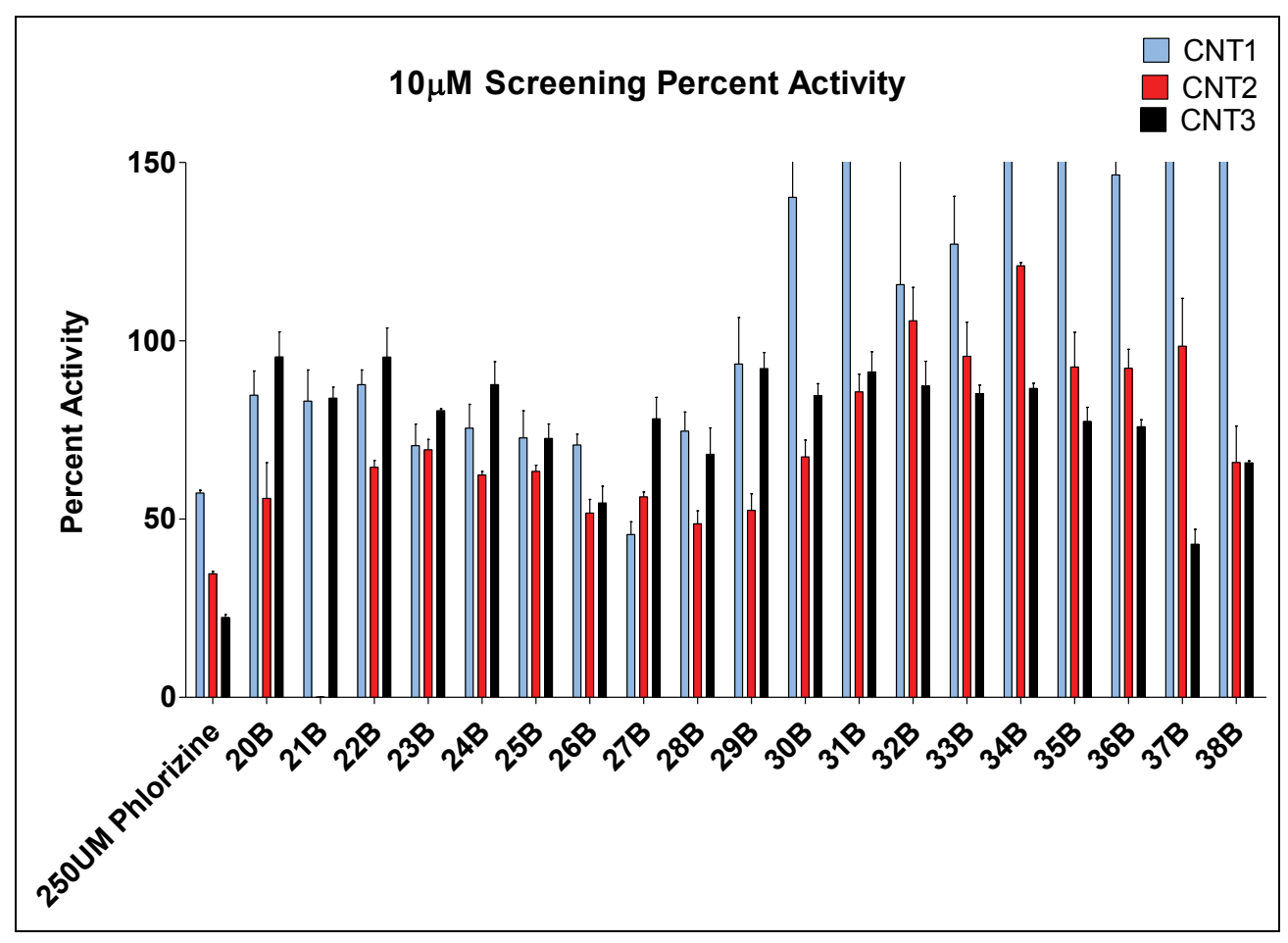

Figure 3-19. 20B-38B screening results 


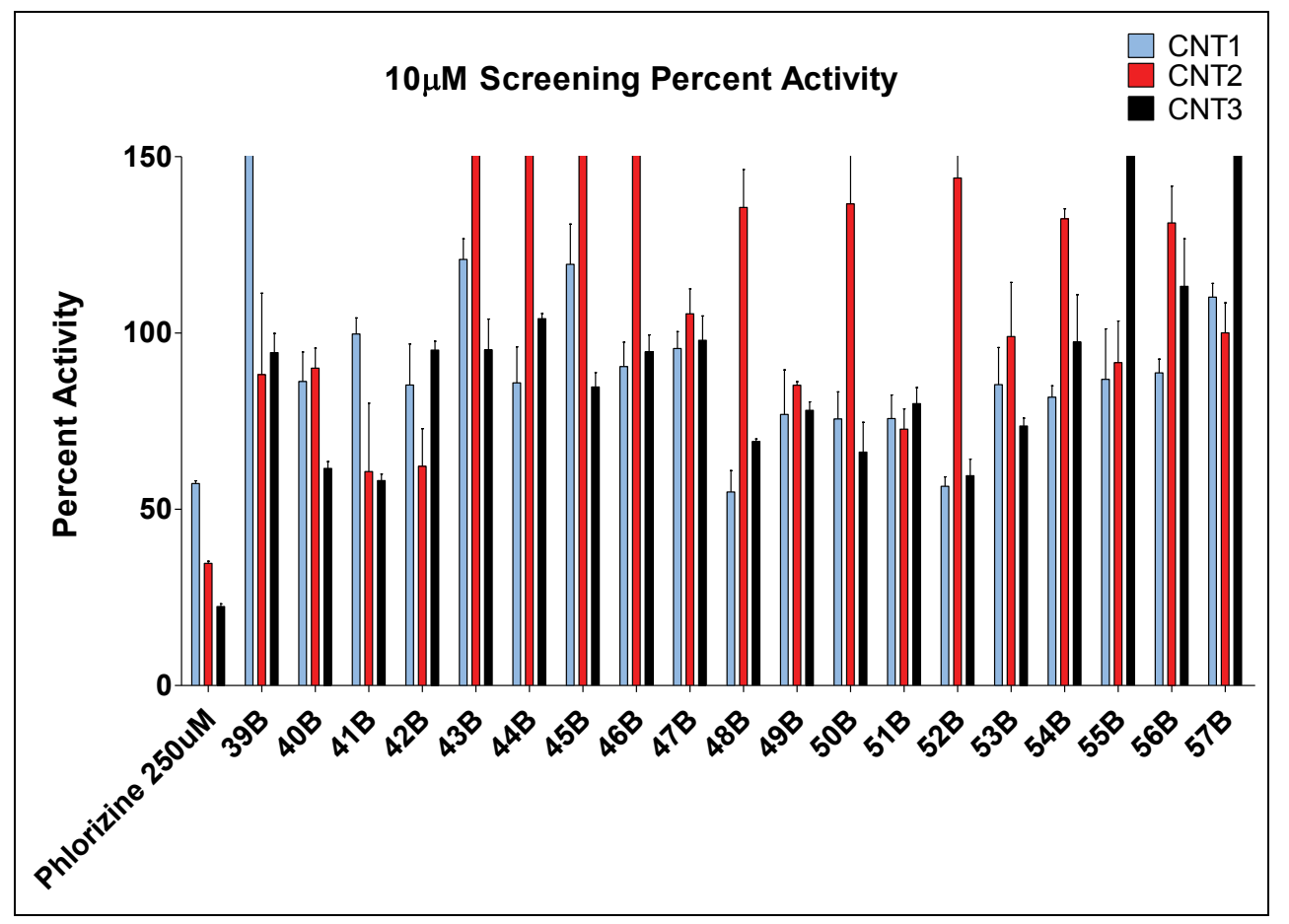

Figure 3-20. 39B-57B screening results

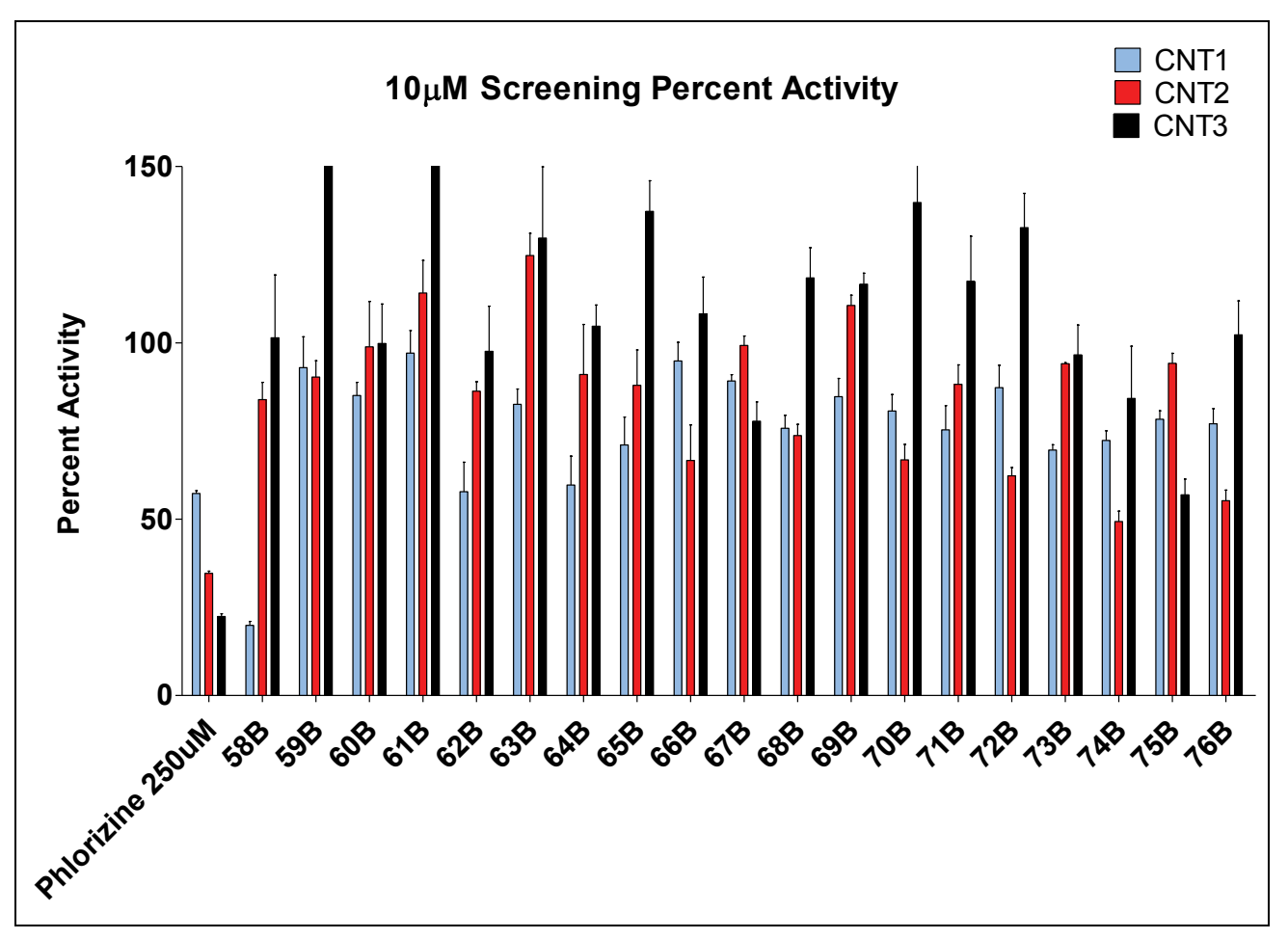

Figure 3-21. 58B-76B screening results 


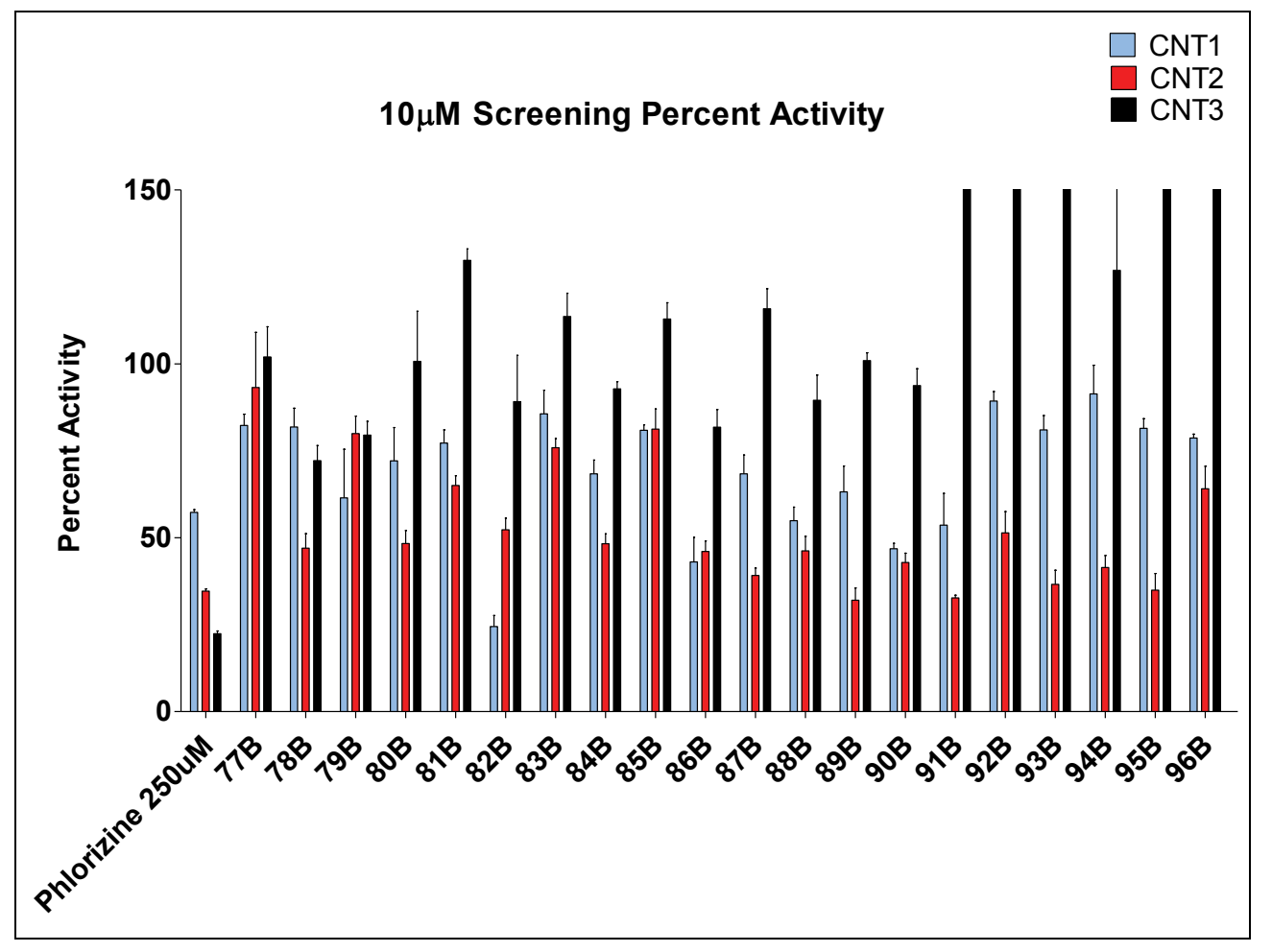

Figure 3-22. 77B-96B screening results

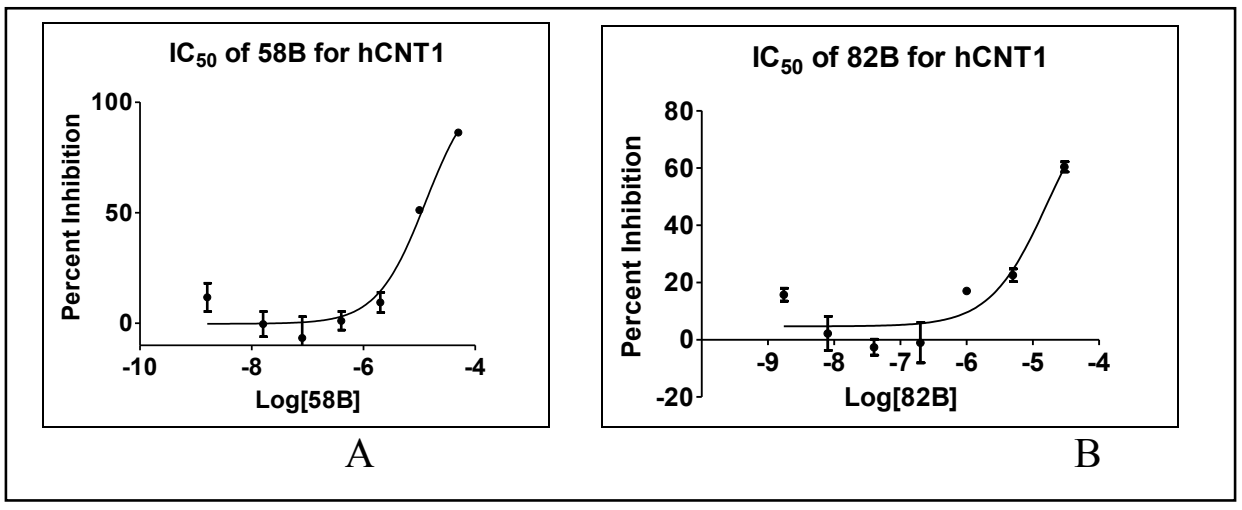

Figure 3-23. Dose response curves for hCNT1 hits

Note: $\mathrm{IC}_{50} \mathrm{~S}^{*}$ : (A) 86.2\% Maximal Inhibition at $50 \mu \mathrm{M}$ (B) 60.4 Maximal Inhibition at 30 $\mu \mathrm{M}$. Interpolated $\mathrm{IC}_{50} \mathrm{~S}(\mathrm{~A}) 12.9 \mu \mathrm{M}$ (B) $16.9 \mu \mathrm{M}$

*95\% CI [M] (A) 5.833e-006 to $2.855 \mathrm{e}-005$ (B) 3.745e-006 to $7.623 \mathrm{e}-005$ 


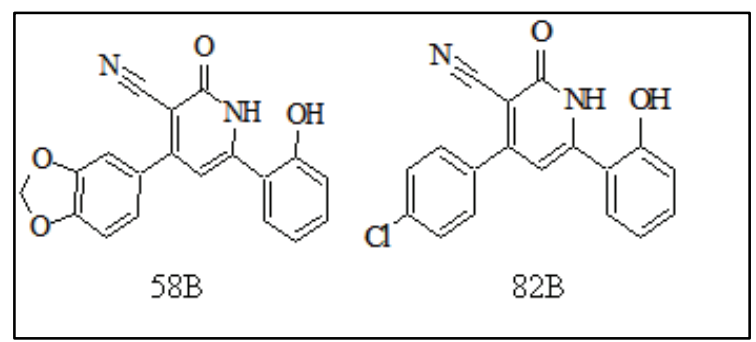

Figure 3-24. hCNT1 hit structures

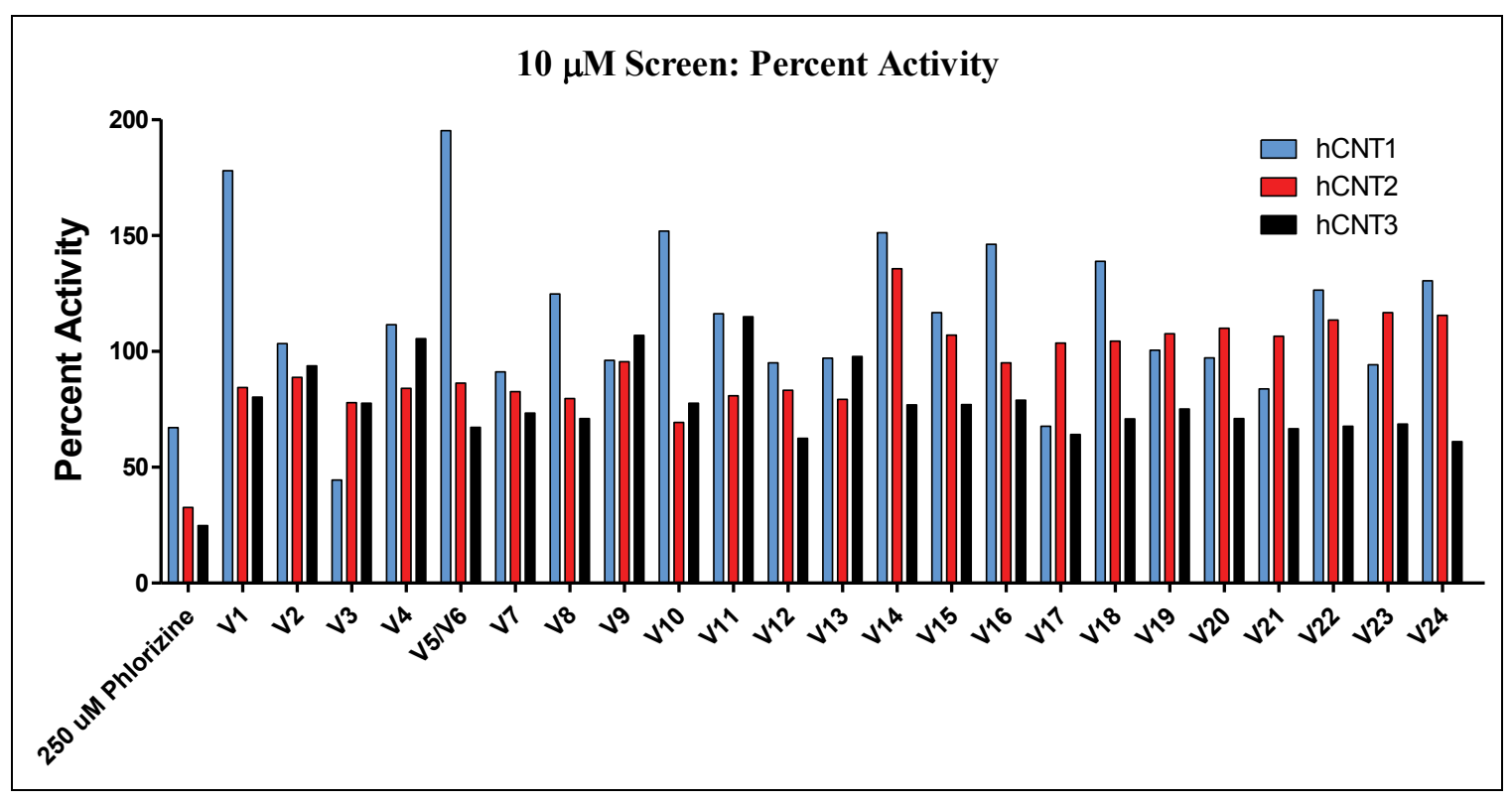

Figure 3-25. V1-V24 screening results

Note: The stocks of V5 and V6 intermixed during shipping of the 96-well plate. As no significant inhibition was noted, V5 and V6 were not reordered for separate testing. Given that there appeared to be potential agonism or activation of the CNT1 transporter, future studies might want to retest separately for confirmation. 


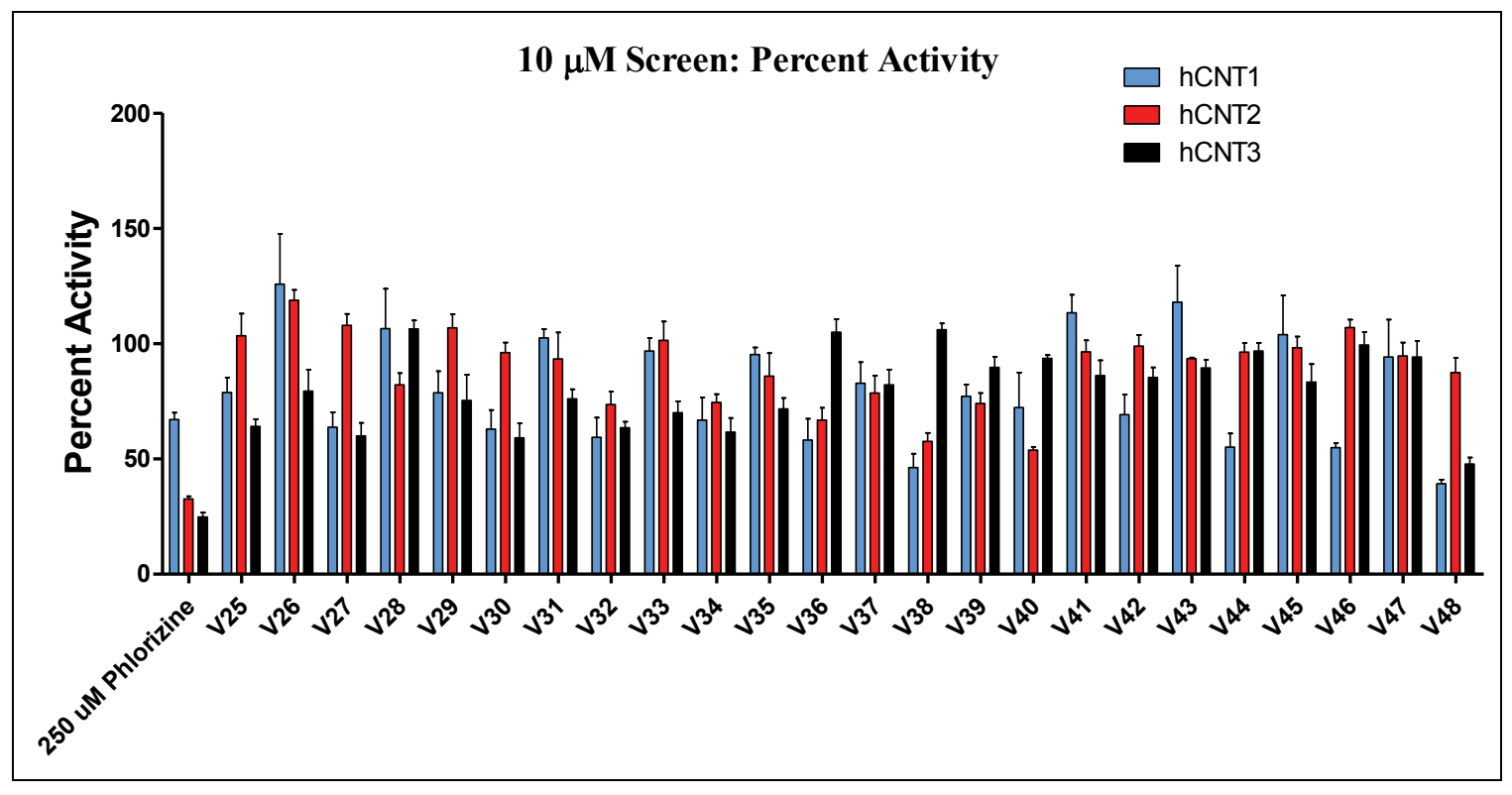

Figure 3-26. V25-V48 screening results

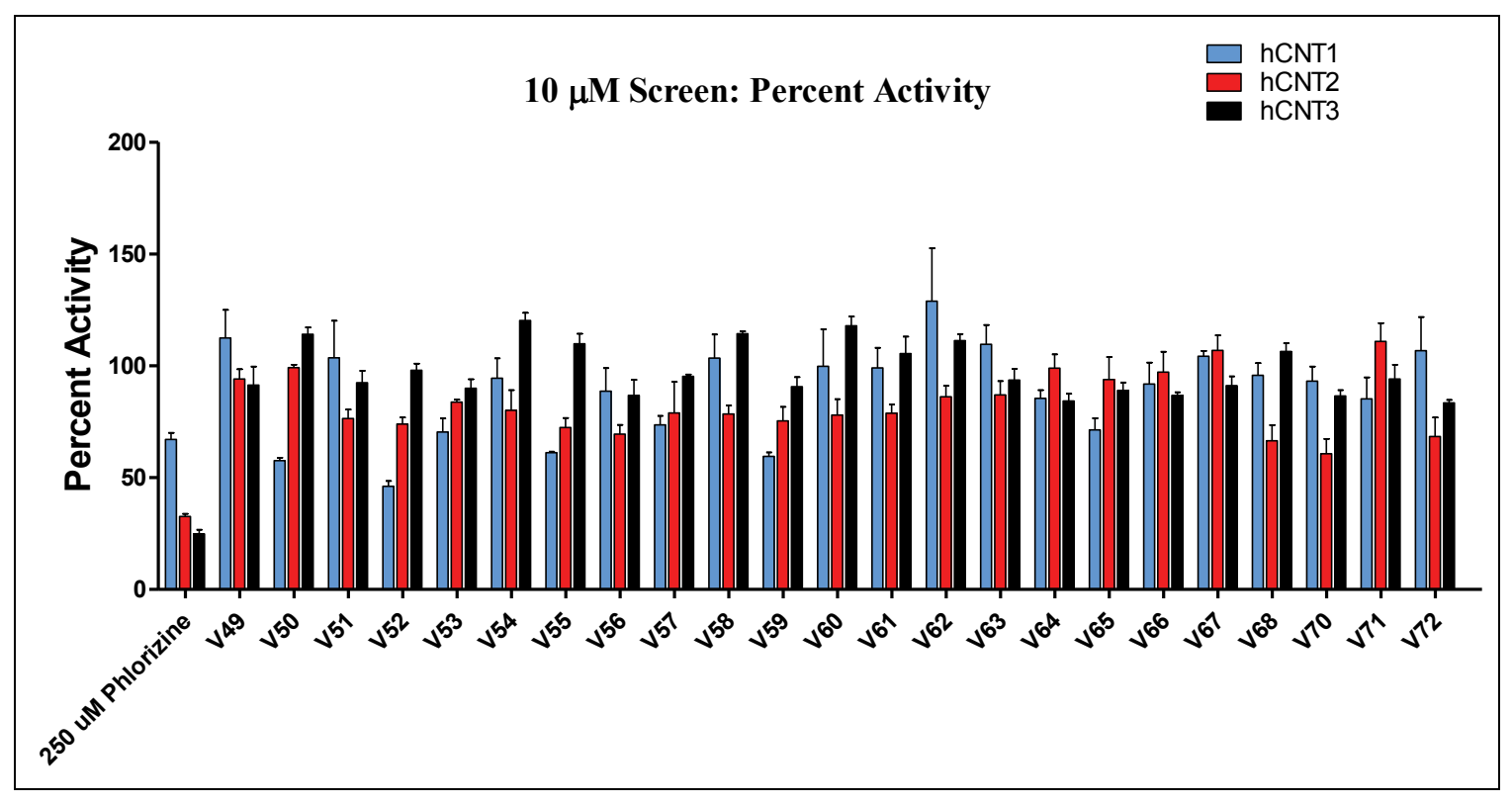

Figure 3-27. V49-V72 screening results 


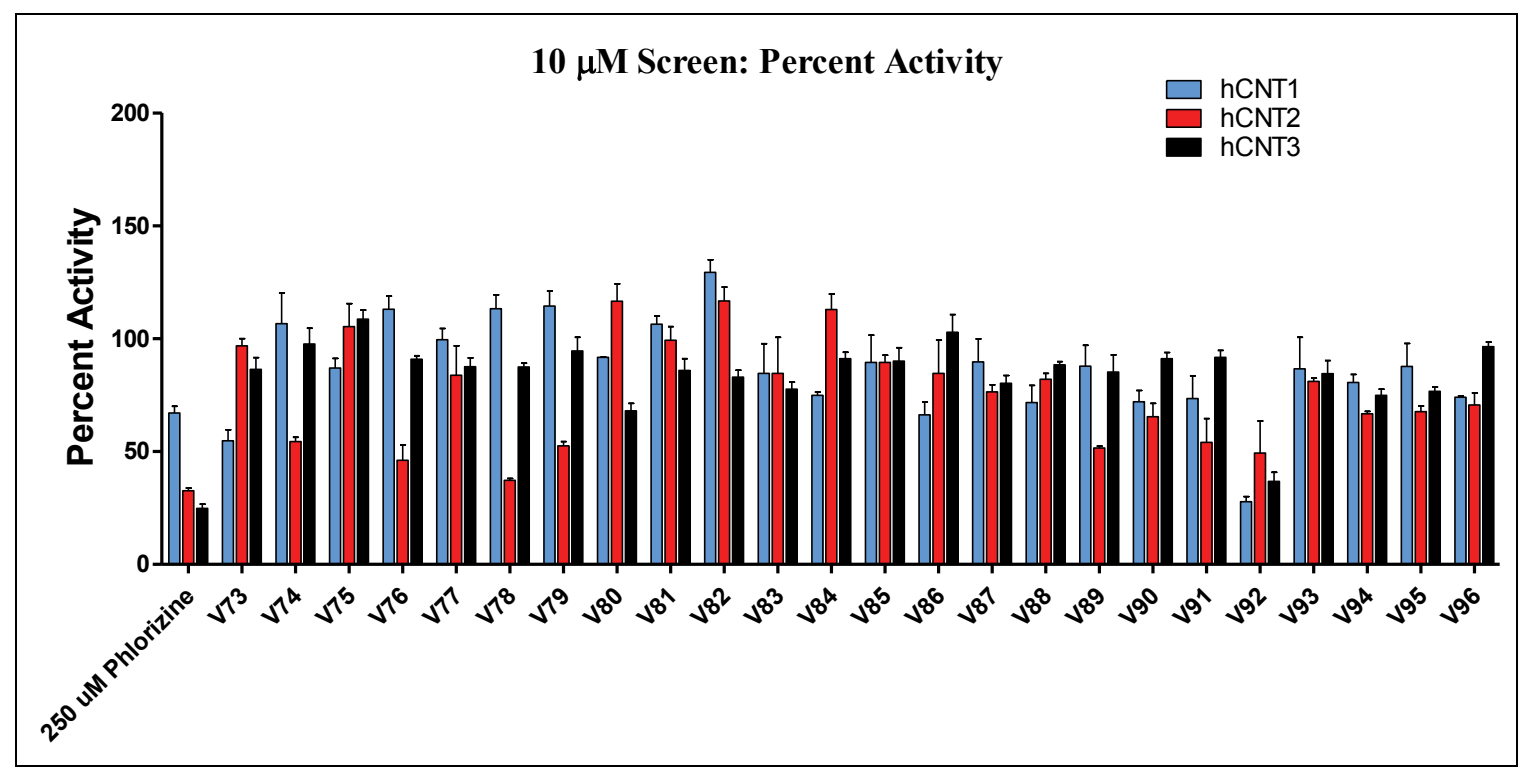

Figure 3-28. V73-V96 screening results

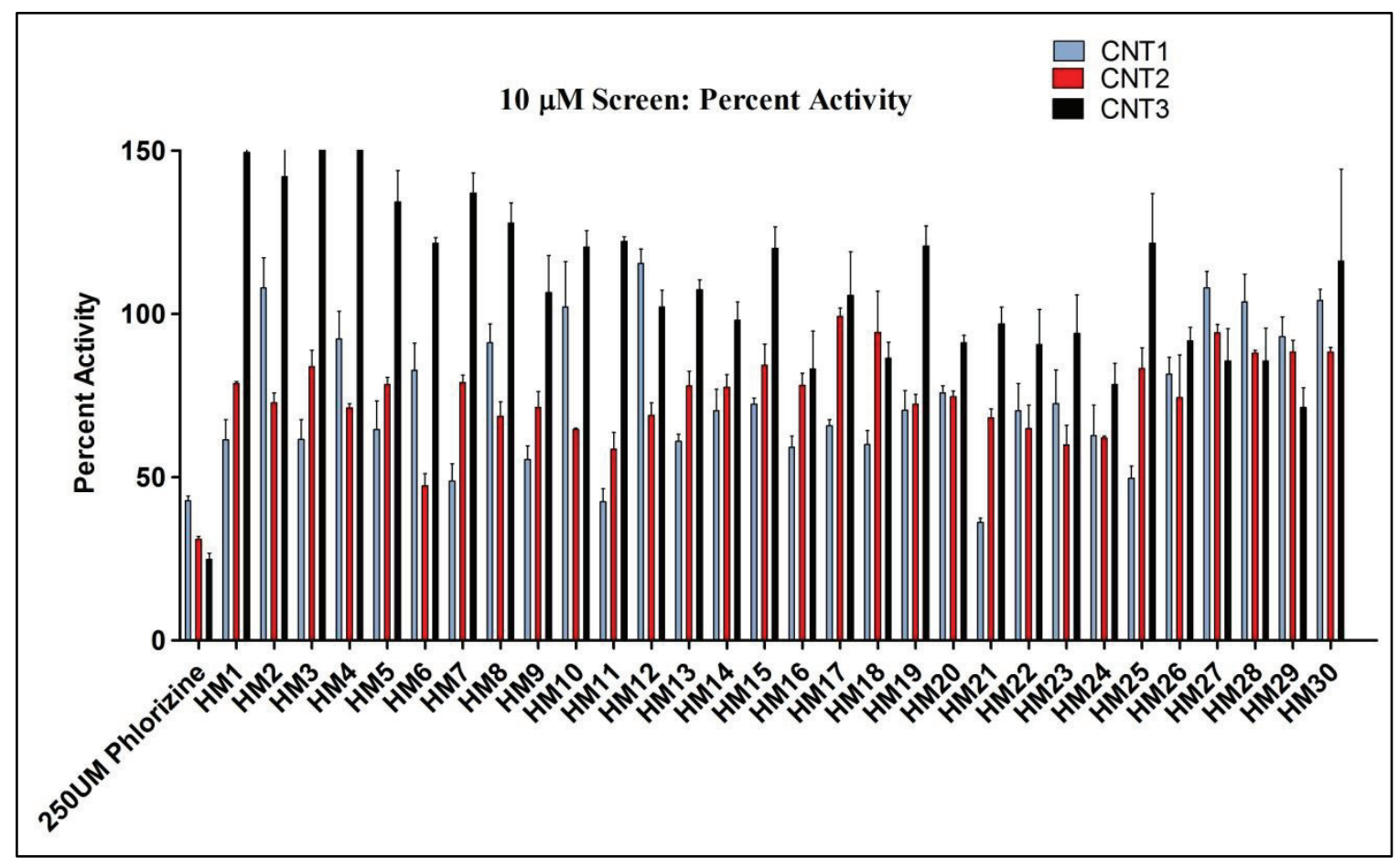

Figure 3-29. Homology model set 1-30 screening results 


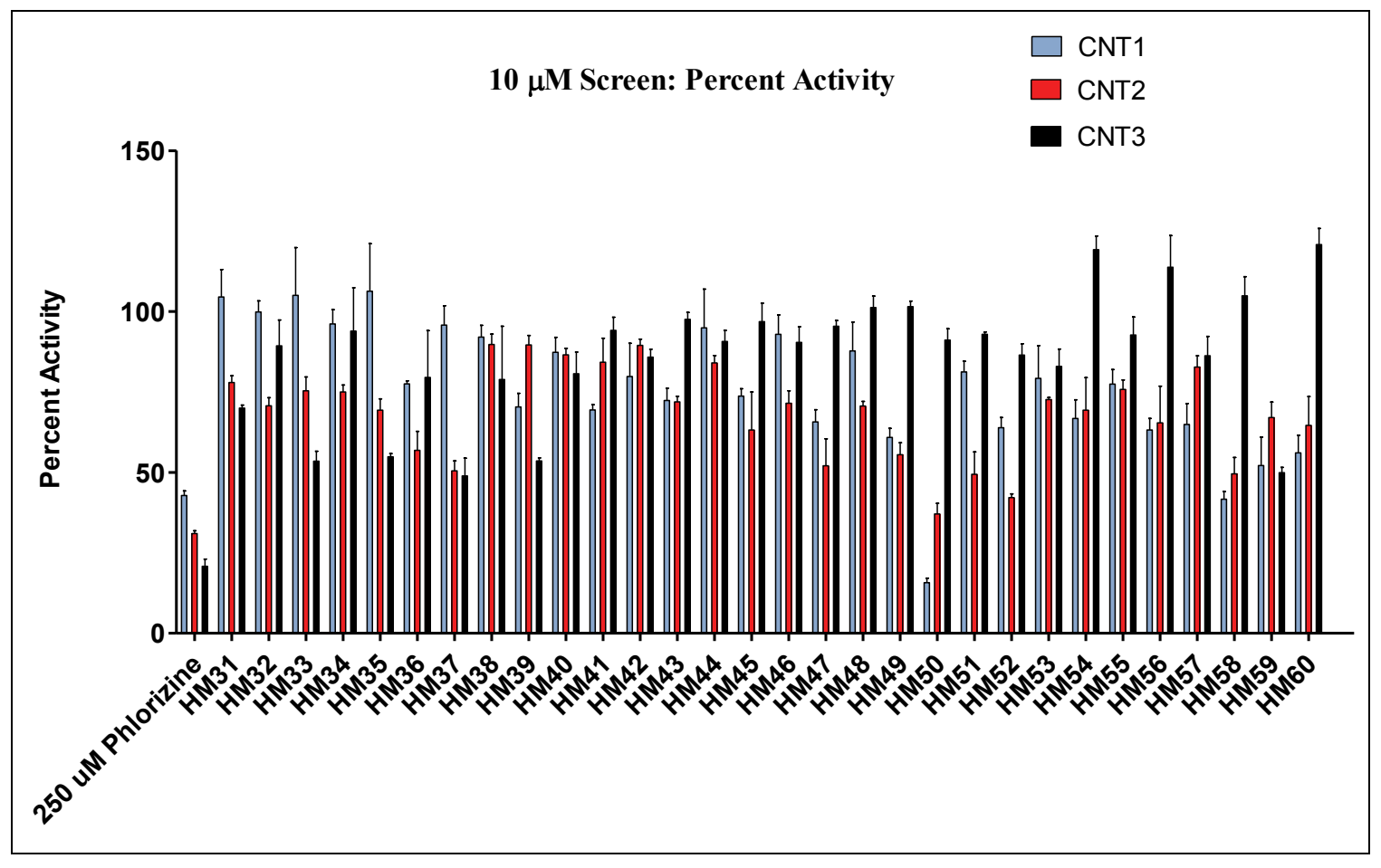

Figure 3-30. Homology model set 31-60 screening results

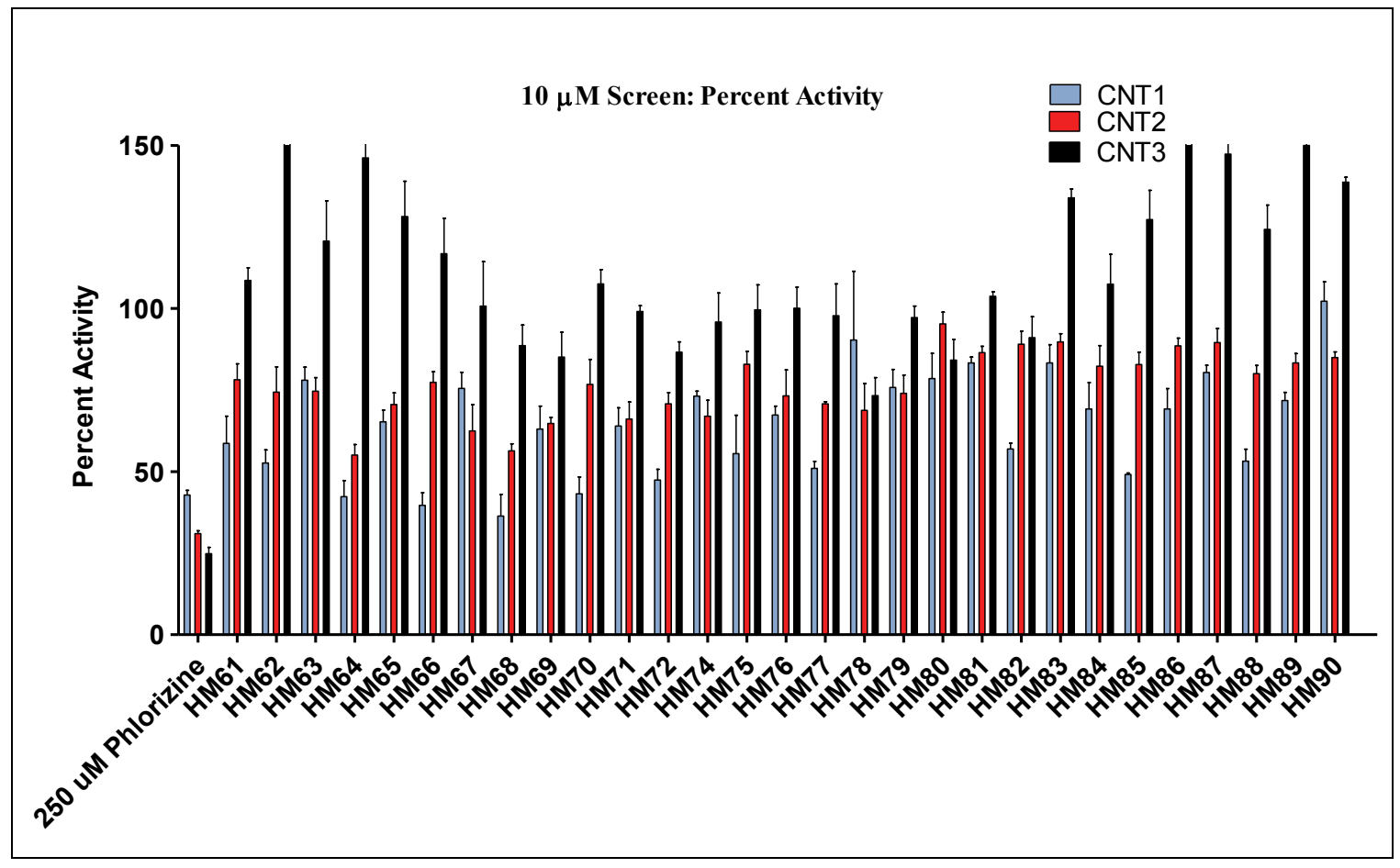

Figure 3-31. Homology model set 61-90 screening results 


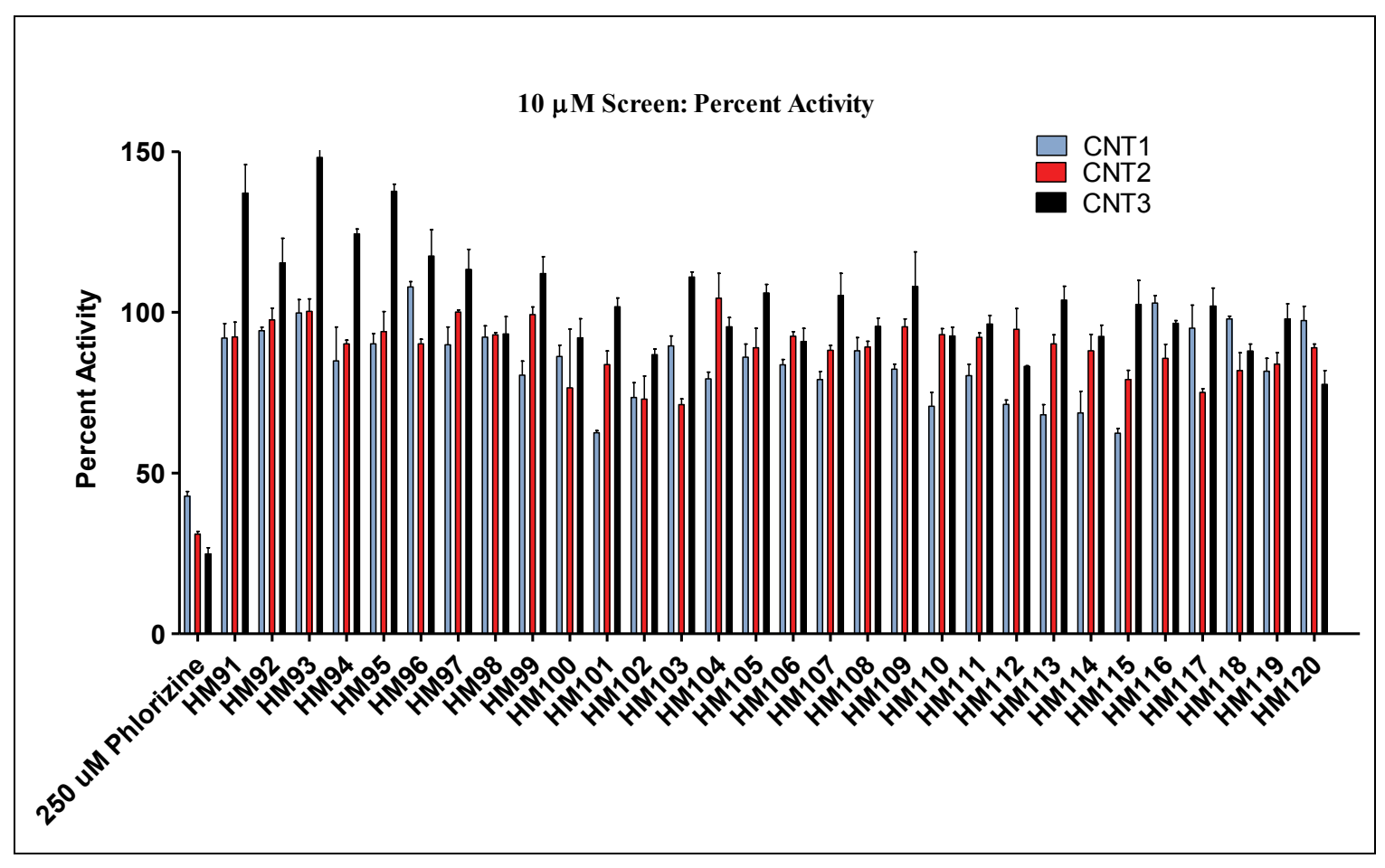

Figure 3-32. Homology model set 91-120 screening results

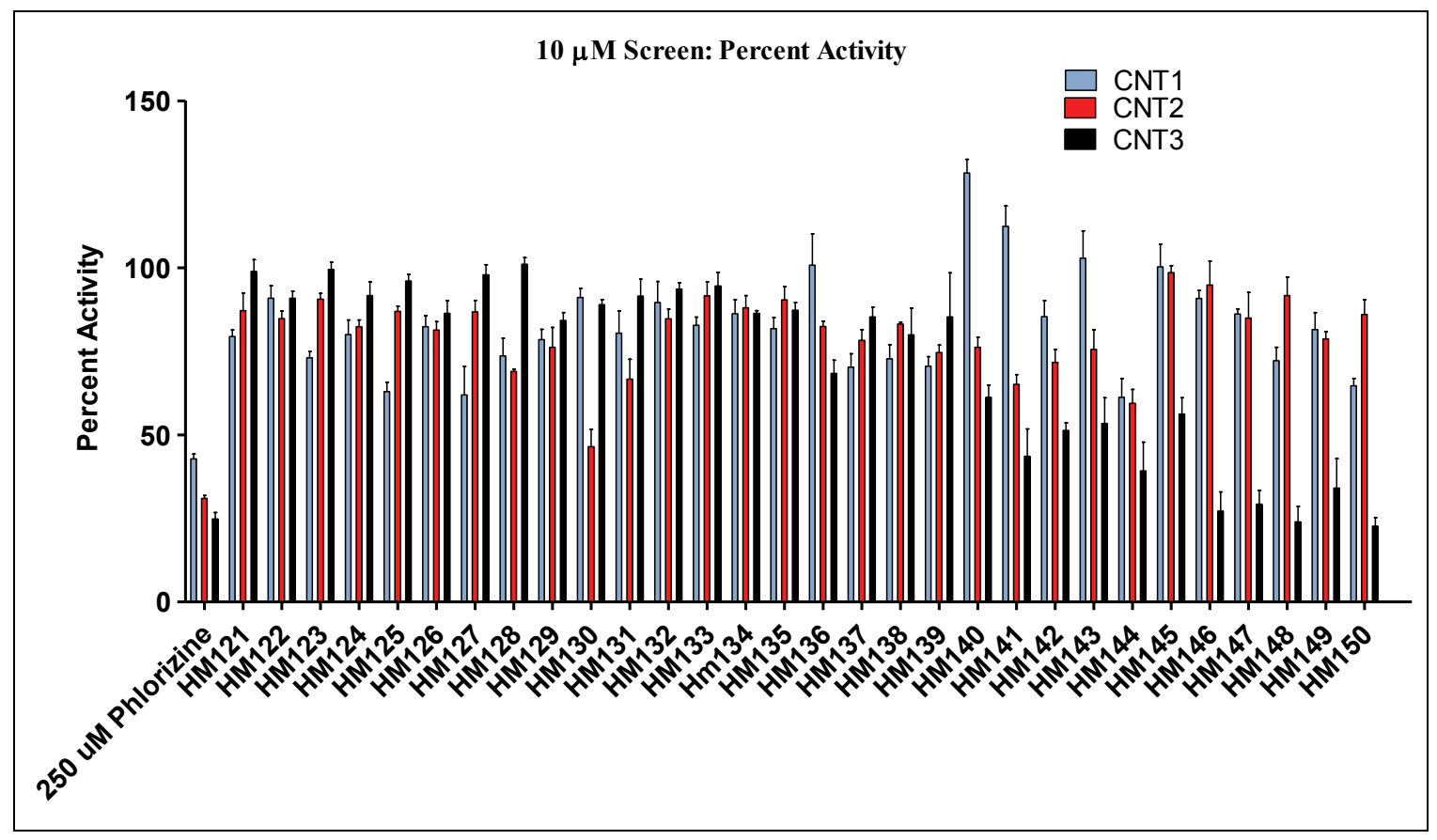

Figure 3-33. Homology model set 121-150 screening results 


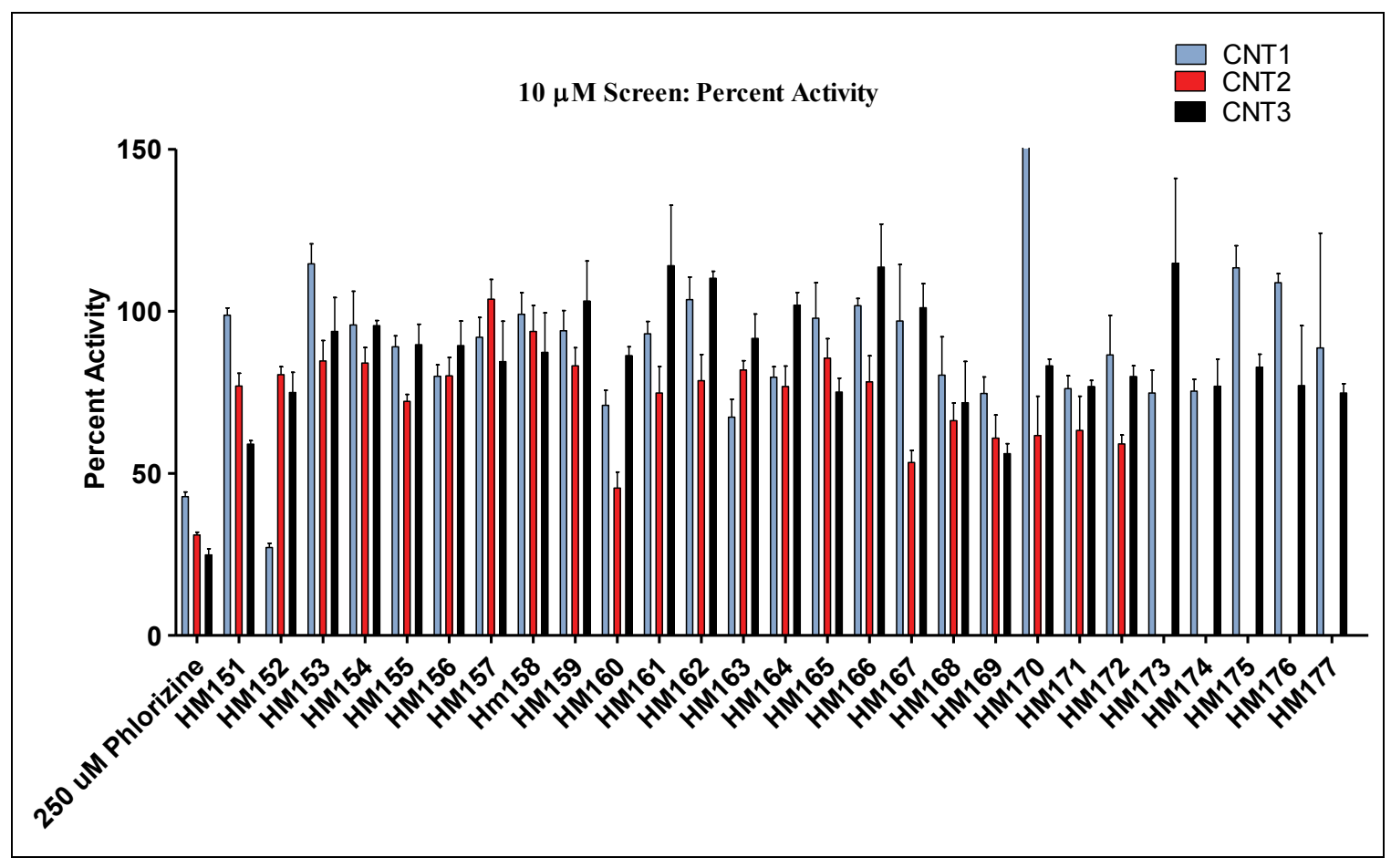

Figure 3-34. Homology model set 151-177 screening results*

Note*: Compounds HM173-177 have not yet been tested against hCNT2 
inhibitory activity disappeared for UC9, and a few others tested. Further kinetic studies, such as examining a time course of range of test compound concentrations at a range of concentrations of tritiated uridine to determine $K_{i}$ s and type of inhibitions (i.e. competitive, non-competitive, etc) and determine if the compound are capable on displacing standard inhibitors should be conducted to better understand the mechanisms of inhibition.

Thus, while the model included pharmacophore elements for nucleoside transporter affinity, it lacked the interactions required for optimal inhibition. As hENT1 was the most easily inhibited of all the transporters, and they all share similarity in their substrates, it was not a surprise that several hENT1 hits were identified.

Of all the hits, UC21 proved most potent, possibly due to the trimethoxy substitution pattern, which has proven to increase activity in dilazep analogues as well as the flavonoids. All the compounds fall in polar surface area and molecular weight ranges for optimization for CNS penetration (Table 3-3). The diverse structures provide an excellent starting point for non-nucleoside potent and selective hENT1 inhibitors as well as potential allosteric modulators. If analogues of hENT4 hits are tested they could serve to expand the structure-activity relationship of non-nucleoside inhibitors of hENT4. All of the compounds require testing for toxicity before further studies are carried out. In addition a panel should be run to confirm selective potency against the desired target and that important enzymes are not inhibited. The drugs listed in Table 1-3 show it is not uncommon for cross reactivity. While cross reactivity does not always rule out a lead compound, it can help to know what sort of side effects and reactions to look for.

In comparing the hCNT1 pharmacophore results and the homology model, they both performed similarly. The pharmacophore hits were not quite as potent, but identified two, whereas the homology model only produced one moderately potent hit. If the $75 \%$ inhibition cut off at $10 \mu \mathrm{M}$ is relaxed to $50 \%$ inhibition, then a number of hits emerge for both models.

In fact, with the $50 \%$ inhibition cut-off, fourteen compounds inhibited hCNT2 at least $50 \%$ at $10 \mu \mathrm{M}$. Only five hit hCNT1, with one hCNT3 hit. Whether this means the model is not truly selective or that hCNT1 and hCNT2 simply share a common pharmacophore remains to be seen. Testing of analogues related to the hits could elucidate the difference. Of particular interest are the compounds that appeared to show a 2- to 4-fold increase in uptake relative to control. While the aim of this study was to identify inhibitors, these results should be noted and explored for future studies (Figure 3-35 and 3-36).

When looking at the 50\% inhibition cut-off, the homology model out-performed the hCNT1 pharmacophore model with fourteen hCNT1 hits. The hCNT1 hit, HM50 had a calculated $\mathrm{IC}_{50}$ of $9.5 \mu \mathrm{M}$ (Figure 3-37), lower than either of the pharmacophore hits. While HM152 appeared to possess activity in the initial screen, it did not show a dose dependent relationship when retested. In addition there were seven weak hCNT2 hits and nine weak hCNT3 hits. This indicated that the model showed selectivity toward hCNT1. However, HM50 proved to be only slightly more potent than 52B and 82B. While 52B 
and $82 \mathrm{~B}$ are pyridone structures, HM50 is a purine analogue (Figure 3-38). It is possible that exploring the pyridone derivatives may elucidate the pharmacophore elements that differentiate a substrate from an inhibitor. While other purine analogues were selected by the model, none showed significant activity in comparison to HM50. 
Table 3-3. hENT1 hit properties

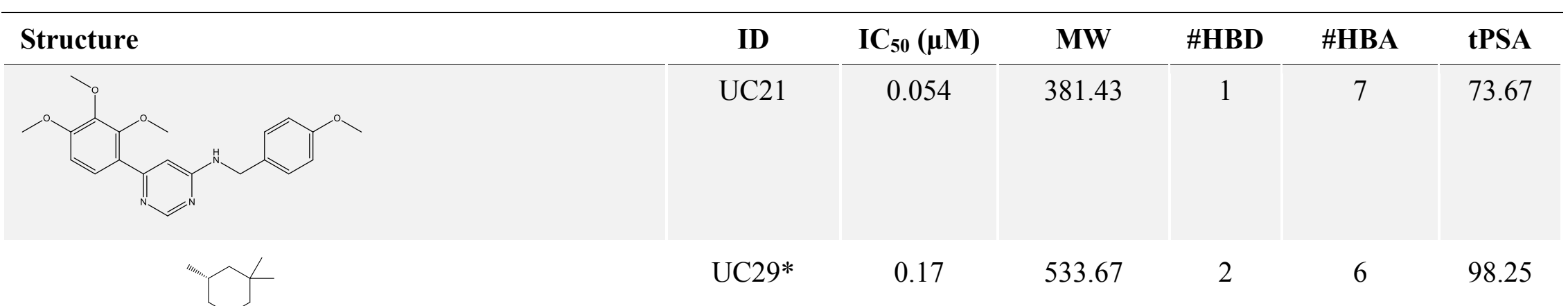

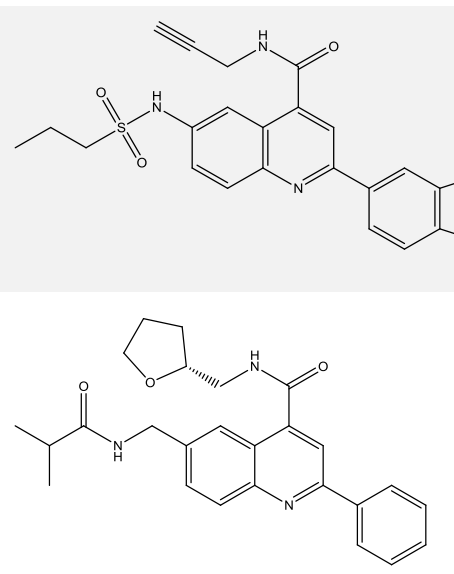

\begin{tabular}{|l|l|l|l|l|l|}
\hline UC33* & 0.31 & 464.590830 & 1 & 4 & 64.68 \\
\hline & & & & \\
\hline & & & & & \\
\hline UC36* & 1.46 & 431.54 & 2 & 4 & 79.79 \\
\hline
\end{tabular}

81 
Table 3-3. Continued

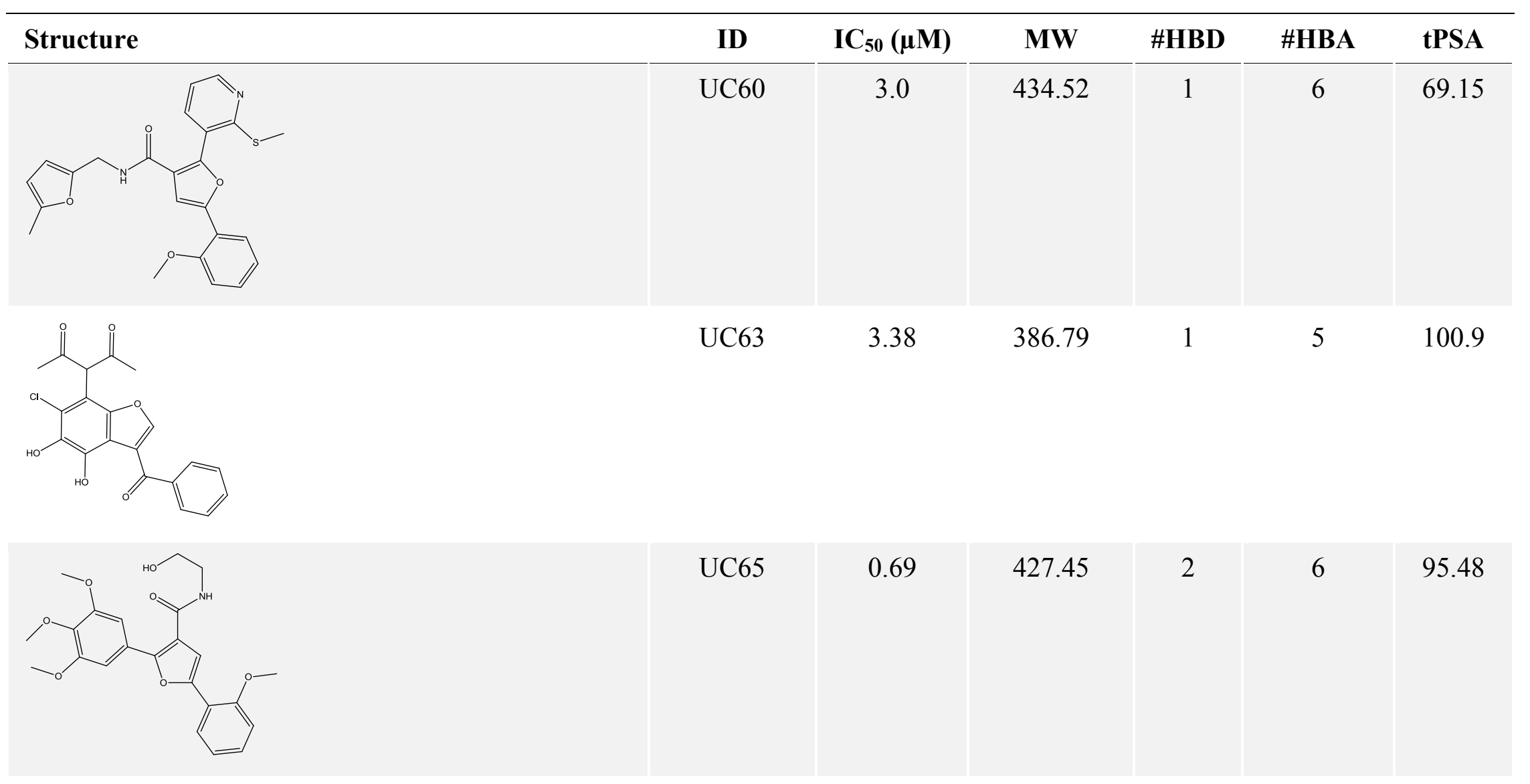


Table 3-3. Continued

\begin{tabular}{|c|c|c|c|c|c|c|}
\hline Structure & ID & $\mathrm{IC}_{50}(\boldsymbol{\mu M})$ & MW & \#HBD & \#HBA & tPSA \\
\hline & UC70 & 0.14 & 288.74 & 1 & 5 & 42.89 \\
\hline & UC86 & 0.75 & 312.37 & 2 & 2 & 46.69 \\
\hline & UC136 & 2.54 & 600.63 & 1 & 7 & 84.86 \\
\hline
\end{tabular}


Table 3-3. Continued

\begin{tabular}{|c|c|c|c|c|c|c|}
\hline Structure & ID & $\mathrm{IC}_{50}(\boldsymbol{\mu M})$ & MW & \#HBD & \#HBA & tPSA \\
\hline & UC137 & 5.27 & 309.346120 & 1 & 4 & 45.98 \\
\hline & UC148 & 0.78 & 469.49 & 2 & 5 & 81.18 \\
\hline & UC173 & 1.86 & 330.36 & 2 & 2 & 44.62 \\
\hline & UC175 & 0.75 & 387.38953 & 1 & 6 & 64.44 \\
\hline
\end{tabular}

Note: *Compound demonstrated mixed activity. 


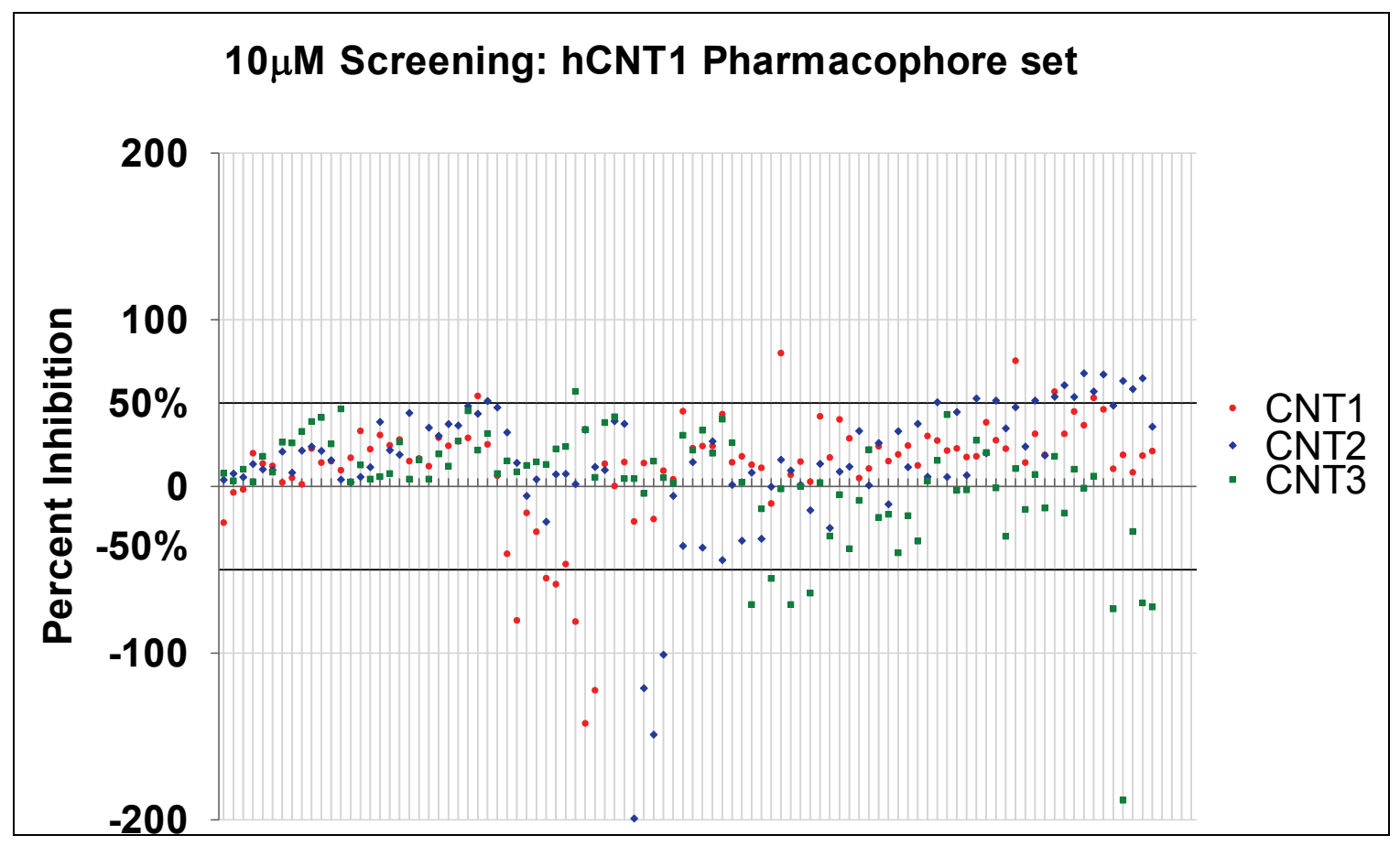

Figure 3-35. hCNT1 pharmacophore model screening results 


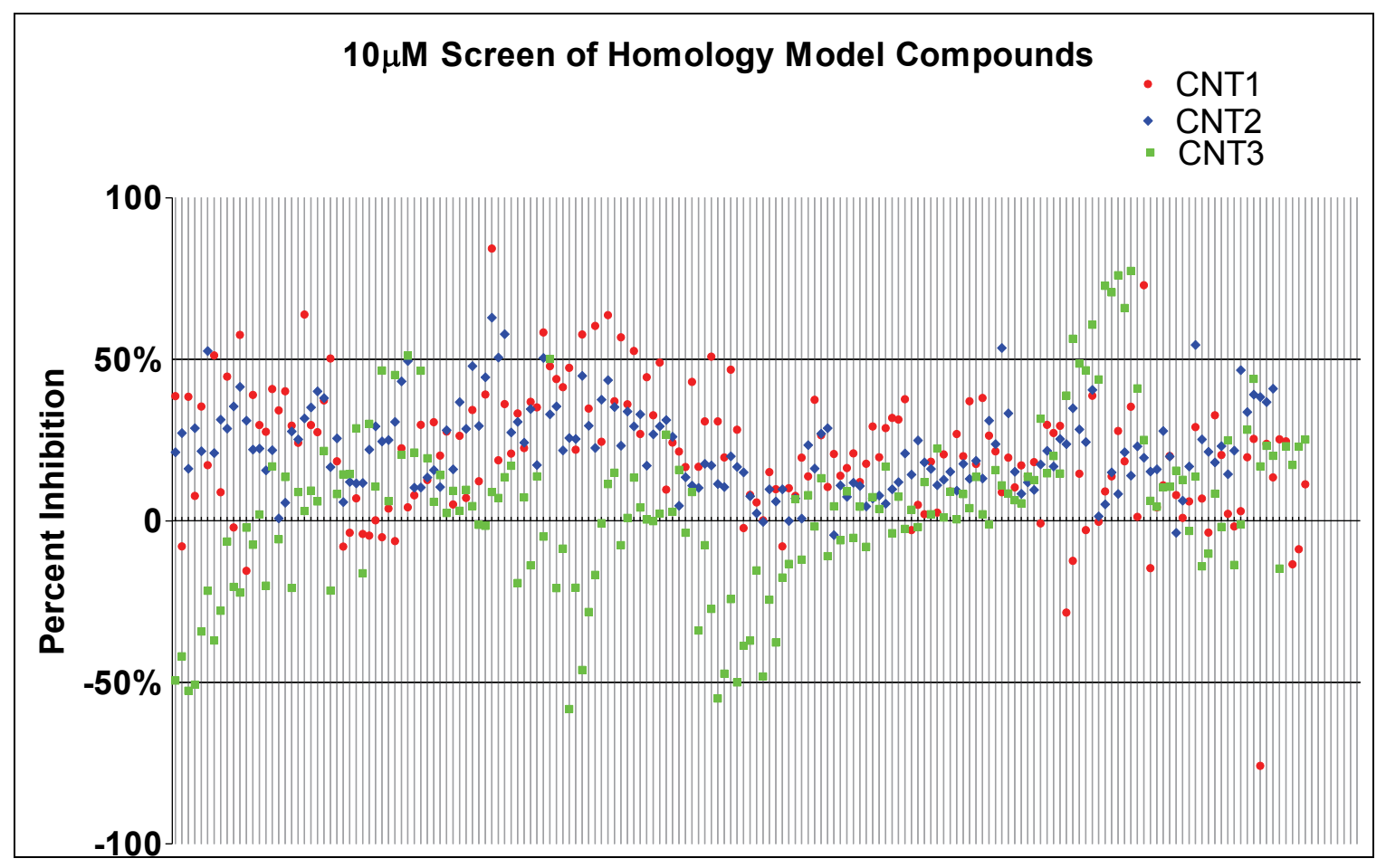

Figure 3-36. Homology model compounds screening results 


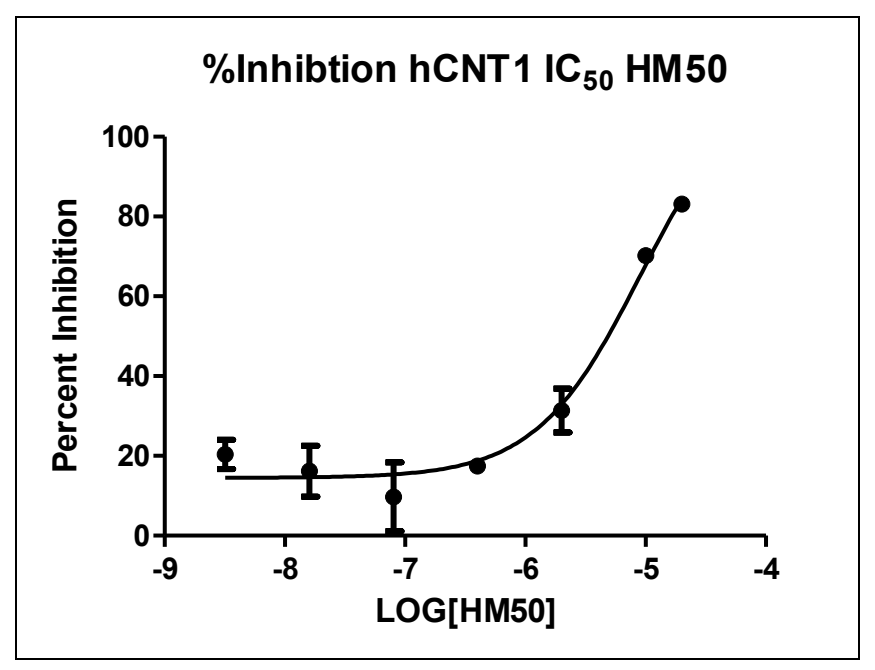

Figure 3-37. Dose dependent curve for HM50

Note: $83.1 \%$ Maximal Inhibition at $20 \mu \mathrm{M}$. Interpolated $\mathrm{IC}_{50}=9.05 \mu \mathrm{M}, 95 \% \mathrm{CI}=$ $3.265 \mathrm{e}-006$ to $2.506 \mathrm{e}-005$

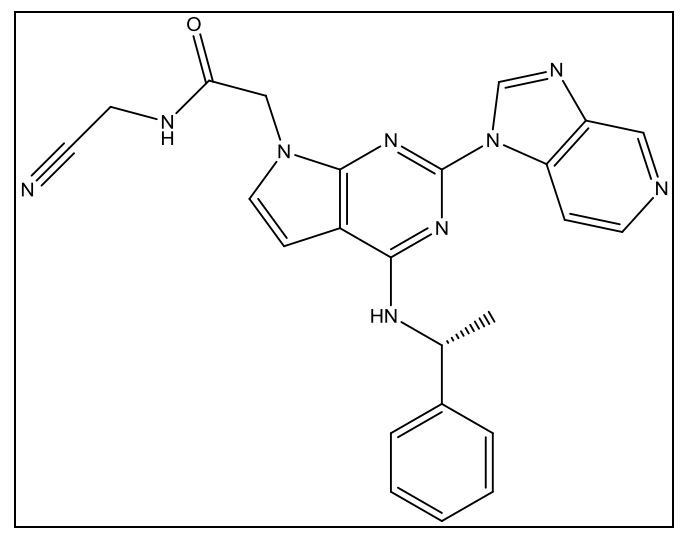

Figure 3-38. HM50 structure 


\section{CHAPTER 4. CHEMISTRY}

\section{Methods}

Thin-layer chromatography (TLC) was conducted on silica gel plates (Analtech) and compounds were visualized by UV light (254 and $365 \mathrm{~nm}$ ). 1D NMR spectra were recorded on a Varian Inova 300, 400, or $500 \mathrm{MHz}$ NMR instrument, as indicated in each spectra in the Appendix, and by using dimethyl sulfoxide- $\mathrm{d}_{6}$ solvent. Flash column chromatography was performed on Fisher silica gel (170-400 mesh). Mass spectra were obtained on a Bruker-HP ESQUIRE ion trap LC/MS- $(n)$ system. All solvents and reagents were purchased from Aldrich or other major chemical companies and used without further purification.

\section{Experimental}

\section{Nucleoside Analogues}

When the project began, it started with the intent of expanding the SAR with regards to the 7-position of the tetrahydroisoquinoline derivatives of NBMPR identified in prior work as potent hENT1 inhibitors. ${ }^{80}$ The work was begun utilizing the published procedure shown in Scheme 4-1.

Several compounds were made following the original procedure. Later, to improve yields, the $\mathrm{CaCO}_{3}$ was replaced with $\mathrm{K}_{2} \mathrm{CO}_{3}$ and then 18-crown- 6 was added in catalytic amount to improve the solubility of the base in the ethanol. Given that the 6chloropurine riboside was readily available, and the amines were of varying cost, it was then used in excess. This route, while acceptable, made purification more complicated as the product and the riboside generally shared very similar solubility properties. Care had to be taken during the reaction not to overheat above $85^{\circ} \mathrm{C}$. When this happened, the heat degraded the sugar, drastically dropping yields. For similar reasons, efforts at a microwave reaction scheme also did poorly.

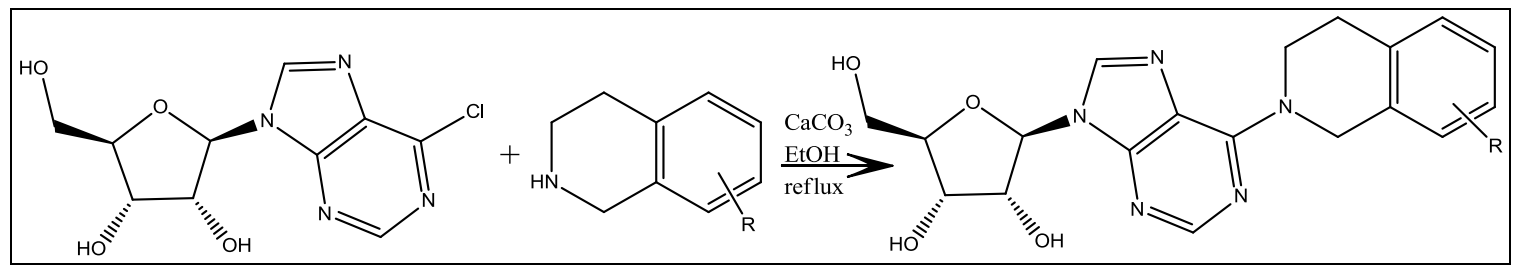

Scheme 4-1. General procedure for constrained NMBPR tetrahydroisoquinoline derivative synthesis 
Due to the highly polar nature of the compounds, they tended to separate poorly with flash chromatography. When possible, recrystallization was the primary means of purification. Mass spectra and NMR spectra can be found for all synthesized compounds in the Appendix (Figures A-18 through A-48).

\section{Original Method}

Structures of synthesized compounds can be found in Figures 4-1 and 4-2.

1) $(2 R, 3 R, 4 S, 5 R)-2-(6-(6-c h l o r o-3,4-d i h y d r o i s o q u i n o l i n-2(1 H)-y l)-9 H-p u r i n-9-$ yl)-5-(hydroxymethyl)tetrahydrofuran-3,4-diol (HCPN01)

$0.88 \mathrm{mmol}$ 6-chloro-1,2,3,4-tetrahydroisoquinoline was added to a round bottom flask with $0.35 \mathrm{mmol} 6$-chloropurine riboside, and $0.70 \mathrm{mmol} \mathrm{CaCO}_{3}$ refluxed in ethanol for 24 hours. It was then purified by flash chromatography using 9:1 ethyl acetate:

methanol. Product was recrystallized from methanol. MS (ESI) $m / z 418(\mathrm{M}+\mathrm{H})_{+}, 440$ $(\mathrm{M}+\mathrm{Na})+\mathrm{C}_{19} \mathrm{H}_{20} \mathrm{ClN}_{5} 0_{4} ;{ }_{1} \mathrm{H}$ NMR (DMSO-d $) 2.95(\mathrm{t}, \mathrm{J}=6 \mathrm{~Hz}, 2 \mathrm{H}), 3.55(\mathrm{~m}, 1 \mathrm{H}), 3.66$ (m, 1H), $3.95(\mathrm{~m}, 1 \mathrm{H}), 4.15(\mathrm{t}, 4 \mathrm{~Hz}, 1 \mathrm{H}), 4.49$ (broad s, overlapping 4.57, s, 3H), (broad overlapping peaks at 5.20, 5.31, $5.42(\mathrm{~s}, 4 \mathrm{H}), 5.94(\mathrm{~d}, \mathrm{~J}=6 \mathrm{~Hz}, 1 \mathrm{H}), 7.26(\mathrm{~m}, 3 \mathrm{H}), 8.29(\mathrm{~s}$, 1H), $8.46(\mathrm{~s}, 1 \mathrm{H})$, Yield 18.8\%.

\section{Modified Methods}

\section{2) 2-(9-((2R,3R,4S,5R)-3,4-dihydroxy-5-(hydroxymethyl)tetrahydrofuran-2-yl)- 9H-purin-6-yl)-1,2,3,4-tetrahydroisoquinoline-5-carbonitrile (HCPN05)}

$1.52 \mathrm{mmol} 6$-chloropurine riboside was added to a round bottom flask with 1.26 mmol 1,2,3,4-tetrahydroisoquinoline-5-carbonitrile, and $1.52 \mathrm{mmol} \mathrm{CaCO}_{3}$ refluxed in ethanol for 24 hours. Product was poorly soluble in ethanol and precipitated out. It was then purified by flash chromatography using ethyl acetate resulting in a white chrystalline solid. MS (ESI) $m / 409(\mathrm{M}+\mathrm{H})+, 431(\mathrm{M}+\mathrm{Na})_{+} ; \mathrm{C}_{20} \mathrm{H}_{20} \mathrm{~N}_{6} 0_{4} ;{ }^{1} \mathrm{H}$ NMR (DMSO- $\left.d_{6}\right) \delta$ $3.08(\mathrm{t}, \mathrm{J}=5.5 \mathrm{~Hz}, 2 \mathrm{H}), 3.56(\mathrm{~m}, 2 \mathrm{H}), 3.66(\mathrm{~m}, 1 \mathrm{H}), 3.96(\mathrm{~d}, \mathrm{~J}=3.5 \mathrm{~Hz}, 1 \mathrm{H}), 4.15,(\mathrm{~m}$, $1 \mathrm{H}), 4.57(\mathrm{~m}, 2 \mathrm{H}), 5.18(\mathrm{~d}, \mathrm{~J}=4.5 \mathrm{~Hz}, 1 \mathrm{H}), 5.28(\mathrm{t}, \mathrm{J}=6 \mathrm{~Hz}, 1 \mathrm{H}), 5.37$ (broad, s, 3-OH) $5.45(\mathrm{~d}, \mathrm{~J}=6.5 \mathrm{~Hz}, 1 \mathrm{H}), 5.94(\mathrm{~d}, \mathrm{~J}=6 \mathrm{~Hz}, 1 \mathrm{H}), 7.42(\mathrm{t}, \mathrm{J}=8 \mathrm{~Hz}, 1 \mathrm{H}), 7.65(\mathrm{~d}, \mathrm{~J}=7.5 \mathrm{~Hz}$, $1 \mathrm{H}), 7.71(\mathrm{~d}, \mathrm{~J}=8 \mathrm{~Hz}, 1 \mathrm{H}), 8.31(\mathrm{~s}, 1 \mathrm{H}), 8.49(\mathrm{~s}, 1 \mathrm{H})$ Yield 7.7\% .

\section{3) $(2 R, 3 R, 4 S, 5 R)-2-(6-(7-b r o m o-3,4-d i h y d r o i s o q u i n o l i n-2(1 H)-y l)-9 H-p u r i n-9-$ yl)-5-(hydroxymethyl)tetrahydrofuran-3,4-diol (HCPN09)}

$1.13 \mathrm{mmol} 6$-chloropurine riboside was added to a round bottom flask with 0.943 mmol 7-bromo-1,2,3,4-tetrahydroisoquinoline, and $1.13 \mathrm{mmol} \mathrm{K}_{2} \mathrm{CO}_{3}$ refluxed in ethanol for 24 hours. Reaction was cooled on ice to precipitate product. Filtrate was rinsed with deionized water to remove the $\mathrm{K}_{2} \mathrm{CO}_{3}$ and dried. Then it was washed with methanol and dissolved in a small volume of ethyl acetate and recrystallized at $4^{\circ} \mathrm{C}$. The TLC showed 
one clean spot. MS (ESI) $m / z 463(\mathrm{M}+\mathrm{H})+; \mathrm{C}_{19} \mathrm{H}_{20} \mathrm{BrN}_{5} 0_{4} ;{ }^{1} \mathrm{H}$ NMR (DMSO-d $d_{6} \delta 2.98$ $(\mathrm{t}, \mathrm{J}=6 \mathrm{~Hz}, 2 \mathrm{H}), 3.34(\mathrm{~d}, \mathrm{~J}=1.5 \mathrm{~Hz}, 2 \mathrm{H}), 3.85(\mathrm{~m}, 2 \mathrm{H}), 4.20(\mathrm{~d}, \mathrm{~J}=2.7 \mathrm{~Hz}, 1 \mathrm{H}), 4.35(\mathrm{~m}$, $1 \mathrm{H}), 4.51$ (broad, s 1-OH), $4.77(\mathrm{t}, \mathrm{J}=6.3 \mathrm{~Hz}, 1 \mathrm{H}), 4.9(\mathrm{~m}, 1 \mathrm{H}), 5.36$ (broad, s 2-OH) $6.0(\mathrm{~d}, 6.3 \mathrm{~Hz}, 1 \mathrm{H}), 7.14(\mathrm{~d}, \mathrm{~J}=8.1 \mathrm{~Hz}, 1 \mathrm{H}), 7.34,(\mathrm{~m}, 1 \mathrm{H}), 7.44(\mathrm{~s}, 1 \mathrm{H}), 8.29$ (d, J= 4.5 $\mathrm{Hz}, 2 \mathrm{H})$ Yield $24.5 \%$.

4) (2R,3S,4R,5R)-2-(hydroxymethyl)-5-(6-(2-methyl-5, 6-dihydropyrido [3,4d]pyrimidin-7(8H)-yl)-9H-purin-9-yl)tetrahydrofuran-3,4-diol (HCPN22)

$0.80 \mathrm{mmol} 6$-chloropurine riboside was added to a round bottom flask with 0.67 mmol 2-methyl-5,6,7,8-tetrahydropyrido[3,4-d]pyrimidine, and $1.60 \mathrm{mmol} \mathrm{K}_{2} \mathrm{CO}_{3}$ refluxed in dry tetrahydrofuran for 24 hours. Riboside and $\mathrm{K}_{2} \mathrm{CO}_{3}$ were filtered from reaction mixture and then the solvent removed by rotovap. The crude product was allowed to dry overnight and then recrystallized in methanol. MS (ESI) $m / z$ 397.9 (M ). $422(\mathrm{M}+\mathrm{Na})_{+;} \mathrm{C}_{18} \mathrm{H}_{21} \mathrm{~N}_{7} 0_{4} ;{ }^{1} \mathrm{H}$ NMR (DMSO-d $) \delta 2.55(\mathrm{~s}, 3 \mathrm{H}), 2.99(\mathrm{t}, \mathrm{J}=5.7 \mathrm{~Hz}, 2 \mathrm{H})$, $3.25(\mathrm{~m}, 1 \mathrm{H}), 3.55(\mathrm{~m}, 1 \mathrm{H}), 3.57(\mathrm{~m}, 1 \mathrm{H}), 3.96(\mathrm{~m}, 1 \mathrm{H}), 4.15(\mathrm{~m}, 1 \mathrm{H}), 4.56(\mathrm{~m} \mathrm{3H}), 5.19$ $(\mathrm{d}, \mathrm{J}=4.8 \mathrm{~Hz}, 1 \mathrm{H}), 5.26(\mathrm{~m}, 2 \mathrm{H}), 5.46(\mathrm{~d}, \mathrm{~J}=6 \mathrm{~Hz}, 1 \mathrm{H}), 5.93(\mathrm{~d}, \mathrm{~J}=6 \mathrm{~Hz}, 1 \mathrm{H}), 8.32(\mathrm{~s}$, 1H), $8.49(\mathrm{~s}, 1 \mathrm{H}), 8.61(\mathrm{~s}, 1 \mathrm{H})$, Yield 20.9\%.

5) $(2 R, 3 R, 4 S, 5 R)-2-(6-(2-c h l o r o-7,8-d i h y d r o-1,6-n a p h t h y r i d i n-6(5 H)-y l)-9 H-$ purin-9-yl)-5-(hydroxymethyl)tetrahydrofuran-3,4-diol (HCPN29)

1.17 mmol 6-chloropurine riboside was added to a round bottom flask with 0.975 mmol 2-chloro-5,6,7,8 tetrahydro-1,6-naphthyridine $\mathrm{HCl}$ salt, and $4.88 \mathrm{mmol} \mathrm{K}_{2} \mathrm{CO}_{3}$ refluxed in 50:50 THF: ethanol for 24 hours. Riboside and $\mathrm{K}_{2} \mathrm{CO}_{3}$ were filtered from reaction mixture and then the solvent removed by rotovap. The crude product was allowed to dry overnight and then recrystallized in methanol. MS (ESI) $m / z(\mathrm{M}+\mathrm{Na})_{+}$; $\mathrm{C}_{18} \mathrm{H}_{19} \mathrm{ClN}_{6} 0_{4} ;{ }^{1} \mathrm{H}$ NMR (DMSO- $\left.d_{6}\right) \delta 3.10(\mathrm{t}, \mathrm{J}=5.7 \mathrm{~Hz}, 2 \mathrm{H}), 3.77(\mathrm{~m}, 1 \mathrm{H}), 3.88$ (m, $1 \mathrm{H}), 4.17(\mathrm{~d}, \mathrm{~J}=2.7 \mathrm{~Hz}, 1 \mathrm{H}), 4.33(\mathrm{~m}, 1 \mathrm{H}), 4.66(\mathrm{~m}, 3 \mathrm{H}), 4.75(\mathrm{t}, \mathrm{J}=5.4 \mathrm{~Hz}, 2 \mathrm{H}), 5.42$ (broad, s, 3H), $5.99(\mathrm{~d}, \mathrm{~J}=5.1 \mathrm{~Hz}, 1 \mathrm{H}), 7.34(\mathrm{~d}, \mathrm{~J}=8.1 \mathrm{~Hz}, 1 \mathrm{H}), 7.73$ (d, J=8.1 Hz, 1H), $8.30(\mathrm{~s}, 1 \mathrm{H}), 8.31(\mathrm{~s}, 1 \mathrm{H})$ Yield $9.5 \%$.

6) $(2 R, 3 R, 4 S, 5 R)-2-(6-(7,8-$ dihydro-1,6-naphthyridin-6(5H)-yl)-9H-purin-9-yl)-5(hydroxymethyl)tetrahydrofuran-3,4-diol (HCPN30)

1.24 mmol 6-chloropurine riboside was added to a round bottom flask with 1.03 mmol 5,6,7,8-tetrahydro-1,6-naphthyridine $\mathrm{HCl}$ salt, and $5.15 \mathrm{mmol} \mathrm{K}_{2} \mathrm{CO}_{3}$ refluxed in 50:50 THF: ethanol for 24 hours. Riboside and $\mathrm{K}_{2} \mathrm{CO}_{3}$ were filtered from reaction mixture and then the solvent removed by rotovap. The crude product was allowed to dry overnight and then recrystallized in methanol. MS (ESI) $\mathrm{m} / z 385(\mathrm{M}+\mathrm{H})_{+}, 407(\mathrm{M}+\mathrm{Na})_{+}$; $\mathrm{C}_{18} \mathrm{H}_{20} \mathrm{~N}_{6} 0_{4} ;{ }^{1} \mathrm{H}$ NMR (DMSO-d $) \delta 3.0(\mathrm{t}, \mathrm{J}=6.3 \mathrm{~Hz}, 2 \mathrm{H}), 3.24(\mathrm{~s}, 1 \mathrm{H}), 3.64(\mathrm{~m}, 3 \mathrm{H})$, $3.95(\mathrm{~m}, 1 \mathrm{H}), 4.15(\mathrm{t}, 3.6 \mathrm{~Hz}, 1 \mathrm{H}), 4.57(\mathrm{t}, \mathrm{J}=5.4 \mathrm{~Hz}, 3 \mathrm{H}), 5.35($ broad, s, 3H), $5.93(\mathrm{~d}, \mathrm{~J}=$ $6 \mathrm{~Hz}, 1 \mathrm{H}), 7.24(\mathrm{~m}, 1 \mathrm{H}), 7.69(\mathrm{~m}, 1 \mathrm{H}), 8.29(\mathrm{~s}, 1 \mathrm{H}), 8.38(\mathrm{~d}, \mathrm{~J}=3.6 \mathrm{~Hz}, 1 \mathrm{H}), 8.46(\mathrm{~s}, 1 \mathrm{H})$ Yield $12.4 \%$. 
7) $(2 R, 3 R, 4 S, 5 R)-2-(6-(5 H-p y r r o l o[3,4-b]$ pyridin-6(7H)-yl)-9H-purin-9-yl)-5(hydroxymethyl)tetrahydrofuran-3,4-diol (HCPN34)

$0.95 \mathrm{mmol} 6$-chloropurine riboside was added to a round bottom flask with 0.79 mmol octahydropyrrolo[1,2-a]pyrazine, and $1.58 \mathrm{mmol} \mathrm{K}_{2} \mathrm{CO}_{3}$ refluxed in THF for 24 hours. Riboside and $\mathrm{K}_{2} \mathrm{CO}_{3}$ were filtered from reaction mixture and then the solvent removed by rotovap. The crude product was allowed to dry overnight and then recrystallized in methanol. MS (ESI) $\mathrm{m} / z 399(\mathrm{M}+\mathrm{Na})_{+} ; \mathrm{C}_{17} \mathrm{H}_{24} \mathrm{~N}_{6} 0_{4} ;{ }^{1} \mathrm{H}$ NMR (DMSO$\left.d_{6}\right) \delta 1.41(\mathrm{~m}, 1 \mathrm{H}), 1.70(\mathrm{~m}, 2 \mathrm{H}), 1.83(\mathrm{~m}, 2 \mathrm{H}), 1.93(\mathrm{~m}, 2 \mathrm{H}), 2.33(\mathrm{~s}, 1 \mathrm{H}), 2.78(\mathrm{~s}, 1 \mathrm{H})$, $2.81(\mathrm{~s}$ broad, $1 \mathrm{H}), 3.03(\mathrm{~m}, 3 \mathrm{H}), 3.11(\mathrm{~s}, 1 \mathrm{H}), 3.54(\mathrm{~m}, 2 \mathrm{H}), 3.95(\mathrm{~m}, 1 \mathrm{H}), 4.15(\mathrm{~m}, 1 \mathrm{H})$, $4.57(\mathrm{~m}, 1 \mathrm{H}), 5.19(\mathrm{~d}, \mathrm{~J}=4.8 \mathrm{~Hz}, 1 \mathrm{H}), 5.33(\mathrm{~m}, 1 \mathrm{H}), 5.46(\mathrm{~d}, \mathrm{~J}=6 \mathrm{~Hz}, 1 \mathrm{H}), 5.91(\mathrm{~d}, \mathrm{~J}=6$ $\mathrm{Hz}, 1 \mathrm{H}), 8.25(\mathrm{~s}, 1 \mathrm{H}), 8.42(\mathrm{~s}, 1 \mathrm{H})$, Yield $2 \%$.

8) (2R,3S,4R,5R)-2-(hydroxymethyl)-5-(6-(6-methoxy-3,4-dihydroisoquinolin2(1H)-yl)-9H-purin-9-yl)tetrahydrofuran-3,4-diol (HCPN58)—not tested

$1.84 \mathrm{mmol}$ 6-chloropurine riboside was added to a microwave tube with 1.53 mmol 6-methoxy-1,2,3,4-tetrahydroisoquinoline, and $3.07 \mathrm{mmol} \mathrm{K}_{2} \mathrm{CO}_{3}$ in ethanol for 24 hours. Riboside and $\mathrm{K}_{2} \mathrm{CO}_{3}$ were filtered from reaction mixture and then the solvent removed by rotovap. The crude product was allowed to dry overnight and then recrystallized in methanol. MS (ESI) $m / z$ 436. $2(\mathrm{M}+\mathrm{Na})_{+} ; \mathrm{C}_{20} \mathrm{H}_{23} \mathrm{~N}_{5} 0_{5} ;{ }^{1} \mathrm{H}$ NMR (DMSO- $\left.d_{6}\right) \delta 2.91(\mathrm{t}, \mathrm{J}=5.6 \mathrm{~Hz}, 2 \mathrm{H}), 3.56(\mathrm{~m}, 3 \mathrm{H}), 3.67(\mathrm{~m}, 2 \mathrm{H}), 3.70(\mathrm{~s}, 1 \mathrm{H}), 3.96(\mathrm{~m}$, $1 \mathrm{H}$ ), 4.14 (m, 1H), 4.45 (broad, s, H), 4.57 (broad s, 1H), 5.18 (broad, s, 2H), 5.33 (broad, s, 1H), 5.46 (broad,s, 1H), $5.92(\mathrm{~d}, \mathrm{~J}=6 \mathrm{~Hz}, 1 \mathrm{H}), 6.77(\mathrm{~m}, 2 \mathrm{H}), 7.16(\mathrm{~d}, \mathrm{~J}=8.4 \mathrm{~Hz}$, $1 \mathrm{H}), 8.28(\mathrm{~s}, 1 \mathrm{H}), 8.44(\mathrm{~s}, 1 \mathrm{H})$ Yield 56\%.

9) $(2 R, 3 S, 4 R, 5 R)-2-($ hydroxymethyl)-5-(6-(5-(trifluoromethyl)-3,4dihydroisoquinolin-2(1H)-yl)-9H-purin-9-yl)tetrahydrofuran-3,4-diol (HCPN69)—not tested

$0.51 \mathrm{mmol}$ 6-chloropurine riboside was added to a microwave tube with 0.42 mmol 5-(trifluoromethyl)-1,2,3,4-tetrahydroisoquinoline hydrochloride salt, $0.92 \mathrm{mmol}$ $\mathrm{K}_{2} \mathrm{CO}_{3}$ and a catalytic amount of 18-crown-6 in ethanol for 24 hours. Riboside and $\mathrm{K}_{2} \mathrm{CO}_{3}$ were filtered from reaction mixture and then the solvent removed by rotovap. The crude product was allowed to dry overnight and then recrystallized in methanol. MS (ESI) $m / z(\mathrm{M}+\mathrm{Na})_{+} \mathrm{C}_{20} \mathrm{H}_{20} \mathrm{~F}_{3} \mathrm{~N}_{5} 0_{4} ;{ }^{1} \mathrm{H}$ NMR (DMSO- $\left.d_{6}\right) \delta 3.05(\mathrm{t}, \mathrm{J}=6 \mathrm{~Hz}, 2 \mathrm{H}), 3.55$ $(\mathrm{m}, 2 \mathrm{H}), 3.67(\mathrm{~m}, 2 \mathrm{H}), 3.95(\mathrm{~m}, 1 \mathrm{H}), 4.14(\mathrm{~m}, 1 \mathrm{H}), 4.55(\mathrm{t}, \mathrm{J}=5.2 \mathrm{~Hz}, 2 \mathrm{H}), 5.21$ (broad, s, 2H), 5.24 (broad, s, 2H), $5.93(\mathrm{t}, \mathrm{J}=4.8 \mathrm{~Hz}, 1 \mathrm{H}), 7.40(\mathrm{t}, \mathrm{J}=7.6 \mathrm{~Hz}, 1 \mathrm{H}), 7.50(\mathrm{~d}, \mathrm{~J}=7.6$ $\mathrm{Hz}, 1 \mathrm{H}), 7.55(\mathrm{~d}, \mathrm{~J}=7.6 \mathrm{~Hz}, 1 \mathrm{H}), 8.32(\mathrm{~s}, 1 \mathrm{H}), 8.47(\mathrm{~s}, 1 \mathrm{H})$, Yield 41.9\%.

10) 2-(9-((2R,3R, 4S, 5R)-3,4-dihydroxy-5-(hydroxymethyl)tetrahydrofuran-2-yl)9H-purin-6-yl)-1,2,3,4-tetrahydroisoquinoline-5-carboxylate (HCPN78)not tested

$0.87 \mathrm{mmol} 6$-chloropurine riboside was added to a microwave tube with $1.2 \mathrm{mmol}$ 1,2,3,4-tetrahydroisoquinoline-5-carboxylate hydrochloride salt, and $4.2 \mathrm{mmol} \mathrm{K}_{2} \mathrm{CO}_{3}$ in 
ethanol for 24 hours. Riboside and $\mathrm{K}_{2} \mathrm{CO}_{3}$ were filtered from reaction mixture and then the solvent removed by rotovap. The crude product was allowed to dry overnight and then recrystallized in methanol. MS (ESI) $m / z(\mathrm{M}+\mathrm{Na})_{+} ; \mathrm{C}_{20} \mathrm{H}_{21} \mathrm{~N}_{5} \mathrm{O}_{6} ;{ }^{1} \mathrm{H}$ NMR (DMSOd6) $\delta 2.32(\mathrm{~s}, 1 \mathrm{H}), 3.21(\mathrm{~m}, 4 \mathrm{H}), 3.54(\mathrm{~m}, 3 \mathrm{H}), 3.95(\mathrm{~m}, 1 \mathrm{H}), 4.15(\mathrm{~m}, 1 \mathrm{H}), 4.56(\mathrm{~m}, 1 \mathrm{H})$, $5.21(\mathrm{~m}, 2 \mathrm{H}), 5.35(\mathrm{~m}, 2 \mathrm{H}), 5.51(\mathrm{~m}, 1 \mathrm{H}), 5.92(\mathrm{~d}, \mathrm{~J}=5.84 \mathrm{~Hz}, 1 \mathrm{H}), 7.21(\mathrm{~m}, 1 \mathrm{H}), 7.47$ $(\mathrm{m}, 1 \mathrm{H}), 8.21(\mathrm{~s}, 1 \mathrm{H}), 8.44(\mathrm{~s}, 1 \mathrm{H})$ Yield $11.5 \%$.

\section{Pyridone Analogues}

Literature searches provided a number of established methods for synthesizing pyridones, depending on the substitution pattern. The first method used (Scheme 4-2) involved reaction of a substituted diketone with 2-cyanoacetimide with an acidic aqueous workup. While Pyr2 (Figure 4-3) was successfully produced with this method, depending on $\mathrm{R}^{1}$ and $\mathrm{R}^{2}$ and the electron donating or withdrawing capacity of those substituents, more than one structural isomer could result. Two other compounds attempted with this method resulted in what was suspected to be a flavone-like bicyclic structure instead of the desired products. Even for the compounds synthesized in this manner, it was required to synthesize the diketones. Thus, the synthetic scheme resulted in several purification steps, thereby decreasing yields to less than $20 \%$.

To synthesize the diketones, 2'-hydroxyacetophenone was reacted with an aromatic acid chloride, followed by Baeyer-Villiger rearrangement. ${ }^{81}$ Heteroaromatic compounds in particular proved less reactive.

An alternate one-pot synthesis method was adopted after further search into the literature (Scheme 4-3). ${ }^{82}$ The initial test reaction, which was conducted neat, worked, but yield was poor and required hours rather than the mere 10-15 minutes reported in the reference. Dissolving all reactants in propanol and refluxing at $110^{\circ} \mathrm{C}$ greatly improved the reaction's efficiency, but heteroaromatics remained less reactive in comparison to aromatic carbocyclics.

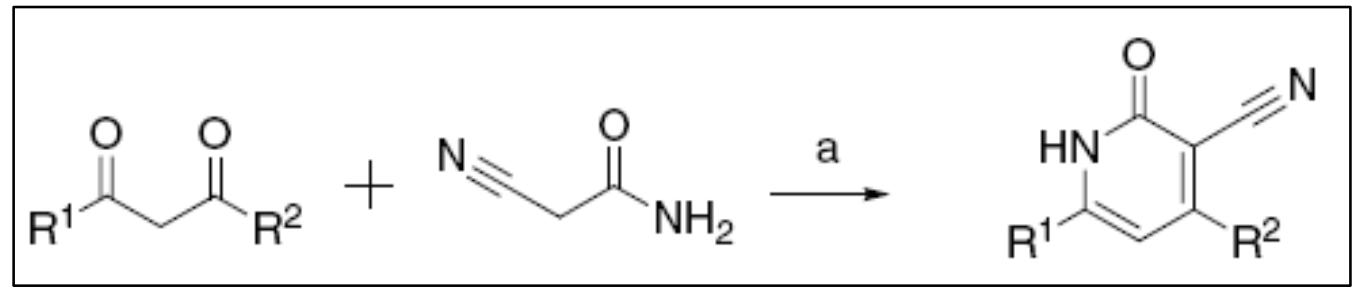

Scheme 4-2. Synthetic procedure for diketone and 2-cyanoacetimed synthesis of substituted pyridones

Note: Reagents and conditions: (a) toluene, DBU, reflux, $95^{\circ} \mathrm{C}$ 


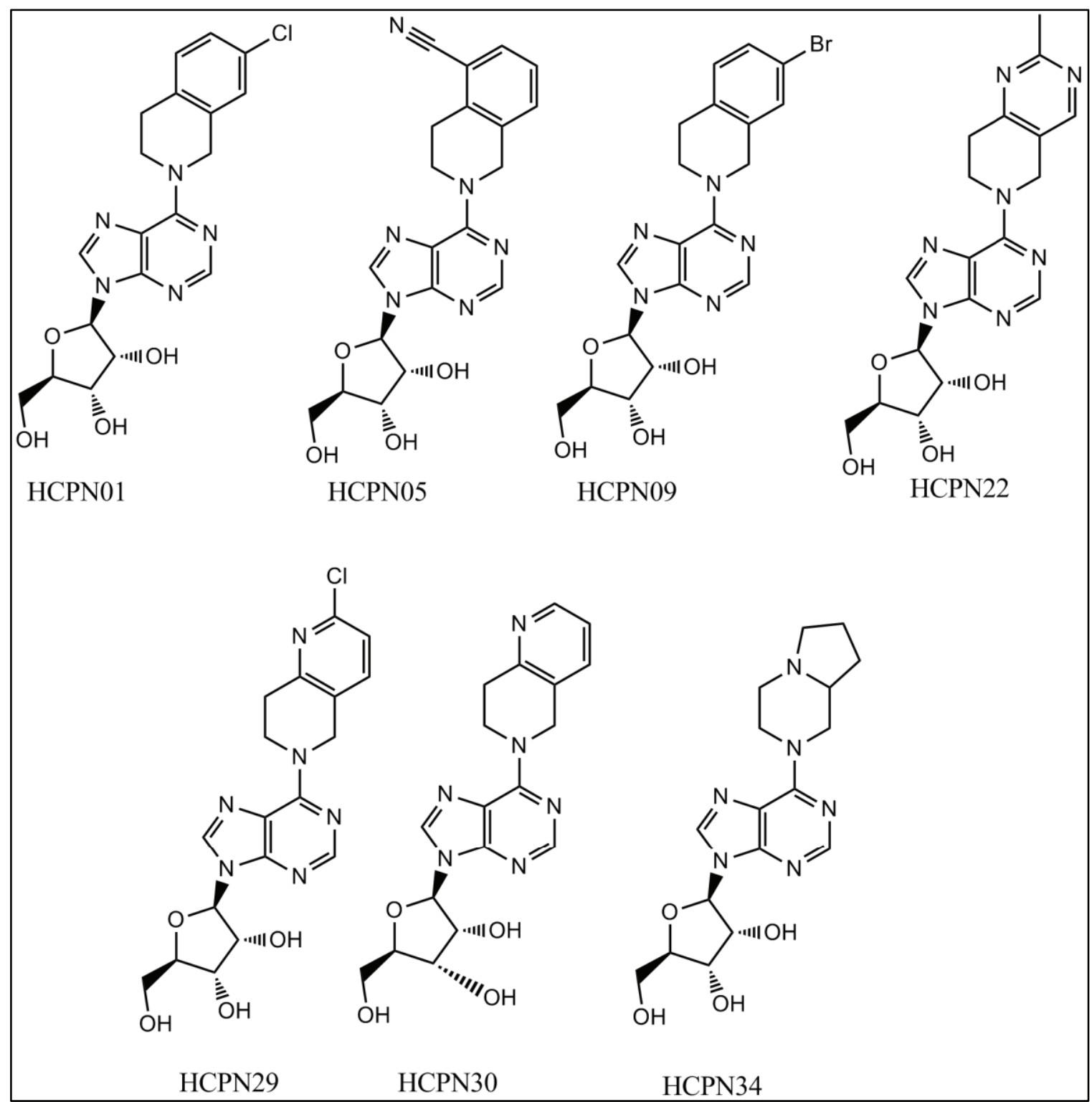

Figure 4-1. Synthesized and tested nucleoside analogues 


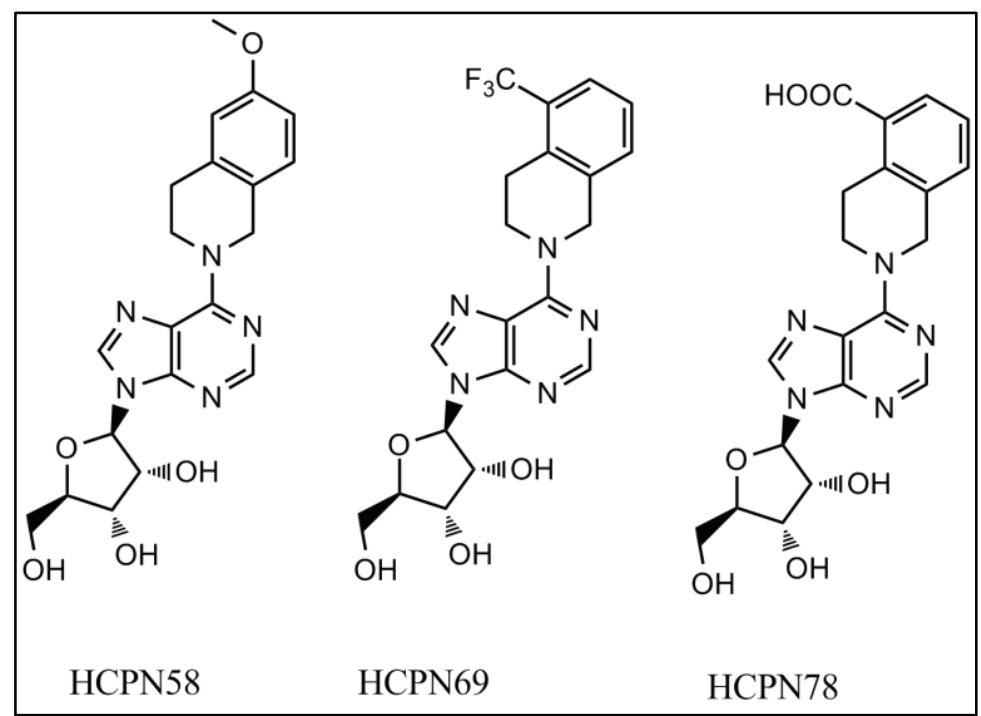

Figure 4-2. Synthesized and untested nucleoside analogues

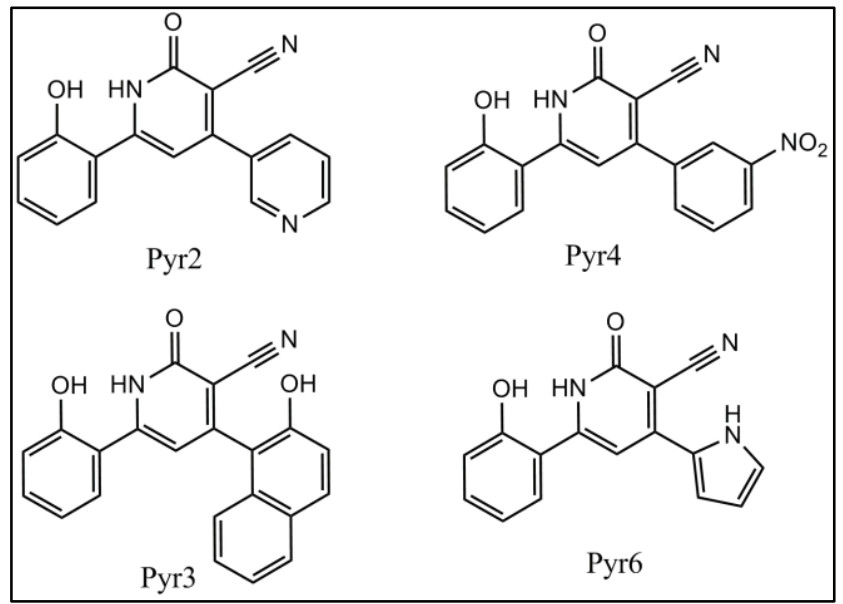

Figure 4-3. Synthesized and tested pyridones 


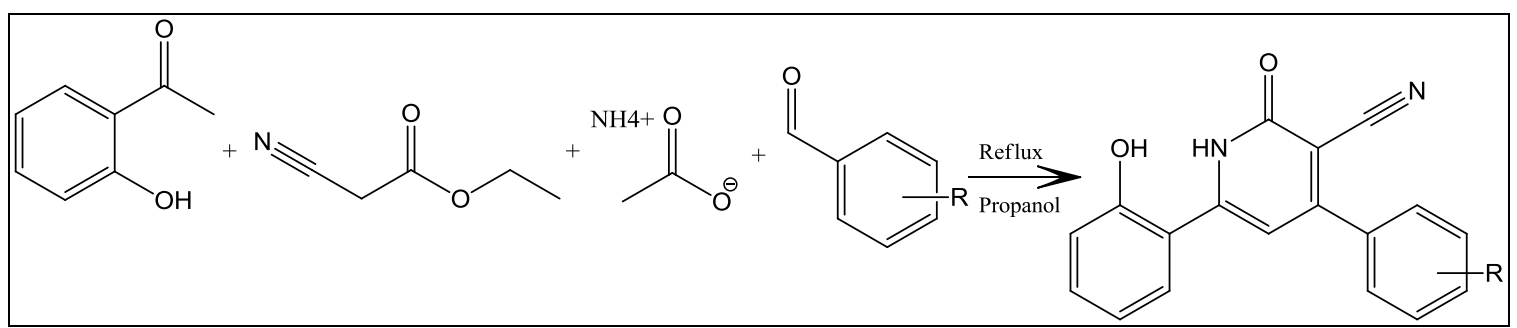

Scheme 4-3. One-pot synthesis of pyridones

Note: Reagents and conditions: propanol, $110^{\circ} \mathrm{C}, 24 \mathrm{hrs}$. 


\section{Method One}

First 2-hydroxyacetophenone was reacted in a 1:1 molar ratio with the appropriate aryl acid chloride at room temperature overnight or until reaction was judged by TLC to be complete.

\section{1) 6'-(2-hydroxyphenyl)-2'-oxo-1',2'-dihydro-[3,4'-bipyridine]-3'-carbonitrile}

(Pyr2)

$10 \mathrm{mmol}$ of 2-hydroxyacetophenone was reacted with $1.44 \mathrm{mmol}$ of nicotinic carbonyl chloride $\mathrm{HCl}$ salt at room temperature overnight in $25 \mathrm{ml}$ of pyridine. Reaction was worked up by adding water and then adjusting the $\mathrm{pH}$ to approximately 4 . The precipitated diketone intermediate, bright yellow crystals, was filtered and dried overnight (14\% yield). Then $0.5 \mathrm{mmol}$ reaction intermediate was reacted with $0.5 \mathrm{mmol}$ 2-cyanoacetamide in toluene and $0.025 \mathrm{mmol}$ DBU (1,8-Diazabicycloundec-7-ene). $21 \mathrm{mg}$ of product obtained through recrystallization in methanol. MS (ESI) $\mathrm{m} / \mathrm{z} 289$ (M$\mathrm{H})-; \mathrm{C}_{17} \mathrm{H}_{11} \mathrm{~N}_{3} 0_{2} ;{ }^{1} \mathrm{H}$ NMR (DMSO- $\left.d_{6}\right) \delta 7.22(\mathrm{~s}, 1 \mathrm{H}), 7.65(\mathrm{~m}, 2 \mathrm{H}), 7.84(\mathrm{~m}, 2 \mathrm{H}), 8.07$ (d, $\mathrm{J}=8 \mathrm{~Hz}, 1 \mathrm{H}), 8.52(\mathrm{~m}, 1 \mathrm{H}), 8.79(\mathrm{~m}, 1 \mathrm{H}), 9.33(\mathrm{~m}, 1 \mathrm{H})$, exchangeable protons not visible, $(<1 \%$ yield $)$.

\section{Method Two}

12) 4-(2-hydroxynaphthalen-1-yl)-6-(2-hydroxyphenyl)-2-oxo-1,2dihydropyridine-3-carbonitrile (Pyr3)

$5.8 \mathrm{mmol}$ of 2-hydroxy-1-naphthaldehyde was reacted with $5.8 \mathrm{mmol} \mathrm{2'-}$ hydroxyacetophenone, $5.8 \mathrm{mmol}$ of ethyl cyanoacetate and $5.8 \mathrm{mmol}$ ammonium acetate in propanol and refluxed at $110^{\circ} \mathrm{C}$ until 2-hydroxy-1-naphthaldehyde spot on TLC was judged to either be gone or reaction had ceased to progress further. Product was insoluble in propanol and precipitated out. Product was filtered, rinsed and then dried. MS (ESI) $m / z 355(\mathrm{M}+\mathrm{H})_{+} ; \mathrm{C}_{22} \mathrm{H}_{14} \mathrm{~N}_{2} 0_{3} ;{ }^{1} \mathrm{H}$ NMR (DMSO- $\left.d_{6}\right) \delta 7.01(\mathrm{~m}, 3 \mathrm{H}), 7.4(\mathrm{~m}, 1 \mathrm{H}), 7.58(\mathrm{~d}$, $\mathrm{J}=8.8 \mathrm{~Hz}, 1 \mathrm{H}), 7.67(\mathrm{t}, \mathrm{J}=7.2 \mathrm{~Hz}, 1 \mathrm{H}), 7.84(\mathrm{~m}, 1 \mathrm{H}), 8.02(\mathrm{~d}, \mathrm{~J}=7.2 \mathrm{~Hz}, 1 \mathrm{H}), 8.14(\mathrm{~d}, \mathrm{~J}=$ $8 \mathrm{~Hz}, 2 \mathrm{H}), 8.25$ (m, 3H), 13.44* (s, 1H), Yield 24\%.

*Peak indicates pyridine tautomer.

\section{3) 6-(2-hydroxyphenyl)-4-(3-nitrophenyl)-2-oxo-1,2-dihydropyridine-3- carbonitrile (Pyr4)}

$6.6 \mathrm{mmol}$ of 3-nitrobenzaldehyde was reacted with $6.6 \mathrm{mmol} 2^{\prime}$ -

hydroxyacetophenone, $6.6 \mathrm{mmol}$ of ethyl cyanoacetate and $6.6 \mathrm{mmol}$ ammonium acetate in propanol and refluxed at $110^{\circ} \mathrm{C}$ until 3-nitrobenzaldehyde spot on TLC was judged to either be gone or reaction had ceased to progress further. Propanol was evaporated and residue was washed with sodium bicarbonate. Product precipitated out of the aqueous solution was dried and washed with ethyl acetate and then recrystallized in acetonitrile at $-20^{\circ} \mathrm{C}$. MS (ESI) $m / z \quad 331.8(\mathrm{M}-\mathrm{H})-; \mathrm{C}_{18} \mathrm{H}_{11} \mathrm{~N}_{3} 0_{4} ;{ }^{1} \mathrm{H}$ NMR (DMSO-d $) \delta 6.65(\mathrm{~m}, 1 \mathrm{H}$ ), 
$6.67(\mathrm{~m}, 1 \mathrm{H}), 6.85(\mathrm{~s}, 1 \mathrm{H}), 7.1 \mathrm{ff} 8(\mathrm{~m}, 1 \mathrm{H}), 7.81(\mathrm{t}, \mathrm{J}=8 \mathrm{~Hz}, 1 \mathrm{H}), 7.87(\mathrm{~m}, 1 \mathrm{H}), 8.07(\mathrm{~m}$, $1 \mathrm{H}), 8.32(\mathrm{~m}, 1 \mathrm{H}), 8.40(\mathrm{~m}, 1 \mathrm{H})$, Yield $80 \%$.

\section{4) 6-(2-hydroxyphenyl)-2-oxo-4-(1H-pyrrol-2-yl)-1,2-dihydropyridine-3- carbonitrile (Pyr6)}

$1 \mathrm{mmol}$ of $1 \mathrm{H}$-pyrrole-2-carbaldehyde was reacted with $1 \mathrm{mmol} 2^{\prime}$ -

hydroxyacetophenone, $1 \mathrm{mmol}$ of ethyl cyanoacetate and $1 \mathrm{mmol}$ ammonium acetate in propanol and refluxed at $110^{\circ} \mathrm{C}$ until $1 \mathrm{H}$-pyrrole-2-carbaldehyde spot on TLC was judged to either be gone or reaction had ceased to progress further. Propanol was evaporated and residue was washed with sodium bicarbonate and ethylacetate. Organic phase was dried with magnesium sulfate and recrystallized at $-20^{\circ} \mathrm{C}$. MS (ESI) $m / z \quad 278(\mathrm{M}+\mathrm{H})_{+}$, $\mathrm{C}_{16} \mathrm{H}_{11} \mathrm{~N}_{3} \mathrm{O}_{2} ;{ }^{1} \mathrm{H}$ NMR (DMSO- $\left.d_{6}\right) \delta 6.47$ (m, 3H), 7.43 (overlapping m, 4H), 8.1 (slightly broad s, $3 \mathrm{H}-\mathrm{OH}) 8.5^{*}(\mathrm{~s}, 1 \mathrm{H}), \mathrm{N}-\mathrm{H}$ not visualized. Yield $<1 \%$

*Likely also in pyridine tautomer due to ppm shift being so far downfield.

\section{Results}

The nucleoside analogue synthesis procedure called for a 1:2.5:2 molar ratios for the 6-chloropurine riboside to the tetrahydroisoquinoline derivative and calcium carbonate in ethanol and refluxed at $85^{\circ} \mathrm{C}$ for 15 hours. Due to limited amounts of the tetrahydroisoquinoline starting reagents, they were made the limiting reactant. However, it was later determined that this resulted in a very difficult separation and the ratios were eventually returned to the original procedure.

Even with the tetrahydroisoquinoline derivatives as the excess reactant, in general the reaction resulted in poor yields under the proscribed conditions as $\mathrm{CaCO}_{3}$ was nearly insoluble. In addition, many of the tetrahydroisoquinoline starting materials were $\mathrm{HCl}$ salts, requiring far more base to produce the nucleophilic free base of the tetrahydroisoquinolines. $\mathrm{K}_{2} \mathrm{CO}_{3}$ was substituted for its improved solubility, and a catalytic amount of 18 -crown-6 was found effective at solvating the potassium ion. ${ }^{83}$ Depending on the electron donating or electron-withdrawing nature of the substitution on the tetrahydroisoquinolines and related reagents, the reactions often sometimes took longer 24 hours and rarely went to completion. However, poor solubility likely contributed the most to decreased yields. Some attempted reactions did not proceed, likely due to steric interactions. The same reactions were attempted in the microwave, but the ribose ring was very sensitive and tended to decompose, producing little to no product.

Table 4-1 shows the biological activity of the synthesized compounds. In addition to the parent compound known in these studies as compound-4 (Figure 4-4), ten compounds were successfully made, seven of which were tested for biological activity (Figure 4-1). The project was halted before testing was conducted on the other compounds (Figure 4-3). 
Table 4-1. Synthesized nucleoside derivative screening results

\begin{tabular}{lc}
\hline Compound & hENT1 IC $_{\mathbf{5 0}}$ (nM) \\
\hline HCPN01 & 40.98 \\
HCPN05 & 85.04 \\
HCPN09 & 53.77 \\
HCPN22 & Inactive \\
HCPN29 & $>1$ uM \\
HCPN30 & $>1 \mathrm{uM}$ \\
HCPN34 & 58.91 \\
\hline
\end{tabular}

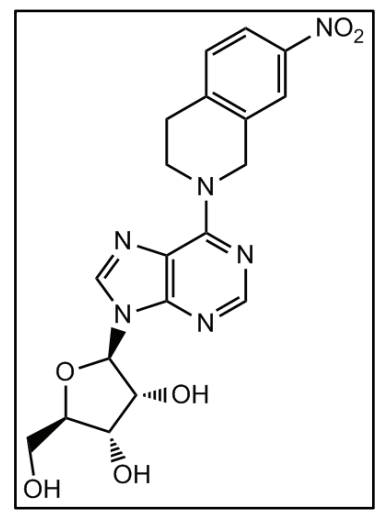

Figure 4-4. Compound-4 structure

Note: Reported hENT1 $\mathrm{IC}_{50} 0.45 \mathrm{nM}^{80}$ 
Exchanging the tetrahydroisoquinoline benzene ring system for heteroaromatic systems abolished activity, as did saturating the ring systems. HCPN01 and HCP09 which showed the best activity against hENT1, both have halogens in place of the nitro group found in the parent compound. Although they retained activity, they were far less potent than the parent compound. As halogens can undergo dipole interactions, this could explain the retention of some activity, but clearly it was not optimal. As nothing else in the molecule changed, this demonstrated the importance of the 7-position nitro group for potency and the sharp line between moderate activity and potent inhibitor. This agreed with prior studies which showed that analogues showed decreased activity when the nitro groups was moved to other positions on the tetrahydroisoquinoline ring. Further, the nitrile group, which instead occupies the five-position may be able to partially interact with the transporter, but with decreased affinity. A compound with the nitrile in the same position would potentially retain activity as the nitrile group could participate in an electrostatic interaction, depending on the steric properties of the binding pocket. As such, synthesizing a molecule of that nature could further elucidate the interactions of inhibitor and transporter at that position.

Figure 4-3 shows the synthesized pyridone analogues. As both lead compounds contained hydrogen bond acceptors in the four substituted position, it was hypothesized that it was required for activity. Indeed, compounds without a hydrogen bond acceptor had no activity (Figure 4-6). The pyrrole ring in Pyr 6 would be protonated at physiological $\mathrm{pH}$ and under assay conditions, and as such would not be a hydrogen bond acceptor, but rather a donor. Unfortunately none of the analogues showed as significant activity as the parent compounds and more would have to be synthesized to expand the structure activity relationship. 


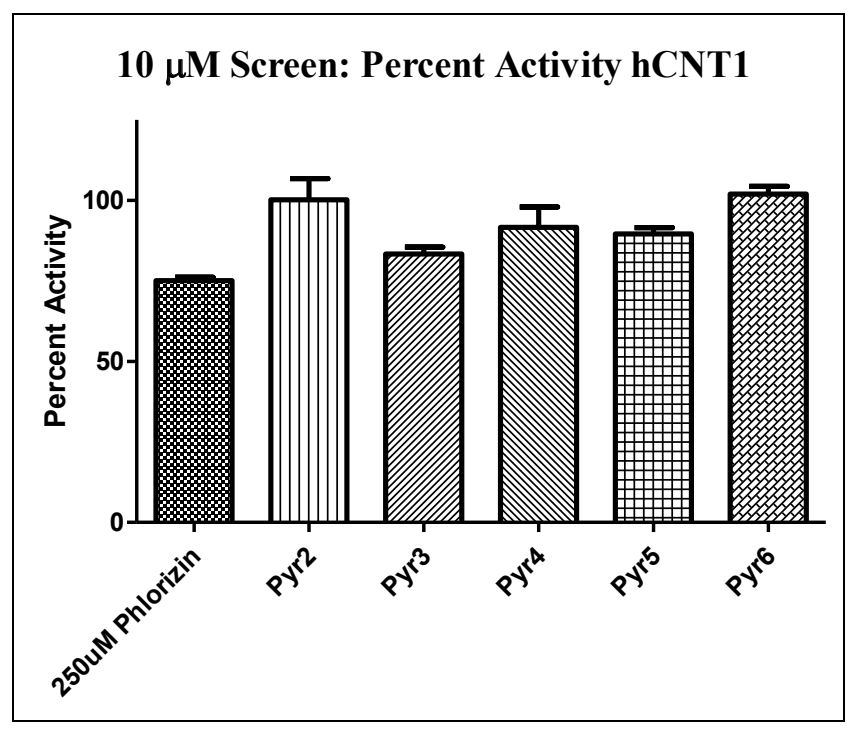

Figure 4-5. Pyridone screening results 


\section{CHAPTER 5. BROAD INSTITUTE COLLABRATIVE STUDY}

\section{Introduction}

Dr. Timothy Lewis and his group at the broad institute at Harvard synthesized a series of dilazep analogues to explore the structure-activity relationship in regards to ENT1 and ENT2 (submitted for publication). Prior work had identified hENT1 inhibitors dipyridamole and dilazep as novel potential agents for use in improving the efficacy of oncolytic herpes simplex virus-1 replication rate. ${ }^{52}$ In contrast to many chemotherapies currently used to treat cancer, oHSV1 only targets cancerous cells. Unfortunately oHSV1 use in the clinic is limited by poor replication rate and spread to nearby cancerous cells. The discovery of the potential synergistic role of ENT1 and ENT2 inhibitors could increase the utility of this novel cancer treatment method.

\section{Methods}

The compounds were screened in duplicate in the same manner as described in Chapter 3. Dose response assays of hits were conducted in triplicates and non-linear regression curves were calculated with least squares method in GraphPad Prism 5.

Compounds that demonstrated significant and selective inhibition were explored further with dose dependent assays.

\section{Results}

Figures 5-1 through 5-3 show the biological screening data while Table 5-1 lists the $\mathrm{IC}_{50} \mathrm{~S}$ of the biologically potent compounds. With the exception of $\mathrm{M} 7$, all the tested compounds showed similar screening results when tested against hENT2, although the trend showed the rENT2 perhaps proving slightly more sensitive to the more potent compounds (Figure 5-4). M7 had poor activity against both, so its deviation is not of significant importance.

While dilazep (Figure 5-5) inhibited hENT1 and (r/h)ENT2, ENT2 exhibited over five hundred-fold less sensitivity. Although no potent ENT2 selective compound was identified, cell screening identified four compounds with increased potency against ENT2 and similar or increased potency against hENT1.

Dilazep (Figure 5-5) and close analogues (Figure 5-6) proved to be potent hENT1 inhibitors $\left(\mathrm{IC}_{50}<100 \mathrm{nM}\right.$ ) with little or no activity against rENT2. Modification of the homopiperazine was tolerated with replacement using a piperazine, or methylsubstitued piperazine ring. In addition the alkyl chains showed tolerance to length alteration with both one carbon longer and shorter retaining potency. Replacement of 


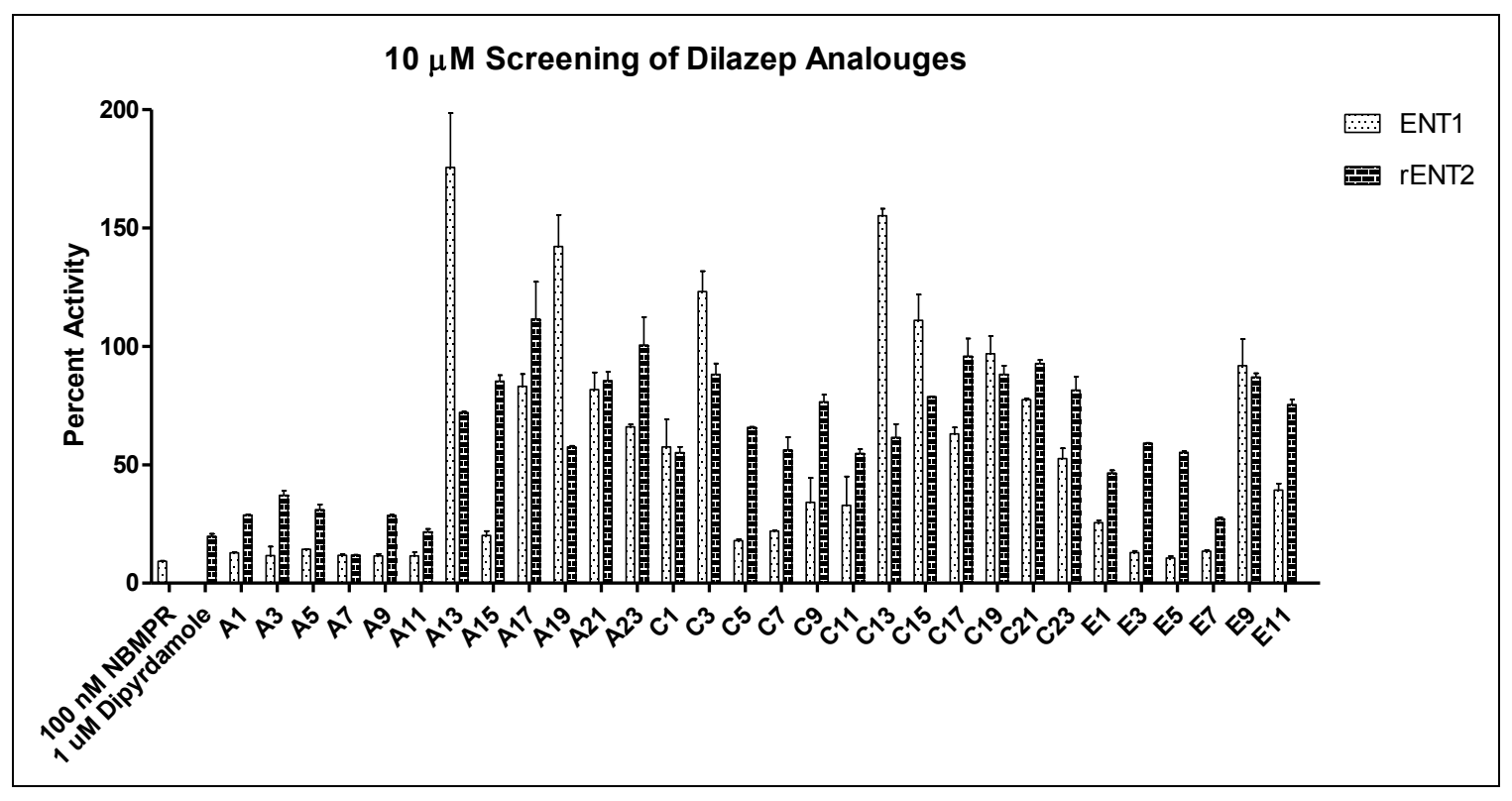

Figure 5-1. $\quad 10 \mu \mathrm{M}$ screening of A1-E11 against hENT1 and rENT2

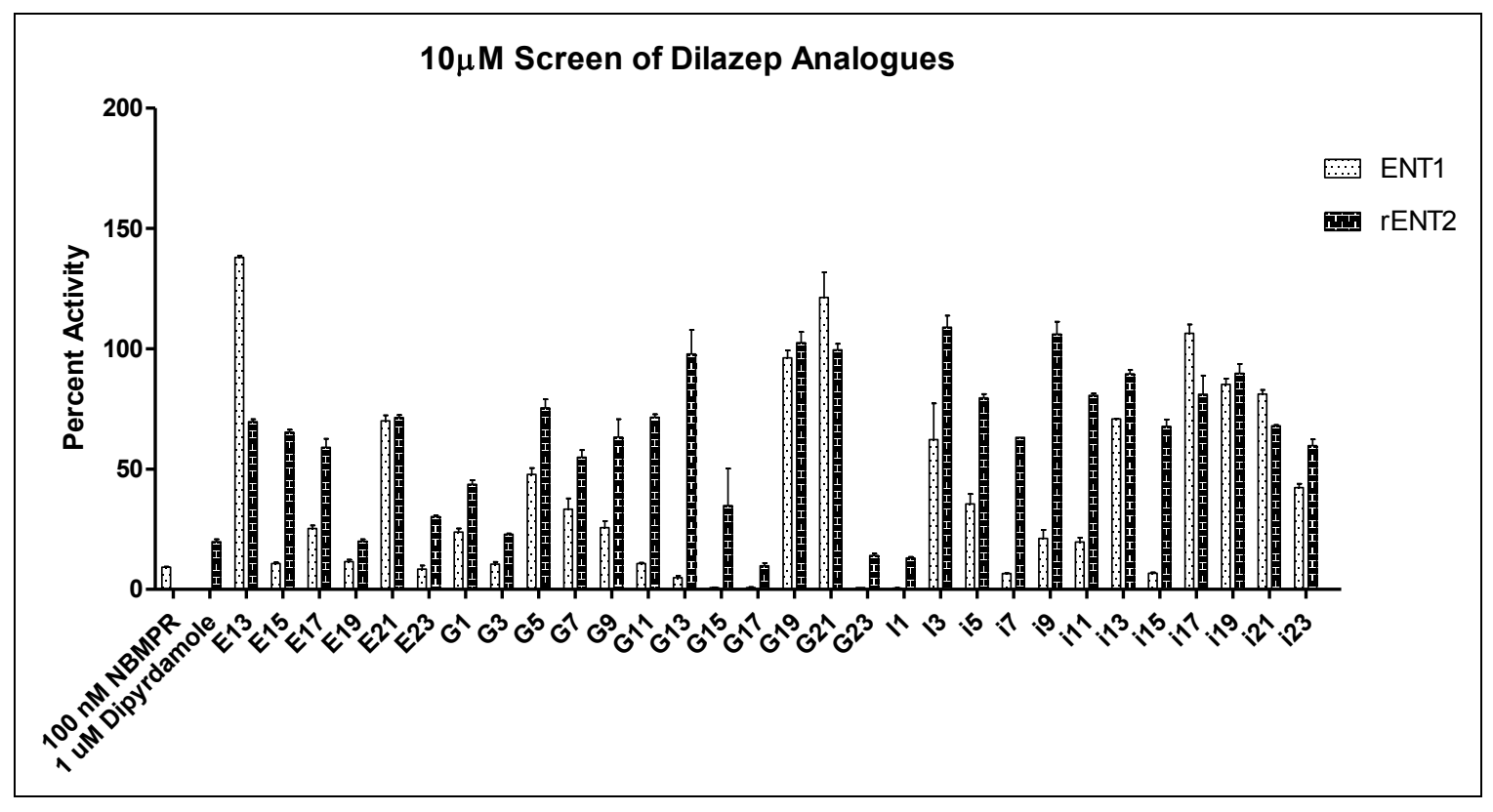

Figure 5-2. $\quad 10 \mu M$ screening of E13-I23 against hENT1 and rENT2 


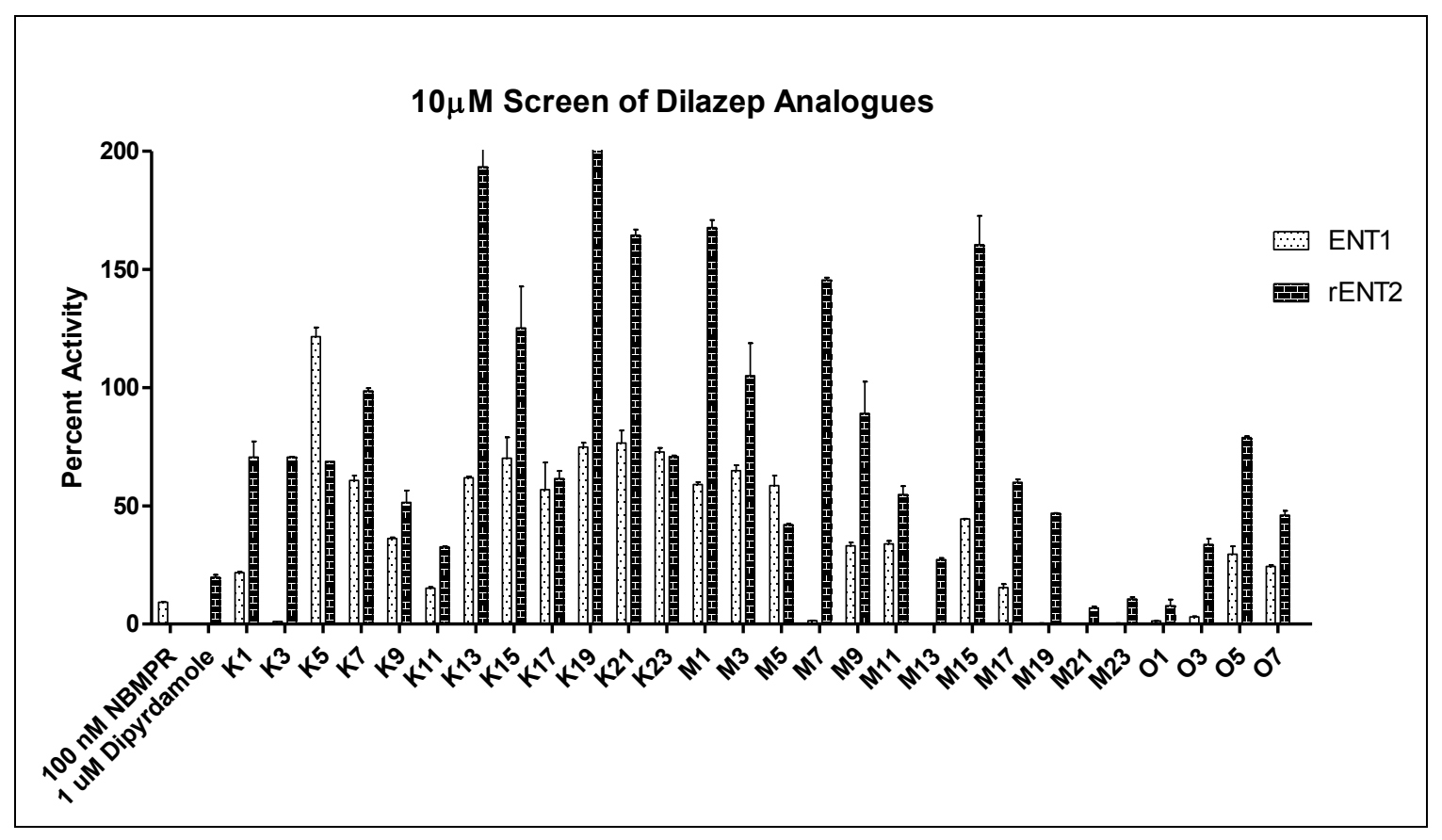

Figure 5-3. $\quad 10 \mu M$ screening of K1-O7 against hENT1 and rENT2 
Table 5-1. Dilazep analogue $\mathrm{IC}_{50} \mathrm{~s}$

\begin{tabular}{|c|c|c|}
\hline Compound & $\begin{array}{c}\text { hENT1 IC } \text { IC }_{\mathbf{5 0}}[\mathbf{n M}] \\
(95 \% \mathrm{CI})\end{array}$ & $\underset{(95 \% \mathrm{CI})}{\text { rENT2 }_{\text {IC }}[\mathbf{n M}]}$ \\
\hline I5(M21) & $\begin{array}{c}31.0 \\
(13.4-71.7)\end{array}$ & $* * *$ \\
\hline G23 ${ }^{\text {Dilazep }}$ & $\begin{array}{l}17.5 \\
(9.4-33.8)\end{array}$ & $\begin{array}{c}8795 \\
(2075-37280)\end{array}$ \\
\hline 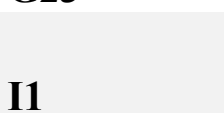 & $\begin{array}{c}66.1 \\
(35.7-122)\end{array}$ & $\begin{array}{c}1309 \\
(897.7-1909)\end{array}$ \\
\hline $\mathbf{I 5}$ & $\begin{array}{c}93.9 \\
(27.9-315.7)\end{array}$ & $* * *$ \\
\hline $\mathbf{I 7}$ & $\begin{array}{c}802.6 \\
(503.4-1280)\end{array}$ & $* * *$ \\
\hline I9 & $\begin{array}{c}7136 \\
(1564-32.6 \mu \mathrm{M})\end{array}$ & $* * *$ \\
\hline $\mathbf{K 3}$ & $\begin{array}{c}216.9 \\
(112.4-418.6)\end{array}$ & $* * *$ \\
\hline M13 & $\begin{array}{l}2.8 \\
(1.6-4.7)\end{array}$ & $\begin{array}{c}977 \\
(598-1690)\end{array}$ \\
\hline M19 & $\begin{array}{l}4.6 \\
(2.5-8.6)\end{array}$ & $* * *$ \\
\hline M23 & $\begin{array}{c}17.7 \\
(12.8-25.7)\end{array}$ & $\begin{array}{c}1074 \\
(642.5-1796)\end{array}$ \\
\hline 01 & $\begin{array}{l}3.2 \\
(1.7-6.2)\end{array}$ & $\begin{array}{c}1488 \\
(843.2-2625)\end{array}$ \\
\hline
\end{tabular}




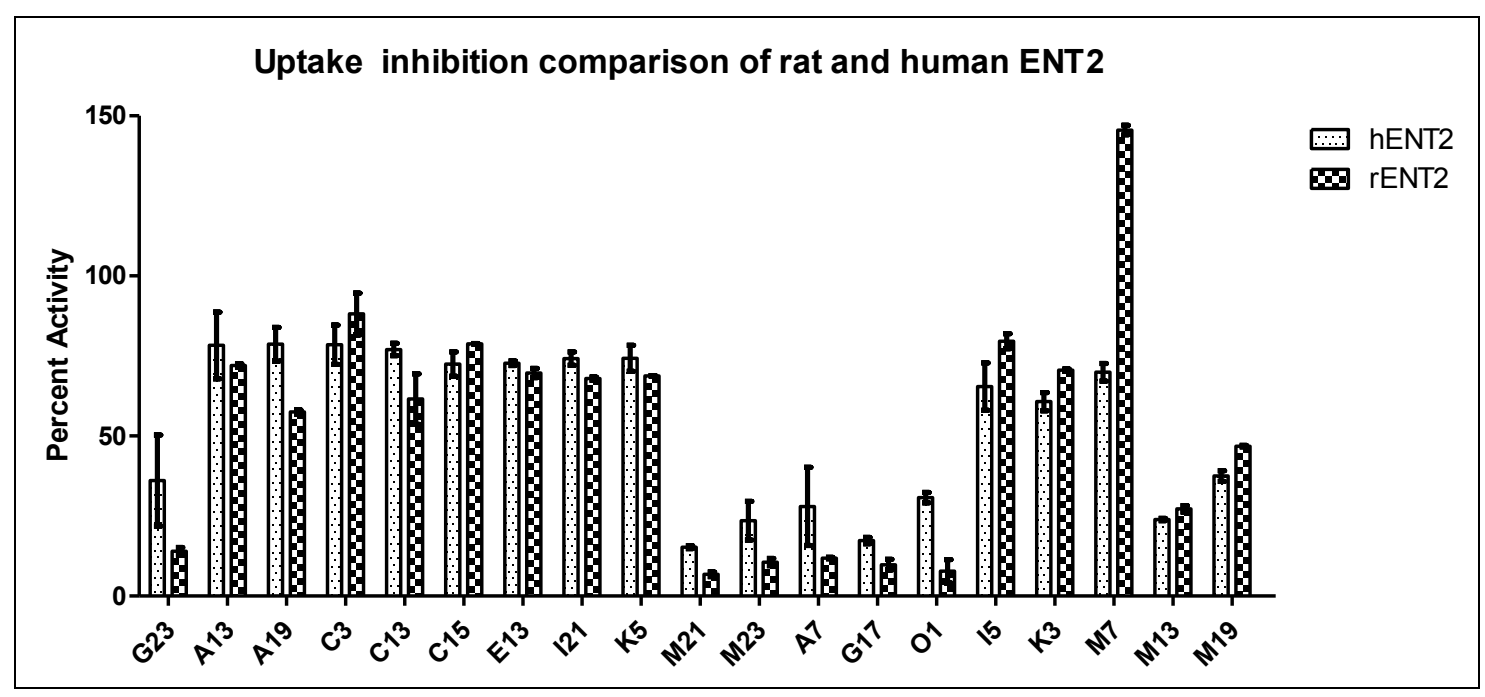

Figure 5-4. Uptake inhibition comparison of rat and human ENT2 


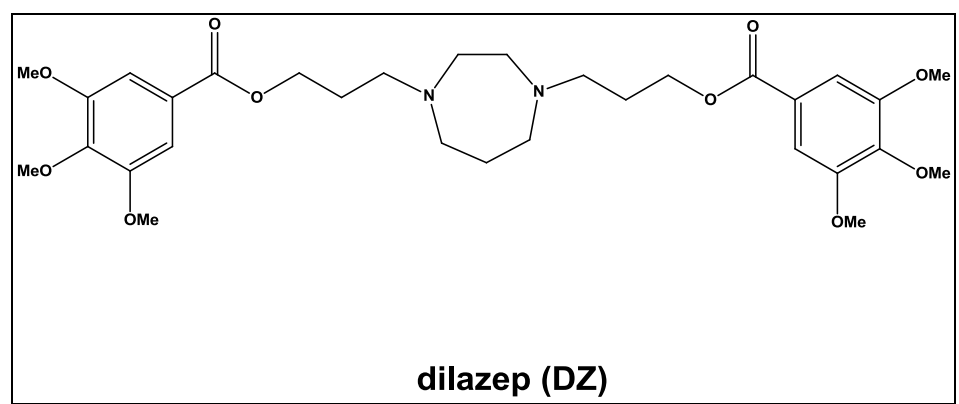

Figure 5-5. Dilazep structure

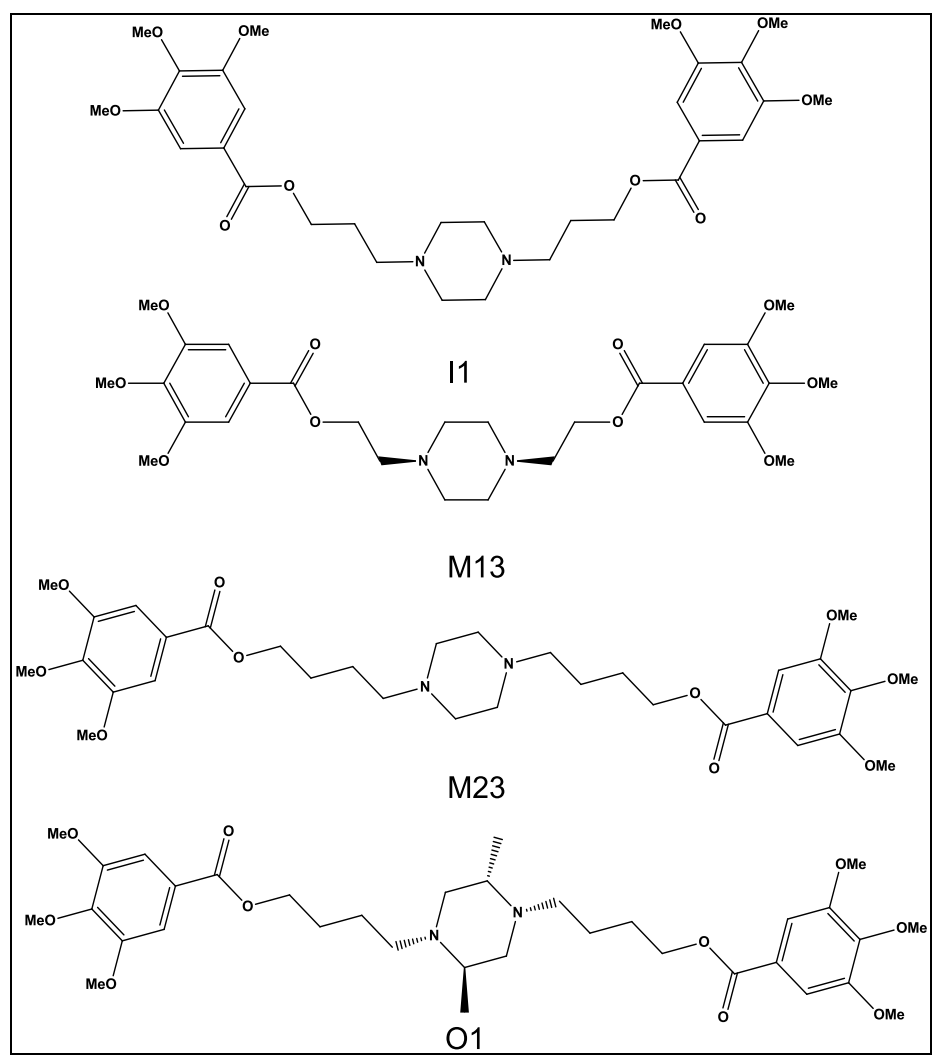

Figure 5-6. Potent dilazep analogues 
ester bonds with amides retained activity, but replacement with ether or heterocycle greatly diminished hENT1 activity was diminished. The 3,4,5-(OMe $)_{3}$ substituted phenyl rings showed the best activity, but two of the groups are not required based on the activities of I7 and K3, in which only one methoxy is required for hENT1 activity. While one compound M13 demonstrated ENT2 activity just under $1 \mu \mathrm{M}$, no potent, selective ENT2 inhibitor was identified (Table 5-1). Based on the results, it was concluded that the mechanism of improvement of oHSV1 replication efficiency when treated with dilazep involved ENT1 rather than ENT2. More mechanistic studies are required to elucidate the exact mechanism. The data have been submitted for publication. 


\section{CHAPTER 6. CONCLUSION AND FUTURE STUDIES}

The collective results of this work highlight the difficulties faced in drug discovery when the structure of the target is not known. While fourteen hENT1 hits and three hENT4 hits were found serendipitously with the hCNT3 pharmacophore, the initial screen failed at finding any hCNT3 hits. Later, when the biological data used to build the pharmacophore were reviewed, it was determined that some of the compounds were potentially substrates or rapid reversible inhibitors, but as previously described, further kinetic studies would be required to ascertain which. To confirm substrates, one method might involve tagging the test compound with a radio isotope and determining if it is taken into the cell. Several initial hits demonstrated the phenomenon of appearing potent, but upon retesting with a different incubation period, showed marked decrease or total lack of apparent inhibition. This highlights that with regards to molecular modeling, pinpointing that the key features between substrate and inhibitor are not particularly well defined. The failure of the screen shows the most significant limitation to molecular modeling with regards to 3D-QSAR methods in that models are only as good as the biological data used for analysis.

Looking at the well characterized hENT1, with many known compounds that can inhibit it at a wide variety of concentrations, it is interesting to note the differences in the flavonoid models versus the NBMPR models or even the dipyridamole models. The highly potent dipyridamole and NMBPR analogues both yielded statistically significant, predictive models when aligned with a seven-point pharmacophore. However, the moderate potency flavonoids only produce a five-point pharmacophore. Taken with the ability of the dipyridamole model using four pharmacophore points to identify the UC compounds found to be biologically active, one could conjecture that a minimum of four pharmacophore points are required for activity, but not necessarily the same four points. The synthesized molecules, which were highly similar to the potent Compound-4 showed a marked decrease in activity, demonstrating that very small changes can sharply increase or decrease the potency of a potential inhibitor, and that while the transporters have a broad spectrum of compounds they transport, it is possible to optimize a compound to selectively and potently target a specific transporter. Even at their current potency, several of the synthesized compounds are well within pharmacologically relevant ranges. They could be tested against bacteria or for toxicity in cancer cell lines, as antimetabolites have been a proven efficacious route.

While the other nucleoside transporters lack the compound diversity for a similar molecular modeling comparison, based on their functionality and over-lapping permeant selectivity one might hypothesis that for each transporter there is a nucleoside-based pharmacophore and at least one or more potential pharmacophores for non-nucleoside molecules. Further work to expand series of compounds for each of the transporters could serve to test that postulate.

The hCNT1 screening proved to be most productive, especially given the limited size of the original data set. Likewise, the Vibrio cholera crystal structure allowed for the generation of a homology model that also successfully identified active compounds, 
albeit only one, HM50, of more than moderate micromolar potency. While screening of synthesized pyridones yielded little in the way of conclusive results, the synthesized molecules were few in number, and a much larger library is needed to expand the structure-activity relationship, especially in regards to differentiating substrate versus inhibitor pharmacophore points. While the original model indicated hydrogen bond donor and aromatic rings as important pharmacophore elements, the hit-enriched model suggested that hydrogen bond acceptors played a more important role, which the trend in the biological data seemed to support, but expansion of the data set is required to fully test this theory. This is suggested as the focus of future work.

In regards to the collaborative work, it resulted in some very promising compounds of nanomolar potency, even though the goal of producing an ENT2 selective compound was not met. The identified compounds could potentially lead to an adjunct therapy for improving the efficacy of oHSV1 treatments. The blockade of the nucleoside transporters would prevent the cells from taking up nucleosides to replicate and allow the intercellular nucleosides to be used by oHSV1. This could potentially cause cell arrest, giving oHSV1 time to replicate enough to spread to nearby cancerous cells.

Of particularly interesting note, when looking at dilazep and comparing it to the most potent hENT1 inhibitor found in the initial UC compound screening the (UC21), one can see a similarity in structure. UC21 possesses fewer flexible bonds, but retains the tri-methoxy substitution pattern (Figure 6-1). Also similar to the dilazep analogues, UC21 concurs with the trend that a symmetrical molecule is not required for activity. Both the NBMPR and dipyridamole pharmacophores suggest that symmetry is not required, which provides another potential area of molecular modifications.

It is possible that in some cases such as with hENT1 and hENT2, due to the similarity of the transporters, compounds with only minor changes to the same core structure may elucidate SAR for a series but fail to achieve selectivity as specific cores in and of themselves may be more selective toward a specific transporter. As mentioned before, hENT4 shares less similarity and utilizes alternative methods of functionality compared to hENT1 and hENT2. As such, it is interesting that selective and potent dipyridamole analogues were identified for both $\mathrm{hENT} 1{ }^{70}$ and hENT4. ${ }^{1}$ Then again, dipyridamole is known to interact with other molecules and its nucleoside transporter activity was discovered after the fact. Dipyridamole is a phosphodiesterase 3 (PDE3) inhibitor, demonstrating that often compounds may achieve the desired biological response through interacting with more than one target in vivo. ${ }^{84}$

While the drug potentials are still far off on the horizon, exploring the roles of nucleoside transporters is crucial to understanding cellular signaling, transport, and the role they play both in normal cells and in diseased states. This study identified a number of compounds for which derivations can be used for that purpose. In addition, the hENT1 models, in particular the dipyridamole pharmacophore model and the flavonoid hCNT1 pharmacophore model can aid in identifying potential active compounds. 


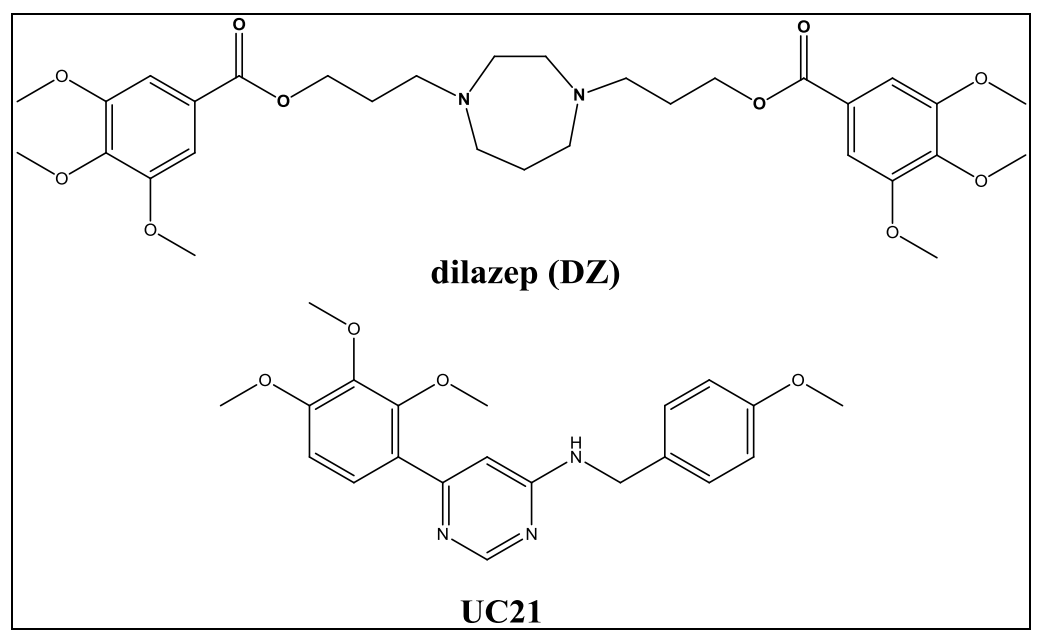

Figure 6-1. Dilazep and UC21 comparison 


\section{LIST OF REFERENCES}

1. Chunmei Wang; Lin, W.; Playa, H.; Sun, S.; Cameron, K.; Buolamwini, J. K. $J$ Biol Chem 2013, 86, 1531.

2. $\quad$ Young, J. D.; Yao, S. Y. M.; Baldwin, J. M.; Cass, C. E.; Baldwin, S. A. Molecular Aspects of Medicine 2013, 34, 529.

3. Pastor-Anglada, M.; Cano-Soldado, P.; Errasti-Murugarren, E.; Casado, F. J. Xenobiotica 2008, 38, 972.

4. Buolamwini, J. K. Current Medicinal Chemistry 1997, 4, 31.

5. Vijayalakshmi, D.; Belt, J. A. J Biol Chem 1988, 263, 19419.

6. Young, J. D.; Yao, S. Y. M.; Sun, L.; Cass, C. E.; Baldwin, S. A. Xenobiotica 2008, 38, 995.

7. Baldwin, S. A.; Beal, P. R.; Yao, S. Y. M.; King, A. E.; Cass, C. E.; Young, J. D. Eur J Physiol 2004, 441, 8.

8. Smith, K. M.; Ng, A. M. L.; Yao, S. Y. M.; Labedz, K. A.; Knaus, E. E.; Wiebe, L. I.; Cass, C. E.; Baldwin, S. A.; Chen, X.-Z.; Karpinski, E.; Young, J. D. J Physiol 2004, 558, 17.

9. $\quad$ MW, R.; SY, Y.; AM, N.; JR, M.; CE, C.; JD, Y. Mol Membr Biol 1998, 15, 203.

10. Johnson, Z. L.; Cheong, C.-G.; Lee, S.-Y. Nature 2012, 483, 6.

11. Ritzel, M. W. L.; Ng, A. M. L.; Yao, S. Y. M.; Graham, K.; Loewen, S. K.; Smith, K. M.; Ritzeli, R. G.; Mowles, D. A.; Carpenter, P.; Chen, X.-Z.; Karpinski, E.; Hyde, R. J.; Baldwin, S. A.; Cass, C. E.; Young, J. D. J Biol Chem 2001, 276, 2914.

12. Errasti-Murugarren, E.; Molina-Arcas, M.; Casado, F. J.; Pastor-Anglada, M. The FASEB Journal 2009, 23, 172.

13. Yao, S. Y. M.; Ng, A. M. L.; Cass, C. E.; Baldwin, S. A.; Young, J. D. J Biol Chem 2011, 286, 32552.

14. Zhou, M.; Duan, H.; Engel, K.; Xia, L.; Wang, J. Drug Metabolism and Disposition 2010.

15. Hagberg, H. Plugers Arch 1985, 404, 342.

16. Barnes, K.; Dobrzynski, H.; Foppolo, S.; Beal, P. R.; Ismat, F.; Scullion, E. R.; Sun, L.; Tellez, J.; Ritzel, M. W. L.; Claycomb, W. C.; Cass, C. E.; Young, J. D.; Billeter-Clark, R.; Boyett, M. R.; Baldwin, S. A. Circ Res 2006, 99, 510.

17. MW, R.; SY, Y.; MY, H.; JF, E.; CE, C.; JD, Y. Am J Physiol 1997, 272, C707.

18. Huang, Q.-Q.; M., S. Y.; YaoS; Ritzel, M. W. L.; Paterson, A. R. P.; Cass, C. E.; JD, Y. J Biol Chem 1994, 269, 17757.

19. Hamilton, S. R.; Yao, S. Y. M.; Ingram, J. C.; Hadden, D. A.; Ritzel, M. W. L.; Gallagheri, M. P.; Henderson, P. J. F.; Cass, C. E.; Young, J. D.; Baldwin, S. A. J Biol Chem 2001, 276, 27981.

20. Slugoski, M. D.; Ng, A. M.; Yao, S. Y.; Lin, C. C.; Mulinta, R.; Cass, C. E.; Baldwin, S. A.; Young, J. D. J Biol Chem 2009, 284, 17281.

21. Morgan, N. V.; Morris, M. R.; Cangul, H.; Gleeson, D.; Straatman-Iwanowska, A.; Davies, N.; Keenan, S.; Pasha, S.; Rahman, F.; Gentle, D.; Vreeswijk, M. P. G.; al, e. PLoS Genetics 2010, 6, 9. 
22. Knight, D.; Harvey, P. J.; Iliadi, K. G.; Klose, M. K.; Iliadi, N.; Dolezelova, E.; Charlton, M. P.; Zurovec, M.; Boulianne, G. L. The Journal of neuroscience : the official journal of the Society for Neuroscience 2010, 30, 5047.

23. Choi, D. S.; Cascini, M. G.; Mailliard, W.; Young, H.; Paredes, P.; McMahon, T.; Diamond, I.; Bonci, A.; Messing, R. O. Nature neuroscience 2004, 7, 855.

24. Furihata, T.; Fukuchi, Y.; Iikura, M.; Hashizume, M.; Miyajima, A.; Nagai, M.; Chiba, K. Antimicrobial agents and chemotherapy 2010, 54, 3035.

25. Cass, C. E.; Gaudette, L. A.; Paterson, A. R. Biochim Biophys Acta 1974, 345, 1.

26. Elion, G. B. Fed Proc 1967, 26, 898.

27. Benedict, W. F.; Baker, M. S.; Haroun, L.; Choi, E.; Ames, B. N. Cancer Res 1977, 37, 2209.

28. Waidmann, O.; Pleli, T.; Dvorak, K.; Baehr, C.; Mondorf, U.; Plotz, G.; Biondi, R. M.; Zeuzem, S.; Piiper, A. J Biol Chem 2009, 284, 32256.

29. JL, W.; A, S.; ZP, M.; CM, T. J Biol Chem 2000, 275, 6.

30. Hammond, J. R. Naunyn Schmiedebergs Arch Pharmacol 2000, 361, 373.

31. Lin, W., University of Tennessee2006.

32. Baldwin, S. A.; Yao, S. Y. M.; Hyde, R. J.; Ng, A. M. L.; Foppolo, S.; Barnes, K.; Ritzel, M. W. L.; Cass, C. E.; Young, J. D. J Biol Chem 2005, 280, 15880.

33. Tatani, K. N., Y.; Kikuchi, N.; 2006.

34. Gupte, A.; Buolamwini, J. K. Bioorg Med Chem Lett 2009, $19,917$.

35. L, R.; D, S.; GI, S.; D, P.; RA, D. J Clin Invest 1987, 79, 1510.

36. Toan, S. V.; To, K. K.; Leung, G. P.; de Souza, M. O.; Ward, J. L.; Tse, C. M. Pflugers Archiv : European journal of physiology 2003, 447, 195.

37. Zimmerman, E.; Huang, M.; Leisewitz, A.; Wang, Y.; Yang, J.; Graves, L. M. FEBS Lett. 2009, 583, 425.

38. Gray, J. H.; Mangravite, L. M.; Owen, R. P.; Urban, T. J.; Chan, W.; Carlson, E. J.; Huang, C. C.; Kawamoto, M.; Johns, S. J.; Stryke, D.; Ferrin, T. E.; Giacomini, K. M. Molecular Pharmacology 2004, 65, 512.

39. Owen, R. P.; Badagnani, I.; Giacomini, K. M. J Biol Chem 2006, 281, 26675.

40. Badagnani, I.; Chan, W.; Castro, R.; Brett, C.; Huang, C.; Stryke, D.; Kawamoto, M.; Johns, S.; Ferrin, T.; Carlson, E.; Burchard, E.; Giacomini, K. The

Pharmacogenomics Journal 2005, 5, 157.

41. Huang, M.; Wang, Y.; Collins, M.; Gu, J. J.; Mitchell, B. S.; Graves, L. M. J Biol Chem 2002, 277, 28364.

42. Visser, F.; Vickers, M. F.; Ng, A. M. L.; Baldwin, S. A.; Young, J. D.; Cass, C. E. J Biol Chem 2002, 277, 395.

43. Leung, G.; Man, R.; Tse, C. Biochemical Pharmacology 2005, 70, 355.

44. T, Y.; Y, M.; Y, F.; S, T.; Z, T.; T, T.; K, H.; T, H. Pharmacology 2000, 60, 34.

45. Striessnig, J.; Zernig, G.; Glossman, H. Eur J Pharmacol. 1985, 150, 67.

46. $\quad$ NOTT, M. W. Br. J. Pharmac. 1970, 39, 287.

47. Damaraju, V. L.; Scriver, T.; Mowles, D.; Kuzma, M.; Ryan, A. J.; Cass, C. E.; Sawyer, M. B. Clinical Cancer Research 2014, 20, 176.

48. Zhang, J.; Visser, F.; King, K. M.; Baldwin, S. A.; Young, J. D.; Cass, C. E. Cancer Metastasis Rev 2007, 26, 25.

49. Govindarajan, R.; Leung, G. P. H.; Zhou, M.; Tse, C.-M.; Wang, J.; Unadkat, J. D. Am J Physiol Gastrointest Liver Physiol 2009, 296, 14. 
50. Stephen W. Ely, P., MD, ; Robert M. Berne, M. Circulation 1992, 85, 13.

51. Grover, G. J.; Sleph, P. G. Journal of Pharmacology and Experimental Therapeutics 1993, 268, 90.

52. Passer, B. J.; Cheema, T.; Zhou, B.; Wakimoto, H.; Zaupa, C.; Razmjoo, M.; Sarte, J.; Wu, S.; Wu, C. 1.; Noah, J. W.; Li, Q.; Buolamwini, J. K.; Yen, Y.;

Rabkin, S. D.; Martuza, R. L. Cancer Research 2010, 70, 3890.

53. Junger, W. G. Nat Rev Immunol 2011, 11, 201.

54. Ye, J.-H. World Journal of Gastroenterology 2009, 15, 4491.

55. Parker, M., D; Hyde, R. J.; Yao, S. Y. M.; McRobert, L.; Cass, C. E.; Young, J. D.; McConkey, G. A.; Baldwin, S. A. Biochemical Journal 2000, 349, 8.

56. Gupte, A.; Buolamwini, J.; Yadav, V.; Chu, C.; Naguib, F.; Elkouni, M.

Biochemical Pharmacology 2005, 71, 69.

57. Cramer, R. D. I.; Patterson, D. E.; Bunce, J. D. Journal of the American Chemical Society 1988, 110, 8.

58. Klebe, G.; Abraham, U.; Mietzner, T. Journal of Medicinal Chemistry 1994, 37, 16.

59. Kier, L. Mol. Pharmacol. 1967, 3, 487.

60. Lemmen, C.; Lengauer, T. J Comput Aided Mol Des 2000, 14, 215.

61. Klebe, G.; Abraham, U.; Mietzner, T. Journal of Medicinal Chemistry 1994, 37, 4130.

62. Jarvis, S. M.; Hall, C. A. Biochimica et Biophysica Acta 1992, 1105, 300.

63. Chang, C.; Swaan, P. W.; Ngo, L. Y.; Lum, P. Y.; Patil, S. D.; Unadkat, J. D. Mol. Pharmacol. 2004, 65, 558.

64. Zhu, Z.; Buolamwini, J. K. Bioorganic \& Medicinal Chemistry 2008, 16, 3848.

65. Gupte, A.; Buolamwini, J. K. Bioorganic \& Medicinal Chemistry Letters 2009, $19,314$.

66. Paterson, A. R.; Naik, S. R.; Cass, C. E. Mol Pharmacol 1977, 13, 1014.

67. M, S.-G.; R, P.; Paul B, P. Acta Cryst. 1984, C40, 1897.

68. Quail, J. W. Acta Cryst. 1988, C44, 1614.

69. Ho, H. T.; Pan, Y.; Cui, Z.; Duan, H.; Swaan, P. W.; Wang, J. J Pharmacol Exp Ther 2011, 339, 376.

70. Wang, C.; Pimple, S.; Buolamwini, J. K. Biochemical Pharmacology 2010, 79, 307.

71. Hu, H.; Endres, C. J.; Chang, C.; Umapathy, N. S.; Lee, E. W.; Fei, Y. J.; Itagaki, S.; Swaan, P. W.; Ganapathy, V.; Unadkat, J. D. Mol Pharmacol 2006, 69, 1542.

72. Schrödinger, LLC: New York, NY, 2012.

73. Schrödinger, LLC: New York, NY, 2013.

74. Tripos International: 1699 South Hanley Rd., St. Louis, Missouri, 63144, USA.

75. Sundaram, M.; Yao, S. Y.; Ng, A. M.; Griffiths, M.; Cass, C. E.; Baldwin, S. A.; Young, J. D. J Biol Chem 1998, 273, 21519.

76. Zhou, M.; Xia, L.; Engel, K.; Wang, J. J Biol Chem 2007, 282, 3188.

77. Kenakin, T. A Phamacology Primer; 30 Corporate Drive, Suite 400, Burlington MA, 01803, USA: Academic Press an imprint of Elsevier, 2010.

78. $\quad$ Kimes, B. W.; Brandt, B. L. Exp Cell Res 1976, 98, 367.

79. Wang, C.; Pimple, S.; Buolamwini, J. K. Biochem Pharmacol 2010, 79, 307. 
80. Zhu, Z.; Furr, J.; Buolamwini, J. K. Journal of Medicinal Chemistry 2003, 46, 831.

81. Yu, Y.; Hu, Y.; Shao, W.; Huang, J.; Zuo, Y.; Huo, Y.; An, L.; Du, J.; Bu, X. European Journal of Organic Chemistry 2011, $2011,4551$.

82. El-Sayed, H. A.; Ouf, N. H. Nature and Science 2012, 10, 59.

83. Rounaghi, G.; Eshaghi, Z.; Ghiamati, E. Talanta 1997, 44, 275.

84. Fukawa, K.; Saitoh, K.; Irino, O.; Ohkubo, K.; Hashimoto, S. Thrombosis research 1982, $27,333$. 


\section{Modeling Data}

\section{hENT1 Models}

\section{Flavonoid CoMFA Contours}

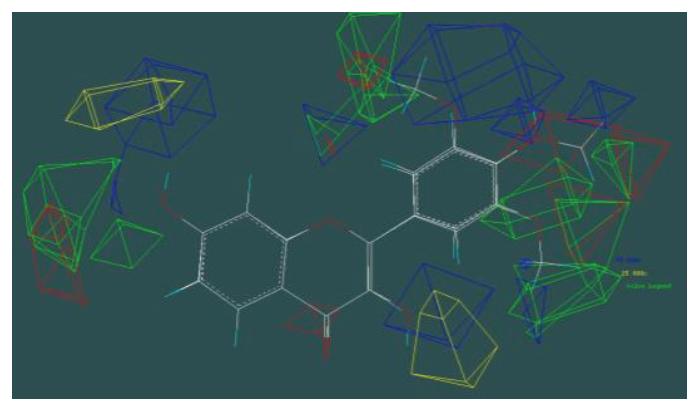

Figure A-1. Flavonoid CoMFA contours of hENT1

Note: CoMFA color key. Steric: green- favored, yellow- disfavored; electrostatics: bluefavored, red- disfavored.

\section{Dipyridamole CoMFA and CoMSIA Contours}

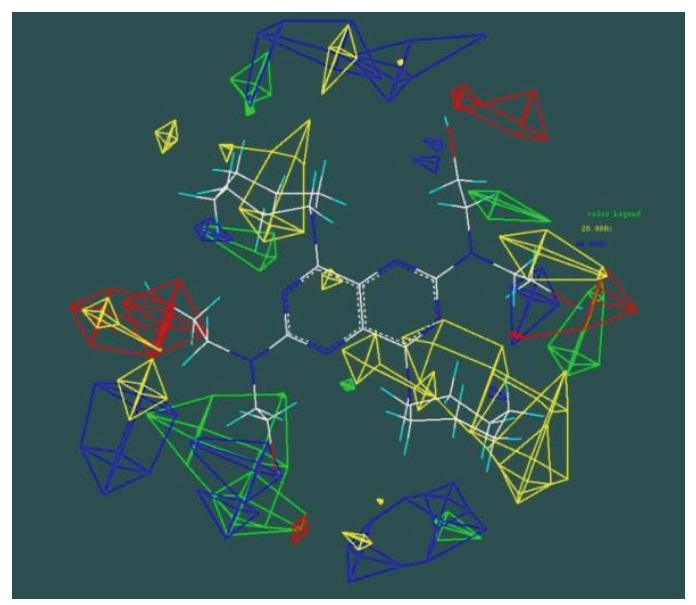

Figure A-2. hENT1 dipyridamole CoMFA contours

Note: CoMFA color key. Steric: green- favored, yellow- disfavored; electrostatics: bluefavored, red- disfavored. 


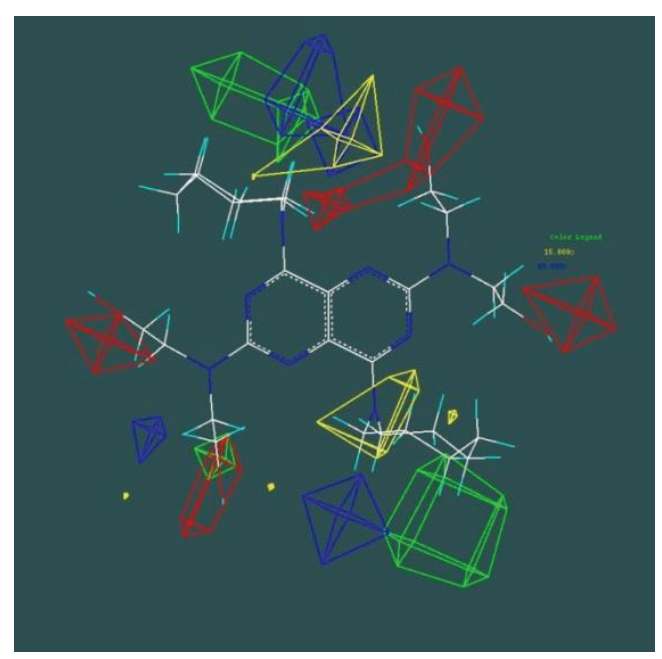

Figure A-3. hENT1 dipyridamole CoMSIA electrostatic and steric contours

Note: CoMSIA color key. Steric: green- favored, yellow- disfavored; electrostatic: bluefavored, red-disfavored

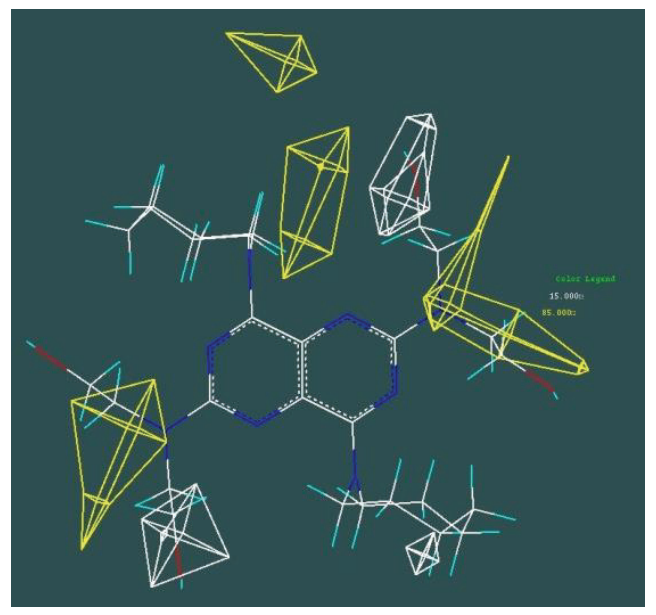

Figure A-4. hENT1 dipyridamole CoMSIA hydrophobic contours

Note: CoMSIA color key. Hydrophobic: yellow- favored, white- disfavored 


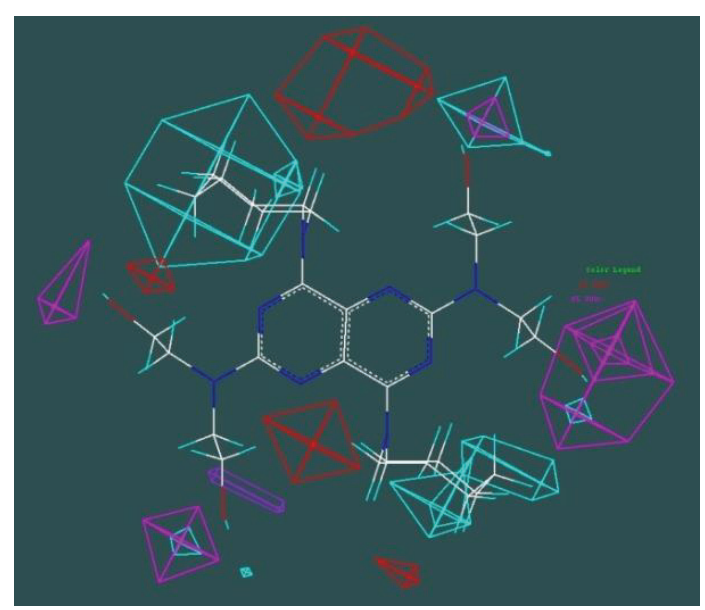

Figure A-5. hENT1 dipyridamole CoMSIA hydrogen bond donor and acceptor contours

Note: CoMSIA color key. Hydrogen-bond donor: cyan- favored, purple- disfavored; Hydrogen-bond acceptor: magenta- favored, red- disfavored.

\section{NBMPR CoMFA and CoMSIA Contours}

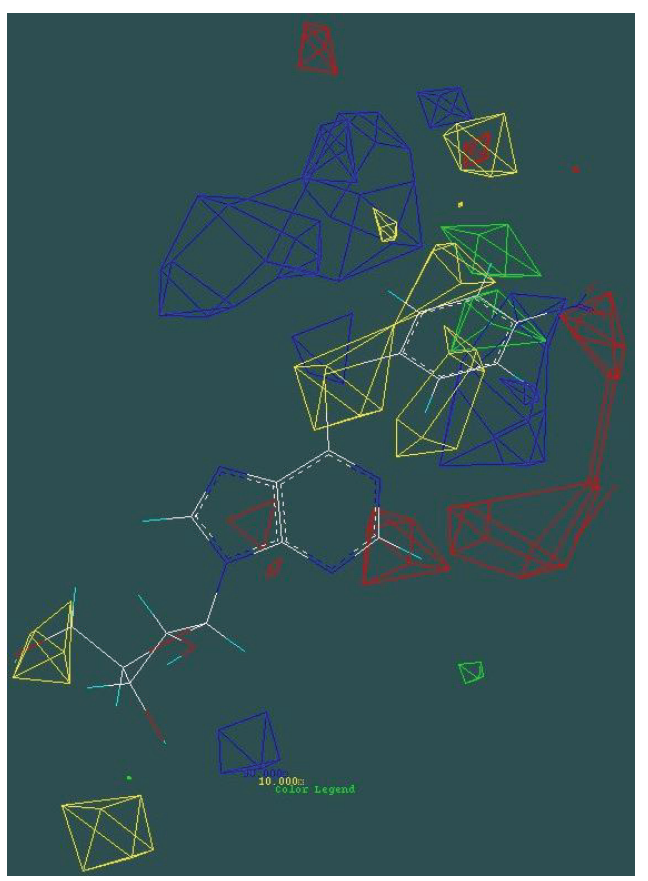

Figure A-6. NBMPR CoMFA hENT1 contours

Note: CoMFA color key. Steric: green- favored, yellow- disfavored; electrostatics: bluefavored, red- disfavored. 


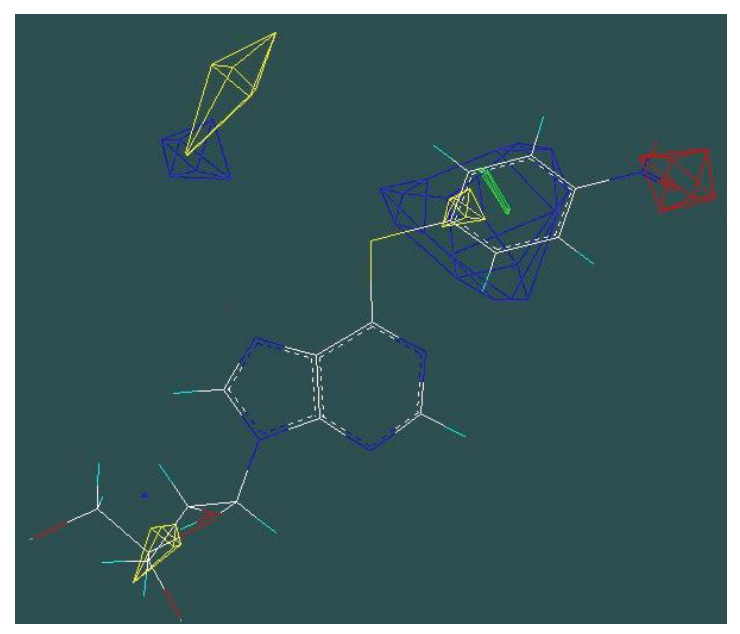

Figure A-7. NBMPR hENT1 CoMSIA steric and electrostatic contours

Note: CoMSIA color key. Steric: green- favored, yellow- disfavored; electrostatic: bluefavored, red-disfavored.

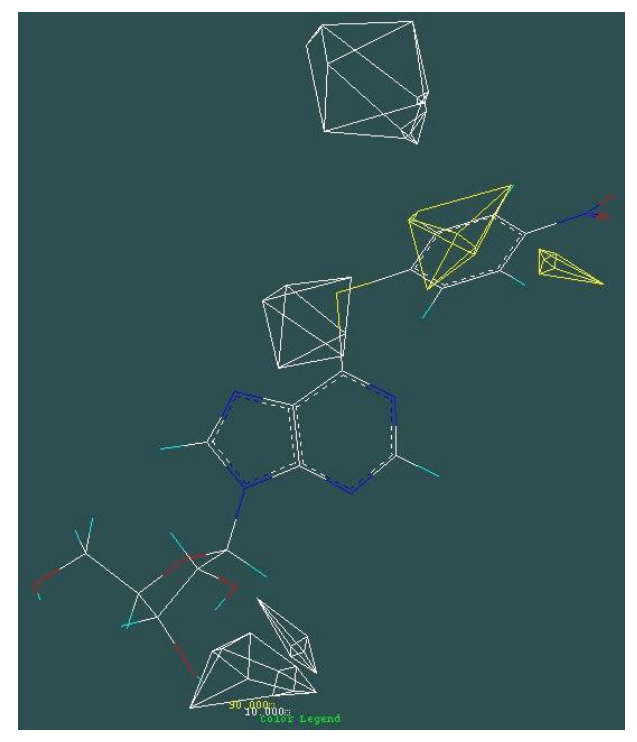

\section{Figure A-8. NBMPR hENT1 CoMSIA hydrophobic contours}

Note: CoMSIA color key. Hydrophobic: yellow- favored, white- disfavored. 


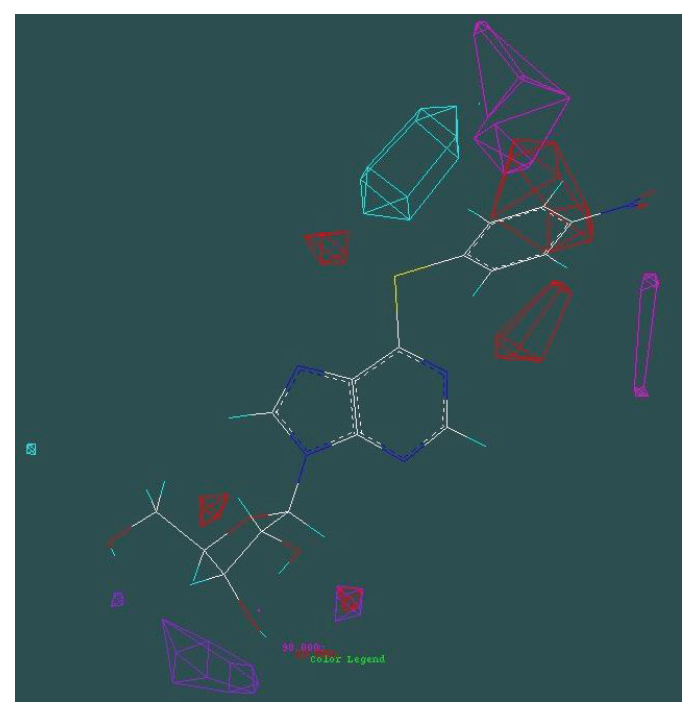

Figure A-9. NBMPR hENT1 CoMSIA hydrogen bond donor and acceptor contours

Note: CoMSIA color key. Hydrogen-bond donor: cyan- favored, purple- disfavored; hydrogen-bond acceptor: magenta- favored, red- disfavored.

\section{hENT4 Models}

\section{hENT4 Dipyridamole Models}

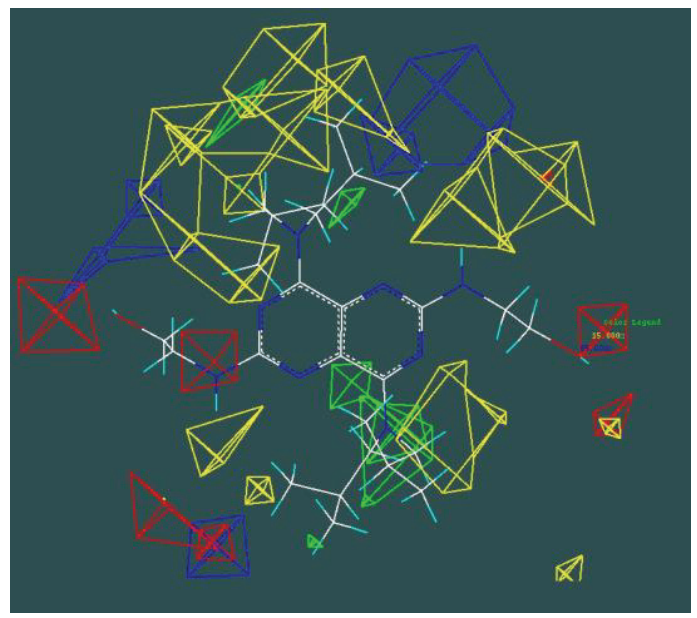

Figure A-10. Dipyridamole hENT4 CoMFA contours

Note: Color key. Steric: green- favored, yellow- disfavored; electrostatics: blue-favored, red- disfavored. 


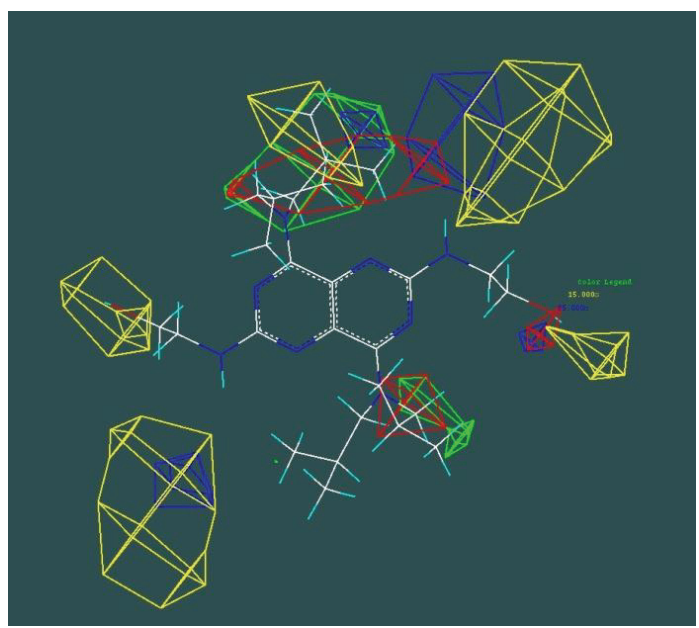

Figure A-11. Dipyridamole hENT4 CoMSIA steric and electrostatic contours

Note: Color key. Steric: green- favored, yellow- disfavored; electrostatic: blue- favored, red-disfavored.

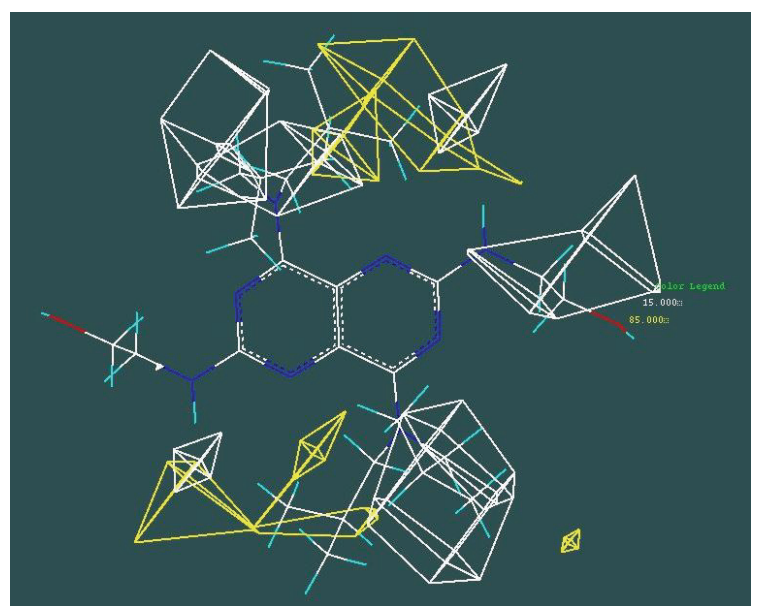

Figure A-12. Dipyridamole hENT4 CoMSIA hydrophobic contours

Note: Color key. Hydrophobic: yellow- favored, white- disfavored. 


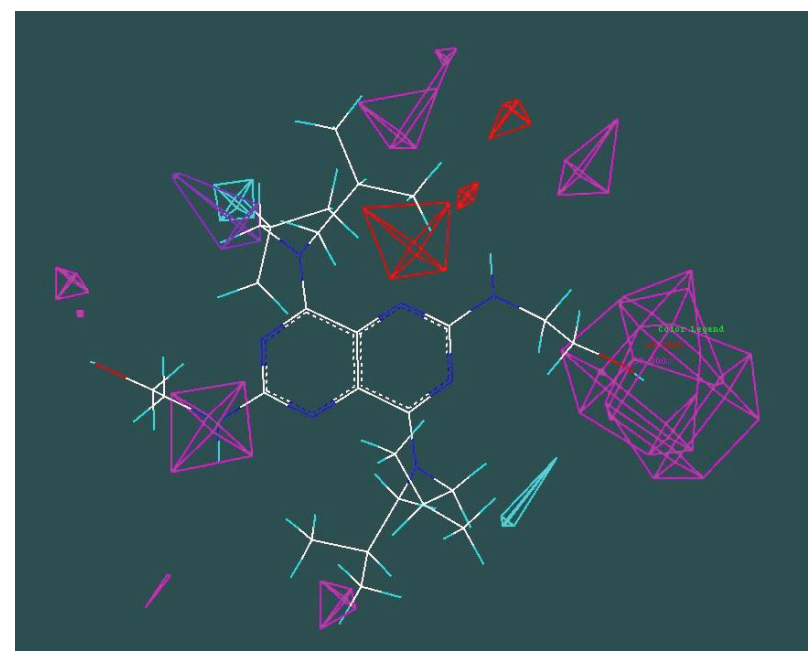

Figure A-13. Dipyridamole hENT4 CoMSIA hydrogen bond donor and acceptor contours

Note: Color key. Hydrogen-bond donor: cyan- favored, purple- disfavored; hydrogenbond acceptor: magenta- favored, red- disfavored.

\section{hENT4 Flavone Models}

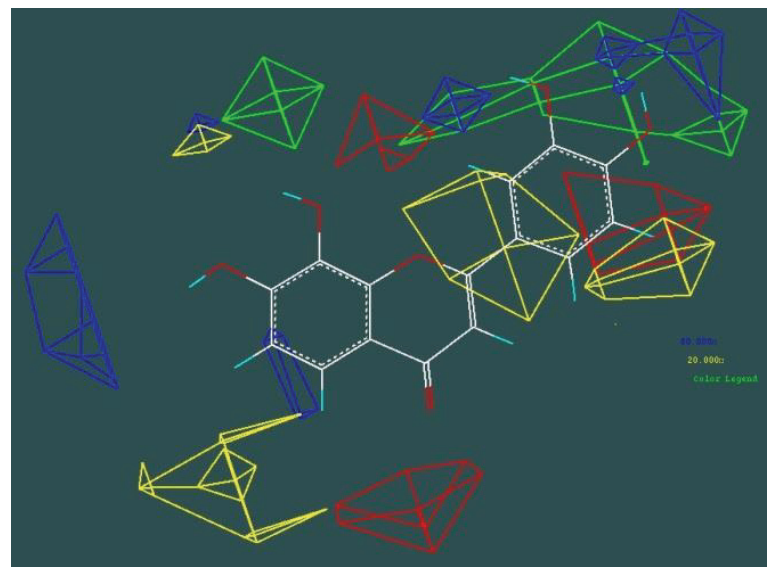

Figure A-14. Flavone hENT4 CoMFA contours

Note: Color key. Steric: green- favored, yellow- disfavored; electrostatics: blue-favored, red- disfavored. 


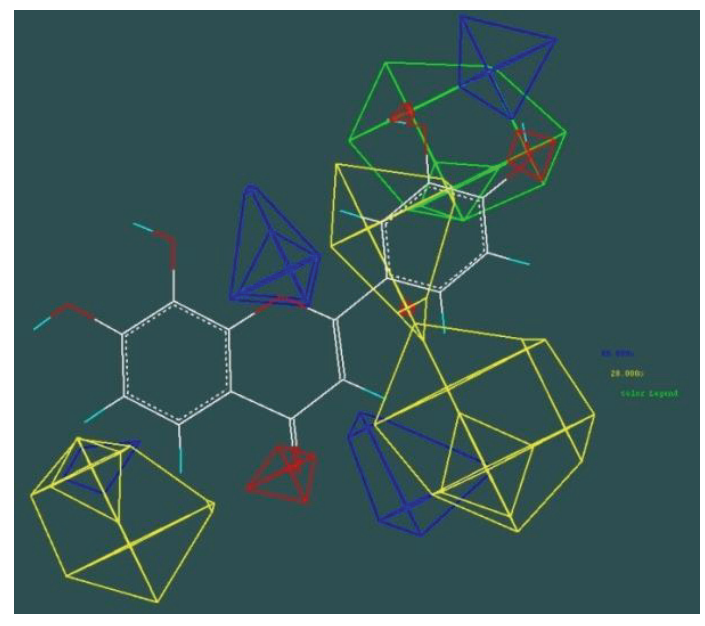

Figure A-15. Flavone hENT4 CoMSIA steric and electrostatic contours

Note: Color key. Steric: green- favored, yellow- disfavored; electrostatic: blue- favored, red-disfavored.

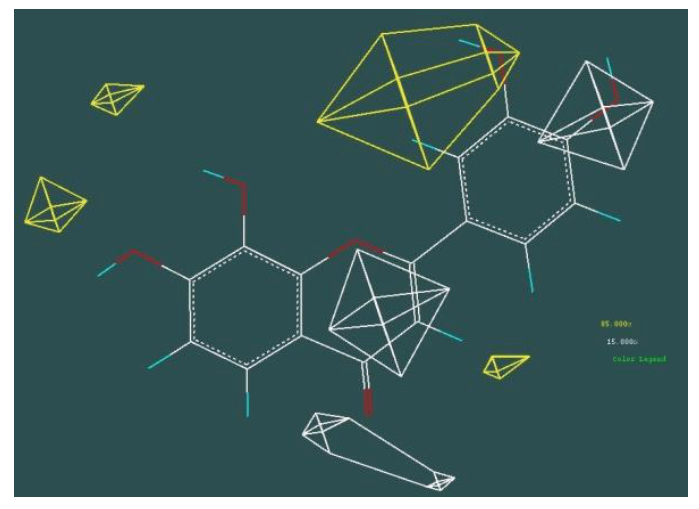

Figure A-16. Flavone hENT4 CoMSIA hydrophobic contours

Note: Color key. Hydrophobic: yellow- favored, white- disfavored. 


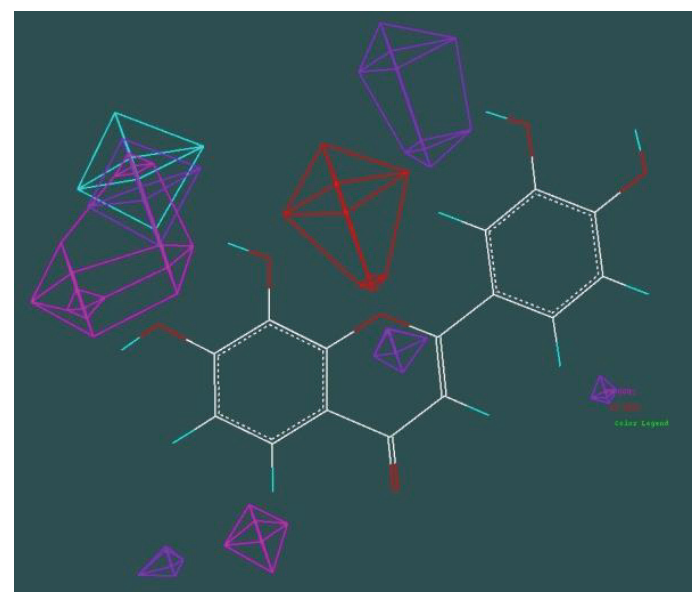

Figure A-17. Flavone hENT4 CoMSIA hydrogen bond donor and acceptor contours

Note: Color key. Hydrogen-bond donor: cyan- favored, purple- disfavored; hydrogenbond acceptor: magenta- favored, red- disfavored. 


\section{Chemistry Data}

\section{Mass Spectra}

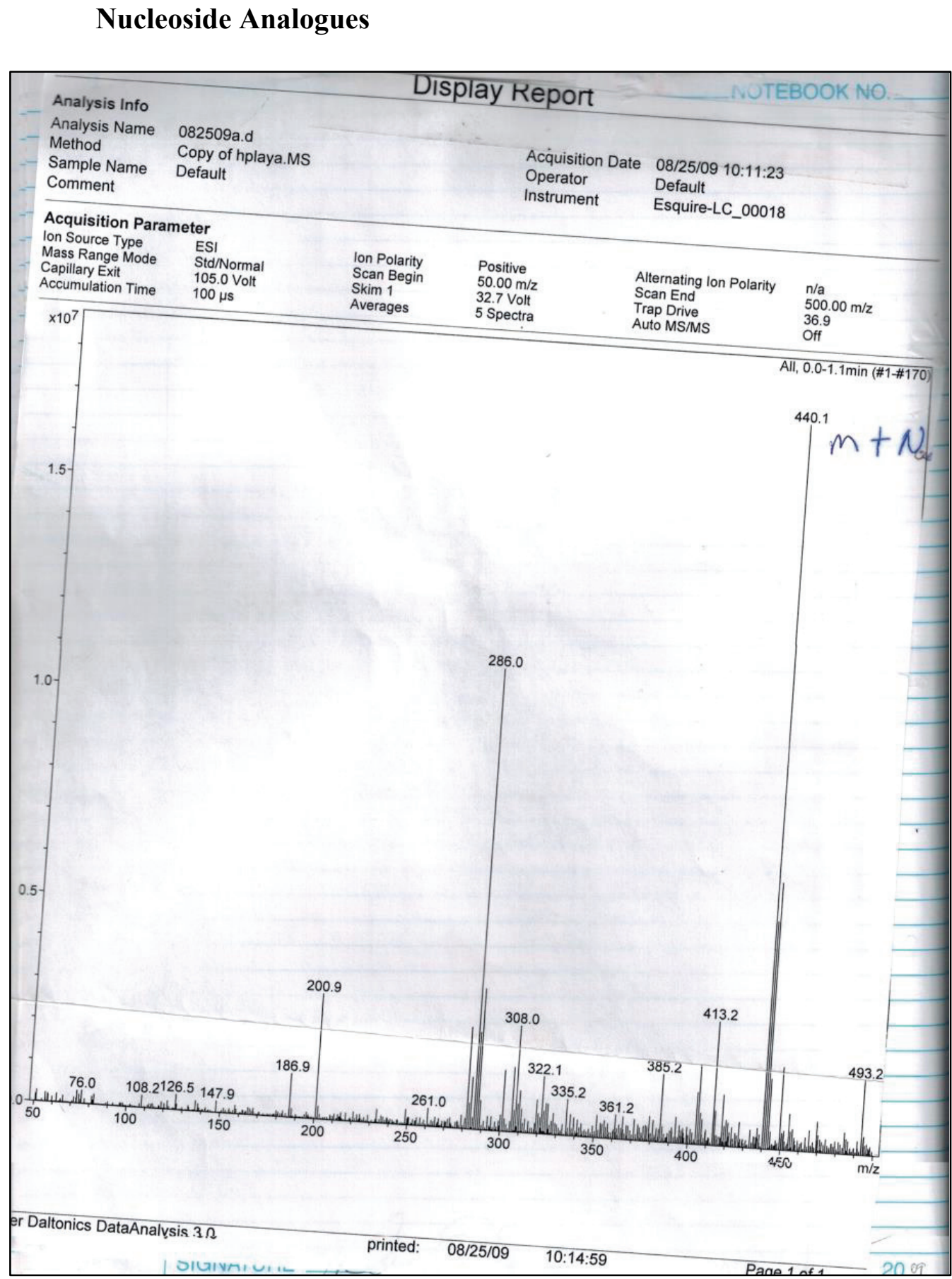

Figure A-18. HCPN01 mass spectra 


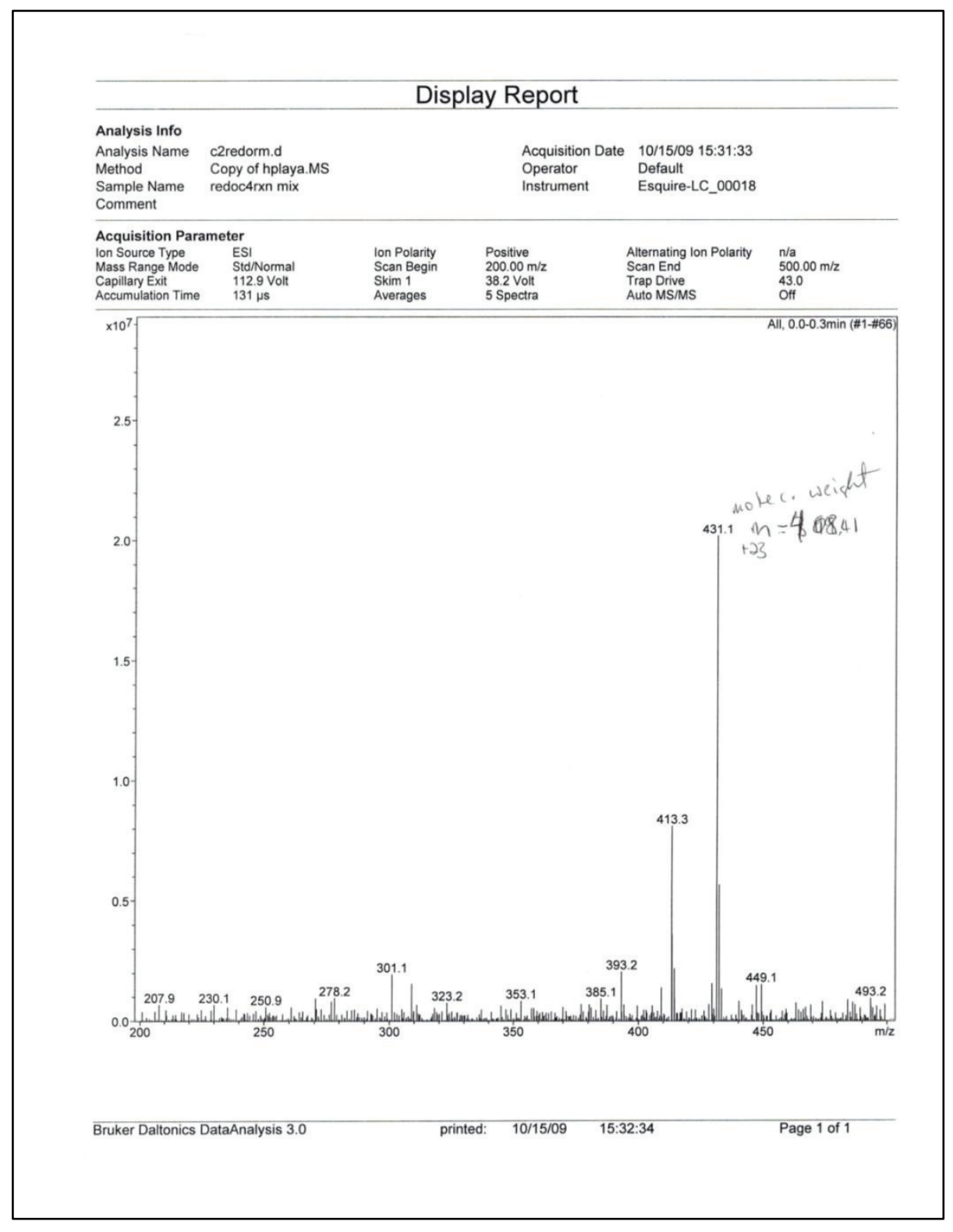

Figure A-19. HCPN05 mass spectra 


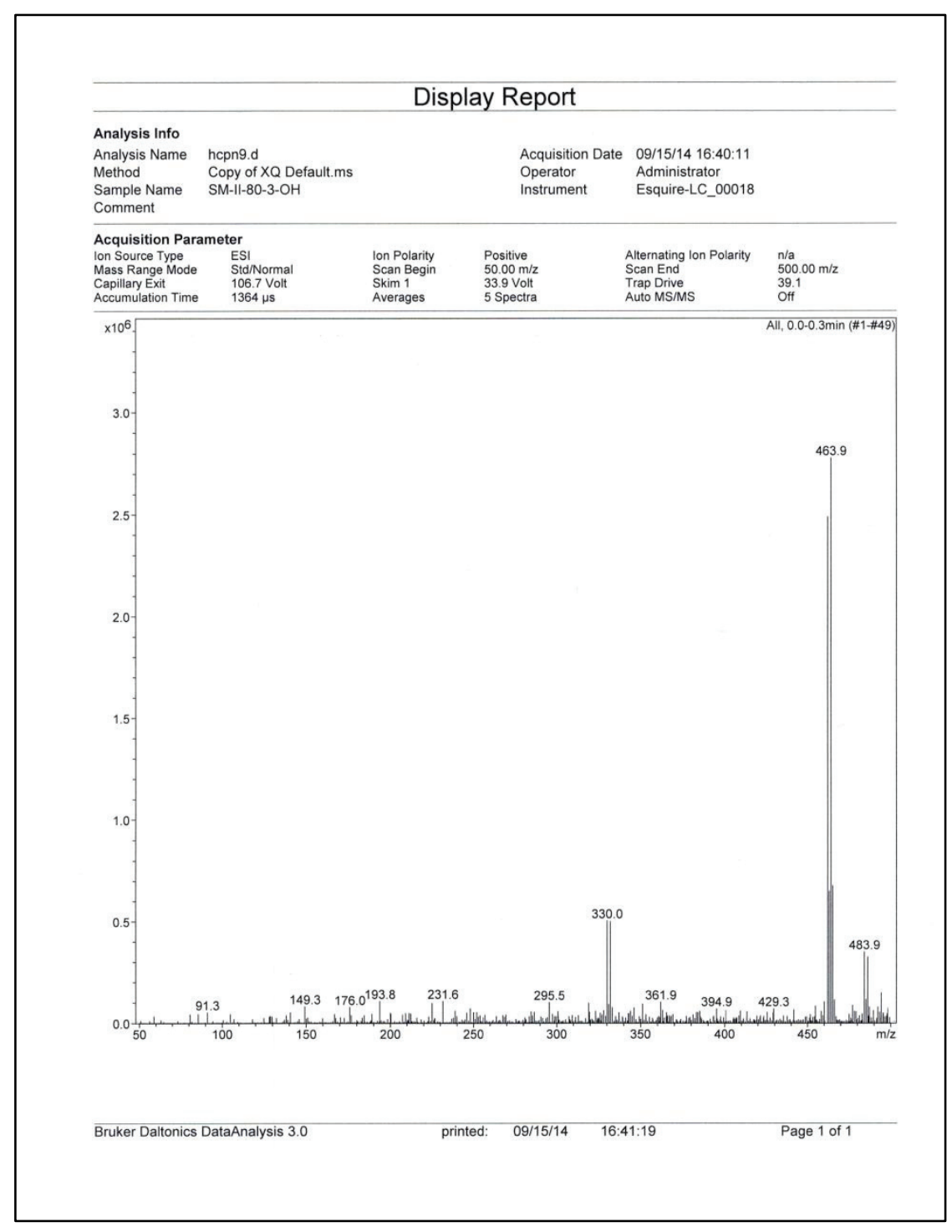

Figure A-20. HCP09 mass spectra 


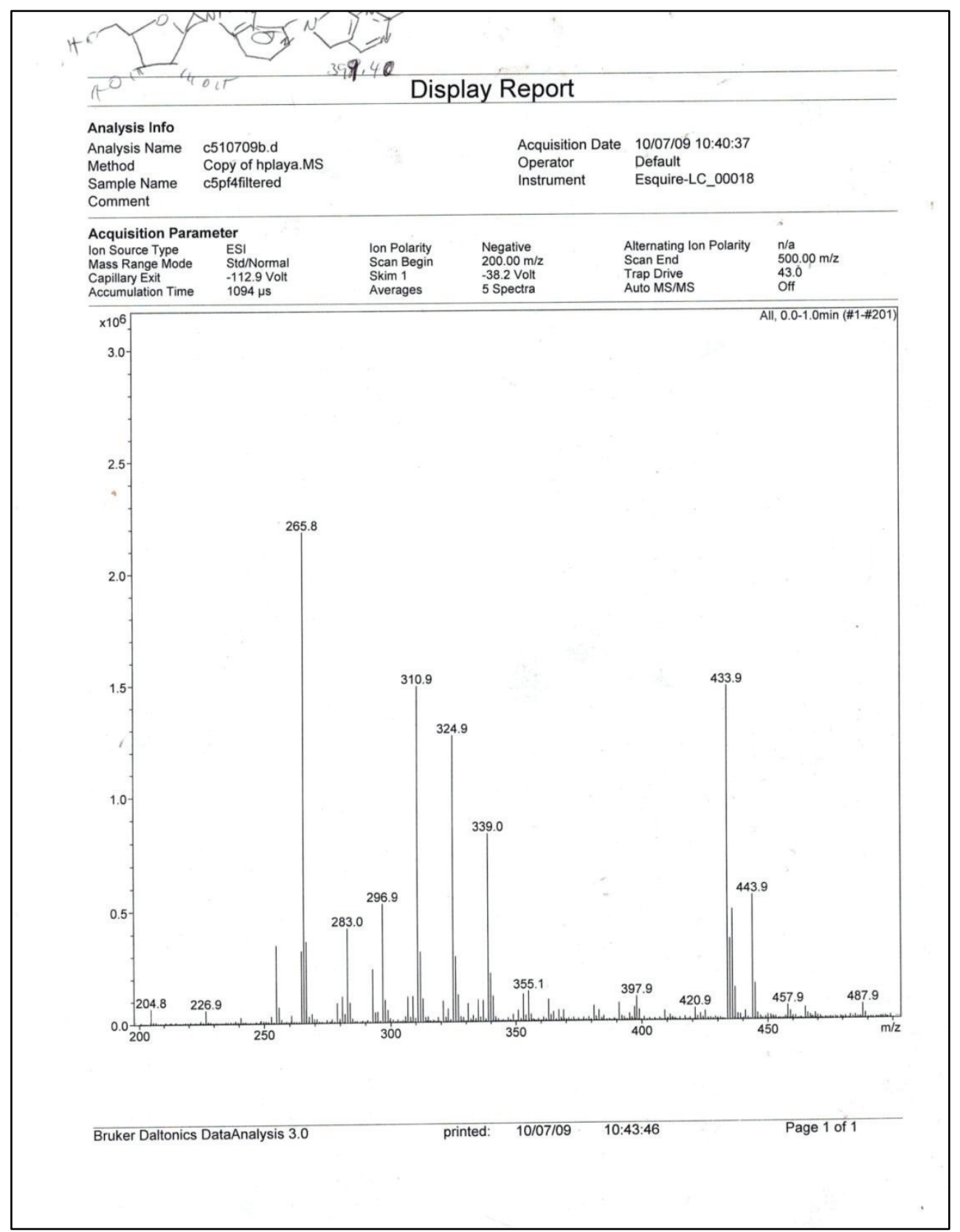

Figure A-21. HCPN22 mass spectra 


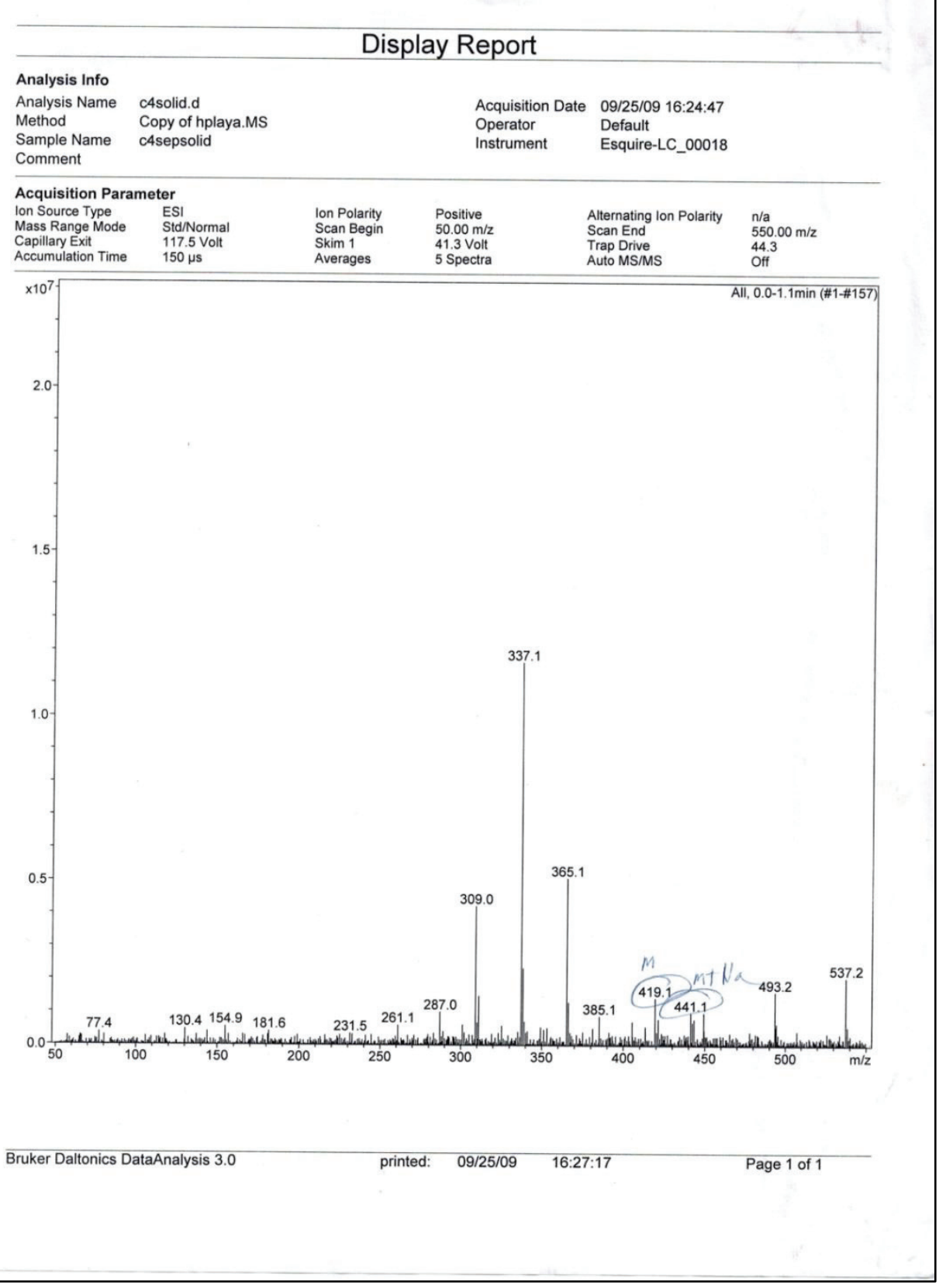

Figure A-22. HCPN29 mass spectra 


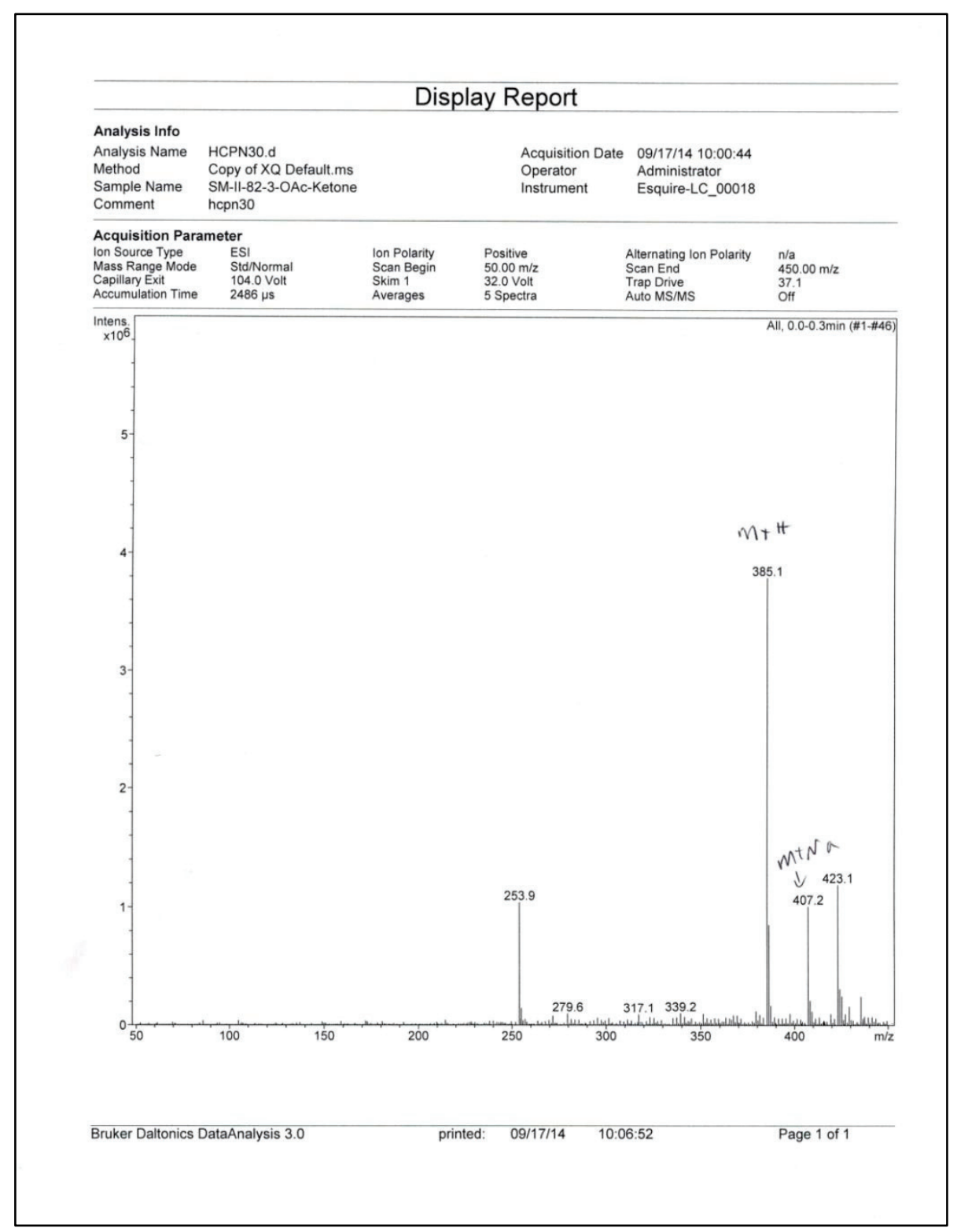

Figure A-23. HCPN30 mass spectra 


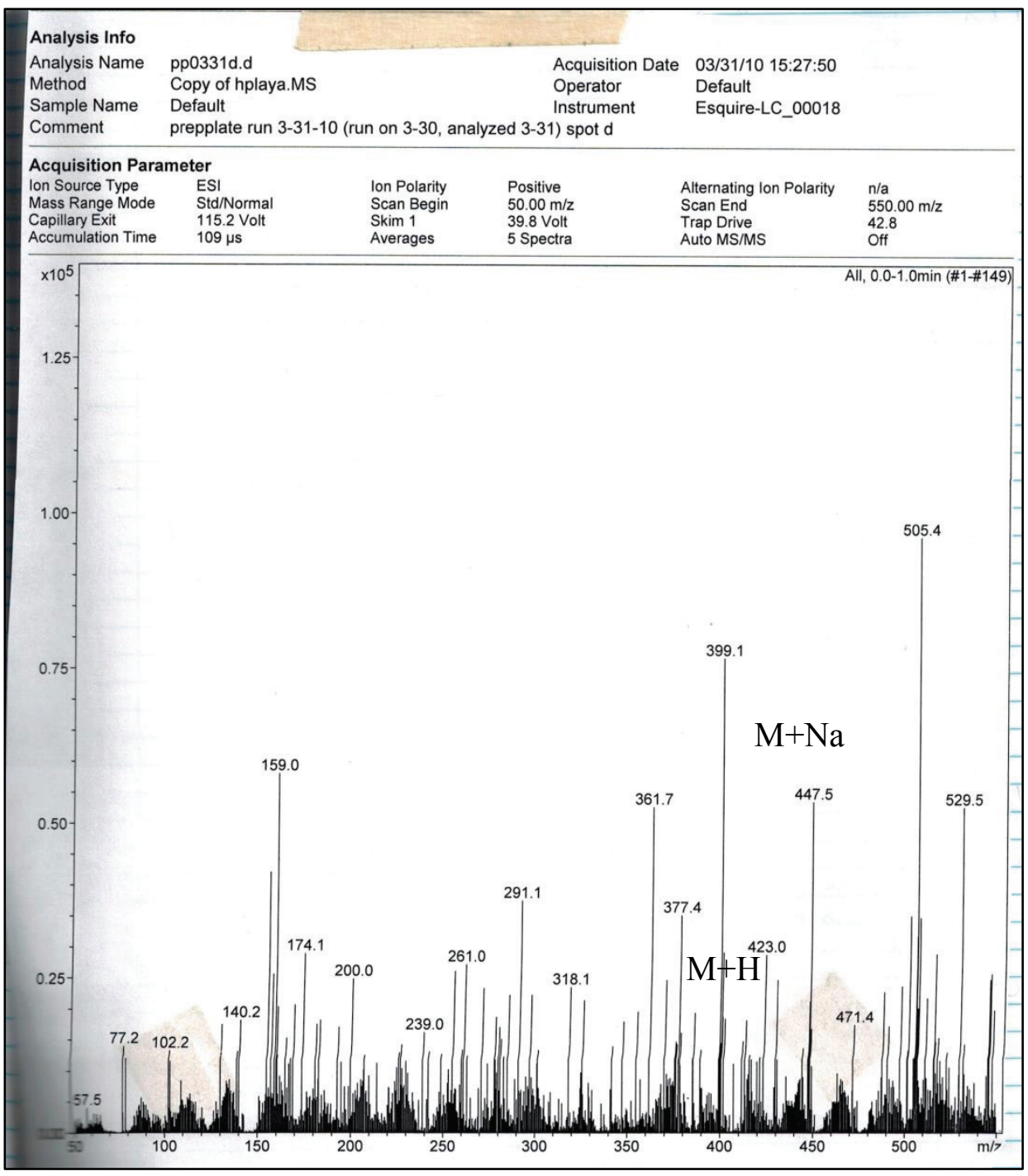

Figure A-24. HCPN34 mass spectra 


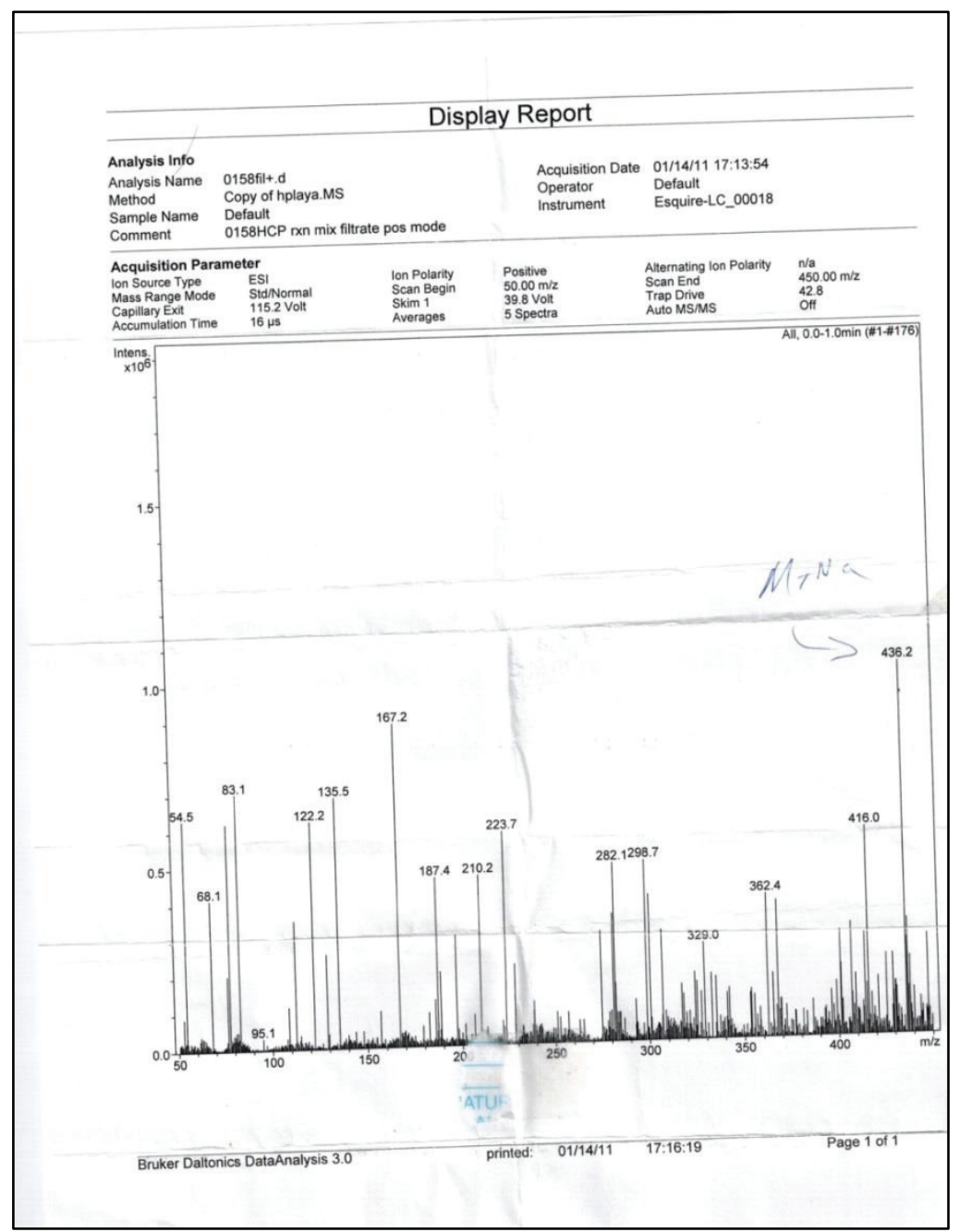

Figure A-25. HCPN58 mass spectra 


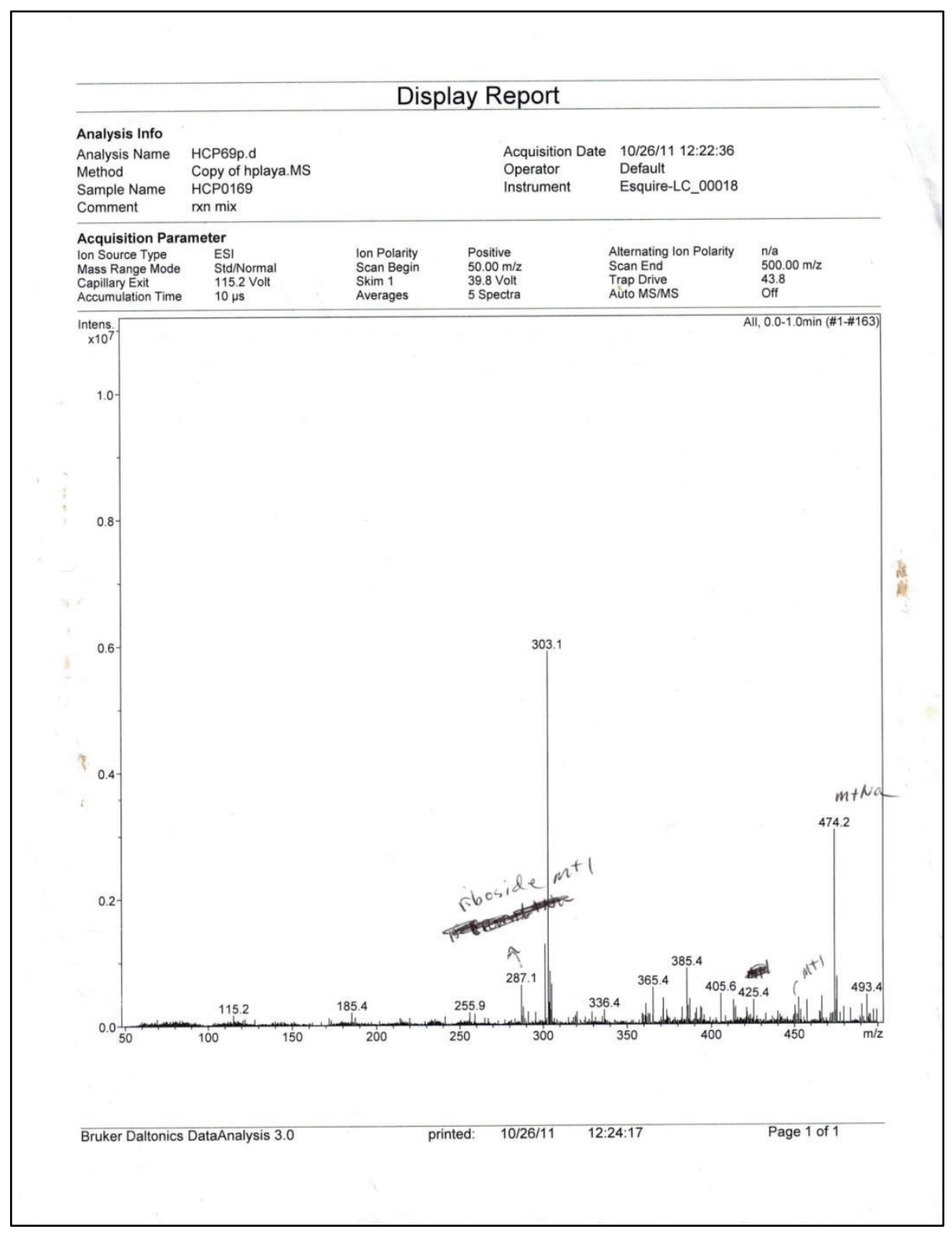

Figure A-26. HCPN69 mass spectra 


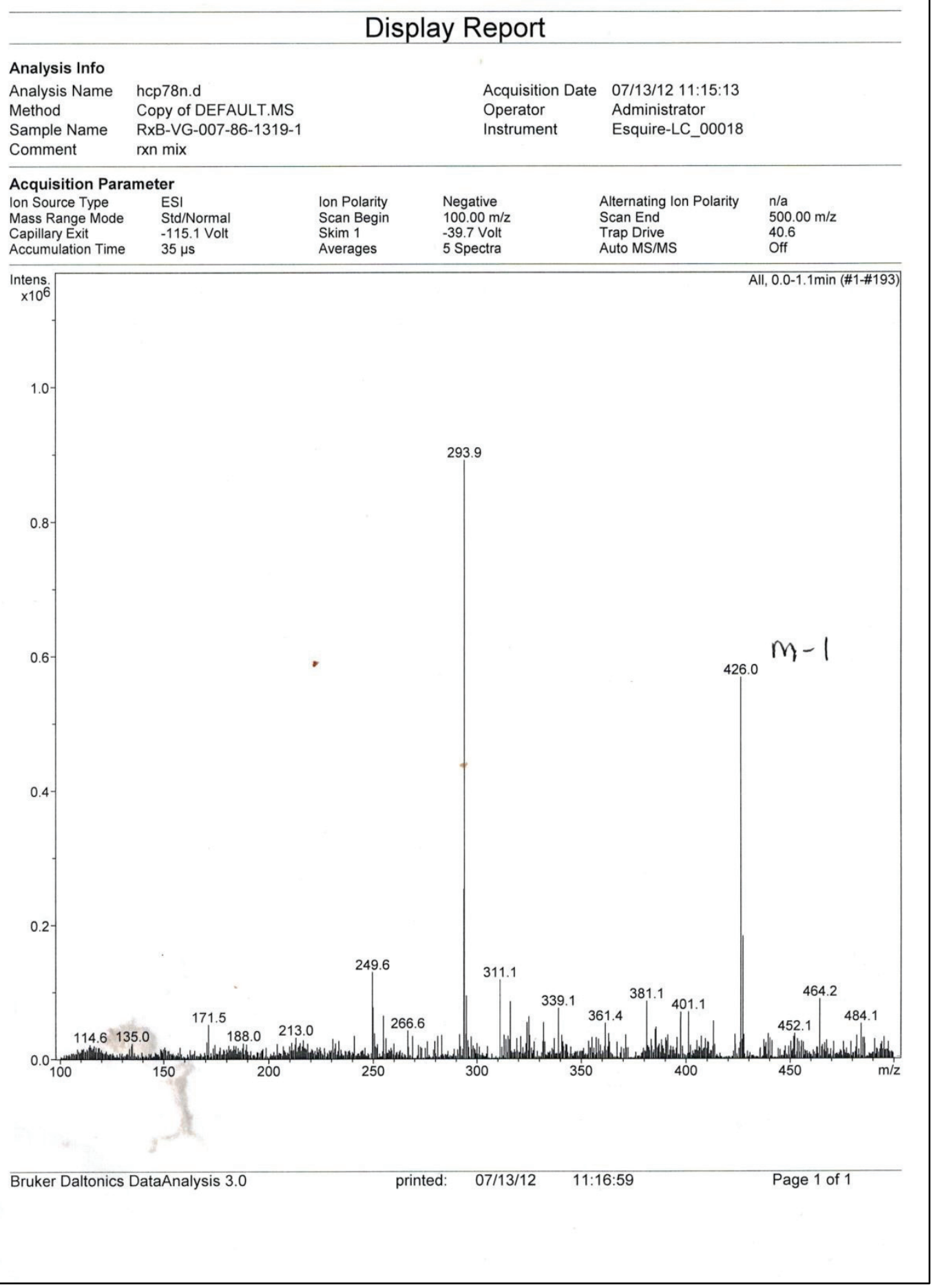

Figure A-27. HCPN78 mass spectra 


\section{Pyridone Analogues}

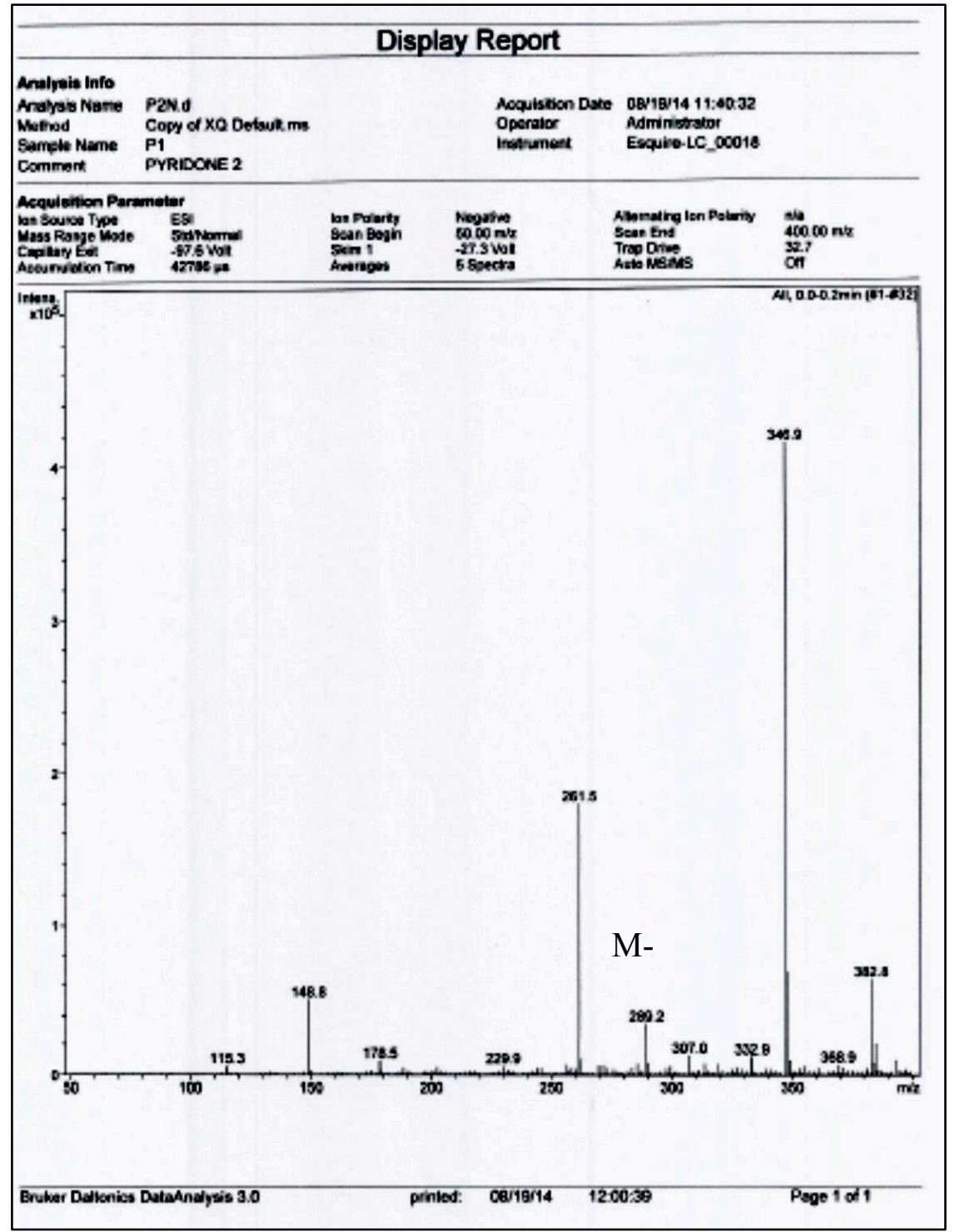

Figure A-28. Pyr2 mass spectra 


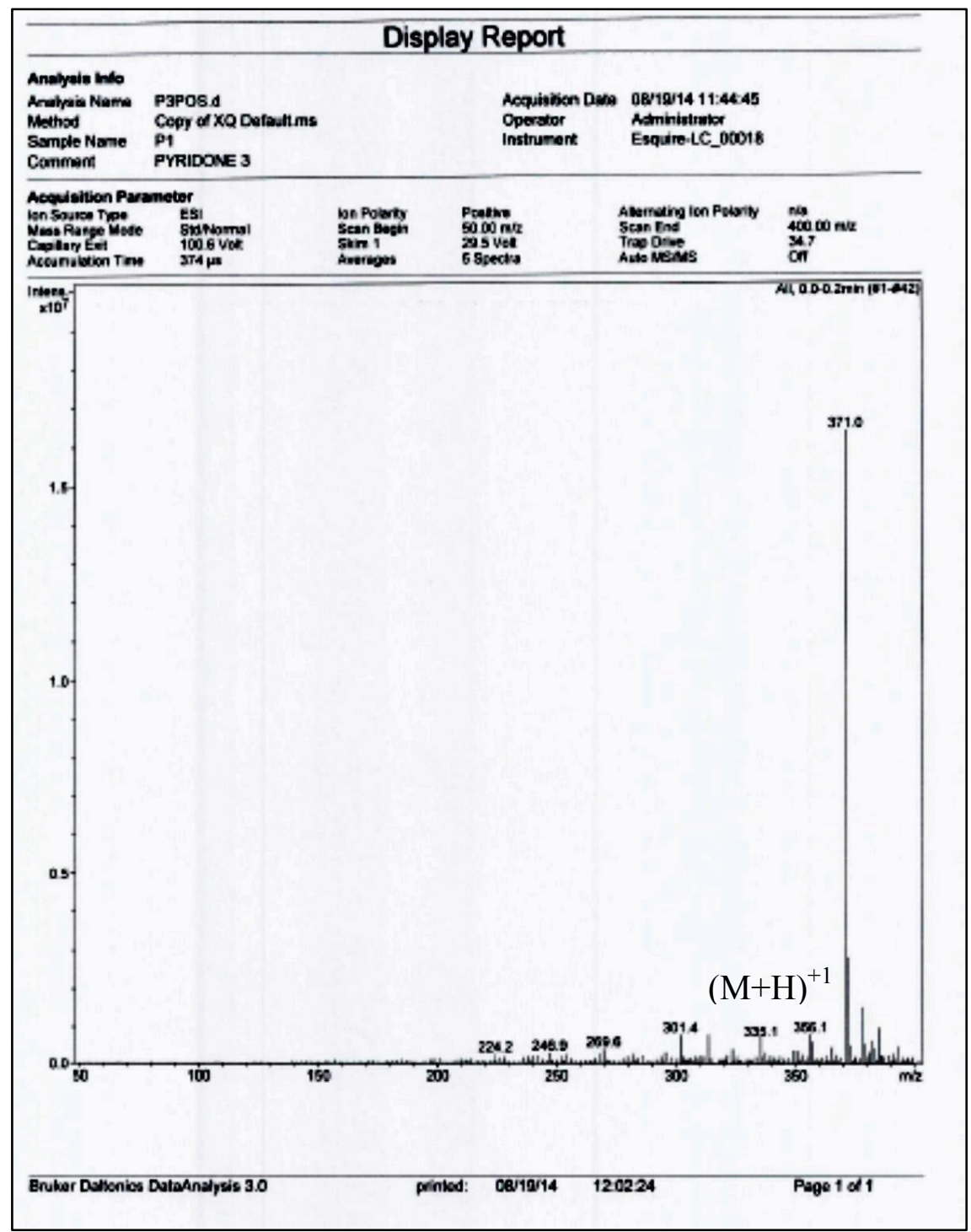

Figure A-29. Pyr3 mass spectra 


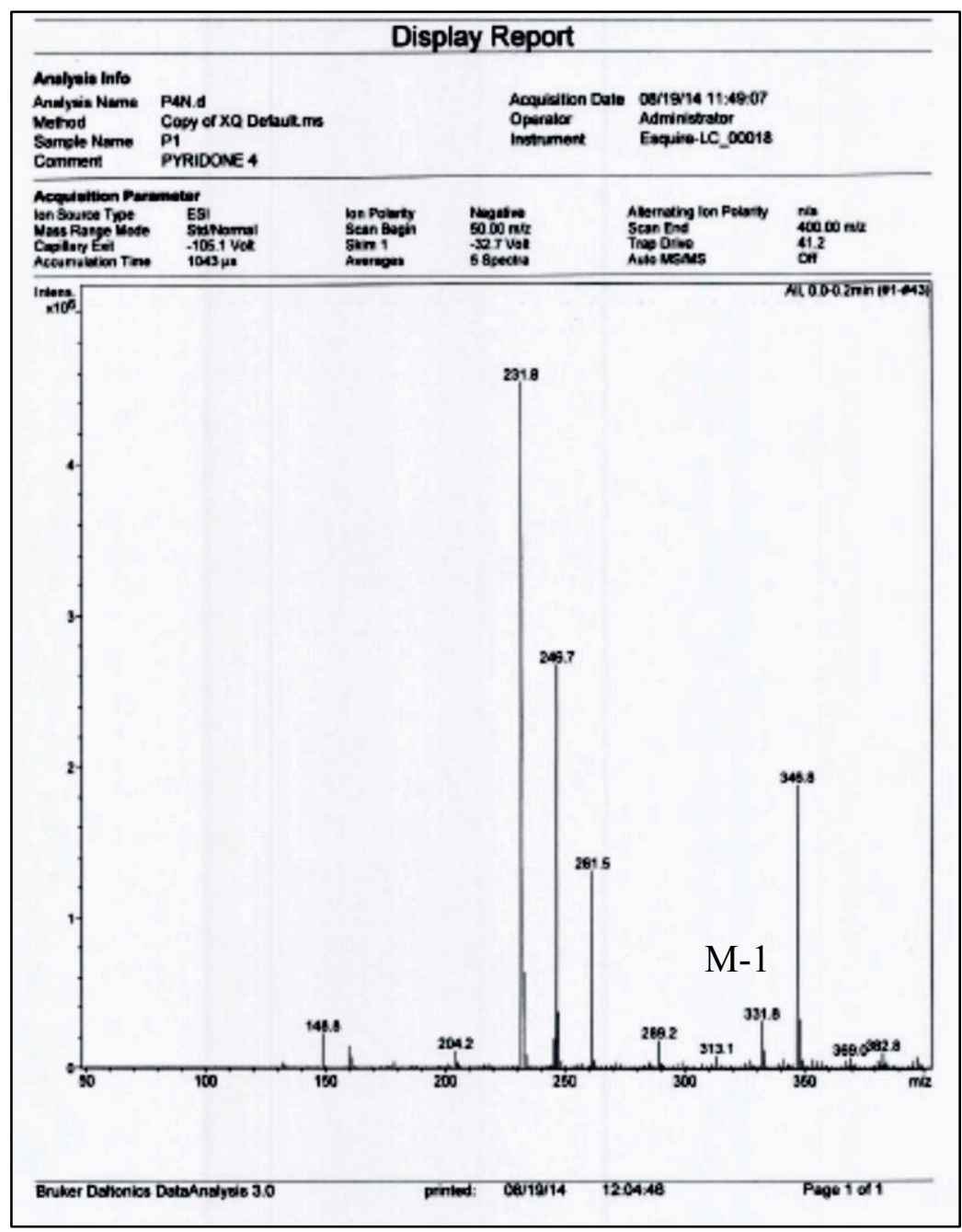

Figure A-30. Pyr4 mass spectra 


\section{NMR Spectra}

\section{Nucleoside Analogues}

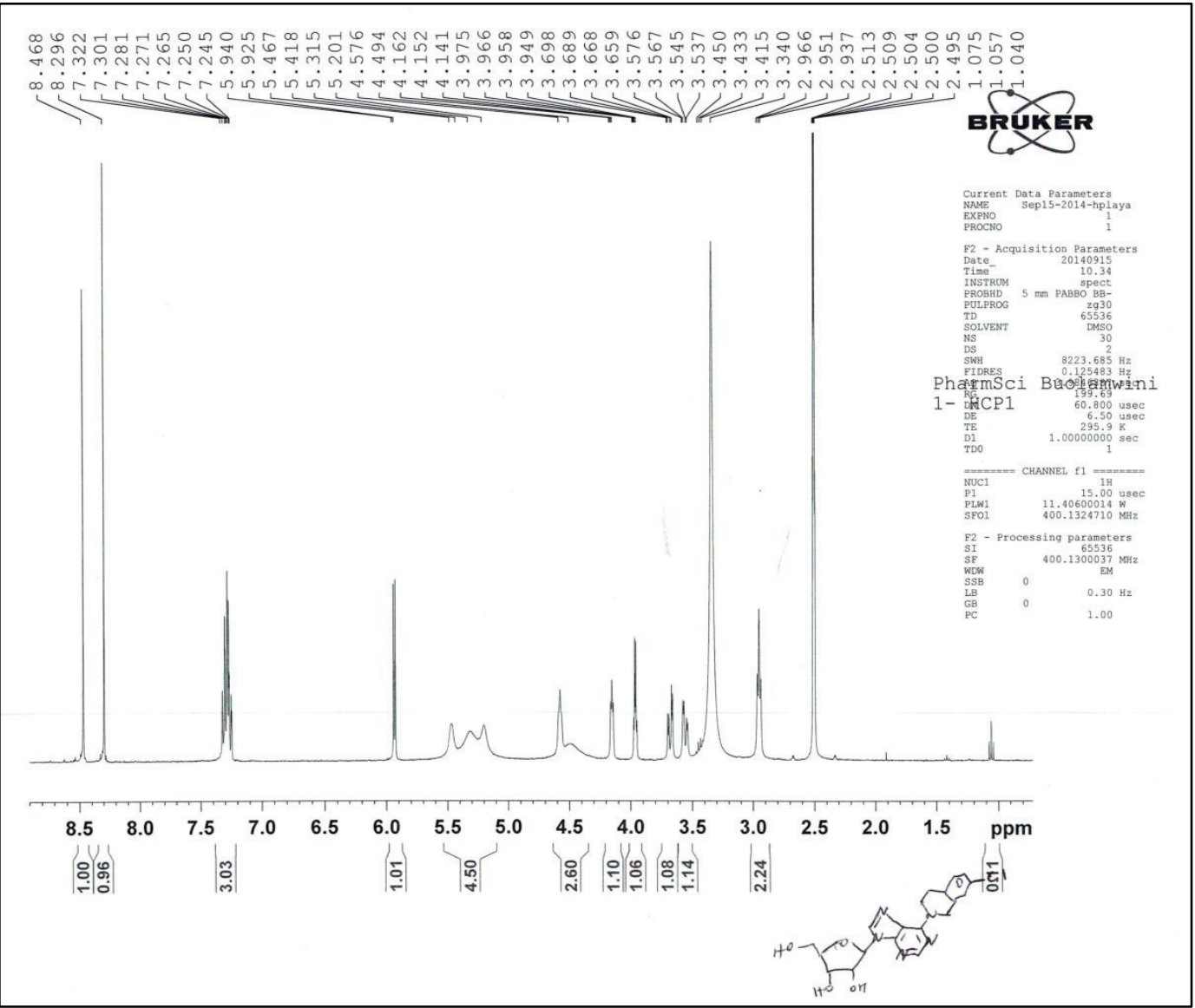

Figure A-31. HCPN01 proton NMR 


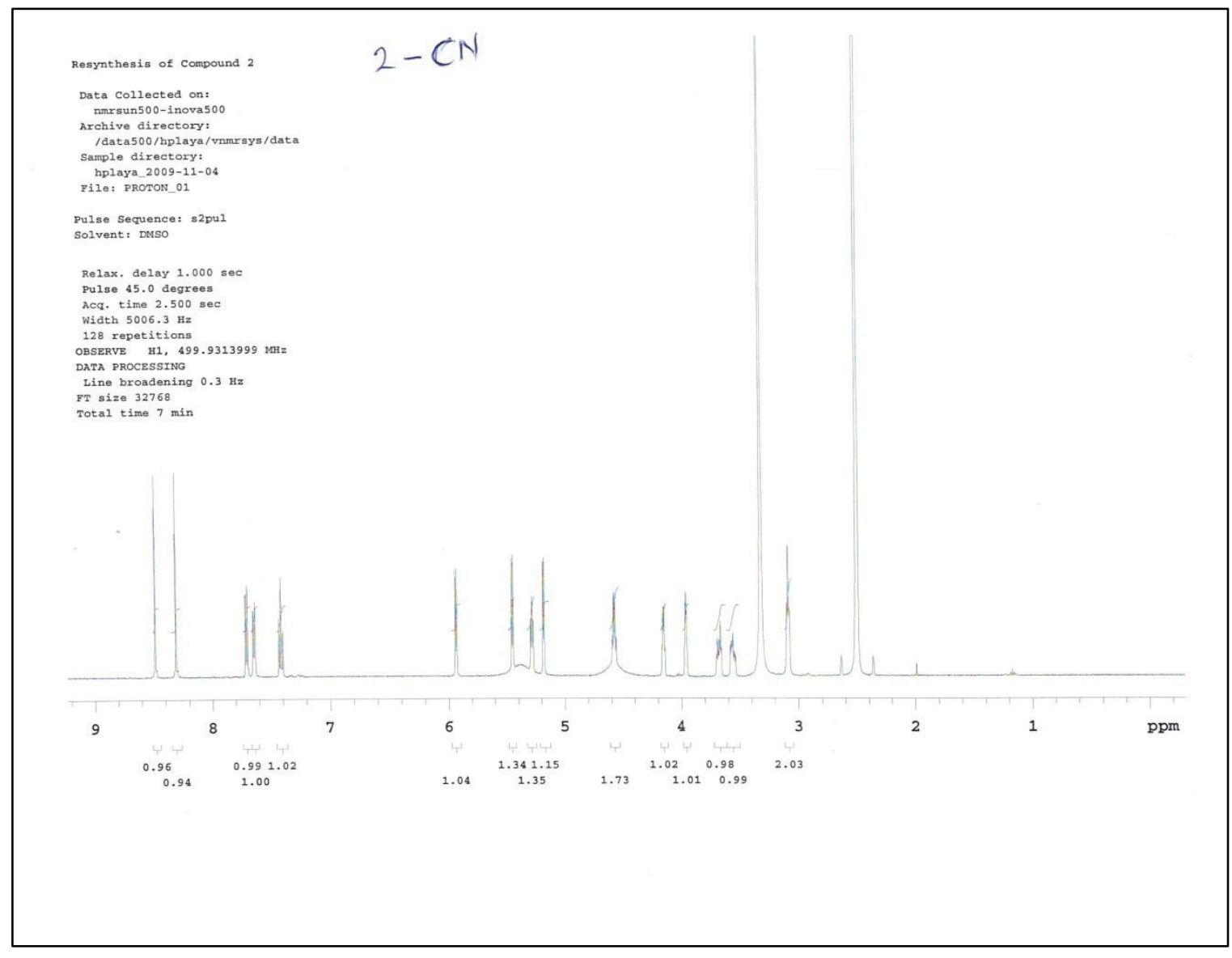

Figure A-32. HCPN05 proton NMR 


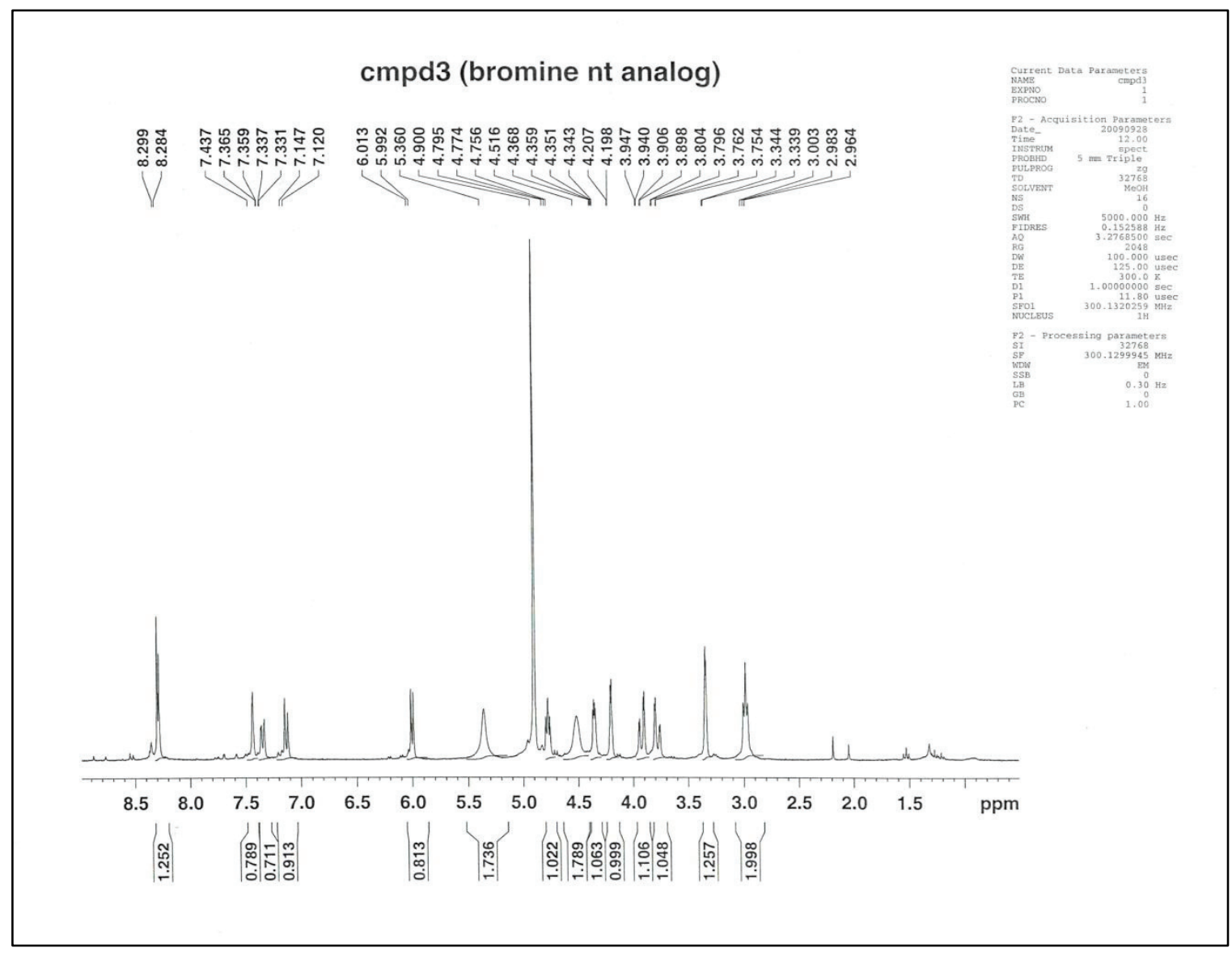

Figure A-33. HCPN09 proton NMR 


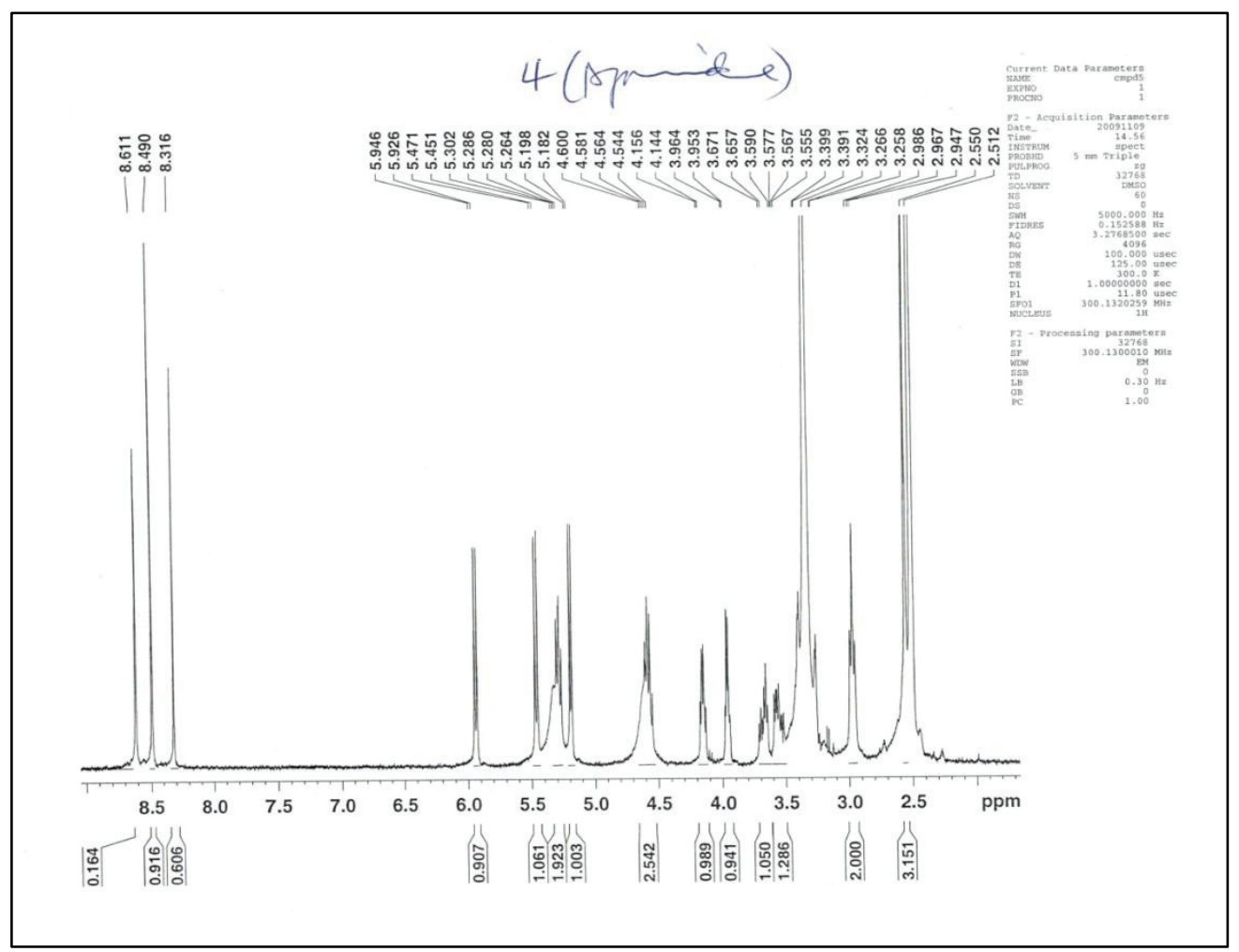

Figure A-34. HCPN22 proton NMR 


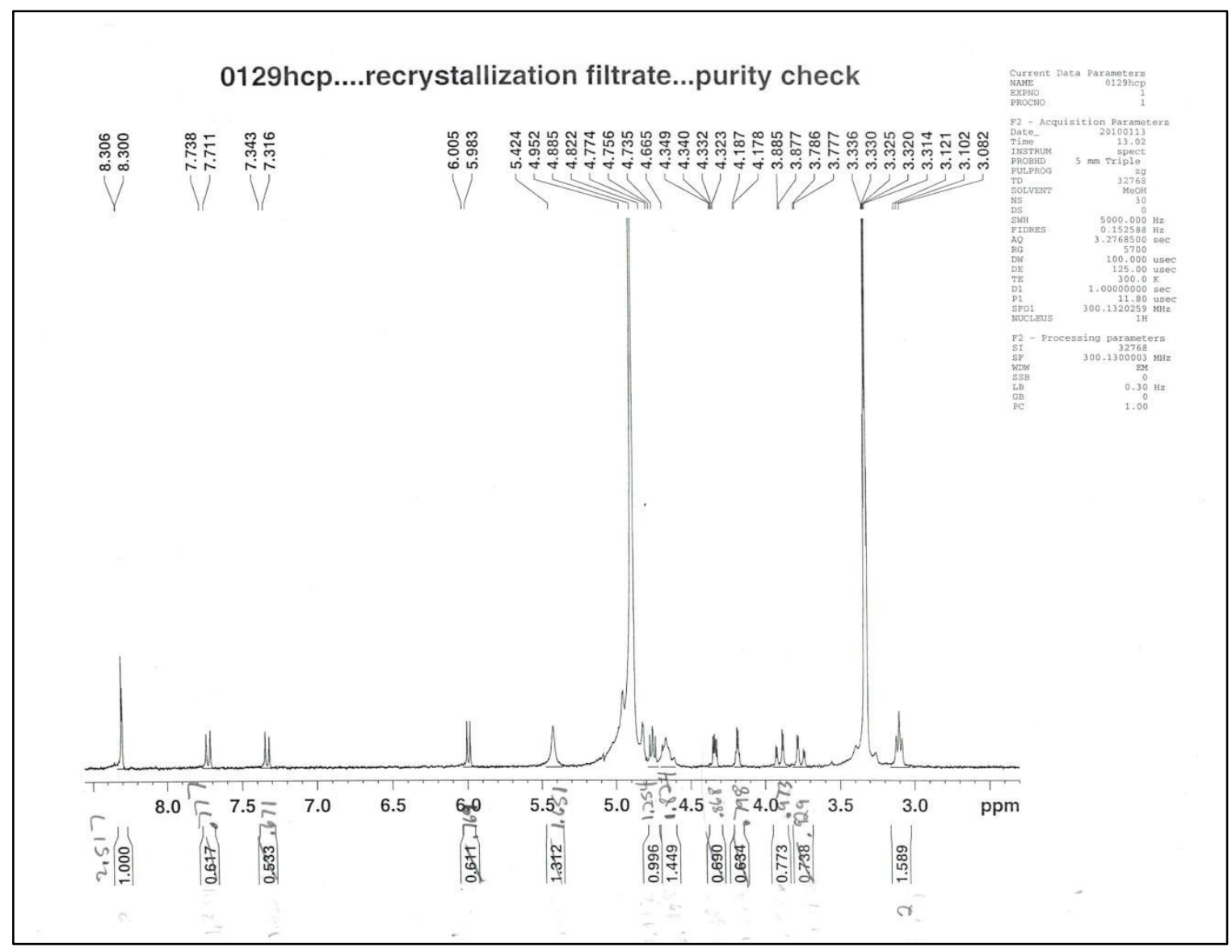

Figure A-35. HCPN29 proton NMR 


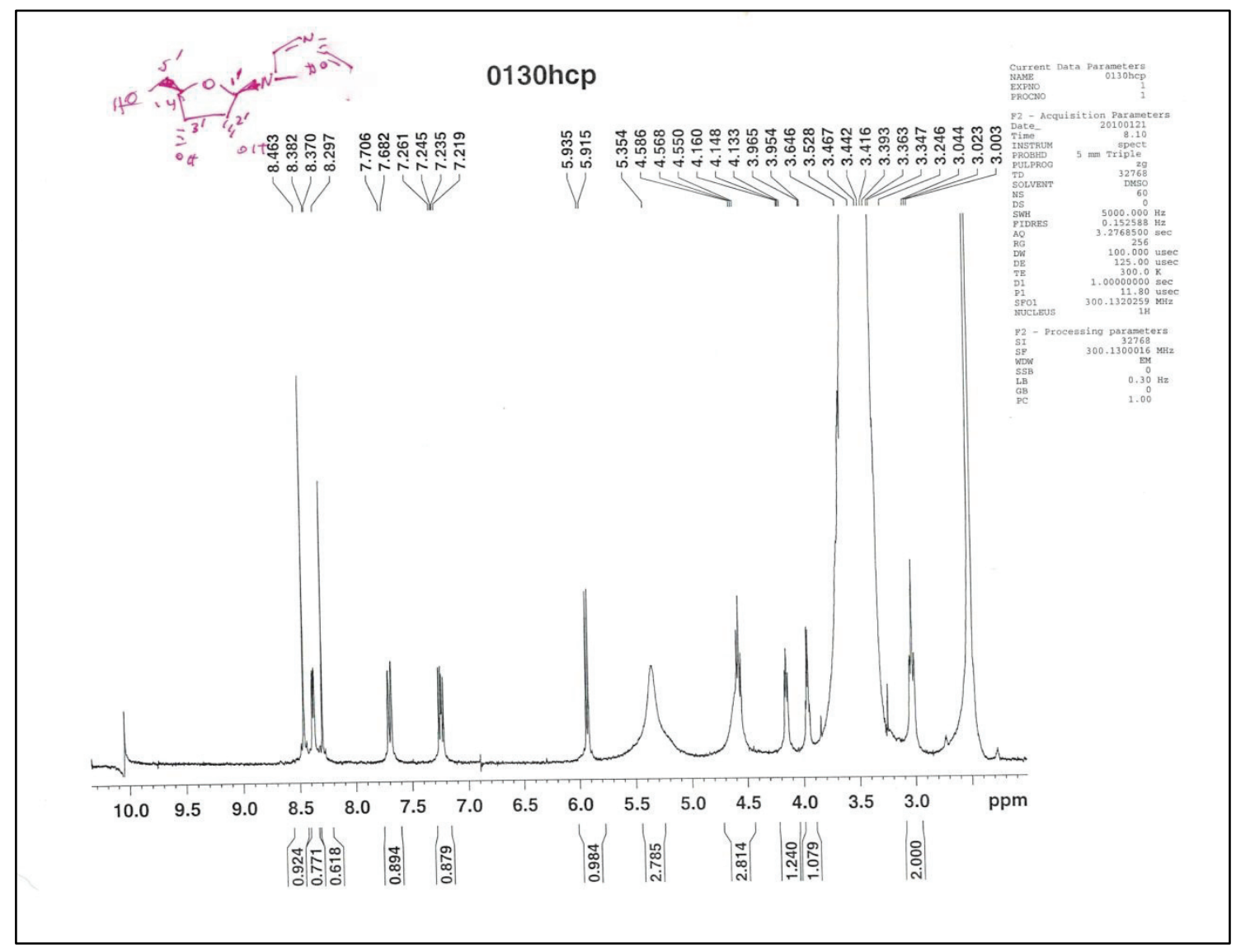

Figure A-36. HCPN30 proton NMR 


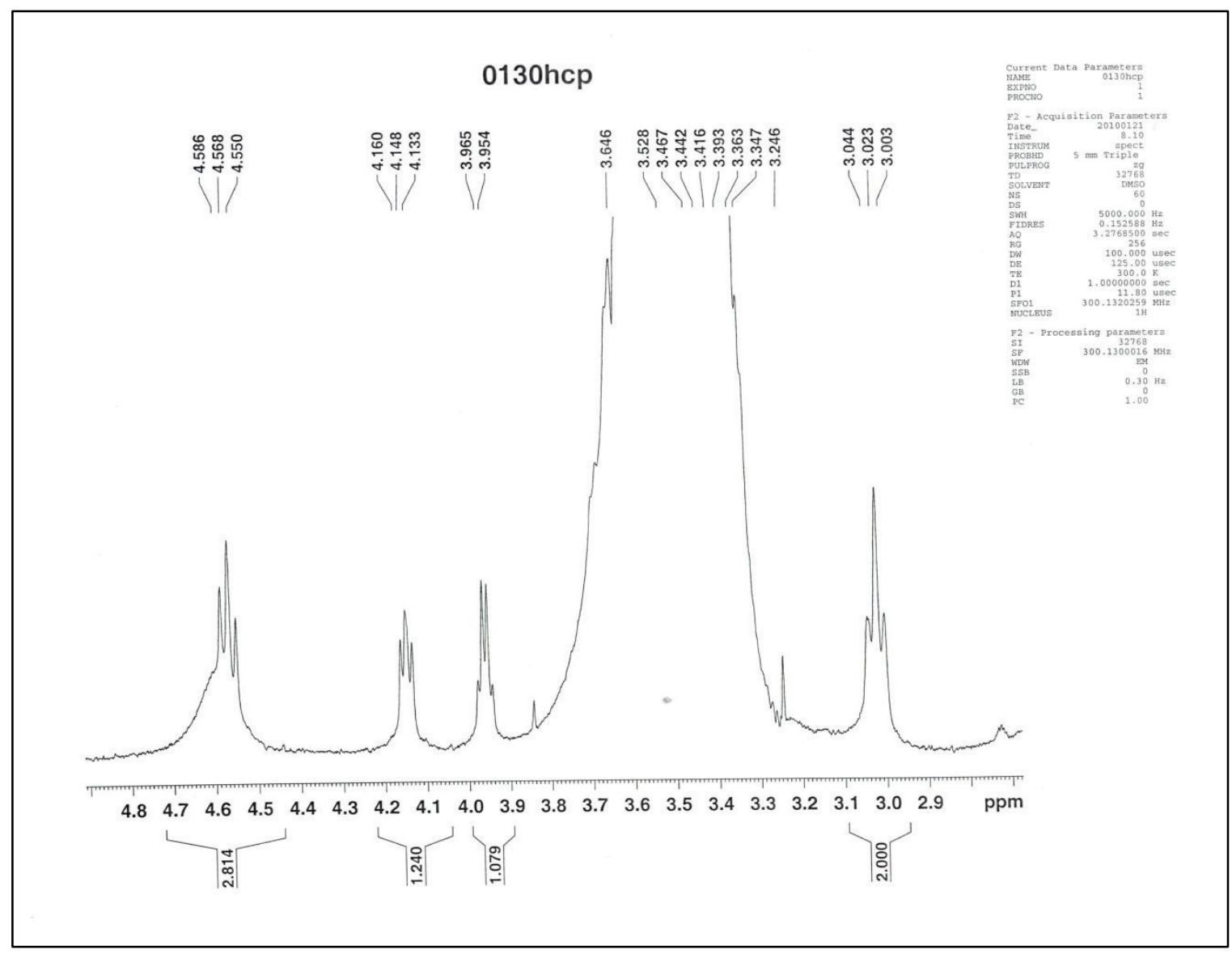

Figure A-37. Expanded upfield section HCPN30 NMR 


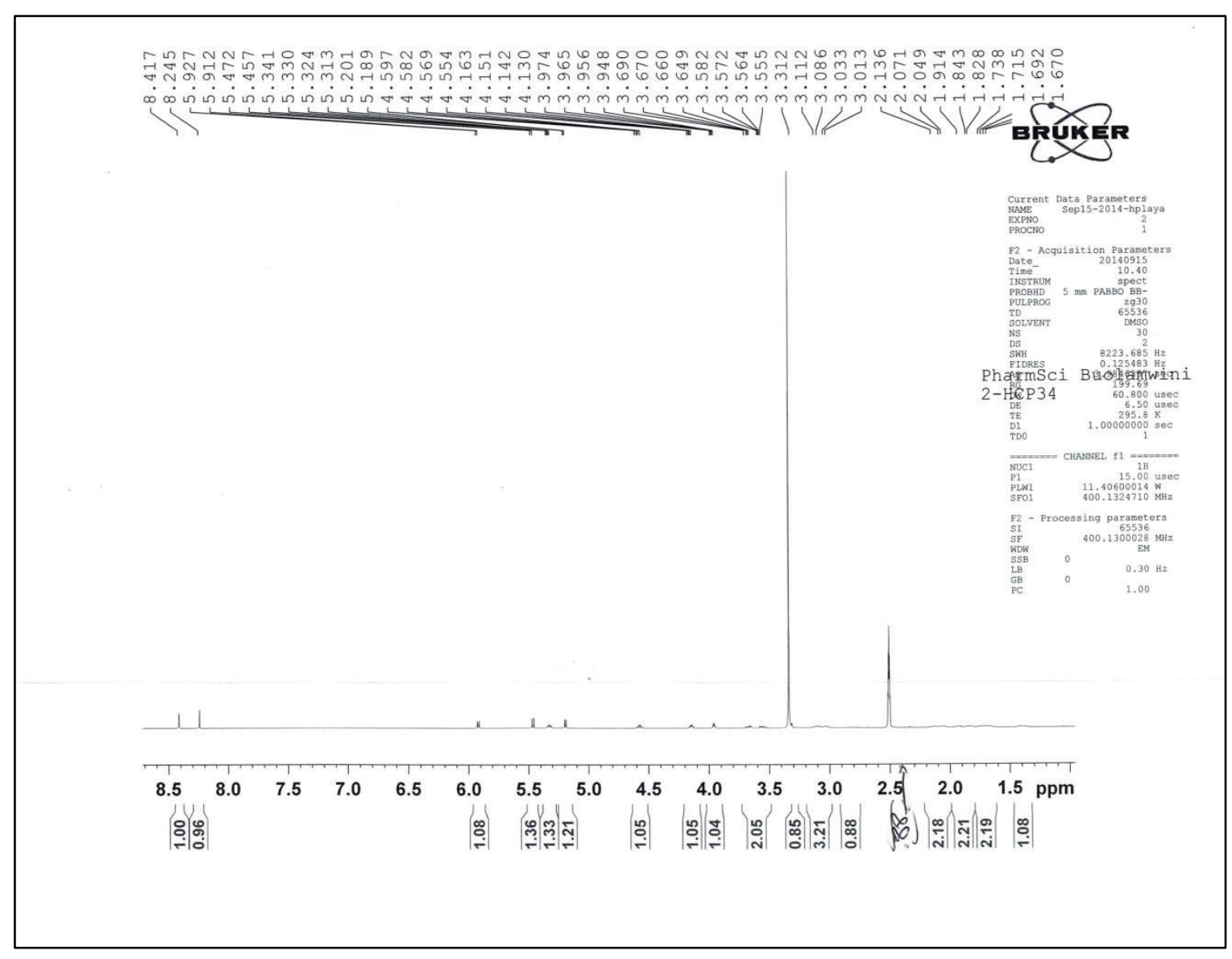

Figure A-38. HCPN34 proton NMR 


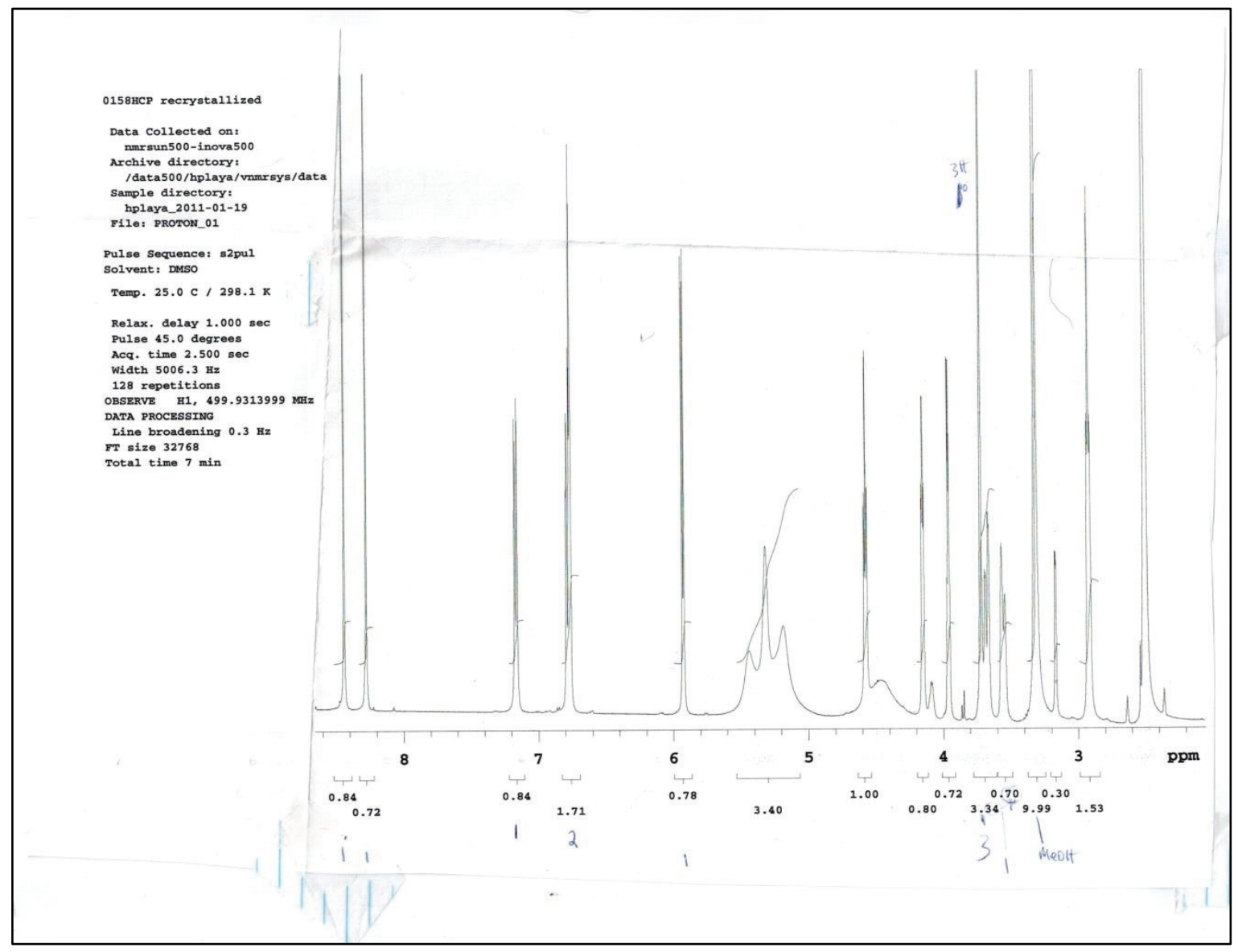

Figure A-39. HCPN58 expanded proton NMR 


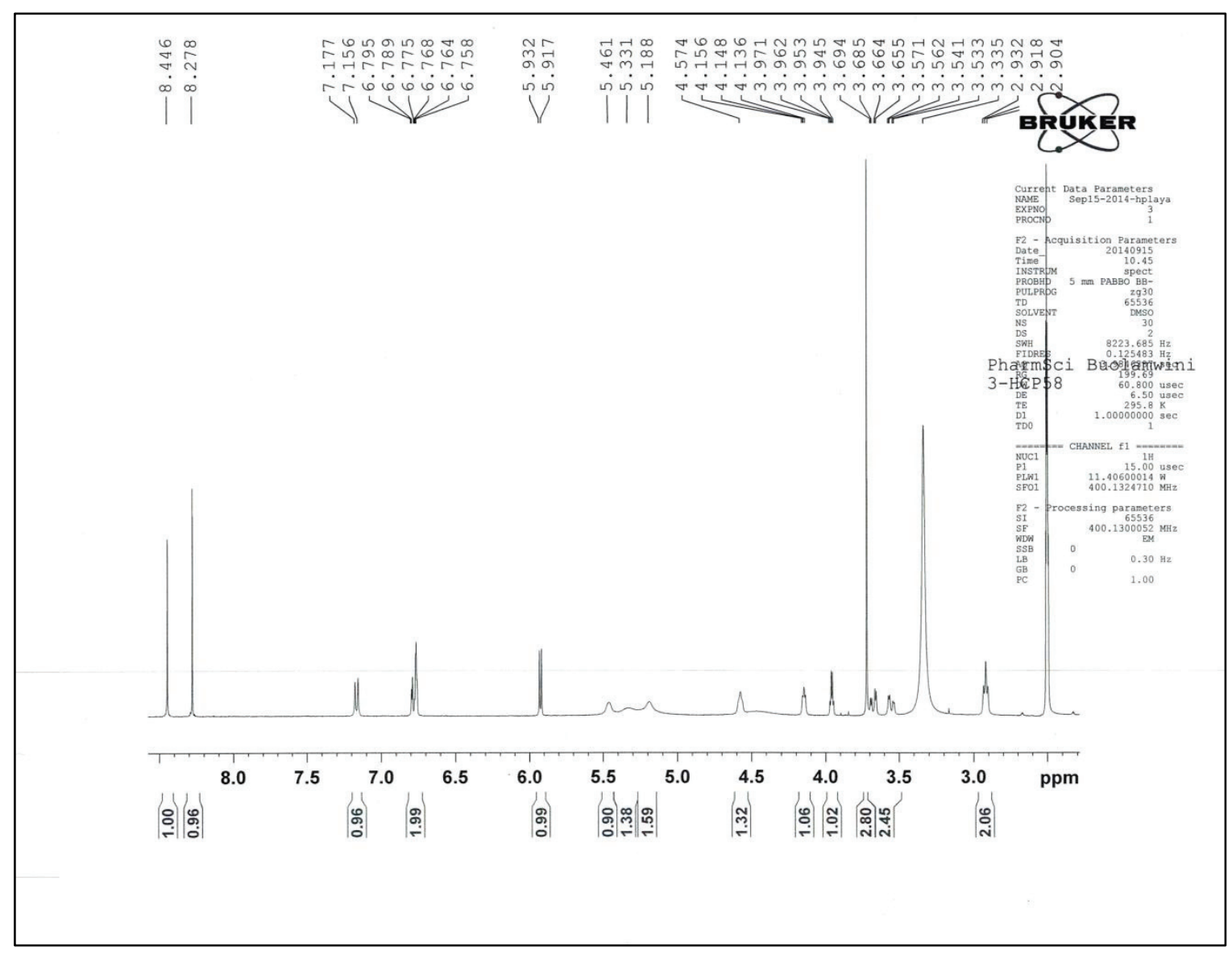

Figure A-40. HCPN58 proton NMR 


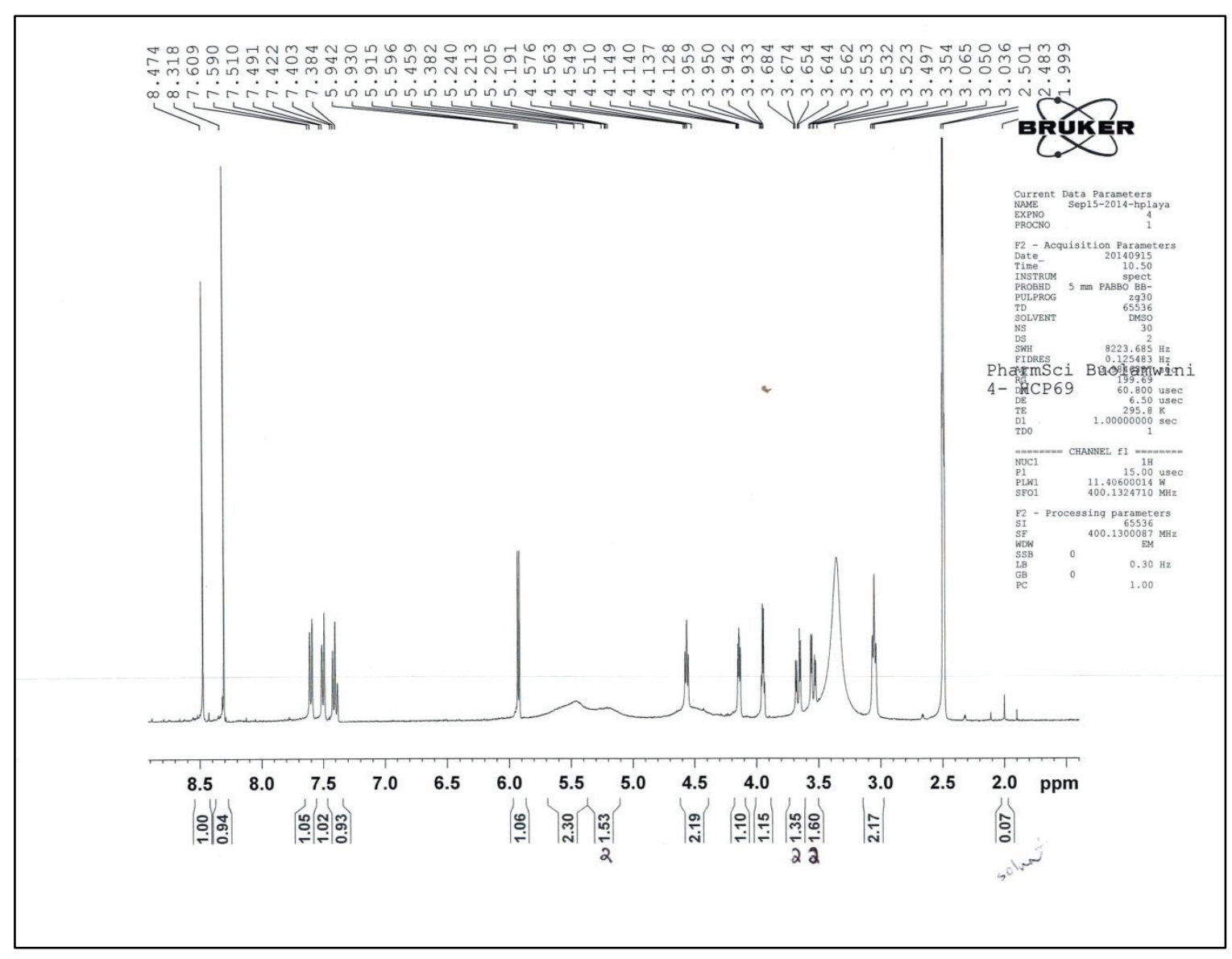

Figure A-41. HCPN69 proton NMR 


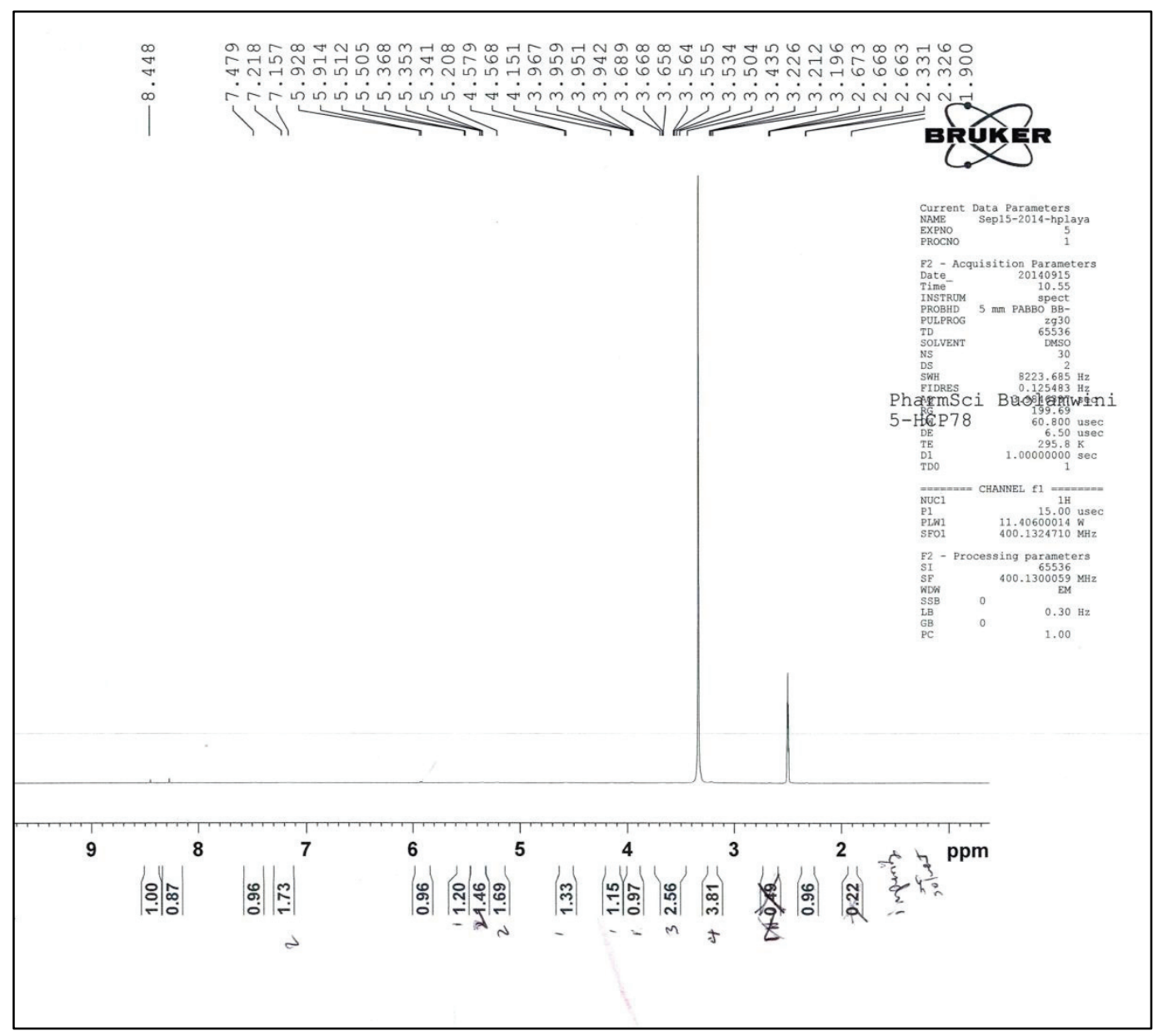

Figure A-42. HCPN78 proton NMR 


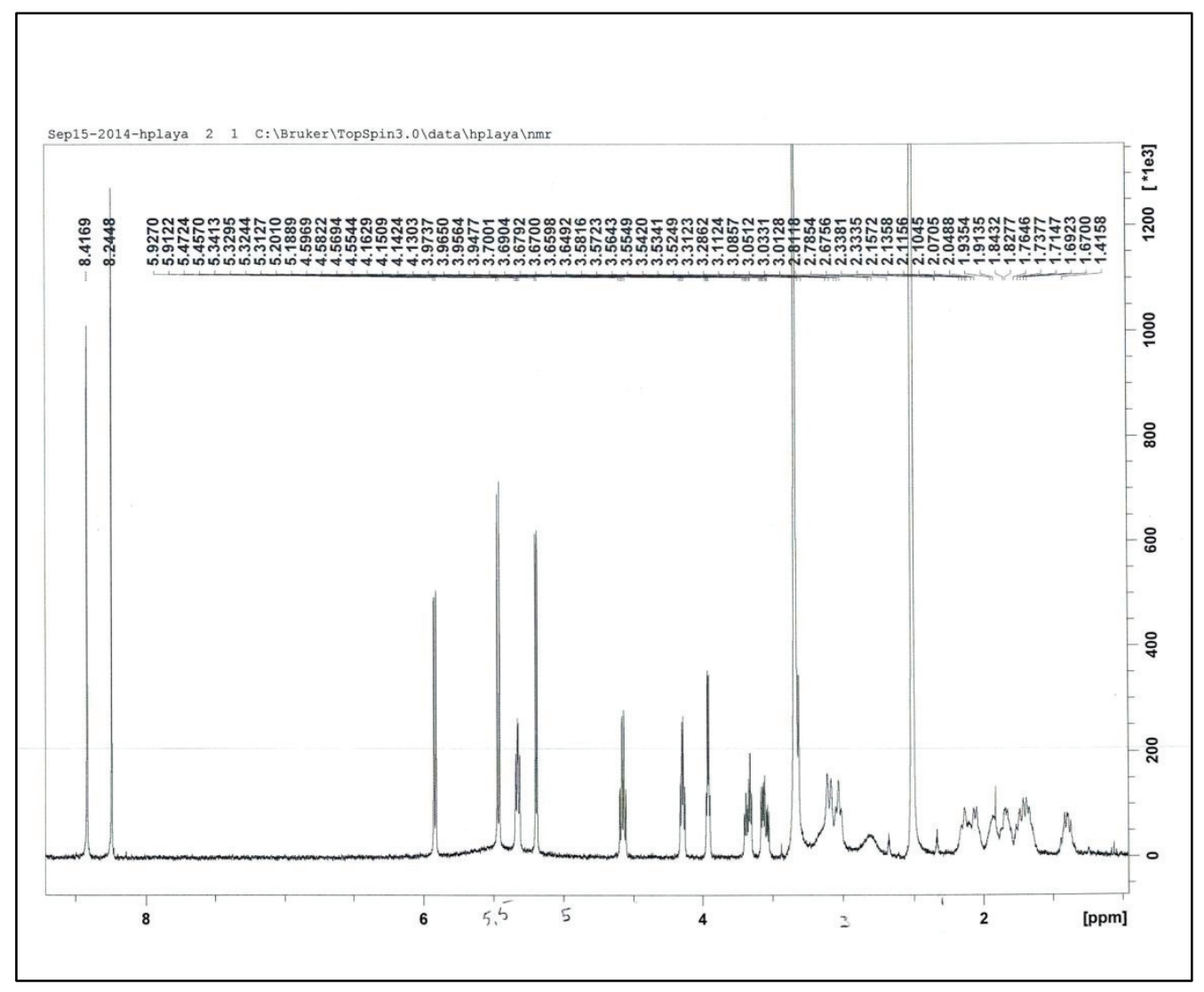

Figure A-43. Expanded HCPN78 proton NMR 


\section{Pyridone Analogues}

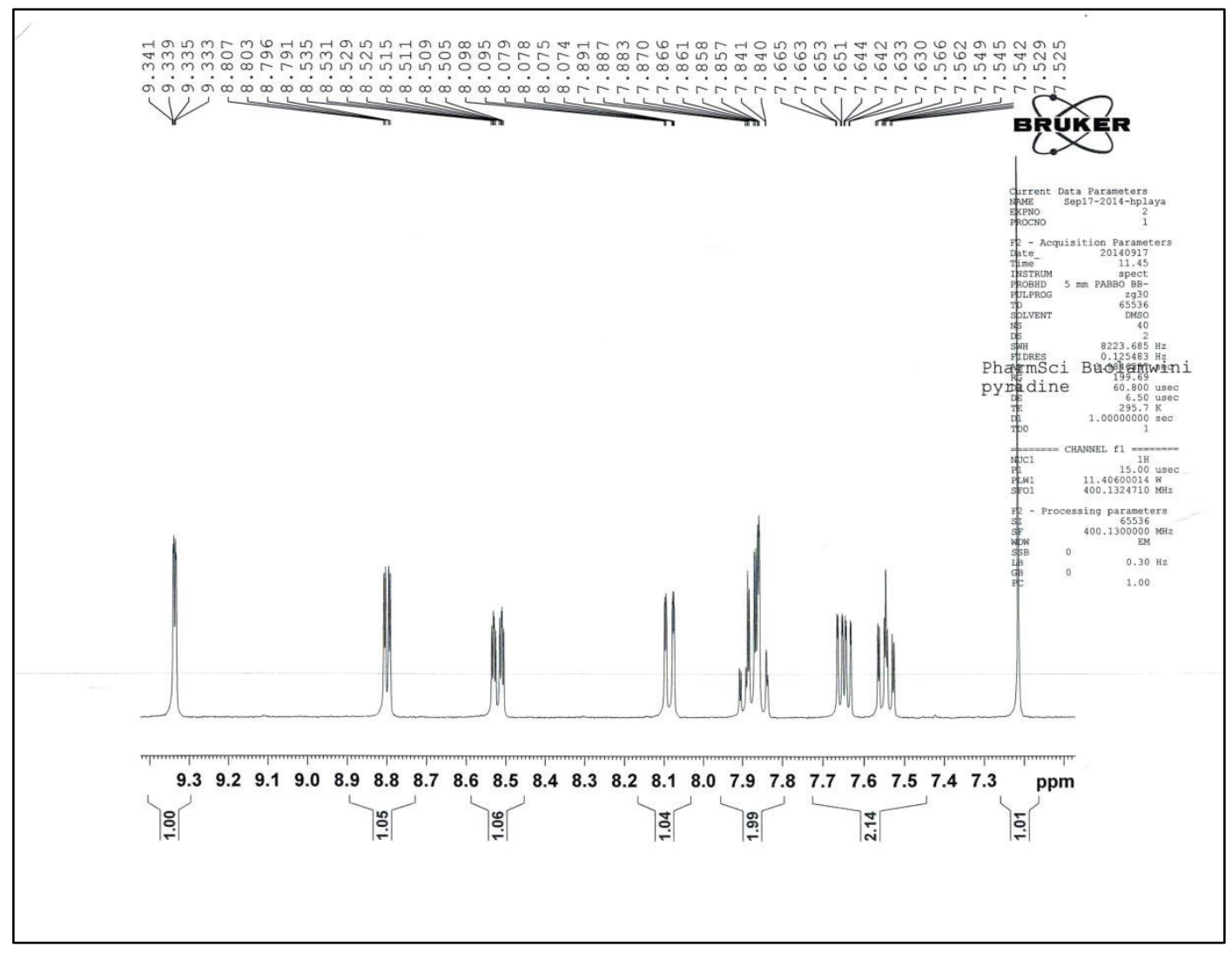

Figure A-44. Pyr2 proton NMR 


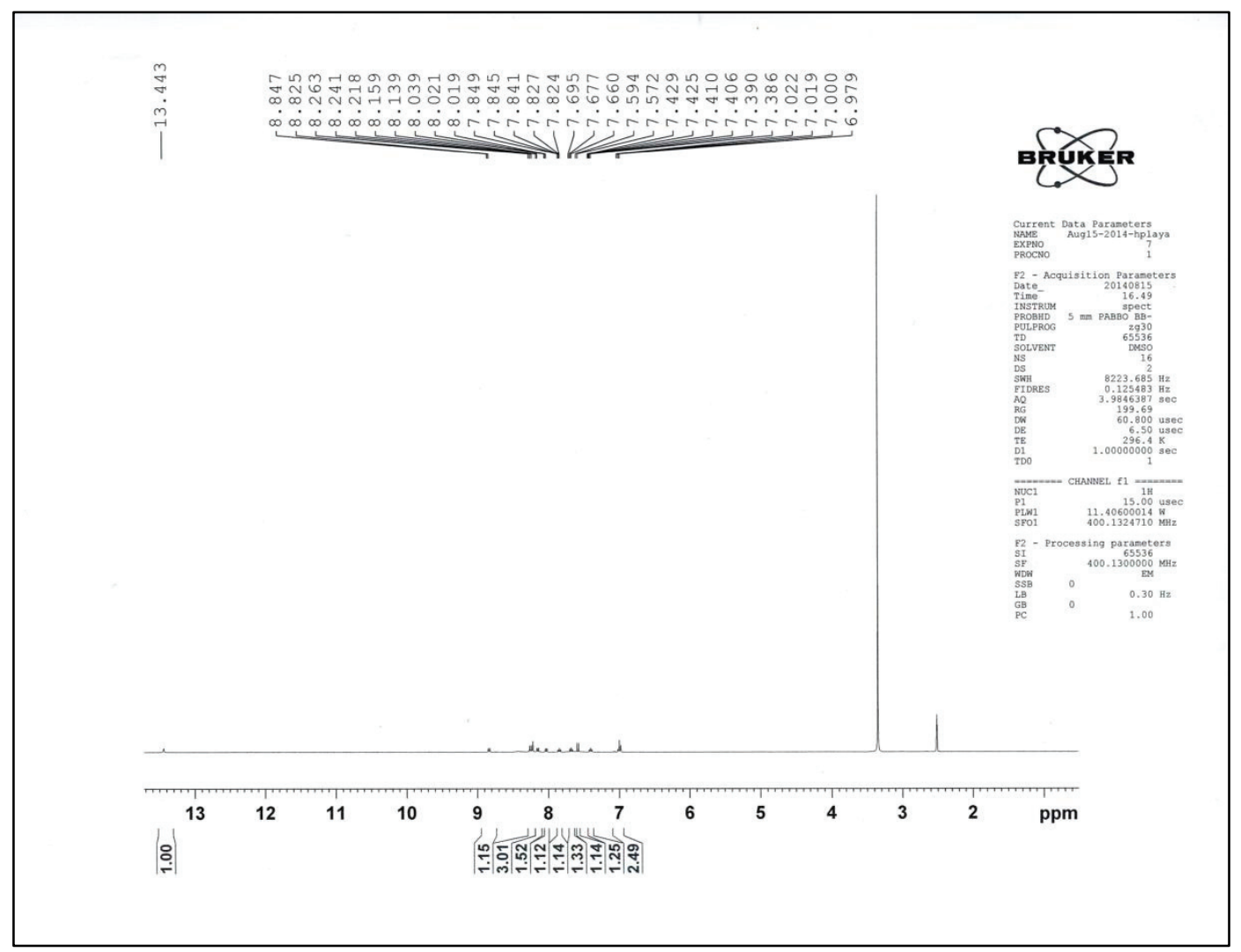

Figure A-45. Pyr3 proton NMR 


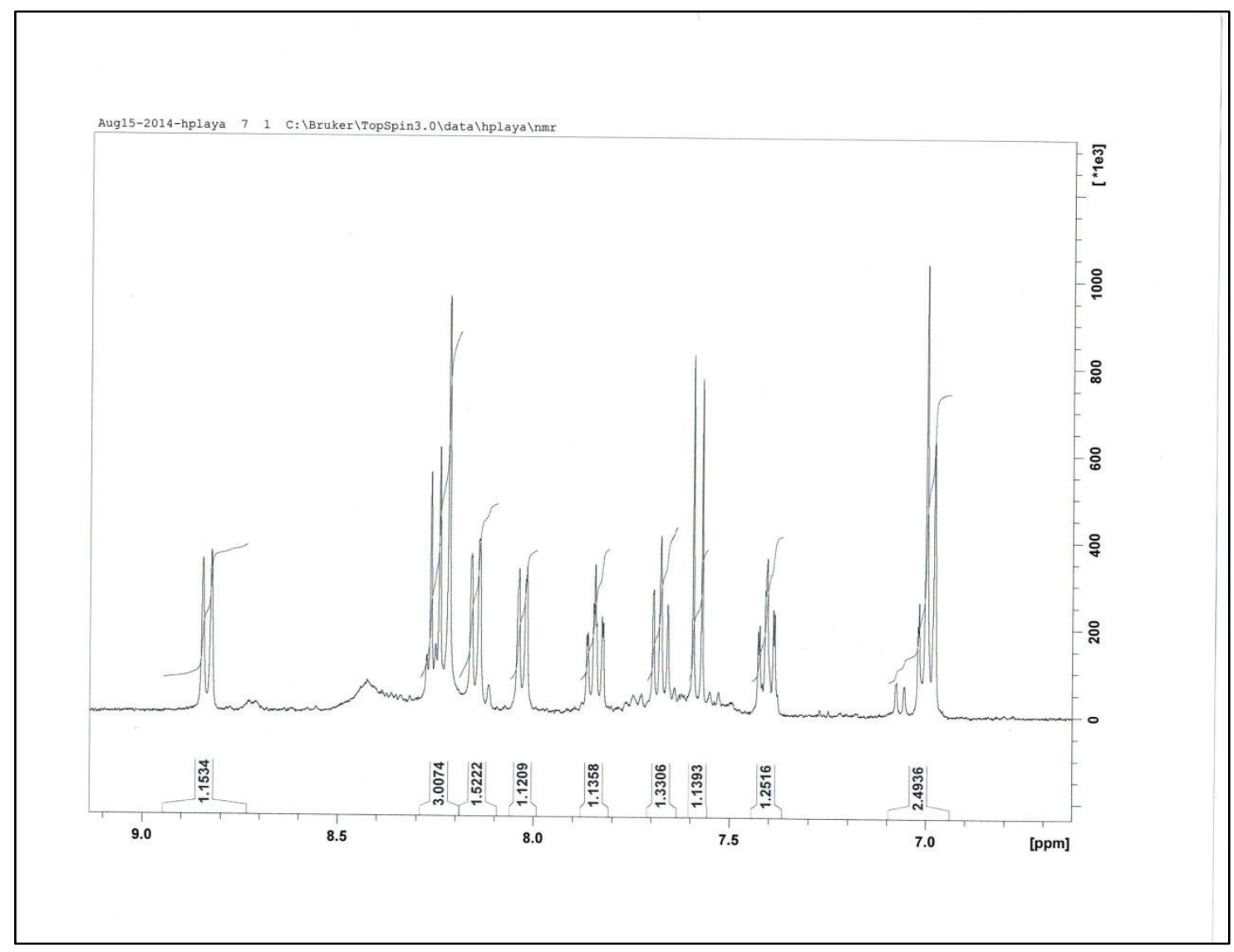

Figure A-46. Expanded Pyr3 proton NMR 


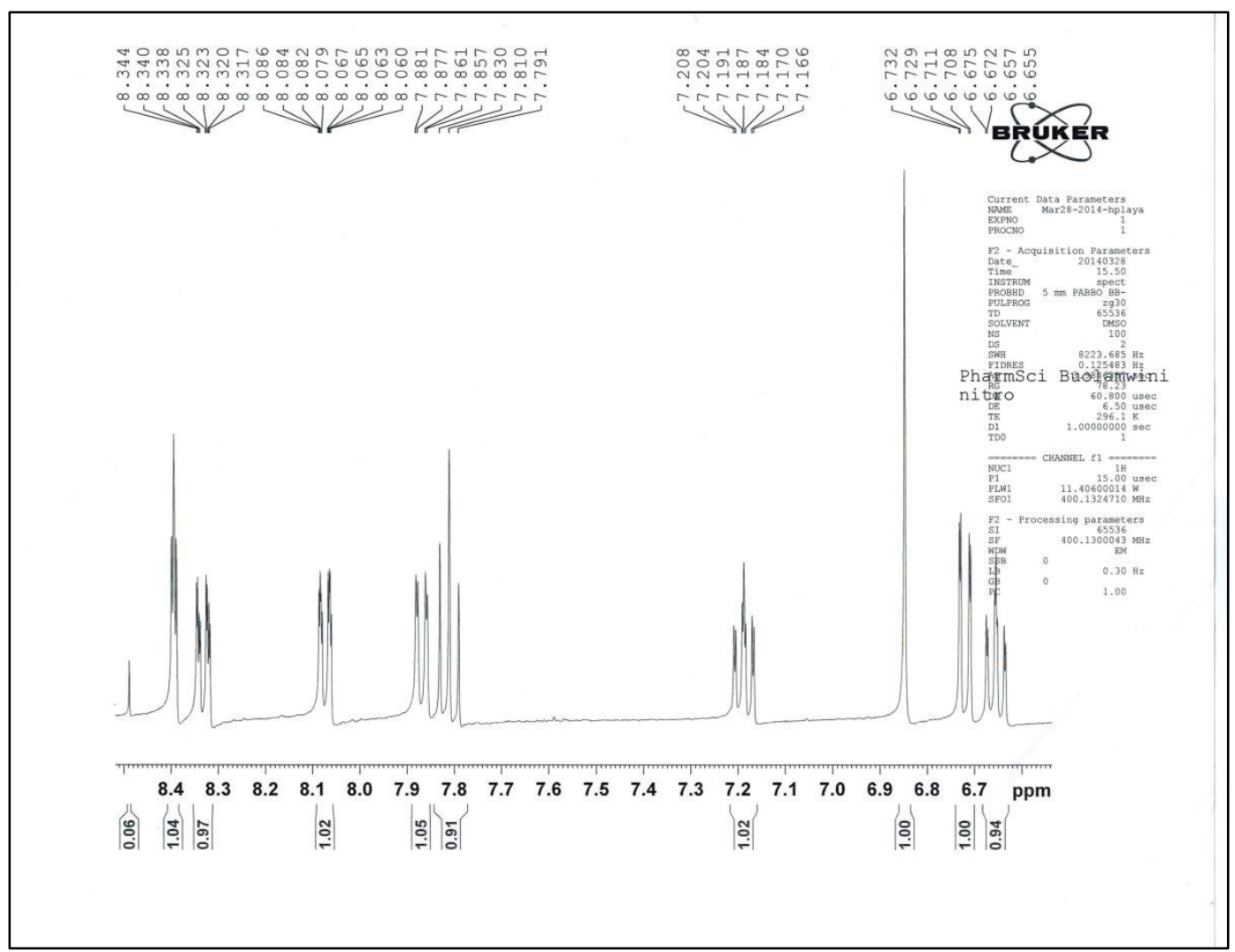

Figure A-47. Pyr4 proton NMR 


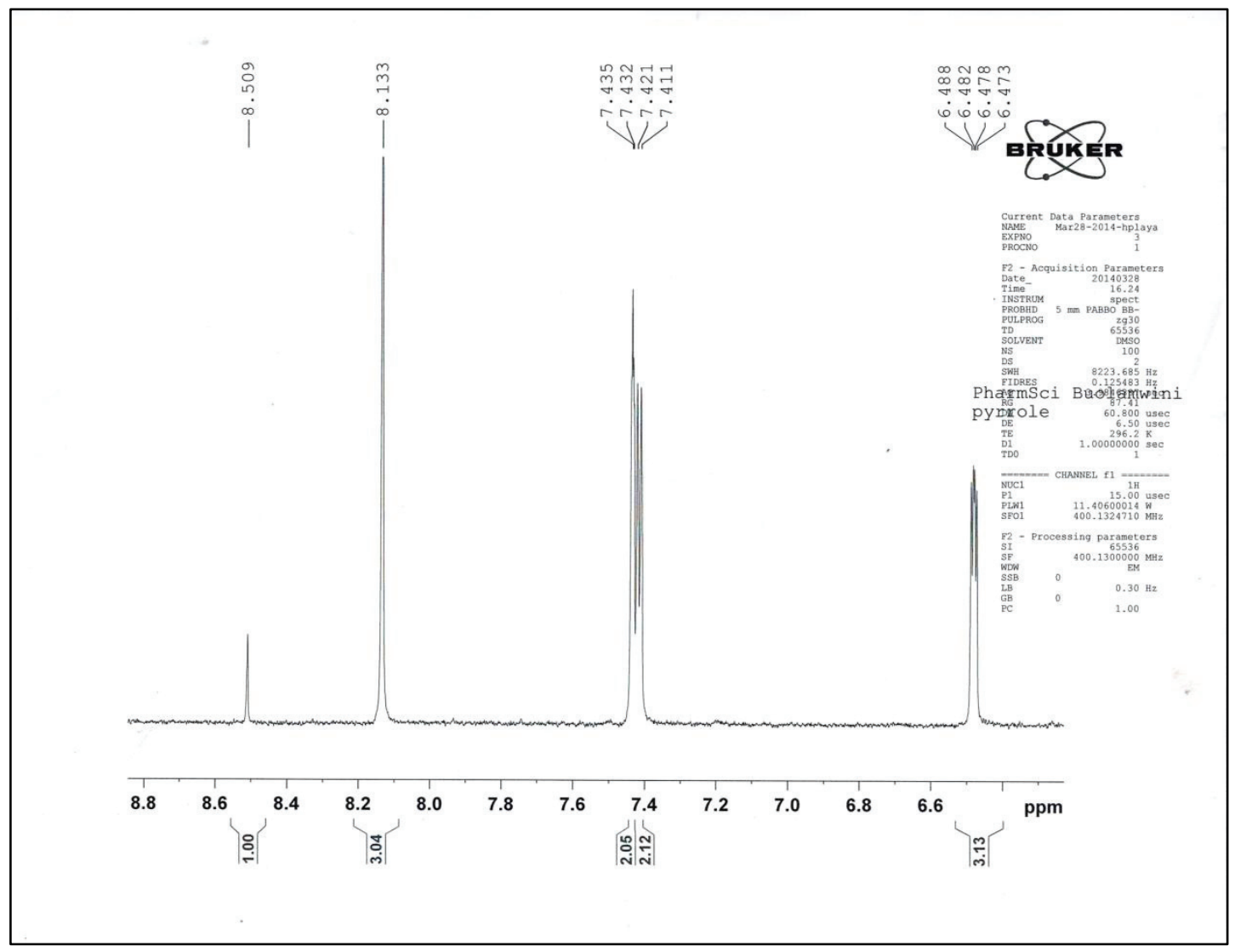

Figure A-48. Pyr6 proton NMR 


\section{VITA}

Born in 1979 as Hilaire Colleen Smith, Hilaire Playa discovered science at a young age. Once in high school, she participated in Science Olympiad, and later the University Interscholastic League science competition, reaching regionals. She was inducted into $\mathrm{Mu}$ Alpha Theta, a math honor society, her senior year as well as the National Honor Society. She graduated magna cum laude, $17^{\text {th }}$ in her class at Deer Park High School in Texas. She earned a chemistry scholarship to Christian Brother's University and completed her B.S. degree in Chemistry with a minor in biology in 2001. After graduating she worked as a research technician in a molecular biology laboratory studying the role of adenosine receptors under hypoxic conditions. Upon acceptance into graduate school, Hilaire resigned and began graduate studies, but withdrew for family reasons. In 2009 she returned to graduate school and completed her studies, culminating in this dissertation. She is a member of the American Chemical Society and the American Association of Pharmaceutical Sciences and was inducted into Rho Chi, an honors society for pharmacists and the pharmaceutical sciences. 